\title{
Palladium-Catalyzed Benzylic C-H Arylation of Azaarylmethylamines
}

\author{
Byeong-Seon Kim, ${ }^{\mathrm{a} \dagger}$ Jacqueline Jiménez, ${ }^{\mathrm{a}, \mathrm{b} \dagger}{ }^{\text {Feng Gao },{ }^{\mathrm{a}, \mathrm{c}} \text { Patrick J. Walsh }}{ }^{\mathrm{a}} *$
}

${ }^{\text {a }}$ Roy and Diana Vagelos Laboratories, Penn/Merck Laboratory for High-Throughput Experimentation, Department of Chemistry, University of Pennsylvania, 231 South 34th Street, Philadelphia, Pennsylvania 19104-6323, United States.

${ }^{\mathrm{b}}$ Facultad de Ciencias Químicas, Benemérita Universidad Autónoma de Puebla, Puebla 72570, Mexico

${ }^{\mathrm{c}}$ Department of Medicinal Plants, Agronomy College, Sichuan Agricultural University, 211, Huimin Rd, Wenjiang Region, Chengdu 611130, PR China

These authors contributed equally.

pwalsh@sas.upenn.edu

Table of Contents

Page

1. General Methods S2

2. Procedures and Characterization of Azaarylmethylamines.

3. Palladium-Catalyzed C $\left(s p^{3}\right)$-H Arylation of Azaarylmethylamines

4. References

S30

5. ${ }^{1} \mathrm{H}$ and ${ }^{13} \mathrm{C}$ NMR Spectra

S31 
1. General Methods. All reactions were conducted under an inert atmosphere of dry nitrogen. Anhydrous 1,4-dioxane and cyclopentyl methyl ether (CPME) were purchased from Sigma-Aldrich and used without further purification. Dimethoxyethane (DME) and tetrahydrofuran (THF) were dried through activated alumina columns under nitrogen. Unless otherwise stated, Silica gel (Silicaflash, P60, 40-63 $\mu \mathrm{m}$, Silicycle) was used for air-flashed chromatography. Other solvents were commercially available and used as received without further purification. Bases $\left[\mathrm{LiN}\left(\mathrm{SiMe}_{3}\right)_{2}, \mathrm{NaN}\left(\mathrm{SiMe}_{3}\right)_{2}\right.$, $\left.\mathrm{KN}\left(\mathrm{SiMe}_{3}\right)_{2}, \mathrm{LiO} t \mathrm{Bu}, \mathrm{NaO} t \mathrm{Bu}, \mathrm{KO} t \mathrm{Bu}\right]$ were purchased from Sigma-Aldrich and used without further purification. Chemicals were purchased from Sigma-Aldrich, Acros, Fisher Scientific or Matrix Scientific and solvents were obtained from Fisher Scientific. Thin-layer chromatography was performed on Whatman precoated silica gel 60 F-254 plates and visualized by ultraviolet light. Flash chromatography was performed with Silica gel (Silicaflash, P60, 40-63 $\mu \mathrm{m}$, Silicycle). NMR spectra were obtained using a Brüker $500 \mathrm{MHz}$ Fourier-transform NMR spectrometer at the University of

Pennsylvania NMR facility. ${ }^{1} \mathrm{H}$ and ${ }^{13} \mathrm{C}$ chemical shifts in parts per million $(\delta)$ were referenced to internal tetramethylsilane (TMS). The infrared spectra were obtained with $\mathrm{KBr}$ plates using a PerkinElmer Spectrum 1600 Series spectrometer. High-resolution mass spectrometry (HRMS) data were obtained on a Waters LC-TOF mass spectrometer (model LCT-XE Premier) using chemical ionization (CI) or electrospray ionization (ESI) in positive or negative mode, depending on the analyte. Melting points were determined on a Unimelt Thomas-Hoover melting point apparatus and are uncorrected. 2(Chloromethyl)pyridine hydrochloride (98\%) and 4-(Chloromethyl)pyridine hydrochloride (98\%) were purchased from CHEM-IMPEX INT'L INC. and used as received.

\section{Preparation of Azaarylmethylamines.}

General Procedure A: In a $50 \mathrm{~mL}$ two-necked flask was added the 2-(chloromethyl)pyridine hydrochloride (3.28 g, $20 \mathrm{mmol})$, sodium iodide (300 mg, $2.0 \mathrm{mmol}), \mathrm{K}_{2} \mathrm{CO}_{3}(13.8 \mathrm{~g}, 100 \mathrm{mmol})$ in 50 
$\mathrm{mL}$ of $\mathrm{CH}_{3} \mathrm{CN}$. The heterogeneous solution was stirred for $5 \mathrm{~min}$ at room temperature. To the reaction mixture was added dropwise amine $(50 \mathrm{mmol})$ and the resulting solution was stirred at room temperature for $12 \mathrm{~h}$ until TLC showed complete consumption of 2-(chloromethyl)pyridine. The reaction mixture was filtered over a pad of celite and the pad was rinsed with additional ethyl acetate (30 $\mathrm{mL}$ ), and the solution was concentrated in vacuo. The crude product was further purified by flash column chromatography on silica gel.

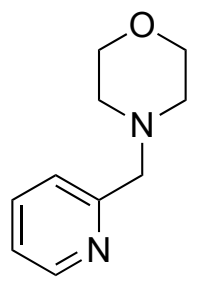

4-(Pyridin-2-ylmethyl)morpholine (1a). The product was prepared by General Procedure A using 2-(chloromethyl)pyridine hydrochloride (3.28 g, $20.0 \mathrm{mmol})$, sodium iodide (300 $\mathrm{mg}, 2.0 \mathrm{mmol}), \mathrm{K}_{2} \mathrm{CO}_{3}(13.8 \mathrm{~g}, 100 \mathrm{mmol})$ and morpholine (4.2 mL, $\left.50.0 \mathrm{mmol}\right)$. The crude product was purified by flash column chromatography on silica gel (only EtOAc) to afford the product $1 \mathrm{a}$ as a brown oil $(3.21 \mathrm{~g}, 90 \%$ yield $) .{ }^{1} \mathrm{H}$ NMR $\left(500 \mathrm{MHz}, \mathrm{CDCl}_{3}\right): \delta 8.56(\mathrm{ddd}, J=4.9,2.0$, 1.0, 1H), $7.64(\mathrm{td}, J=7.6,1.8 \mathrm{~Hz}, 1 \mathrm{H}), 7.39(\operatorname{app~d}, J=7.8 \mathrm{~Hz}, 1 \mathrm{H}), 7.17-7.14(\mathrm{~m}, 1 \mathrm{H}), 3.73(\mathrm{t}, J=4.7$ $\mathrm{Hz}, 4 \mathrm{H}), 3.65(\mathrm{~s}, 2 \mathrm{H}), 2.50(\mathrm{t}, J=4.7 \mathrm{~Hz}, 4 \mathrm{H}) \mathrm{ppm} ;{ }^{13} \mathrm{C}\left\{{ }^{1} \mathrm{H}\right\} \mathrm{NMR}\left(125 \mathrm{MHz}, \mathrm{CDCl}_{3}\right) \delta 158.3,149.4$ 136.5, 123.4, 122.2, 67.1, 65.1, 53.9 ppm; IR (thin film): 3036, 2958, 2855, 2812, 1674, 1590, 1571, $1475,1455,1434,1351,1333,1303,1286,1263,1207,1116,1070,1035,1048,1010 \mathrm{~cm}^{-1} ; \mathrm{HRMS} \mathrm{m} / \mathrm{z}$ 179.1189 [(M+H) ${ }^{+}$; calcd for $\mathrm{C}_{10} \mathrm{H}_{15} \mathrm{~N}_{2} \mathrm{O}$ : 179.1184]. The NMR spectral data match the previously published data. ${ }^{1}$

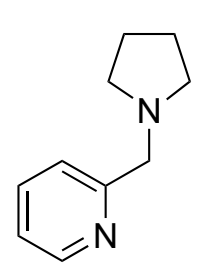

2-(Pyrrolidin-1-ylmethyl)pyridine (1b). The product was prepared by General Procedure A using 2-(chloromethyl)pyridine hydrochloride (3.28 g, $20.0 \mathrm{mmol})$, sodium iodide (300 $\mathrm{mg}, 2.0 \mathrm{mmol}), \mathrm{K}_{2} \mathrm{CO}_{3}(13.8 \mathrm{~g}, 100.0 \mathrm{mmol})$ and pyrrolidine $(4.1 \mathrm{~mL}, 50.0 \mathrm{mmol})$. The crude product was purified by flash column chromatography on silica gel (hexanes:EtOAc $=50: 50$ ) to give the product $\mathbf{1 b}$ as an orange oil $\left(1.81 \mathrm{~g}, 56 \%\right.$ yield). ${ }^{1} \mathrm{H}$ NMR $\left(500 \mathrm{MHz}, \mathrm{CDCl}_{3}\right): \delta 8.56(\mathrm{ddd}, J=$ 
5.0, 2.0, 1.0, 1H), $7.64(\mathrm{td}, J=7.8,2.0 \mathrm{~Hz}, 1 \mathrm{H}), 7.39(\operatorname{app~d}, J=8.0 \mathrm{~Hz}, 1 \mathrm{H}), 7.14(\mathrm{ddd}, J=7.8,5.0,1.0$ $\mathrm{Hz}, 1 \mathrm{H}), 3.78(\mathrm{~s}, 2 \mathrm{H}), 2.56(\mathrm{t}, J=4.7 \mathrm{~Hz}, 4 \mathrm{H}), 1.81(\mathrm{t}, J=4.7 \mathrm{~Hz}, 4 \mathrm{H}) \mathrm{ppm} ;{ }^{13} \mathrm{C}\left\{{ }^{1} \mathrm{H}\right\} \mathrm{NMR}(125 \mathrm{MHz}$ $\left.\mathrm{CDCl}_{3}\right) \delta 159.5,149.1,136.3,122.9,121.8,62.3,54.3,23.6 \mathrm{ppm} ;$ IR (thin film): 2974, 1609, 1588, 1433, 1337, 1104, $699 \mathrm{~cm}^{-1}$; HRMS m/z $163.1230\left[(\mathrm{M}+\mathrm{H})^{+}\right.$; calcd for $\mathrm{C}_{10} \mathrm{H}_{15} \mathrm{~N}_{2}$ : 163.1235]. The NMR spectral data match the previously published data., ${ }^{2,3}$

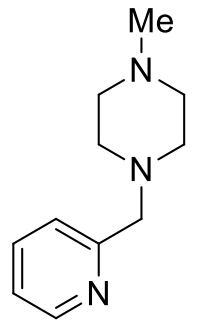

1-Methyl-4-(pyridin-2-ylmethyl)piperazine 1c. The product was prepared by General Procedure A using 2-(chloromethyl)pyridine hydrochloride (3.28 g, $20.0 \mathrm{mmol})$, sodium iodide (600 mg, $4.0 \mathrm{mmol})$, potassium carbonate $(13.8 \mathrm{~g}, 100.0 \mathrm{mmol})$ and 1 methylpiperazine $(5.5 \mathrm{~mL}, 50.0 \mathrm{mmol})$. The crude product was purified by flash column chromatography on silica gel (EtOAc) to give the product $1 \mathrm{c}$ as a yellow oil $\left(2.98 \mathrm{~g}, 78 \%\right.$ yield). ${ }^{1} \mathrm{H}$ $\operatorname{NMR}\left(500 \mathrm{MHz}, \mathrm{CDCl}_{3}\right): \delta 8.56(\mathrm{ddd}, J=5.0,2.0,1.0,1 \mathrm{H}), 7.64(\mathrm{td}, J=8.0,2.0 \mathrm{~Hz}, 1 \mathrm{H}), 7.39$ (app d, $J=8.0 \mathrm{~Hz}, 1 \mathrm{H}), 7.15(\mathrm{ddd}, J=7.5,5.0,1.0 \mathrm{~Hz}, 1 \mathrm{H}), 3.67(\mathrm{~s}, 2 \mathrm{H}), 2.90-2.20(\mathrm{~m}, 8 \mathrm{H}), 2.29(\mathrm{~s}, 3 \mathrm{H})$ ppm; ${ }^{13} \mathrm{C}\left\{{ }^{1} \mathrm{H}\right\}$ NMR $\left(125 \mathrm{MHz}, \mathrm{CDCl}_{3}\right) \delta 158.6,149.3,136.3,123.2,122.0,64.6,55.1,53.3,46.1$ ppm; IR (thin film): 2944, 2810, 1656, 1595, 1571, 1459, 1437, 1353, 1286, 1163, 1150, 1136, 1051, 1009 $\mathrm{cm}^{-1}$; HRMS m/z 192.1484 [(M+H) ${ }^{+}$; calcd for $\left.\mathrm{C}_{11} \mathrm{H}_{18} \mathrm{~N}_{3}: 192.1501\right]$ (only choice). The NMR spectral data match the previously published data. ${ }^{4}$

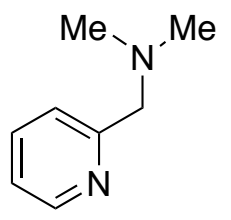

$N, N$-Dimethyl-1-(pyridin-2-yl)methanamine (1d). The product was prepared by General Procedure A using 2-(chloromethyl)pyridine hydrochloride (3.28 g, $20.0 \mathrm{mmol})$, sodium iodide $(300 \mathrm{mg}, 2.0 \mathrm{mmol}), \mathrm{K}_{2} \mathrm{CO}_{3}(13.8 \mathrm{~g}, 100.0 \mathrm{mmol})$ and dimethylamine solution $5.6 \mathrm{M}$ in ethanol $(8.9 \mathrm{~mL}, 50.0 \mathrm{mmol})$. The crude product was purified by flash column chromatography on silica gel (hexanes:EtOAc $=30: 70)$ to give the product $\mathbf{1 d}$ as a yellow oil $(1.63 \mathrm{~g}$, 
$60 \%$ yield). ${ }^{1} \mathrm{H}$ NMR $\left(500 \mathrm{MHz}, \mathrm{CDCl}_{3}\right): \delta 8.56(\mathrm{ddd}, J=5.0,2.0,1.0,1 \mathrm{H}), 7.64(\mathrm{td}, J=8.0,2.0 \mathrm{~Hz}$ 1H), $7.39(\operatorname{app~d}, J=8.0 \mathrm{~Hz}, 1 \mathrm{H}), 7.16(\mathrm{ddd}, J=7.5,5.0,1.0 \mathrm{~Hz}, 1 \mathrm{H}), 3.59(\mathrm{~s}, 2 \mathrm{H}), 2.30$ (s, 6H) ppm; ${ }^{13} \mathrm{C}\left\{{ }^{1} \mathrm{H}\right\} \operatorname{NMR}\left(125 \mathrm{MHz}, \mathrm{CDCl}_{3}\right) \delta 159.4,149.4,136.5,123.2,122.1,66.0,45.8 \mathrm{ppm}$; IR (thin film): $3067,2975,2945,2860,2822,2776,1591,1570,1474,1456,1436,1361,1258,1173,1152,1097,1040$ $\mathrm{cm}^{-1}$; HRMS m/z $137.1078\left[(\mathrm{M}+\mathrm{H})^{+}\right.$; calcd for $\left.\mathrm{C}_{8} \mathrm{H}_{13} \mathrm{~N}_{2}: 137.1079\right]$. The NMR spectral data match the previously published data. ${ }^{5}$

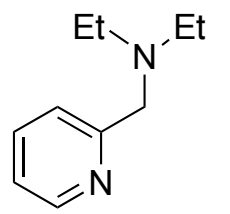

$N$-Ethyl- $N$-(pyridin-2-ylmethyl)ethanamine 1e. The product was prepared by General Procedure A using 2-(chloromethyl)pyridine hydrochloride (3.28 g, 20.0 mmol), sodium iodide $(599.6 \mathrm{mg}, 4.0 \mathrm{mmol})$, potassium carbonate $(13.8 \mathrm{~g}, 100.0 \mathrm{mmol})$ and diethylamine (3.66 g, $5.2 \mathrm{~mL}, 50.0 \mathrm{mmol})$. The crude product was purified by flash column chromatography on silica gel (hexanes:EtOAc $=50: 50)$ to give the product 1e as an orange oil $(2.89 \mathrm{~g}$, $88 \%$ yield). ${ }^{1} \mathrm{H}$ NMR (500 MHz, $\left.\mathrm{CDCl}_{3}\right): \delta 8.53(\mathrm{ddd}, J=5.0,2.0,1.0 \mathrm{~Hz}, 1 \mathrm{H}), 7.63(\mathrm{td}, J=8.0,2.0 \mathrm{~Hz}$, 1H), $7.47(\operatorname{app~d}, J=8.0 \mathrm{~Hz}, 1 \mathrm{H}), 7.12(\mathrm{ddd}, J=7.5,5.0,1.0 \mathrm{~Hz}, 1 \mathrm{H}), 3.72(\mathrm{~s}, 2 \mathrm{H}), 2.58(\mathrm{q}, J=7.2 \mathrm{~Hz}$ 4H), $1.05(\mathrm{t}, J=7.2 \mathrm{~Hz}, 6 \mathrm{H}) \mathrm{ppm} ;{ }^{13} \mathrm{C}\left\{{ }^{1} \mathrm{H}\right\}$ NMR $\left(125 \mathrm{MHz}, \mathrm{CDCl}_{3}\right) \delta 160.9,149.1,136.3,122.0$, 121.7, 59.8, 47.5, 12.0 ppm; The NMR spectral data match the previously published data. ${ }^{6}$

$N$-Benzyl- $N$-methyl-1-(pyridin-2-yl)methanamine 1f. The product was prepared by
Sodium iodide $(300 \mathrm{mg}, \quad 2.0 \mathrm{mmol}), \mathrm{K}_{2} \mathrm{CO}_{3}(13.8 \mathrm{~g}, \quad 100.0 \mathrm{mmol})$ and benzylmethylamine $(6.06 \mathrm{~g}, 6.5 \mathrm{~mL}, 50.0 \mathrm{mmol})$. The crude product was purified by flash column chromatography on silica gel (hexanes:EtOAc $=50: 50)$ to give the product $\mathbf{1 f}$ as a yellow oil $(3.27 \mathrm{~g}$, 77\% yield). ${ }^{1} \mathrm{H}$ NMR (500 MHz, $\left.\mathrm{CDCl}_{3}\right) \delta 8.54(\mathrm{dt}, J=4.8,0.8 \mathrm{~Hz}, 1 \mathrm{H}), 7.65(\mathrm{td}, J=7.7,1.8 \mathrm{~Hz}, 1 \mathrm{H})$, 
$7.51(\mathrm{~d}, J=7.9 \mathrm{~Hz}, 1 \mathrm{H}), 7.37(\mathrm{~d}, J=7.0 \mathrm{~Hz}, 2 \mathrm{H}), 7.32(\mathrm{t}, J=7.5 \mathrm{~Hz}, 2 \mathrm{H}), 7.26-7.23(\mathrm{~m}, 1 \mathrm{H}), 7.15-$

$7.13(\mathrm{~m}, 1 \mathrm{H}), 3.70(\mathrm{~s}, 2 \mathrm{H}), 3.59(\mathrm{~s}, 2 \mathrm{H}), 2.25(\mathrm{~s}, 3 \mathrm{H}) ;{ }^{13} \mathrm{C} \mathrm{NMR}\left(125 \mathrm{MHz}, \mathrm{CDCl}_{3}\right) \delta 160.0,149.2$ 139.3, 136.6, 129.1, 128.4, 127.2, 123.1, 122.1, 63.7, 62.3, 42.7; IR (neat) 3061, 3028, 2978, 2944, 2924, 2838, 2790, 1590, 1570, 1495, 1474, 1454, 1433, 1364, $1244 \mathrm{~cm}^{-1} ; \mathrm{HRMS} \mathrm{m} / \mathrm{z} 213.1389$ $\left[(\mathrm{M}+\mathrm{H})^{+}\right.$; calcd for $\left.\mathrm{C}_{14} \mathrm{H}_{18} \mathrm{~N}_{2}: 213.1392\right]$. The NMR spectral data match the previously published data. ${ }^{7}$

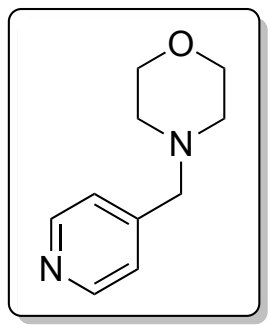

4-(Pyridin-4-ylmethyl)morpholine (1g). The product was prepared by General Procedure A using 4-(chloromethyl)pyridine hydrochloride (3.28 g, $20.0 \mathrm{mmol})$, sodium iodide (599.6 mg, $4.0 \mathrm{mmol})$, potassium carbonate $(13.8 \mathrm{~g}, 100.0 \mathrm{mmol})$ and morpholine $(4.3 \mathrm{~mL}, 50.0 \mathrm{mmol})$. The crude product was purified by flash column chromatography on silica gel (EtOAc) to afford the product $1 \mathrm{~g}$ as a brown oil $\left(3.28 \mathrm{~g}, 92 \%\right.$ yield). ${ }^{1} \mathrm{H}$ NMR (500 MHz, $\left.\mathrm{CDCl}_{3}\right): \delta 8.54(\operatorname{app~d}, J=4.5 \mathrm{~Hz}, 2 \mathrm{H}), 7.28(\operatorname{app} \mathrm{d}, J=4.5 \mathrm{~Hz}, 2 \mathrm{H}), 3.72(\mathrm{t}, J=4.6$ $\mathrm{Hz}, 4 \mathrm{H}), 3.50(\mathrm{~s}, 2 \mathrm{H}), 2.45(\mathrm{t}, J=4.5 \mathrm{~Hz}, 4 \mathrm{H}) \mathrm{ppm} ;{ }^{13} \mathrm{C}\left\{{ }^{1} \mathrm{H}\right\} \mathrm{NMR}\left(125 \mathrm{MHz}, \mathrm{CDCl}_{3}\right) \delta 149.9,147.3$, 123.9, 67.0, 62.0, $53.8 \mathrm{ppm}$; HRMS m/z $179.1183\left[(\mathrm{M}+\mathrm{H})^{+}\right.$; calcd for $\left.\mathrm{C}_{10} \mathrm{H}_{15} \mathrm{~N}_{2} \mathrm{O}: 179.1184\right]$. The NMR spectral data match the previously published data. ${ }^{1}$

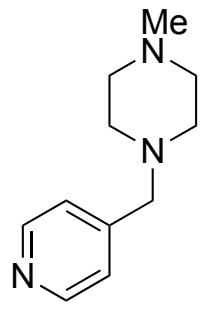

1-Methyl-4-(pyridin-4-ylmethyl)piperazine 1h. The product was prepared by General Procedure A using 4-(chloromethyl)pyridine hydrochloride (3.28 g, $20.0 \mathrm{mmol})$, sodium iodide (599.6 mg, $4.0 \mathrm{mmol})$, potassium carbonate $(13.8 \mathrm{~g}, 100.0 \mathrm{mmol})$ and 1 methylpiperazine $(5.0 \mathrm{~g}, 5.5 \mathrm{~mL}, 50.0 \mathrm{mmol})$. The crude product was purified by flash column chromatography on silica gel (only EtOAc) to give the product $\mathbf{1 h}$ as a yellow oil (3.06 $\mathrm{g}, 80 \%$ yield). ${ }^{1} \mathrm{H}$ NMR $\left(500 \mathrm{MHz}, \mathrm{CDCl}_{3}\right) \delta 8.54(\mathrm{dd}, J=4.4,1.6 \mathrm{~Hz}, 2 \mathrm{H}), 7.27-7.26(\mathrm{~m}, 2 \mathrm{H}), 3.50(\mathrm{~s}, 2 \mathrm{H})$, $2.53-2.42(\mathrm{~m}, 8 \mathrm{H}), 2.29(\mathrm{~s}, 3 \mathrm{H}) ;{ }^{13} \mathrm{C} \mathrm{NMR}\left(125 \mathrm{MHz}, \mathrm{CDCl}_{3}\right) \delta 149.8,147.7,123.8,61.7,55.1,53.2$ 
46.1; IR (neat) 2942, 2884, 2808, 1604, 1562, 1458, 1418, 1372, 1356, 1323, 1294, 1284, $1223 \mathrm{~cm}^{-1}$; HRMS m/z $192.1501\left[(\mathrm{M}+\mathrm{H})^{+}\right.$; calcd for $\left.\mathrm{C}_{11} \mathrm{H}_{18} \mathrm{~N}_{3}: 192.1501\right]$.

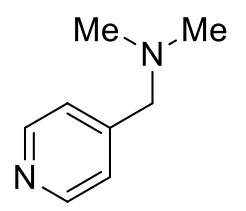

$N, N$-Dimethyl-1-(pyridin-4-yl)methanamine 1i. The product was prepared by General Procedure A using 4-(chloromethyl)pyridine hydrochloride (3.28 g, $20.0 \mathrm{mmol})$, sodium iodide (300 mg, $2.0 \mathrm{mmol}), \mathrm{K}_{2} \mathrm{CO}_{3}(13.8 \mathrm{~g}, 100.0 \mathrm{mmol})$ and dimethylamine solution $5.6 \mathrm{M}$ in ethanol $(8.9 \mathrm{~mL}, 50.0 \mathrm{mmol})$. The crude product was purified by flash column chromatography on silica gel (hexanes:EtOAc $=30: 70)$ to give the product $\mathbf{1} \mathbf{i}$ as a yellow oil $\left(1.83 \mathrm{~g}, 67 \%\right.$ yield). ${ }^{1} \mathrm{H}$ NMR (500 MHz, $\left.\mathrm{CDCl}_{3}\right) \delta 8.52(\mathrm{~d}, J=5.0 \mathrm{~Hz}, 2 \mathrm{H}), 7.22(\mathrm{~d}, J=5.0 \mathrm{~Hz}, 2 \mathrm{H}), 3.39(\mathrm{~s}, 2 \mathrm{H}), 2.22(\mathrm{~s}, 6 \mathrm{H})$; ${ }^{13} \mathrm{C}$ NMR $\left(125 \mathrm{MHz}, \mathrm{CDCl}_{3}\right) \delta 150.0,148.3,124.0,63.3,45.7$. The ${ }^{1} \mathrm{H}$ NMR spectral data match the previously published data. $^{5}$

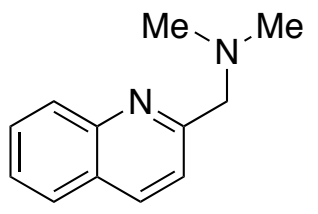

$N, N$-Dimethyl-1-(quinolin-2-yl)methanamines $1 \mathbf{j}$. The product was prepared by General Procedure A using 2-(chloromethyl)quinoline hydrochloride (0.64 g, 3.0 mmol), sodium iodide $(89.9 \mathrm{mg}, 0.6 \mathrm{mmol})$, potassium carbonate $(2.10 \mathrm{~g}, 15.0$ mmol) and dimethylamine solution $5.6 \mathrm{M}$ in ethanol $(1.34 \mathrm{~mL}, 7.5 \mathrm{mmol})$. The crude product was purified by flash column chromatography on silica gel (hexanes:EtOAc $=50: 50$ ) to give the product $\mathbf{1} \mathbf{j}$ as a yellow oil $\left(0.47 \mathrm{~g}, 84 \%\right.$ yield). ${ }^{1} \mathrm{H}$ NMR $\left(500 \mathrm{MHz}, \mathrm{CDCl}_{3}\right) \delta 8.11(\mathrm{dd}, J=13.4,8.5 \mathrm{~Hz}, 2 \mathrm{H}), 7.78$ $(\mathrm{dd}, J=8.1,0.8 \mathrm{~Hz}, 1 \mathrm{H}), 7.69(\mathrm{ddd}, J=8.4,7.0,1.4 \mathrm{~Hz}, 1 \mathrm{H}), 7.58(\mathrm{~d}, J=8.5 \mathrm{~Hz}, 1 \mathrm{H}), 7.50(\mathrm{ddd}, J=$ 8.4, 7.0, $1.4 \mathrm{~Hz}, 1 \mathrm{H}), 3.77$ (s, 2H), $2.34(\mathrm{~s}, 6 \mathrm{H}) ;{ }^{13} \mathrm{C} \mathrm{NMR}\left(125 \mathrm{MHz}, \mathrm{CDCl}_{3}\right) \delta$ 160.2, 147.9, 136.6, 129.5, 129.3, 127.7, 127.6, 126.3, 121.3, 66.8, 46.0; IR (neat) 3060, 2974, 2943, 2858, 2820, 2772, $1615,1601,1561,1504,1455,1425,1376,1362,1311,1259,1225 \mathrm{~cm}^{-1}$; HRMS m/z 187.1236 $\left[(\mathrm{M}+\mathrm{H})^{+}\right.$; calcd for $\mathrm{C}_{12} \mathrm{H}_{15} \mathrm{~N}_{2}: 187.1235$. 


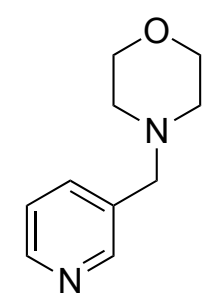

4-(Pyridin-3-ylmethyl)morpholine (1k). The product was prepared by General Procedure A using 3-(chloromethyl)pyridine hydrochloride (3.28 g, $20.0 \mathrm{mmol})$, sodium iodide $(599.6 \mathrm{mg}, 4.0 \mathrm{mmol})$, potassium carbonate $(13.8 \mathrm{~g}, 100.0 \mathrm{mmol})$ and morpholine $(4.3 \mathrm{~mL}, 50.0 \mathrm{mmol})$. The crude product was purified by flash column chromatography on silica gel (EtOAc) to afford the product $\mathbf{1 k}$ as a brown oil (3.03 $\mathrm{g}, 85 \%$ yield). The NMR spectral data match the previously published data. ${ }^{8}$

\section{Palladium-Catalyzed C(sp $\left.{ }^{3}\right)$-H Arylation of Azaarylmethylamines}

Preparation of Pd(OAc) $)_{2}$ /NIXANTPHOS Stock Solution. An oven-dried $20 \mathrm{~mL}$ vial with a stir bar under a nitrogen atmosphere was charged with $\mathrm{Pd}(\mathrm{OAc})_{2}(22.5 \mathrm{mg}, 0.1 \mathrm{mmol})$, NIXANTPHOS (82.7 $\mathrm{mg}, 0.15 \mathrm{mmol})$ and 1,4-dioxane $(10 \mathrm{~mL})(1 \mathrm{~mL}$ of the stock solution was used for $0.2 \mathrm{mmol}$ scale). The solution was stirred for 5 mins before use.

General Procedure B: An oven-dried $10 \mathrm{~mL}$ reaction vial equipped with a stir bar in a glove box under a nitrogen atmosphere. $1 \mathrm{~mL}$ of $\mathrm{Pd}(\mathrm{OAc})_{2} / \mathrm{NIXANTPHOS}$ stock solution $\left(5 \mathrm{~mol} \% \mathrm{Pd}(\mathrm{OAc})_{2} / 7.5 \mathrm{~mol} \%\right.$ NIXANTPHOS) was taken up by syringe and added to the reaction vial. LiHMDS (66.9 mg, $0.4 \mathrm{mmol}$, 2 equiv) was added to the vial. The vial was capped, removed from the glove box and the solution was stirred for 5 minutes. The azaarylmethylamine $(0.2 \mathrm{mmol})$ and aryl halide $(0.24 \mathrm{mmol})$ were added to the solution. Note that aryl bromide in solid form was added to the reaction vial prior to $\operatorname{LiN}\left(\mathrm{SiMe}_{3}\right)_{2}$. The reaction mixture was heated to $65{ }^{\circ} \mathrm{C}$ in an oil bath and stirred for 12 hours until TLC showed 
complete consumption of azaarylmethylamines. The sealed vial was cooled to room temperature and 3 drops of water was added. The reaction mixture was diluted with diethyl ether $(3 \mathrm{~mL})$ or ethyl acetate $(3$ $\mathrm{mL})$ and filtered over a short pad of celite. The pad was rinsed with additional diethyl ether $(5 \mathrm{~mL})$ or ethyl acetate $(5 \mathrm{~mL})$, and the solution was concentrated in vacuo to yield a brown oil. The crude material was loaded onto a silica gel column and purified by flash chromatography.

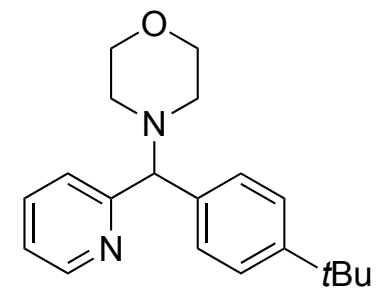

4-((4-(tert-Butyl)phenyl)(pyridin-2-yl)methyl)morpholine (3a). The product was prepared by General Procedure B using 4-(pyridin-2-ylmethyl)morpholine 1a $(35.6 \mathrm{mg}, 0.2 \mathrm{mmol})$ and 1-bromo-4-tert-butylbenzene $(51.2 \mathrm{mg}, 41.6 \mu \mathrm{L}$, $0.24 \mathrm{mmol})$ with $5 \mathrm{~mol} \% \mathrm{Pd}(\mathrm{OAc})_{2} / 7.5 \mathrm{~mol} \%$ NIXANTPHOS. The crude product was purified by flash column chromatography on silica gel (hexanes:EtOAc $=50: 50$ ) to give the product 3a as a yellow oil (57.1 mg, 92\% yield). ${ }^{1} \mathrm{H} \mathrm{NMR}\left(500 \mathrm{MHz}, \mathrm{CDCl}_{3}\right) \delta 8.50(\mathrm{ddd}, J=5.0,2.0,1.0 \mathrm{~Hz}, 1 \mathrm{H}), 7.63-7.55$ (m, 2H), $7.39(\mathrm{~d}, J=8.4 \mathrm{~Hz}, 2 \mathrm{H}), 7.28(\mathrm{~d}, J=8.4 \mathrm{~Hz}, 2 \mathrm{H}), 7.07(\mathrm{ddd}, J=6.9,5.1,1.7 \mathrm{~Hz}, 1 \mathrm{H}), 4.38(\mathrm{~s}$, 1H), $3.72(\mathrm{t}, J=4.7 \mathrm{~Hz}, 4 \mathrm{H}), 2.50-2.28(\mathrm{~m}, 4 \mathrm{H}), 1.25(\mathrm{~s}, 9 \mathrm{H}) \mathrm{ppm} ;{ }^{13} \mathrm{C} \mathrm{NMR}\left(125 \mathrm{MHz}, \mathrm{CDCl}_{3}\right) \delta$ 162.0, 150.3, 149.2, 137.4, 136.8, 128.0, 125.6, 122.3, 122.1, 78.1, 67.2, 52.7, 34.5, 31.4 ppm; IR (neat) $3052,3006,2960,2905,2854,2811,2762,1716,1589,1570,1508,1469,1344,1395,1364,1280$, 1204, 1118, $1012 \mathrm{~cm}^{-1}$; HRMS m/z 225.1525 [(M-C $\left.{ }_{4} \mathrm{H}_{7} \mathrm{NO}\right)^{+}$; calcd for $\mathrm{C}_{16} \mathrm{H}_{19} \mathrm{~N}$ : 225.1517].

\section{Alternation: $53.4 \mathrm{mg}, 86 \%$ yield with $\mathrm{Pd}(\mathrm{OAc})_{2}(1 \mathrm{~mol} \%), \mathrm{NIXANTPHOS}(1.5 \mathrm{~mol} \%)$}

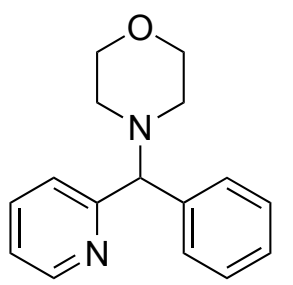

4-(Phenyl(pyridin-2-yl)methyl)morpholine (3b). The product was prepared by General Procedure B using 4-(pyridin-2-ylmethyl)morpholine 1a (35.6 mg, $0.2 \mathrm{mmol}$ ) and bromobenzene $(37.7 \mathrm{mg}, 25.4 \mu \mathrm{L}, 0.24 \mathrm{mmol})$ with $5 \mathrm{~mol} \% \mathrm{Pd}(\mathrm{OAc})_{2} / 7.5 \mathrm{~mol} \%$ NIXANTPHOS. The crude product was purified by flash column chromatography on silica gel 
(hexanes:EtOAc $=50: 50)$ to give the product $\mathbf{3 b}$ as a yellow solid $(41.2 \mathrm{mg}, 81 \%$ yield with bromobenzene), m.p. $=72-74{ }^{\circ} \mathrm{C} .{ }^{1} \mathrm{H} \mathrm{NMR}\left(500 \mathrm{MHz}, \mathrm{CDCl}_{3}\right) \delta 8.51(\mathrm{ddd}, J=5.0,2.0,1.0 \mathrm{~Hz}, 1 \mathrm{H})$, $7.62(\mathrm{tt}, J=7.5,1.5 \mathrm{~Hz}, 1 \mathrm{H}), 7.56(\mathrm{dd}, J=7.5,1.0 \mathrm{~Hz}, 1 \mathrm{H}), 7.50$ (d. $J=7.5 \mathrm{~Hz}, 2 \mathrm{H}), 7.31-7.25(\mathrm{~m}$, 2H), $7.23-7.17(\mathrm{~m}, 1 \mathrm{H}), 7.09(\mathrm{ddt}, J=7.0,5.0,1.0 \mathrm{~Hz}, 1 \mathrm{H}), 4.41(\mathrm{~s}, 1 \mathrm{H}), 3.73(\mathrm{t}, J=4.5 \mathrm{~Hz}, 4 \mathrm{H})$, $2.60-2.25(\mathrm{~m}, 4 \mathrm{H}) \mathrm{ppm} ;{ }^{13} \mathrm{C}$ NMR $\left(125 \mathrm{MHz}, \mathrm{CDCl}_{3}\right) \delta 161.9,149.5,140.8,136.9,128.8,128.6$ 127.6, 122.4, 122.3, 78.6, 67.2, 52.9 ppm; IR (neat) 3061, 2958, 2851, 2811, 2762, 1587, 1570, 1493, $1470,1451,1432,1395,1279,1246,1117,1070,1012 \mathrm{~cm}^{-1}$; HRMS m/z 255.1498 [(M+H) ${ }^{+}$; calcd for $\left.\mathrm{C}_{16} \mathrm{H}_{19} \mathrm{~N}_{2} \mathrm{O}: 255.1497\right]$.

Alternation: $39.7 \mathrm{mg}$, $78 \%$ yield with chlorobenzene $(27.0 \mathrm{mg}, 24.3 \mu \mathrm{L}, 0.24 \mathrm{mmol})$

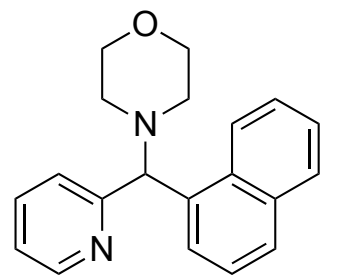

4-(Naphthalen-1-yl(pyridin-2-yl)methyl)morpholine (3c). The product was prepared by General Procedure B using 4-(pyridin-2-ylmethyl)morpholine 1a (35.6 $\mathrm{mg}, 0.2 \mathrm{mmol}$ ) and 1-bromonaphthalene (49.7 $\mathrm{mg}, 33.6 \mu \mathrm{L}, 0.24 \mathrm{mmol})$ with 5 mol\% $\mathrm{Pd}(\mathrm{OAc})_{2} / 7.5$ mol\% NIXANTPHOS. The crude product was purified by flash column chromatography on silica gel (hexanes:EtOAc $=50: 50)$ to afford the product $3 \mathbf{c}$ as a yellow solid (48.7 mg, 80\% yield), m.p. $=98-100{ }^{\circ} \mathrm{C} .{ }^{1} \mathrm{H}$ NMR $\left(300 \mathrm{MHz}, \mathrm{CDCl}_{3}\right) \delta 8.57(\mathrm{~d}, J=8.7 \mathrm{~Hz}, 1 \mathrm{H}), 8.51(\mathrm{ddd}, J$ $=4.8,1.5,1.2 \mathrm{~Hz}, 1 \mathrm{H}), 8.01(\mathrm{dd}, J=7.2,0.9 \mathrm{~Hz}, 1 \mathrm{H}), 7.81(\mathrm{ddd}, J=7.8,1.5,0.9 \mathrm{~Hz}, 1 \mathrm{H}), 7.74(\mathrm{~d}, J=$ $8.1 \mathrm{~Hz}, 1 \mathrm{H}), 7.58-7.39(\mathrm{~m}, 5 \mathrm{H}), 7.10-7.00(\mathrm{~m}, 1 \mathrm{H}) 5.29(\mathrm{~s}, 1 \mathrm{H}), 3.86-3.60(\mathrm{~m}, 4 \mathrm{H}), 2.70-2.50(\mathrm{~m}$, 2H), $2.50-2.30(\mathrm{~m}, 2 \mathrm{H}) \mathrm{ppm} ;{ }^{13} \mathrm{C} \mathrm{NMR}\left(75 \mathrm{MHz}, \mathrm{CDCl}_{3}\right) \delta 161.4,149.2,136.8,136.7,134.2,132.1$, $128.9,128.1,126.2,125.8,125.7,125.6,124.2,123.2$ 122.2, 73.9, 67.4, 53.1 ppm; IR (neat) 3050,2958 , 2853, 2811, 1588, 1570, 1511, 1452, 1432, 1396, 1310, 1275, $1117 \mathrm{~cm}^{-1} ; \mathrm{HRMS} \mathrm{m} / \mathrm{z} 305.1648$ $\left[(\mathrm{M}+\mathrm{H})^{+}\right.$; calcd for $\left.\mathrm{C}_{20} \mathrm{H}_{21} \mathrm{~N}_{2} \mathrm{O}: 305.1654\right]$. 


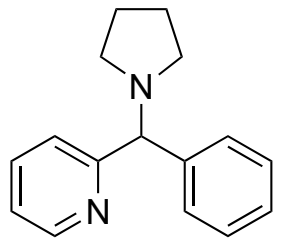

2-(Phenyl (pyrrolidin-1-yl)methyl)pyridine (3d). The product was prepared by General Procedure using 2-(pyrrolidin-1-ylmethyl)pyridine $1 \mathbf{b}(32.5 \mathrm{mg}, 0.20 \mathrm{mmol})$ and bromobenzene (37.7 mg, $25.3 \mu \mathrm{L}, 0.24 \mathrm{mmol})$ with $5 \mathrm{~mol} \% \mathrm{Pd}(\mathrm{OAc})_{2} / 7.5 \mathrm{~mol} \%$

NIXANTPHOS. The crude product was purified by flash column chromatography on silica gel (hexanes:EtOAc $=50: 50)$ to give the product 3d as a yellow solid $(39.6 \mathrm{mg}, 83 \%$ yield), m.p. $=76-78$ ${ }^{\circ} \mathrm{C} .{ }^{1} \mathrm{H}$ NMR $\left(500 \mathrm{MHz}, \mathrm{CDCl}_{3}\right) \delta 8.49(\mathrm{dt}, J=4.8,1.2 \mathrm{~Hz}, 1 \mathrm{H}), 7.61-7.53(\mathrm{~m}, 4 \mathrm{H}), 7.29-7.25(\mathrm{~m}$, 2H), $7.20-7.17(\mathrm{~m}, 1 \mathrm{H}), 7.07$ (ddd, $J=6.9,5.1,1.5 \mathrm{~Hz}, 1 \mathrm{H}), 4.37$ (s, 1H), $2.48-2.43(\mathrm{~m}, 4 \mathrm{H}), 1.80-$ $1.77(\mathrm{~m}, 4 \mathrm{H}) ;{ }^{13} \mathrm{C} \mathrm{NMR}\left(125 \mathrm{MHz}, \mathrm{CDCl}_{3}\right) \delta 163.3,149.2,142.9,136.8,128.6,128.1,127.3,122.2$, 122.1, 78.3, 53.6, 23.7. IR (neat) 3060, 3028, 3006, 2967, 2933, 2875, 2792, 1588, 1570, 1492, 1468, 1453, 1432, 1332, 1320, 1195, $1128 \mathrm{~cm}^{-1}$; HRMS m/z $239.1547\left[(\mathrm{M}+\mathrm{H})^{+}\right.$; calcd for $\mathrm{C}_{16} \mathrm{H}_{19} \mathrm{~N}_{2}$ : 239.1548].

Alternation: $35.8 \mathrm{mg}, 75 \%$ yield with $\mathrm{Pd}(\mathrm{OAc})_{2}$ (1 mol \%), NIXANTPHOS $(1.5 \mathrm{~mol} \%)$.

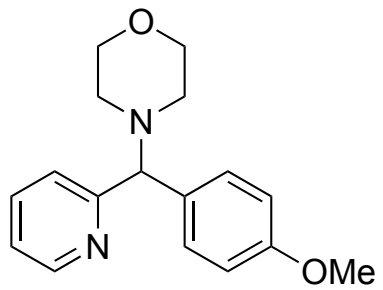

4-((4-Methoxyphenyl)(pyridin-2-yl)methyl)morpholine (3e). The product was prepared by General Procedure B using 4-(pyridin-2-ylmethyl)morpholine 1a $(35.6 \mathrm{mg}, 0.2 \mathrm{mmol})$ and 4-bromoanisole $(44.9 \mathrm{mg}, 30.1 \mu \mathrm{L}, 0.24 \mathrm{mmol})$ with $5 \mathrm{~mol} \% \mathrm{Pd}(\mathrm{OAc})_{2} / 7.5 \mathrm{~mol} \%$ NIXANTPHOS. The crude product was purified by flash column chromatography on silica gel (hexanes:EtOAc $=50: 50$ ) to give the product $3 \mathbf{e}$ as a yellow oil (46.1 mg, $81 \%$ yield). ${ }^{1} \mathrm{H}$ NMR $\left(300 \mathrm{MHz}, \mathrm{CDCl}_{3}\right) \delta 8.51$ (ddd, $\left.J=4.8,1.8,0.9 \mathrm{~Hz}, 1 \mathrm{H}\right)$, $7.62(\mathrm{td}, J=7.8,1.8 \mathrm{~Hz}, 1 \mathrm{H}), 7.54(\mathrm{~d}, J=7.8 \mathrm{~Hz}, 1 \mathrm{H}), 7.39$ (app d, $J=9.0 \mathrm{~Hz}, 2 \mathrm{H}), 7.09$ (ddd, $J=7.2$, 4.8, $1.5 \mathrm{~Hz}, 1 \mathrm{H}), 6.82(\mathrm{~d}, J=9.0 \mathrm{~Hz}, 2 \mathrm{H}), 4.36(\mathrm{~s}, 1 \mathrm{H}), 3.75(\mathrm{~s}, 3 \mathrm{H}), 3.72(\mathrm{t}, J=4.8 \mathrm{~Hz}, 4 \mathrm{H}), 2.54-2.30$ (m, 4H); ${ }^{13} \mathrm{C}$ NMR $\left(75 \mathrm{MHz}, \mathrm{CDCl}_{3}\right) \delta 162.3,159.1,149.5,136.9,132.9,129.6,122.3,122.2,114.2$, 77.9, 67.3, 55.4, 52.8 ppm; IR (neat) 3050, 3004, 2910, 2957, 2851, 2810, 1610, 1588, 1510, 1468, 
1432, 1289, 1250, 1174, 1117, 1034, $1010 \mathrm{~cm}^{-1}$; HRMS m/z 285.1599 [(M+H) ${ }^{+}$; calcd for $\mathrm{C}_{17} \mathrm{H}_{21} \mathrm{~N}_{2} \mathrm{O}_{2}$ : $285.1603]$.

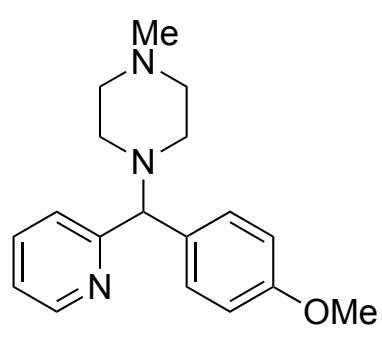
1-((4-Methoxyphenyl)(pyridin-2-yl)methyl)-4-methylpiperazine (3f). The product was prepared by General Procedure B using 1-methyl-4-(pyridin-2ylmethyl)piperazine 1c (38.3 $\mathrm{mg}, 0.20 \mathrm{mmol})$ and 4-bromoanisole (44.9 $\mathrm{mg}$, $30.1 \mu \mathrm{L}, 0.24 \mathrm{mmol})$ with $5 \mathrm{~mol} \% \operatorname{Pd}(\mathrm{OAc})_{2} / 7.5 \mathrm{~mol} \%$ NIXANTPHOS. The crude product was purified by flash column chromatography on silica gel $\left(\mathrm{CH}_{2} \mathrm{Cl}_{2}: \mathrm{CH}_{3} \mathrm{OH}=90: 10\right)$ to give the product $\mathbf{3 f}$ as a yellow oil $\left(55.9 \mathrm{mg}, 94 \%\right.$ yield). ${ }^{1} \mathrm{H} \mathrm{NMR}\left(500 \mathrm{MHz}, \mathrm{CDCl}_{3}\right) \delta 8.50(\mathrm{ddd}, J=$ 4.8, 1.8, 0.9 Hz, 1H), $7.61(\mathrm{td}, J=7.7,1.8 \mathrm{~Hz}, 1 \mathrm{H}), 7.51(\mathrm{~d}, J=8.0 \mathrm{~Hz}, 1 \mathrm{H}), 7.40-7.37(\mathrm{~m}, 2 \mathrm{H}), 7.08$ (ddd, $J=7.4,4.9,1.1 \mathrm{~Hz}, 1 \mathrm{H}), 6.83-6.80(\mathrm{~m}, 2 \mathrm{H}), 4.37(\mathrm{~s}, 1 \mathrm{H}), 3.75(\mathrm{~s}, 3 \mathrm{H}), 2.54(\mathrm{~m}, 8 \mathrm{H}), 2.33(\mathrm{~s}, 3 \mathrm{H})$ ppm; ${ }^{13} \mathrm{C}$ NMR $\left(125 \mathrm{MHz}, \mathrm{CDCl}_{3}\right) \delta 162.4,159.0,149.4,136.8,133.8,129.5,122.4,122.3,122.1$, 114.1, 77.2, 55.3, 55.1, 51.6, 45.7 ppm; IR (neat) 3036, 2936, 2804, 1609, 1588, 1569, 1510, 1461, 1433, 1291, 1248, 1175, 1143, 1033, $1008 \mathrm{~cm}^{-1}$; HRMS m/z 298.1921 [(M+H) ${ }^{+}$; calcd for $\mathrm{C}_{18} \mathrm{H}_{24} \mathrm{~N}_{3} \mathrm{O}$ : 298.1919].

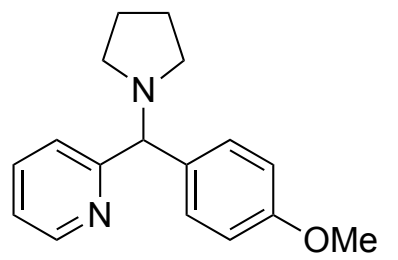

2-((4-Methoxyphenyl)(pyrrolidin-1-yl)methyl)pyridine (3g). The product was prepared by General Procedure B using 2-(pyrrolidin-1-ylmethyl)pyridine $1 \mathbf{b}(32.5 \mathrm{mg}, 0.20 \mathrm{mmol})$ and 4-bromoanisole $(44.9 \mathrm{mg}, 30.1 \mu \mathrm{L}, 0.24 \mathrm{mmol})$ with $5 \mathrm{~mol} \% \mathrm{Pd}(\mathrm{OAc})_{2} / 7.5 \mathrm{~mol} \%$ NIXANTPHOS. The crude product was purified by flash column chromatography on silica gel (hexanes:EtOAc $=50: 50)$ to give the product $\mathbf{3 g}$ as a yellow oil $(43.5 \mathrm{mg}$, $81 \%$ yield). ${ }^{1} \mathrm{H}$ NMR $\left(500 \mathrm{MHz}, \mathrm{CDCl}_{3}\right) \delta 8.49(\mathrm{~d}, J=4.5 \mathrm{~Hz}, 1 \mathrm{H}), 7.60(\mathrm{t}, J=7.6 \mathrm{~Hz}, 1 \mathrm{H}), 7.54(\mathrm{~d}, J=$ $7.9 \mathrm{~Hz}, 1 \mathrm{H}), 7.44(\mathrm{~d}, J=8.6 \mathrm{~Hz}, 2 \mathrm{H}), 7.06(\mathrm{t}, J=6.0 \mathrm{~Hz}, 1 \mathrm{H}), 6.81(\mathrm{~d}, J=8.6 \mathrm{~Hz}, 2 \mathrm{H}), 4.31(\mathrm{~s}, 1 \mathrm{H})$, 
$3.75(\mathrm{~s}, 3 \mathrm{H}), 2.44(\mathrm{~m}, 4 \mathrm{H}), 1.78(\mathrm{~m}, 4 \mathrm{H}) ;{ }^{13} \mathrm{C} \mathrm{NMR}\left(125 \mathrm{MHz}, \mathrm{CDCl}_{3}\right) \delta$ 163.5, 158.8, 149.2, 136.7, 135.1, 129.1, 122.0, 113.9, 77.5, 55.3, 53.6, 23.7; IR (neat) 3060, 3003, 2965, 2933, 2875, 2834, 2798, $1609,1588,1569,1510,1468,1433,1329,1302,1247,1174,1129,1105,1035 \mathrm{~cm}^{-1} ; \mathrm{HRMS} \mathrm{m} / \mathrm{z}$ 269.1658 [(M+H) ${ }^{+}$; calcd for $\left.\mathrm{C}_{17} \mathrm{H}_{21} \mathrm{~N}_{2} \mathrm{O}: 269.1654\right]$.

Alternation: $38.1 \mathrm{mg}, 71 \%$ yield with $\mathrm{Pd}(\mathrm{OAc})_{2}(1 \mathrm{~mol} \%)$, NXANTPHOS $(1.5 \mathrm{~mol} \%)$.

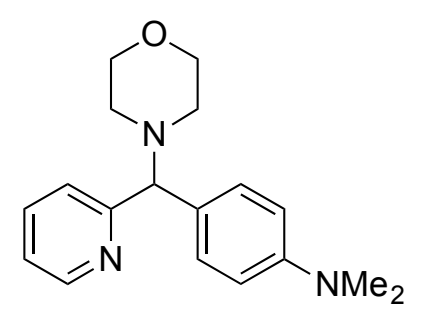

$N, N$-Dimethyl-4-(morpholino(pyridin-2-yl)methyl)aniline $\quad(3 \mathrm{~h}) . \quad$ The product was prepared by General Procedure B using 4-(pyridin-2ylmethyl)morpholine $\mathbf{1 a}(35.6 \mathrm{mg}, \quad 0.2 \mathrm{mmol})$ and 4-bromo-N,Ndimethylaniline (48.0 mg, $0.24 \mathrm{mmol})$ with $5 \mathrm{~mol} \% \mathrm{Pd}(\mathrm{OAc})_{2} / 7.5 \mathrm{~mol} \%$

NIXANTPHOS. The crude product was purified by flash column chromatography on silica gel (hexanes:EtOAc $=50: 50)$ to give the product $\mathbf{3 h}$ as a yellow solid $(46.4 \mathrm{mg}, 78 \%$ yield), m.p. $=101-$ $103{ }^{\circ} \mathrm{C} .{ }^{1} \mathrm{H}$ NMR $\left(500 \mathrm{MHz}, \mathrm{CDCl}_{3}\right) \delta 8.50(\mathrm{ddd}, J=4.8,1.8,0.9 \mathrm{~Hz}, 1 \mathrm{H}), 7.65-7.51(\mathrm{~m}, 2 \mathrm{H}), 7.37$ $(\operatorname{app~d}, J=9.0 \mathrm{~Hz}, 2 \mathrm{H}), 7.06(\mathrm{ddd}, J=6.9,4.8,1.5 \mathrm{~Hz}, 1 \mathrm{H}), 6.65(\operatorname{app~d}, J=9.0 \mathrm{~Hz}, 2 \mathrm{H}), 4.30(\mathrm{~s}, 1 \mathrm{H})$, $3.72(\mathrm{t}, J=4.8 \mathrm{~Hz}, 4 \mathrm{H}), 2.89(\mathrm{~s}, 6 \mathrm{H}), 2.51-2.30(\mathrm{~m}, 4 \mathrm{H}) \mathrm{ppm} ;{ }^{13} \mathrm{C} \mathrm{NMR}\left(125 \mathrm{MHz}, \mathrm{CDCl}_{3}\right) \delta 162.7$ $150.1,149.4,136.8,129.3,128.4,122.2,122.0,112.7,78.0,67.3,52.9,40.7$ ppm; IR (neat) 2955, 2923, $2852,2807,1613,1589,1569,1521,1470,1451,1432,1350,1281,1165,1118,1069,1014 \mathrm{~cm}^{-1}$ HRMS m/z 298.1913 [(M+H) ${ }^{+}$; calcd for $\left.\mathrm{C}_{18} \mathrm{H}_{24} \mathrm{~N}_{3} \mathrm{O}: 298.1919\right]$.

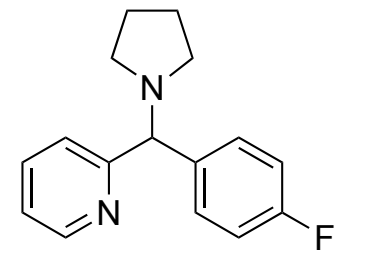

2-((4-Fluorophenyl)(pyrrolidin-1-yl)methyl)pyridine (3i). The product was prepared by General Procedure B using 2-(pyrrolidin-1-ylmethyl)pyridine 1b (32.5 with $5 \mathrm{~mol} \% \mathrm{Pd}(\mathrm{OAc})_{2} / 7.5 \mathrm{~mol} \%$ NIXANTPHOS. The crude product was purified by flash column 
chromatography on silica gel (hexanes:EtOAc $=50: 50)$ to give the product $\mathbf{3 i}$ as a yellow oil $(44.6 \mathrm{mg}$, 87\% yield). ${ }^{1} \mathrm{H}$ NMR $\left(500 \mathrm{MHz}, \mathrm{CDCl}_{3}\right) \delta 8.50(\mathrm{~d}, J=4.8 \mathrm{~Hz}, 1 \mathrm{H}), 7.61$ (tt, $\left.J=7.6,1.6 \mathrm{~Hz}, 1 \mathrm{H}\right), 7.54-$ $7.49(\mathrm{~m}, 3 \mathrm{H}), 7.10-7.07(\mathrm{~m}, 1 \mathrm{H}), 6.96(\mathrm{t}, J=8.5 \mathrm{~Hz}, 2 \mathrm{H}), 4.36(\mathrm{~s}, 1 \mathrm{H}), 2.44(\mathrm{~m}, 4 \mathrm{H}), 1.78(\mathrm{~m}, 4 \mathrm{H})$ ppm; ${ }^{13} \mathrm{C}$ NMR $\left(125 \mathrm{MHz}, \mathrm{CDCl}_{3}\right) \delta$ 163.0, 162.1 (d, $\left.J=243.8 \mathrm{~Hz}\right), 149.2,138.6(\mathrm{~d}, J=3.8 \mathrm{~Hz}), 136.8$, $129.5(\mathrm{~d}, J=8.8 \mathrm{~Hz}), 122.2,122.0,115.3(\mathrm{~d}, J=21.2 \mathrm{~Hz}), 77.3,53.5,23.7 \mathrm{ppm}$; IR (neat) 3051, 3007, 2967, 2876, 2798, 1603, 1589, 1570, 1506, 1469, 1433, 1417, 1362, 1324, 1293, 1220, 1155, 1128, 1093, 1049, $1015 \mathrm{~cm}^{-1}$; HRMS m/z 257.1450 [(M+H ${ }^{+}$; calcd for $\left.\mathrm{C}_{16} \mathrm{H}_{18} \mathrm{FN}_{2}: 257.1454\right]$.

Alternation: $36.4 \mathrm{mg}, 71 \%$ yield with $\mathrm{Pd}(\mathrm{OAc})_{2}(1 \mathrm{~mol} \%)$, NXANTPHOS $(1.5 \mathrm{~mol} \%)$.

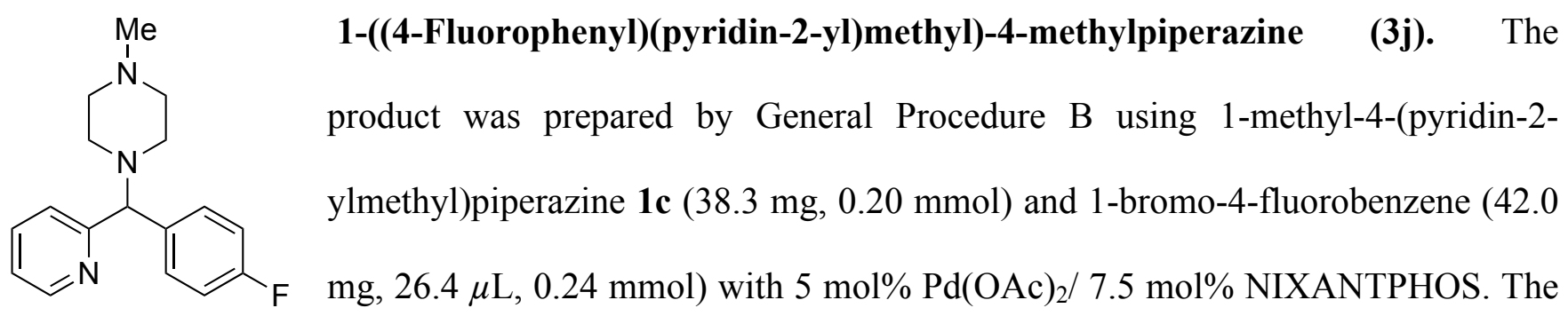
crude product was purified by flash column chromatography on silica gel $\left(\mathrm{CH}_{2} \mathrm{Cl}_{2}: \mathrm{CH}_{3} \mathrm{OH}=90: 10\right)$ to give the product $3 \mathbf{j}$ as a yellow oil (53.6 $\mathrm{mg}, 94 \%$ yield). ${ }^{1} \mathrm{H}$ NMR $\left(500 \mathrm{MHz}, \mathrm{CDCl}_{3}\right) \delta 8.51$ (ddd, $J=$ 5.0, 2.0, 1.0, Hz, 1H), $7.62(\mathrm{td}, J=7.8,1.7 \mathrm{~Hz}, 1 \mathrm{H}), 7.50(\mathrm{~d}, J=7.8 \mathrm{~Hz}, 1 \mathrm{H}), 7.45(\mathrm{dd}, J=9.0,5.5 \mathrm{~Hz}$, 2H), $7.10(\mathrm{ddd}, \mathrm{J}=7.0,5.0,1.0 \mathrm{~Hz}, 1 \mathrm{H}), 6.96(\mathrm{t}, J=9.0 \mathrm{~Hz}, 2 \mathrm{H}), 4.41(\mathrm{~s}, 1 \mathrm{H}), 2.85-2.15(\mathrm{~m}, 8 \mathrm{H}), 2.33$ (s, 3H); ${ }^{13} \mathrm{C}$ NMR $\left(125 \mathrm{MHz}, \mathrm{CDCl}_{3}\right) \delta 162.2(\mathrm{~d}, J=244.8 \mathrm{~Hz}), 161.7,149.6,136.9,136.8(\mathrm{~d}, J=3.2$ Hz), $129.9(\mathrm{~d}, J=8.1 \mathrm{~Hz}), 122.4,122.3,115.5(\mathrm{~d}, J=21.0 \mathrm{~Hz}), 77.0,55.1,51.5,45.7 \mathrm{ppm}$; IR (neat) 3067, 2961, 2941, 2807, 1603, 1591, 1571, 1507, 1471, 1459, 1434, 1292, 1221, 1158, 1145, 1095, $1050,1008 \mathrm{~cm}^{-1}$; HRMS m/z $286.1721\left[(\mathrm{M}+\mathrm{H})^{+}\right.$; calcd for $\left.\mathrm{C}_{17} \mathrm{H}_{21} \mathrm{FN}_{3}: 286.1720\right]$. 


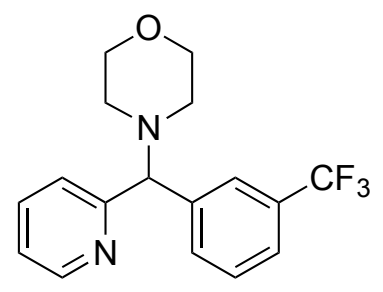

- The product was prepared by General Procedure B using 4-(pyridin-2ylmethyl)morpholine 1a (35.6 mg, $0.2 \mathrm{mmol})$ and 3-bromobenzotrifluoride $(54.0 \mathrm{mg}, \quad 33.5 \mu \mathrm{L}, 0.24 \mathrm{mmol})$ with $5 \mathrm{~mol} \% \mathrm{Pd}(\mathrm{OAc})_{2} / 7.5 \mathrm{~mol} \%$ NIXANTPHOS. The crude product was purified by flash column chromatography on silica gel (hexanes:EtOAc $=50: 50)$ to give the product $3 \mathbf{k}$ as a yellow oil $\left(54.2 \mathrm{mg}, 84 \%\right.$ yield). ${ }^{1} \mathrm{H}$ NMR (300 $\left.\mathrm{MHz}, \mathrm{CDCl}_{3}\right) \delta 8.54(\mathrm{ddd}, J=5.0,2.0,1.0, \mathrm{~Hz}, 1 \mathrm{H}), 7.78(\mathrm{~s}, 1 \mathrm{H}), 7.72(\mathrm{~d}, J=7.5 \mathrm{~Hz}, 1 \mathrm{H}), 7.65(\mathrm{td}, J=$ 7.5, 1.5 Hz, 1H), $7.53(\mathrm{~d}, J=8.0 \mathrm{~Hz}, 1 \mathrm{H}), 7.47(\mathrm{~d}, J=7.5 \mathrm{~Hz}, 1 \mathrm{H}), 7.41(\mathrm{t}, J=7.8 \mathrm{~Hz}, 1 \mathrm{H}), 7.13(\mathrm{ddd}, \mathrm{J}$ $=7.5,5.0,1.0 \mathrm{~Hz}, 1 \mathrm{H}), 4.50(\mathrm{~s}, 1 \mathrm{H}), 3.75(\mathrm{t}, J=4.8 \mathrm{~Hz}, 4 \mathrm{H}), 2.50-2.34(\mathrm{~m}, 4 \mathrm{H}),{ }^{13} \mathrm{C} \mathrm{NMR}(125 \mathrm{MHz}$, $\left.\mathrm{CDCl}_{3}\right) \delta 160.9,149.7,141.9,137.1,132.0,131.1(\mathrm{q}, J=32.0 \mathrm{~Hz}), 129.2,125.2(\mathrm{q}, J=3.8 \mathrm{~Hz}), 124.5$ (q, $J=3.8 \mathrm{~Hz}), 124.2(\mathrm{q}, J=270.7 \mathrm{~Hz}), 122.6,122.5,78.0,67.1,52.7$; IR (neat) 3054, 2959, 2854, 2813, 2769, 1588, 1571, 1470, 1449, 1433, 1327, 1277, 1166, 1118, 1073, $1014 \mathrm{~cm}^{-1} ;$ HRMS m/z $323.1358\left[(\mathrm{M}+\mathrm{H})^{+}\right.$; calcd for $\left.\mathrm{C}_{17} \mathrm{H}_{18} \mathrm{~N}_{2} \mathrm{OF}_{3}: 323.1371\right]$.

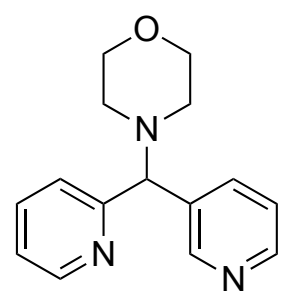

4- (Pyridin-2-yl(pyridin-3-yl)methyl)morpholine (3I). The product was prepared by General Procedure B using 4-(pyridin-2-ylmethyl)morpholine 1a (35.6 mg, 0.20 mmol) and 3-bromopyridine (37.9 mg, $23.1 \mu \mathrm{L}, 0.24 \mathrm{mmol})$ with $5 \mathrm{~mol} \% \mathrm{Pd}(\mathrm{OAc})_{2} /$ 7.5 mol\% NIXANTPHOS. The crude product was purified by flash column chromatography on silica gel hexanes:EtOAc $=60: 40$ to only EtOAc) to give the product $\mathbf{3 1}$ as a yellow oil (33.2 mg, 65\% yield). ${ }^{1} \mathrm{H}$ NMR (500 MHz, $\left.\mathrm{CDCl}_{3}\right) \delta 8.72(\mathrm{~d}, J=2.0 \mathrm{~Hz}, 1 \mathrm{H}), 8.55-8.54(\mathrm{~m}, 1 \mathrm{H})$, $8.47(\mathrm{dd}, J=4.8,1.5 \mathrm{~Hz}, 1 \mathrm{H}), 7.86(\mathrm{dt}, J=7.9,1.9 \mathrm{~Hz}, 1 \mathrm{H}), 7.66(\mathrm{td}, J=7.7,1.7 \mathrm{~Hz}, 1 \mathrm{H}), 7.53(\mathrm{~d}, J=$ $7.9 \mathrm{~Hz}, 1 \mathrm{H}), 7.23(\mathrm{dd}, J=7.9,4.7 \mathrm{~Hz}, 1 \mathrm{H}), 7.16-7.13(\mathrm{~m}, 1 \mathrm{H}), 4.47$ (s, 1H) 3.74 (t, $J=4.7 \mathrm{~Hz}, 4 \mathrm{H})$, $2.46-2.38(\mathrm{~m}, 4 \mathrm{H}) ;{ }^{13} \mathrm{C}$ NMR $\left(125 \mathrm{MHz}, \mathrm{CDCl}_{3}\right) \delta 160.6,150.1,149.8,149.1,137.1,136.3,136.2$ 123.8, 122.7, 122.6, 76.0, 67.1, 52.7; IR (neat) 3053, 2958, 2916, 2854, 2813, 1589, 1571, 1472, 1453, 
1433, 1396, 1305, 1286, 1273, 1251, 1211, 1117, 1070, 1029, $1012 \mathrm{~cm}^{-1} ; \mathrm{HRMS} \mathrm{m} / \mathrm{z} 256.1456$ $\left[(\mathrm{M}+\mathrm{H})^{+}\right.$; calcd for $\left.\mathrm{C}_{15} \mathrm{H}_{18} \mathrm{~N}_{3} \mathrm{O}: 256.1450\right]$.

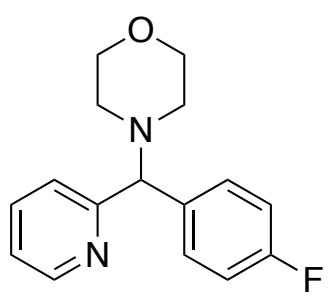

4-((4-Fluorophenyl)(pyridin-2-yl)methyl)morpholine (3m). The product was prepared by General Procedure using 4-(pyridin-2-ylmethyl)morpholine 1a (1.78 g, $10 \mathrm{mmol})$ and 1-bromo-4-fluorobenzene $(2.10 \mathrm{~g}, 1.32 \mathrm{~mL}, 12 \mathrm{mmol})$ with 4 $\operatorname{mol} \% \mathrm{Pd}(\mathrm{OAc})_{2} / 6 \mathrm{~mol} \%$ NIXANTPHOS. The crude product was purified by flash column chromatography on silica gel hexanes:EtOAc $=60: 40$ ) to give the product $\mathbf{3 m}$ as a brown oil (2.61 g, 96\% yield). ${ }^{1} \mathrm{H}$ NMR (500 MHz, $\left.\mathrm{CDCl}_{3}\right) \delta 8.50(\mathrm{ddd}, J=5.0,1.5,1.0 \mathrm{~Hz}, 1 \mathrm{H}), 7.61(\mathrm{td}, J=$ 7.5, 2.0 Hz, 1H), $7.51(\mathrm{~d}, J=8.0 \mathrm{~Hz}, 1 \mathrm{H}), 7.45(\mathrm{dd}, J=9.0,5.5 \mathrm{~Hz}, 2 \mathrm{H}), 7.09$ (ddd, $J=8.0,5.0,1.0 \mathrm{~Hz}$, 1H), $6.96(\mathrm{t}, J=9.0 \mathrm{~Hz}, 2 \mathrm{H}), 4.39(\mathrm{~s}, 1 \mathrm{H}), 3.71(\mathrm{t}, J=4.8 \mathrm{~Hz}, 4 \mathrm{H}), 2.50-2.28(\mathrm{~m}, 4 \mathrm{H}) ;{ }^{13} \mathrm{C}$ NMR $(125$ $\left.\mathrm{MHz}, \mathrm{CDCl}_{3}\right) \delta 162.2(\mathrm{~d}, J=244.5 \mathrm{~Hz}), 161.6,149.6,136.9,136.5(\mathrm{~d}, J=3.4 \mathrm{~Hz}), 130.0(\mathrm{~d}, J=8.0$ $\mathrm{Hz}), 122.35,122.31,115.6,(\mathrm{~d}, J=21.1 \mathrm{~Hz}), 77.7,67.2,52.7 ; \mathrm{HRMS} \mathrm{m} / \mathrm{z} 273.1398\left[(\mathrm{M}+\mathrm{H})^{+}\right.$; calcd for $\left.\mathrm{C}_{16} \mathrm{H}_{18} \mathrm{~N}_{2} \mathrm{OF}: 273.1403\right]$.

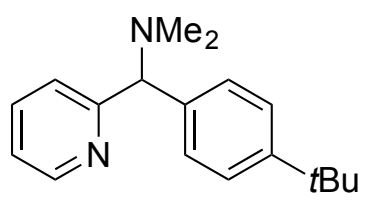

1-(4-(tert-Butyl)phenyl)- $N, N$-dimethyl-1-(pyridin-2-yl)methanamine

(4a).

The product was prepared by General Procedure B using $N, N$-dimethyl-1(pyridin-2-yl)methanamine 1d $(27.2 \mathrm{mg}, 0.20 \mathrm{mmol})$ and 1-bromo-4-tertbutylbenzene $(51.2 \mathrm{mg}, 41.6 \mu \mathrm{L}, 0.24 \mathrm{mmol})$ with $5 \mathrm{~mol} \% \mathrm{Pd}(\mathrm{OAc})_{2} / 7.5 \mathrm{~mol} \%$ NIXANTPHOS. The crude product was purified by flash column chromatography on silica gel (hexanes:EtOAc $=50: 50)$ to give the product $4 \mathbf{a}$ as a yellow solid $(51.0 \mathrm{mg}, 95 \%$ yield $)$, m.p. $=102-104{ }^{\circ} \mathrm{C} .{ }^{1} \mathrm{H} \mathrm{NMR}(500 \mathrm{MHz}$, $\left.\mathrm{CDCl}_{3}\right) \delta 8.51(\mathrm{dt}, J=4.9,0.9 \mathrm{~Hz}, 1 \mathrm{H}), 7.58(\mathrm{td}, J=7.6,1.7 \mathrm{~Hz}, 1 \mathrm{H}), 7.52(\mathrm{~d}, J=11.9 \mathrm{~Hz}, 1 \mathrm{H}), 7.42(\mathrm{~d}$, $J=8.4 \mathrm{~Hz}, 2 \mathrm{H}), 7.29(\mathrm{~d}, J=8.4 \mathrm{~Hz}, 2 \mathrm{H}), 7.06(\mathrm{ddd}, J=7.1,4.9,1.2 \mathrm{~Hz}, 1 \mathrm{H}), 4.23(\mathrm{~s}, 1 \mathrm{H}), 2.21(\mathrm{~s}, 6 \mathrm{H})$, 
$1.26(\mathrm{~s}, 9 \mathrm{H}) ;{ }^{13} \mathrm{C} \mathrm{NMR}\left(125 \mathrm{MHz}, \mathrm{CDCl}_{3}\right) \delta 163.1,150.2,149.3,138.9,136.7,127.9,125.5,122.3$ 120.0, 79.5, 44.9, 34.6, 31.5; IR (neat) 2953, 2862, 2816, 2770, 1586, 1568, 1507, 1467, 1435, 1363, 1254, 1180, 1154, 1118, $1030 \mathrm{~cm}^{-1}$; HRMS m/z 269.2018 [(M+H) ${ }^{+}$; calcd for $\left.\mathrm{C}_{18} \mathrm{H}_{25} \mathrm{~N}_{2}: 269.2018\right]$.

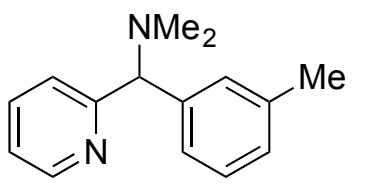

$N, N$-Dimethyl-1-(pyridin-2-yl)-1-(m-tolyl)methanamine (4b). The product

was prepared by General Procedure B using $N, N$-dimethyl-1-(pyridin-2yl)methanamine 1d (27.2 $\mathrm{mg}, 0.20 \mathrm{mmol})$ and 3-bromotoluene $(41.1 \mathrm{mg}, 29.1 \mu \mathrm{L}, 0.24 \mathrm{mmol})$ with 5 mol\% $\mathrm{Pd}(\mathrm{OAc})_{2} / 7.5 \mathrm{~mol} \%$ NIXANTPHOS. The crude product was purified by flash column chromatography on silica gel (hexanes:EtOAc $=50: 50)$ to give the product $\mathbf{4 b}$ as a yellow solid $(44.4$ mg, 98\% yield), m.p. $=88-90{ }^{\circ} \mathrm{C} .{ }^{1} \mathrm{H}$ NMR $\left(500 \mathrm{MHz}, \mathrm{CDCl}_{3}\right) \delta 8.51(\mathrm{~d}, J=4.8 \mathrm{~Hz}, 1 \mathrm{H}), 7.59(\mathrm{td}, J=$ 7.7, $1.5 \mathrm{~Hz}, 1 \mathrm{H}), 7.53(\mathrm{~d}, J=7.9 \mathrm{~Hz}, 1 \mathrm{H}), 7.33(\mathrm{~s}, 1 \mathrm{H}), 7.30(\mathrm{~d}, J=7.8 \mathrm{~Hz}, 1 \mathrm{H}), 7.16(\mathrm{t}, J=7.6 \mathrm{~Hz}, 1 \mathrm{H})$, $7.07-7.05(\mathrm{~m}, 1 \mathrm{H}), 7.00(\mathrm{~d}, J=7.4 \mathrm{~Hz}, 1 \mathrm{H}), 4.21(\mathrm{~s}, 1 \mathrm{H}), 2.30(\mathrm{~s}, 3 \mathrm{H}), 2.21(\mathrm{~s}, 6 \mathrm{H}) ;{ }^{13} \mathrm{C}$ NMR $(125$ $\left.\mathrm{MHz}, \mathrm{CDCl}_{3}\right) \delta 162.9,149.3,142.0,138.2,136.7,129.0,128.5,128.2,125.3,122.2,122.0,79.8,44.8$ 21.6; IR (neat) 2982, 2949, 2863, 2819, 2773, 1607, 1587, 1568, 1467, 1431, 1330, 1255, 1180, 1153, $1095,1028 \mathrm{~cm}^{-1}$; HRMS m/z 227.1545 [(M+H) ${ }^{+}$; calcd for $\left.\mathrm{C}_{15} \mathrm{H}_{18} \mathrm{~N}_{2}: 227.1548\right]$.

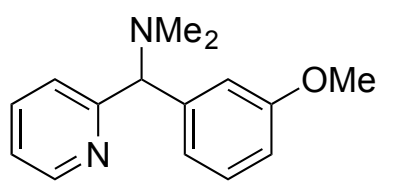

1-(3-Methoxyphenyl)- $N, \quad N$-dimethyl-1-(pyridin-2-yl)methanamine $\quad(4 c)$.

The product was prepared by General Procedure B using $N, N$-dimethyl-1(pyridin-2-yl)methanamine 1d $(27.2 \mathrm{mg}, 0.20 \mathrm{mmol})$ and 3-bromoanisole $(44.9 \mathrm{mg}, 30.4 \mu \mathrm{L}, 0.24$ mmol) with $5 \mathrm{~mol} \% \mathrm{Pd}(\mathrm{OAc})_{2} / 7.5 \mathrm{~mol} \%$ NIXANTPHOS. The crude product was purified by flash column chromatography on silica gel (hexanes:EtOAc $=50: 50$ ) to give the product $4 \mathbf{c}$ as a yellow oil (41.2 mg, 85\% yield). ${ }^{1} \mathrm{H} \mathrm{NMR}\left(500 \mathrm{MHz}, \mathrm{CDCl}_{3}\right) \delta 8.53-8.51(\mathrm{~m}, 1 \mathrm{H}), 7.60(\mathrm{dt}, J=7.7,1.7 \mathrm{~Hz}, 1 \mathrm{H})$, $7.52(\mathrm{~d}, J=8.0 \mathrm{~Hz}, 1 \mathrm{H}), 7.19(\mathrm{t}, J=7.7 \mathrm{~Hz}, 1 \mathrm{H}), 7.10-7.08(\mathrm{~m}, 3 \mathrm{H}), 6.75-6.73(\mathrm{~m}, 1 \mathrm{H}), 4.22(\mathrm{~s}, 1 \mathrm{H})$, 
$3.78(\mathrm{~s}, 3 \mathrm{H}), 2.22(\mathrm{~s}, 6 \mathrm{H}) ;{ }^{13} \mathrm{C}$ NMR $\left(125 \mathrm{MHz}, \mathrm{CDCl}_{3}\right) \delta 162.7,159.9,149.3,143.7,136.8,129.6$ $122.3,122.1,120.7,113.6,113.2,79.7,55.4,44.8$; IR (neat) 3053, 2987, 2952, 2859, 2821, 2775, 1587, 1569, 1488, 1470, 1455, 1434, 1319, 1282, 1257, 1181, 1147, 1094, $1043 \mathrm{~cm}^{-1} ; \mathrm{HRMS} \mathrm{m} / \mathrm{z} 243.1492$ $\left[(\mathrm{M}+\mathrm{H})^{+}\right.$; calcd for $\left.\mathrm{C}_{15} \mathrm{H}_{19} \mathrm{~N}_{2} \mathrm{O}: 243.1497\right]$.

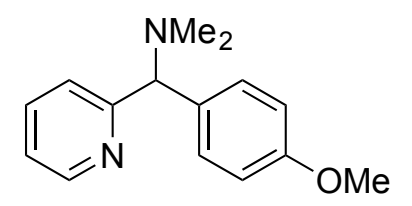

1-(4-Methoxyphenyl)- $N, \quad N$-dimethyl-1-(pyridin-2-yl)methanamine $\quad(4 d)$.

The product was prepared by General Procedure B using $N, N$-dimethyl-1(pyridin-2-yl)methanamine $1 \mathbf{d}(27.2 \mathrm{mg}, 0.20 \mathrm{mmol})$ and 4-bromoanisole (44.9 $\mathrm{mg}, 30.1 \mu \mathrm{L}, 0.24 \mathrm{mmol})$ with $5 \mathrm{~mol} \% \mathrm{Pd}(\mathrm{OAc})_{2} / 7.5 \mathrm{~mol} \%$ NIXANTPHOS. The crude product was purified by flash column chromatography on silica gel (hexanes:EtOAc $=50: 50$ ) to give the product $\mathbf{4 d}$ as a yellow oil (45.1 mg, 93\% yield). ${ }^{1} \mathrm{H}$ NMR (500 MHz, $\left.\mathrm{CDCl}_{3}\right) \delta 8.51(\mathrm{~d}, J=4.8 \mathrm{~Hz}, 1 \mathrm{H}), 7.60(\mathrm{t}, J=$ $7.7 \mathrm{~Hz}, 1 \mathrm{H}), 7.52(\mathrm{~d}, J=7.4 \mathrm{~Hz}, 1 \mathrm{H}), 7.41(\mathrm{~d}, J=8.5 \mathrm{~Hz}, 2 \mathrm{H}), 7.08-7.06(\mathrm{~m}, 1 \mathrm{H}), 6.82(\mathrm{~d}, J=8.5 \mathrm{~Hz}$ 2H), $4.30(\mathrm{~s}, 1 \mathrm{H}), 3.74(\mathrm{~s}, 3 \mathrm{H}), 2.21(\mathrm{~s}, 6 \mathrm{H}) ;{ }^{13} \mathrm{C} \mathrm{NMR}\left(125 \mathrm{MHz}, \mathrm{CDCl}_{3}\right) \delta 163.0,158.9,149.3,136.8$, 134.0, 129.4, 122.1, 122.0, 114.0, 78.8, 55.3, 44.7. IR (neat) 3051, 2988, 2951, 2863, 2818, 2773, 1610, $1588,1569,1508,1466,1433,1302,1284,1248,1179,1153,1109,1094,1037 \mathrm{~cm}^{-1} ; \mathrm{HRMS} \mathrm{m} / \mathrm{z}$ $243.1492\left[(\mathrm{M}+\mathrm{H})^{+}\right.$; calcd for $\left.\mathrm{C}_{15} \mathrm{H}_{19} \mathrm{~N}_{2} \mathrm{O}: 243.1497\right]$.

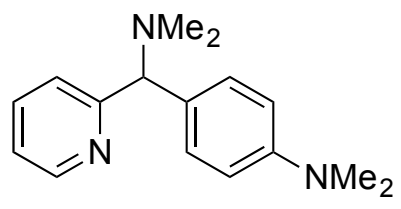

4-((Dimethylamino)(pyridin-2-yl)methyl)- $N, N$-dimethylaniline (4e). The product was prepared by General Procedure B using $N, N$-dimethyl-1(pyridin-2-yl)methanamine $\mathbf{1 d}(27.2 \mathrm{mg}, 0.20 \mathrm{mmol})$ and 4-bromo- $N, N$ dimethylaniline $(48.0 \mathrm{mg}, 0.24 \mathrm{mmol})$ with $5 \mathrm{~mol} \% \mathrm{Pd}(\mathrm{OAc})_{2} / 7.5 \mathrm{~mol} \%$ NIXANTPHOS. The crude product was purified by flash column chromatography on silica gel (hexanes:EtOAc $=50: 50$ ) to give the product $4 \mathbf{e}$ as a yellow solid $(47.0 \mathrm{mg}, 92 \%$ yield $)$, m.p. $=72-74{ }^{\circ} \mathrm{C} .{ }^{1} \mathrm{H} \mathrm{NMR}\left(500 \mathrm{MHz}, \mathrm{CDCl}_{3}\right) \delta$ 
$8.50(\mathrm{dt}, J=4.9,0.8 \mathrm{~Hz}, 1 \mathrm{H}), 7.58(\mathrm{td}, J=7.6,1.1 \mathrm{~Hz}, 1 \mathrm{H}), 7.51(\mathrm{dd}, J=7.9,0.8 \mathrm{~Hz}, 1 \mathrm{H}), 7.34(\mathrm{~d}, J=$

$8.5 \mathrm{~Hz}, 2 \mathrm{H}), 7.06-7.04(\mathrm{~m}, 1 \mathrm{H}), 6.65(\mathrm{~d}, J=8.5 \mathrm{~Hz}, 2 \mathrm{H}), 4.15(\mathrm{~s}, 1 \mathrm{H}), 2.89(\mathrm{~s}, 6 \mathrm{H}), 2.21(\mathrm{~s}, 6 \mathrm{H}) ;{ }^{13} \mathrm{C}$ $\operatorname{NMR}\left(125 \mathrm{MHz}, \mathrm{CDCl}_{3}\right) \delta 163.6,150.0,149.3,136.7,130.0,129.1,122.0,121.8,112.7,79.2,44.9$, 40.7; IR (neat) 3047, 2985, 2948, 2861, 2815, 2772, 1613, 1589, 1568, 1521, 1470, 1433, 1348, 1227 , 1185, 1163, $1025 \mathrm{~cm}^{-1}$; HRMS m/z 254.1669 (only peak) [(M-H) ${ }^{+}$; calcd for $\left.\mathrm{C}_{16} \mathrm{H}_{20} \mathrm{~N}_{3}: 254.1657\right]$.

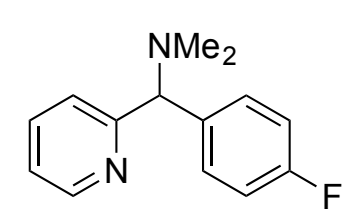

1-(4-Fluorophenyl)- $N, N$-dimethyl-1-(pyridin-2-yl)methanamine (4f). The product was prepared by General Procedure B using $N, N$-dimethyl-1-(pyridin-2-

yl)methanamine 1d $(27.2 \mathrm{mg}, 0.20 \mathrm{mmol})$ and 1-bromo-4-fluorobenzene (42.0 $\mathrm{mg}, 26.4 \mu \mathrm{L}, 0.24 \mathrm{mmol})$ with $5 \mathrm{~mol} \% \mathrm{Pd}(\mathrm{OAc})_{2} / 7.5 \mathrm{~mol} \%$ NIXANTPHOS. The crude product was purified by flash column chromatography on silica gel (hexanes:EtOAc $=50: 50$ ) to give the product $\mathbf{4 f}$ as a yellow oil (42.4 mg, 92\% yield). ${ }^{1} \mathrm{H}$ NMR (500 MHz, $\left.\mathrm{CDCl}_{3}\right) \delta 8.52(\mathrm{dt}, J=4.8,0.9 \mathrm{~Hz}, 1 \mathrm{H}), 7.61$ $(\mathrm{td}, J=7.7,1.7 \mathrm{~Hz}, 1 \mathrm{H}), 7.50-7.46(\mathrm{~m}, 3 \mathrm{H}), 7.11-7.09(\mathrm{~m}, 1 \mathrm{H}), 6.97(\mathrm{t}, J=8.6 \mathrm{~Hz}, 2 \mathrm{H}), 4.24(\mathrm{~s}, 1 \mathrm{H})$, $2.20(\mathrm{~s}, 6 \mathrm{H}) ;{ }^{13} \mathrm{C} \mathrm{NMR}\left(125 \mathrm{MHz}, \mathrm{CDCl}_{3}\right) \delta 162.6,162.2(\mathrm{~d}, J=244.0 \mathrm{~Hz}), 149.5,137.8(\mathrm{~d}, J=3.4 \mathrm{~Hz})$ 136.8, $129.8(\mathrm{~d}, J=7.8 \mathrm{~Hz}), 122.20,122.17,115.5$ (d, $J=21.1 \mathrm{~Hz}), 78.8,44.7$; IR (neat) 3052, 2982, 2952, 2867, 2821, 2776, 1602, 1589, 1570, 1507, 1471, 1433, 1325, 1293, 1221, 1179, 1156, 1097, 1041, $1027 \mathrm{~cm}^{-1}$; HRMS m/z 231.1301 [(M+H) ${ }^{+}$; calcd for $\mathrm{C}_{14} \mathrm{H}_{16} \mathrm{FN}_{2}$ : 231.1298].

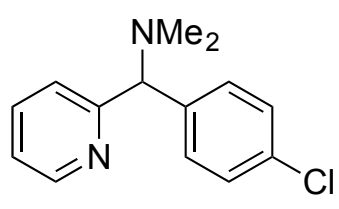

1-(4-Chlorophenyl)-N, N-dimethyl-1-(pyridin-2-yl)methanamine (4g). The product was prepared by General Procedure B using $N, N$-dimethyl-1-(pyridin-2yl)methanamine 1d (27.2 $\mathrm{mg}, 0.20 \mathrm{mmol})$ and 1-bromo-4-chlorobenzene $(46.0$ $\mathrm{mg}, 0.24 \mathrm{mmol})$ with $5 \mathrm{~mol} \% \mathrm{Pd}(\mathrm{OAc})_{2} / 7.5 \mathrm{~mol} \%$ NIXANTPHOS. The crude product was purified by flash column chromatography on silica gel (hexanes:EtOAc $=50: 50$ ) to give the product $\mathbf{4 g}$ as a yellow 
oil (47.4 mg, 96\% yield). ${ }^{1} \mathrm{H}$ NMR (500 MHz, $\left.\mathrm{CDCl}_{3}\right) \delta 8.52(\mathrm{dt}, J=4.9,0.9 \mathrm{~Hz}, 1 \mathrm{H}), 7.61(\mathrm{td}, J=7.7$, $1.7 \mathrm{~Hz}, 1 \mathrm{H}), 7.48$ (d, $J=7.9 \mathrm{~Hz}, 1 \mathrm{H}), 7.45$ (d, $J=8.4 \mathrm{~Hz}, 2 \mathrm{H}), 7.25$ (d, $J=8.9 \mathrm{~Hz}, 2 \mathrm{H}), 7.11-7.09$ (m, 1H), $4.24(\mathrm{~s}, 1 \mathrm{H}), 2.21(\mathrm{~s}, 6 \mathrm{H}) ;{ }^{13} \mathrm{C} \mathrm{NMR}\left(125 \mathrm{MHz}, \mathrm{CDCl}_{3}\right) \delta$ 162.3, 149.5, 140.6, 136.9, 133.1, 129.7, 128.8, 122.3, 122.2, 78.9, 44.7. IR (neat) 3050, 2987, 2952, 2865, 2821, 2776, 1588, 1570, 1487, 1470, $1432,1407,1325,1291,1253,1181,1152,1089,1028,1014 \mathrm{~cm}^{-1} ;$ HRMS m/z 247.1007 [(M+H) calcd for $\mathrm{C}_{14} \mathrm{H}_{16} \mathrm{ClN}_{2}$ : 247.1002].

(4h). The product was prepared by General Procedure B using N, N-dimethyl-1(pyridin-2-yl)methanamine 1d $(27.2 \mathrm{mg}, 0.20 \mathrm{mmol})$ and 3-bromobenzotrifluoride $(54.0 \mathrm{mg}, 33.5 \mu \mathrm{L}$, $0.24 \mathrm{mmol}$ ) with $5 \mathrm{~mol} \% \mathrm{Pd}(\mathrm{OAc})_{2} / 7.5 \mathrm{~mol} \%$ NIXANTPHOS. The crude product was purified by flash column chromatography on silica gel (hexanes:EtOAc $=50: 50$ ) to give the product $\mathbf{4 h}$ as a yellow solid (48.8 mg, 87\% yield), m.p. $=60-62{ }^{\circ} \mathrm{C} .{ }^{1} \mathrm{H}$ NMR $\left(500 \mathrm{MHz}, \mathrm{CDCl}_{3}\right) \delta 8.54(\mathrm{dt}, J=4.8,0.9 \mathrm{~Hz}, 1 \mathrm{H})$, 7.79 (s, 1H), $7.71(\mathrm{~d}, J=7.7 \mathrm{~Hz}, 1 \mathrm{H}), 7.63$ (td, $J=7.7,1.6 \mathrm{~Hz}, 1 \mathrm{H}), 7.48$ (dd, $J=7.8,1.4 \mathrm{~Hz}, 2 \mathrm{H}), 7.40$ (t, $J=7.7 \mathrm{~Hz}, 1 \mathrm{H}), 7.12(\mathrm{dd}, J=7.4,4.9 \mathrm{~Hz}, 1 \mathrm{H}), 4.33(\mathrm{~s}, 1 \mathrm{H}), 2.22(\mathrm{~s}, 6 \mathrm{H}) ;{ }^{13} \mathrm{C}$ NMR $(125 \mathrm{MHz}$, $\left.\mathrm{CDCl}_{3}\right) \delta 161.9,149.6,143.1,137.0,131.8,131.0(\mathrm{q}, J=37.5 \mathrm{~Hz}), 129.1,125.1(\mathrm{q}, J=3.8 \mathrm{~Hz}), 124.4$ (q, $J=271.0 \mathrm{~Hz}), 124.3(\mathrm{q}, J=3.8 \mathrm{~Hz}), 122.4,122.4,79.1,44.7$; IR (neat) 3056, 2989, 2955, 2868, $2825,2779,1673,1588,1571,1470,1450,1433,1326,1266,1165,1125,1098,1073,1042,1029 \mathrm{~cm}^{-1}$; HRMS m/z 281.1264 [(M+H $)^{+}$; calcd for $\left.\mathrm{C}_{15} \mathrm{H}_{16} \mathrm{~F}_{3} \mathrm{~N}_{2}: 281.1266\right]$.

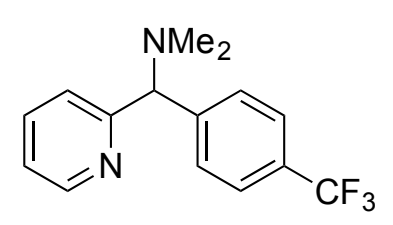

$N, N$-Dimethyl-1-(pyridin-2-yl)-1-(4-(trifluoromethyl)phenyl)methanamine

(4i). The product was prepared by General Procedure B using $N, N$-dimethyl-1(pyridin-2-yl)methanamine $\quad \mathbf{1 d} \quad(27.2 \quad \mathrm{mg}, \quad 0.20 \quad \mathrm{mmol}) \quad$ and $\quad 4-$ 
bromobenzotrifluoride $(54.0 \mathrm{mg}, \quad 33.6 \mu \mathrm{L}, \quad 0.24 \mathrm{mmol})$ with $5 \mathrm{~mol} \% \mathrm{Pd}(\mathrm{OAc})_{2} / 7.5 \mathrm{~mol} \%$ NIXANTPHOS. The crude product was purified by flash column chromatography on silica gel (hexanes:EtOAc $=50: 50)$ to give the product $4 \mathbf{i}$ as a yellow oil $\left(33.6 \mathrm{mg}, 60 \%\right.$ yield). ${ }^{1} \mathrm{H}$ NMR $(500$ $\left.\mathrm{MHz}, \mathrm{CDCl}_{3}\right) \delta 8.53(\mathrm{dt}, J=4.9,0.9 \mathrm{~Hz}, 1 \mathrm{H}), 7.65-7.61(\mathrm{~m}, 3 \mathrm{H}), 7.54(\mathrm{~d}, J=8.2 \mathrm{~Hz}, 2 \mathrm{H}), 7.50(\mathrm{~d}, J=$ $7.5 \mathrm{~Hz}, 1 \mathrm{H}), 7.12(\mathrm{dd}, J=7.4,4.9 \mathrm{~Hz}, 1 \mathrm{H}), 4.33(\mathrm{~s}, 1 \mathrm{H}), 2.21(\mathrm{~s}, 6 \mathrm{H}) ;{ }^{13} \mathrm{C} \mathrm{NMR}\left(125 \mathrm{MHz}, \mathrm{CDCl}_{3}\right) \delta$ $161.9,149.6,146.1,137.0,129.7(\mathrm{q}, J=32.1 \mathrm{~Hz}), 128.7,125.7(\mathrm{q}, J=3.7 \mathrm{~Hz}), 124.4(\mathrm{q}, J=270.5 \mathrm{~Hz})$, 122.5, 122.4, 79.2, 44.7. IR (neat) 3055, 2989, 2955, 2869, 2824, 2779, 1619, 1588, 1571, 1470, 1434, 1418, 1326, 1164, 1124, 1067, 1041, 1029, $1018 \mathrm{~cm}^{-1}$; HRMS m/z $281.1270\left[(\mathrm{M}+\mathrm{H})^{+}\right.$; calcd for $\left.\mathrm{C}_{15} \mathrm{H}_{16} \mathrm{~F}_{3} \mathrm{~N}_{2}: 281.1266\right]$.

1-(Benzofuran-5-yl)- $\mathbf{N}, \mathbf{N}$-dimethyl-1-(pyridin-2-yl)methanamine (4j). The yl)methanamine 1d $(27.2 \mathrm{mg}, 0.20 \mathrm{mmol})$ and 5-bromobenzofuran $(47.3 \mathrm{mg}, 30.1 \mu \mathrm{L}, 0.24 \mathrm{mmol})$ with $5 \mathrm{~mol} \% \mathrm{Pd}(\mathrm{OAc})_{2} / 7.5 \mathrm{~mol} \%$ NIXANTPHOS. The crude product was purified by flash column chromatography on silica gel (hexanes:EtOAc $=50: 50)$ to give the product $\mathbf{4 j}$ as a yellow oil $(33.3 \mathrm{mg}$, 66\% yield). ${ }^{1} \mathrm{H}$ NMR (500 MHz, $\left.\mathrm{CDCl}_{3}\right) \delta 8.51(\mathrm{~d}, J=4.8 \mathrm{~Hz}, 1 \mathrm{H}), 7.74(\mathrm{~s}, 1 \mathrm{H}), 7.62-7.56(\mathrm{~m}, 3 \mathrm{H})$, $7.47(\mathrm{~d}, J=8.5 \mathrm{~Hz}, 1 \mathrm{H}), 7.40(\mathrm{~d}, J=8.5 \mathrm{~Hz}, 1 \mathrm{H}), 7.08-7.05(\mathrm{~m}, 1 \mathrm{H}), 6.70(\mathrm{~s}, 1 \mathrm{H}), 4.35(\mathrm{~s}, 1 \mathrm{H}), 2.24$ $(\mathrm{s}, 6 \mathrm{H}) ;{ }^{13} \mathrm{C} \mathrm{NMR}\left(125 \mathrm{MHz}, \mathrm{CDCl}_{3}\right) \delta 163.2,154.5,149.4,145.4,136.8,127.7,124.6,122.2,122.0$ 120.9, 111.5, 106.9, 79.7, 44.9. IR (neat) 3053, 2986, 2950, 2862, 2820, 2774, 1588, 1569, 1465, 1432, 1327, 1261, 1181, 1153, 1124, 1108, $1030 \mathrm{~cm}^{-1}$; HRMS m/z 253.1336 [(M+H) ${ }^{+}$; calcd for $\mathrm{C}_{16} \mathrm{H}_{17} \mathrm{~N}_{2} \mathrm{O}$ : $253.1341]$. 


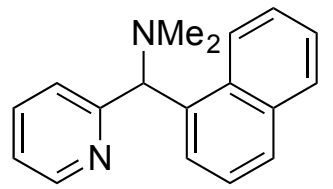

$N, \quad N$-Dimethyl-1-(naphthalen-1-yl)-1-(pyridin-2-yl)methanamine (4k). The

product was prepared by General Procedure B using $N, N$-dimethyl-1-(pyridin-2yl)methanamine 1d (27.2 mg, $0.20 \mathrm{mmol})$ and 1-bromonaphthalene (49.7 mg, 33.6 $\mu \mathrm{L}, 0.24 \mathrm{mmol})$ with $5 \mathrm{~mol} \% \mathrm{Pd}(\mathrm{OAc})_{2} / 7.5 \mathrm{~mol} \%$ NIXANTPHOS. The crude product was purified by flash column chromatography on silica gel (hexanes:EtOAc $=50: 50$ ) to give the product $\mathbf{4 k}$ as a yellow oil (36.2 mg, 69\% yield). ${ }^{1} \mathrm{H}$ NMR $\left(500 \mathrm{MHz}, \mathrm{CDCl}_{3}\right) \delta 8.58(\mathrm{~d}, J=8.6 \mathrm{~Hz}, 1 \mathrm{H}), 8.51(\mathrm{dd}, J=4.8,1.1$ Hz, 1H), $8.02(\mathrm{~d}, J=7.3 \mathrm{~Hz}, 1 \mathrm{H}), 7.80(\mathrm{~d}, J=8.1 \mathrm{~Hz}, 1 \mathrm{H}), 7.73(\mathrm{~d}, J=8.1 \mathrm{~Hz}, 1 \mathrm{H}), 7.53-7.48(\mathrm{~m}$, 4H), $7.42(\mathrm{t}, J=7.5 \mathrm{~Hz}, 1 \mathrm{H}), 7.04(\mathrm{q}, J=4.5 \mathrm{~Hz}, 1 \mathrm{H}), 5.13(\mathrm{~s}, 1 \mathrm{H}), 2.29(\mathrm{~s}, 6 \mathrm{H}) ;{ }^{13} \mathrm{C} \mathrm{NMR}(125 \mathrm{MHz}$, $\left.\mathrm{CDCl}_{3}\right) \delta 162.5,148.9,138.0,136.6,134.1,131.9,128.9,127.8,126.1,125.8,125.5,124.3,122.9$, 122.0, 74.9, 45.1. IR (neat) 3050, 2986, 2950, 2863, 2820, 2764, 1588, 1569, 1511, 1470, 1434, 1394, 1328, 1256, 1177, 1153, 1118, $1043 \mathrm{~cm}^{-1} ;$ HRMS m/z $263.1544\left[(\mathrm{M}+\mathrm{H})^{+}\right.$; calcd for $\mathrm{C}_{18} \mathrm{H}_{19} \mathrm{~N}_{2}$ : 263.1548].

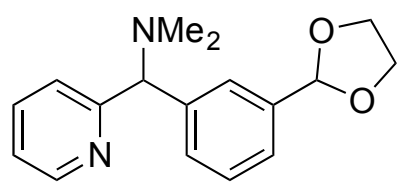

1-(3-(1,3-Dioxolan-2-yl)phenyl)- $N$,

$N$-dimethyl-1-(pyridin-2-

yl)methanamine (4I). The product was prepared by General Procedure B using $N, N$-dimethyl-1-(pyridin-2-yl)methanamine 1d (27.2 $\mathrm{mg}, 0.20 \mathrm{mmol}$ ) and 2-(3-bromophenyl)1,3-dioxolane (55.0 mg, $36.3 \mu \mathrm{L}, 0.24 \mathrm{mmol})$ with $5 \mathrm{~mol} \% \mathrm{Pd}(\mathrm{OAc})_{2} / 7.5 \mathrm{~mol} \%$ NIXANTPHOS. The crude product was purified by flash column chromatography on silica gel (hexanes:EtOAc $=50: 50$ ) to give the product $4 \mathrm{I}$ as a yellow oil $\left(50.1 \mathrm{mg}, 88 \%\right.$ yield). ${ }^{1} \mathrm{H}$ NMR $\left(500 \mathrm{MHz}, \mathrm{CDCl}_{3}\right) \delta 8.50(\mathrm{~d}, J=4.2$ $\mathrm{Hz}, 1 \mathrm{H}), 7.61-7.60(\mathrm{~m}, 1 \mathrm{H}), 7.58(\mathrm{~d}, J=7.6 \mathrm{~Hz}, 1 \mathrm{H}), 7.54(\mathrm{dd}, J=7.1,1.3 \mathrm{~Hz}, 1 \mathrm{H}), 7.51(\mathrm{~d}, J=7.8$ $\mathrm{Hz}, 1 \mathrm{H}), 7.37-7.28(\mathrm{~m}, 2 \mathrm{H}), 7.08-7.06(\mathrm{~m}, 1 \mathrm{H}), 5.76(\mathrm{~s}, 1 \mathrm{H}), 4.29(\mathrm{~s}, 1 \mathrm{H}), 4.13-4.07(\mathrm{~m}, 2 \mathrm{H}), 4.04$ - $3.98(\mathrm{~m}, 2 \mathrm{H}), 2.21(\mathrm{~s}, 6 \mathrm{H}) ;{ }^{13} \mathrm{C} \mathrm{NMR}\left(125 \mathrm{MHz}, \mathrm{CDCl}_{3}\right) \delta 162.5,149.3,142.2,138.2,136.8,129.2$, $128.8,126.6,125.6,122.3,122.1,103.9,79.6,65.5,65.4,44.8$; IR (neat) $3027,2961,2636,2836,2797$, 
$2691,1609,1596,1561,1510,1458,1413,1376,1301,1293,1249,1176,1160,1144,1105,1034,1008$ $\mathrm{cm}^{-1}$; HRMS m/z $285.1602\left[(\mathrm{M}+\mathrm{H})^{+}\right.$; calcd for $\mathrm{C}_{17} \mathrm{H}_{21} \mathrm{~N}_{2} \mathrm{O}_{2}$ : 285.1603].

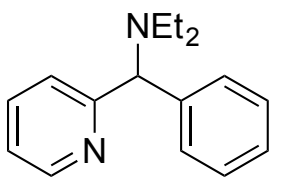

$N$-Ethyl- $N$-(phenyl(pyridin-2-yl)methyl)ethanamine $\quad(4 \mathrm{~m})$. The product was prepared by General Procedure B using $N$-ethyl- $N$-(pyridin-2-ylmethyl)ethanamine 1e (32.8 $\mathrm{mg}, 0.20 \mathrm{mmol}$ ) and bromobenzene $(37.7 \mathrm{mg}, 25.3 \mu \mathrm{L}, 0.24 \mathrm{mmol})$ with $5 \mathrm{~mol} \% \operatorname{Pd}(\mathrm{OAc})_{2} / 7.5$ mol\% NIXANTPHOS. The crude product was purified by flash column chromatography on silica gel ((hexanes:EtOAc $=50: 50)$ to give the product $\mathbf{4 m}$ as a yellow oil $(33.2 \mathrm{mg}, 69 \%$ yield). The NMR spectral data match the previously published data. ${ }^{9}$

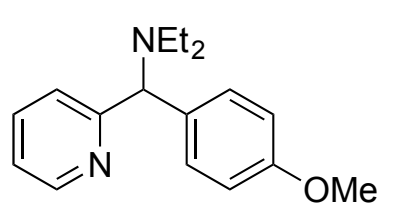

$N$-Ethyl- $N$-((4-methoxyphenyl)(pyridin-2-yl)methyl)ethanamine (4n). The product was prepared by General Procedure B using $N$-Ethyl- $N$-(pyridin-2ylmethyl)ethanamine 1e (32.8 mg, $0.20 \mathrm{mmol})$ and 4-bromoanisole $(44.9 \mathrm{mg}$, $30.0 \mu \mathrm{L}, 0.24 \mathrm{mmol})$ with $5 \mathrm{~mol} \% \mathrm{Pd}(\mathrm{OAc})_{2} / 7.5 \mathrm{~mol} \%$ NIXANTPHOS. The crude product was purified by flash column chromatography on silica gel ((hexanes:EtOAc $=50: 50)$ to give the product $4 \mathbf{n}$ as a yellow oil (35.6 mg, $66 \%$ yield). ${ }^{1} \mathrm{H}$ NMR $\left(500 \mathrm{MHz}, \mathrm{CDCl}_{3}\right) \delta 8.48(\mathrm{dd}, J=5.0,1.0 \mathrm{~Hz}, 1 \mathrm{H}), 7.61$ $-7.56(\mathrm{~m}, 2 \mathrm{H}), 7.40(\mathrm{~d}, J=8.8 \mathrm{~Hz}, 2 \mathrm{H}), 7.06(\mathrm{ddd}, J=6.7,4.9,1.8 \mathrm{~Hz}, 1 \mathrm{H}), 6.83-6.80(\mathrm{~m}, 2 \mathrm{H}), 4.81$ (s, 1H), $3.75(\mathrm{~s}, 3 \mathrm{H}), 2.65-2.54(\mathrm{~m}, 4 \mathrm{H}), 0.97(\mathrm{t}, J=7.1 \mathrm{~Hz}, 6 \mathrm{H}) ;{ }^{13} \mathrm{C}$ NMR $\left(125 \mathrm{MHz}, \mathrm{CDCl}_{3}\right) \delta$ 163.7, 158.7, 149.1, 136.6, 134.3, 129.6, 122.3, 121.8, 113.9, 73.0, 55.3, 42.9, 10.8. IR (neat) 3051, $2969,2932,2871,2835,1610,1588,1569,1509,1467,1432,1373,1302,1247,1173,1108,1036 \mathrm{~cm}^{-1}$ HRMS m/z $271.1801\left[(\mathrm{M}+\mathrm{H})^{+}\right.$; calcd for $\left.\mathrm{C}_{17} \mathrm{H}_{23} \mathrm{~N}_{2} \mathrm{O}: 271.1810\right]$. 


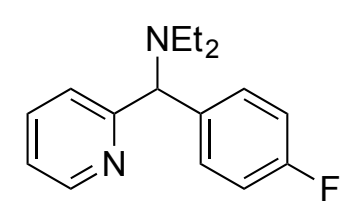

(40). The product was prepared by General Procedure B using $N$-ethyl- $N$-(pyridin-2-

ylmethyl)ethanamine 1e (32.8 $\mathrm{mg}, 0.20 \mathrm{mmol})$ and 1-bromo-4-fluorobenzene (42.0 mg, $26.4 \mu \mathrm{L}, 0.24 \mathrm{mmol}$ ) with $5 \mathrm{~mol} \% \operatorname{Pd}(\mathrm{OAc})_{2} / 7.5 \mathrm{~mol} \%$ NIXANTPHOS. The crude product was purified by flash column chromatography on silica gel ((hexanes:EtOAc $=50: 50)$ to give the product $4 \mathrm{o}$ as a yellow oil $\left(34.1 \mathrm{mg}, 66 \%\right.$ yield). ${ }^{1} \mathrm{H}$ NMR $\left(500 \mathrm{MHz}, \mathrm{CDCl}_{3}\right) \delta 8.49(\mathrm{~d}, J=4.8 \mathrm{~Hz}$, 1H), $7.62-7.59(\mathrm{~m}, 1 \mathrm{H}), 7.54(\mathrm{~d}, J=2.1 \mathrm{~Hz}, 1 \mathrm{H}), 7.46(\mathrm{dd}, J=5.7,8.4 \mathrm{~Hz}, 2 \mathrm{H}), 7.08-7.06(\mathrm{~m}, 1 \mathrm{H})$, $6.95(\mathrm{t}, J=8.7 \mathrm{~Hz} 2 \mathrm{H}), 4.86(\mathrm{~s}, 1 \mathrm{H}), 2.64-2.53(\mathrm{~m}, 4 \mathrm{H}), 0.97(\mathrm{t}, J=7.1 \mathrm{~Hz}, 6 \mathrm{H}) ;{ }^{13} \mathrm{C} \mathrm{NMR}(125 \mathrm{MHz}$, $\left.\mathrm{CDCl}_{3}\right) \delta 163.0,162.0(\mathrm{~d}, J=243.8 \mathrm{~Hz}), 149.2,137.9(\mathrm{~d}, J=2.5 \mathrm{~Hz}), 136.6,130.0(\mathrm{~d}, J=7.5 \mathrm{~Hz})$, 122.4, 122.0, 115.3 (d, $J=21.2 \mathrm{~Hz}$ ), 72.8, 42.9, 10.8; IR (neat) 3051, 2971, 2934, 2873, 2827, 1602, 1589, 1570, 1506, 1469, 1433, 1374, 1293, 1221, 1156, 1096, 1055, $1015 \mathrm{~cm}^{-1}$; HRMS m/z 259.1602 $\left[(\mathrm{M}+\mathrm{H})^{+}\right.$; calcd for $\left.\mathrm{C}_{16} \mathrm{H}_{20} \mathrm{FN}_{2}: 259.1611\right]$.

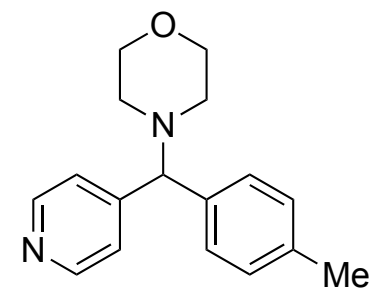

4-(Pyridin-4-yl(p-tolyl)methyl)morpholine (5a). The product was prepared by General Procedure B using 4-(pyridin-4-ylmethyl)morpholine 1f (35.6 mg, 0.20 mmol) and 4-bromotoluene (41.0 $\mathrm{mg}, 29.5 \mu \mathrm{L}, 0.24 \mathrm{mmol})$ with $1 \mathrm{~mol} \%$ $\mathrm{Pd}(\mathrm{OAc})_{2} / 1.5 \mathrm{~mol} \%$ NIXANTPHOS. The crude product was purified by flash column chromatography on silica gel (hexanes:EtOAc $=50: 50$ to only EtOAc) to give the product 5a as a yellow oil (48.8 mg, 91\% yield). ${ }^{1} \mathrm{H}$ NMR $\left(500 \mathrm{MHz}, \mathrm{CDCl}_{3}\right) \delta 8.49(\mathrm{~d}, J=5.5 \mathrm{~Hz}, 2 \mathrm{H}), 7.37$ (d, $J=$ $5.5 \mathrm{~Hz}, 2 \mathrm{H}), 7.24$ (d, $J=8.2 \mathrm{~Hz}, 2 \mathrm{H}), 7.10(\mathrm{~d}, J=8.2 \mathrm{~Hz}, 2 \mathrm{H}), 4.16(\mathrm{~s}, 1 \mathrm{H}), 3.71$ (t, $J=4.5 \mathrm{~Hz}, 4 \mathrm{H})$, $2.40-2.32(\mathrm{~m}, 4 \mathrm{H}), 2.29$ (s, 3H); ${ }^{13} \mathrm{C}$ NMR (125 MHz, $\left.\mathrm{CDCl}_{3}\right) \delta 151.9,150.2,137.7,137.5,129.7$, 128.2, 123.1, 75.6, 67.2, 52.6, 21.2; IR (neat) 3023, 2958, 2853, 2808, 1595, 1560, 1511, 1452, 1412, 1305, 1274, 1118, 1070, $1011 \mathrm{~cm}^{-1}$; HRMS m/z 268.1582 [(M) ${ }^{+}$; calcd for $\left.\mathrm{C}_{17} \mathrm{H}_{20} \mathrm{~N}_{2} \mathrm{O}: 268.1576\right]$. 


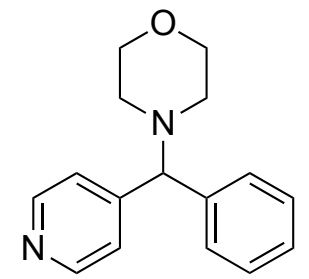

4-(Phenyl(pyridin-4-yl)methyl)morpholine (5b). The product was prepared by General Procedure B using 4-(pyridin-4-ylmethyl)morpholine 1f (35.6 mg, 0.20 mmol) and bromobenzene (37.7 mg, $25.4 \mu \mathrm{L}, 0.24 \mathrm{mmol})$ with $1 \mathrm{~mol} \% \mathrm{Pd}(\mathrm{OAc})_{2} /$ $1.5 \mathrm{~mol} \%$ NIXANTPHOS. The crude product was purified by flash column chromatography on silica gel (hexanes:EtOAc $=50: 50$ to only EtOAc) to give the product $5 \mathbf{b}$ as a yellow oil ( $46.8 \mathrm{mg}, 92 \%$ yield). ${ }^{1} \mathrm{H}$ NMR (500 MHz, CDCl $) \delta 8.49(\mathrm{dd}, J=5.0,1.5 \mathrm{~Hz}, 2 \mathrm{H}), 7.40-7.35(\mathrm{~m}, 4 \mathrm{H}), 7.29$ (app t, $J=7.0$ $\mathrm{Hz}, 2 \mathrm{H}), 7.22$ (app. t, $J=7.0 \mathrm{~Hz}, 1 \mathrm{H}), 4.20(\mathrm{~s}, 1 \mathrm{H}), 3.72(\mathrm{t}, J=5.0 \mathrm{~Hz}, 4 \mathrm{H}), 2.42-2.35(\mathrm{~m}, 4 \mathrm{H}) ;{ }^{13} \mathrm{C}$ NMR (125 MHz, $\left.\mathrm{CDCl}_{3}\right) \delta 151.6,150.3,140.6,129.0,128.3,128.0,123.2,75.9,67.3,52.7$; IR (neat) $3028,2959,2853,2810,1724,1668,1593,1361,1493,1451,1412,1306,1279,1117,1070,1011 \mathrm{~cm}^{-1}$ HRMS m/z $255.1502\left[(\mathrm{M}+\mathrm{H})^{+}\right.$; calcd for $\left.\mathrm{C}_{16} \mathrm{H}_{19} \mathrm{~N}_{2} \mathrm{O}: 255.1497\right]$.

Alternation: $43.2 \mathrm{mg}, 85 \%$ yield with chlorobenzene $(27.0 \mathrm{mg}, 24.3 \mu \mathrm{L}, 0.24 \mathrm{mmol}), \mathrm{Pd}(\mathrm{OAc})_{2}(5 \mathrm{~mol}$ \%), NIXANTPHOS (7.5 mol \%).

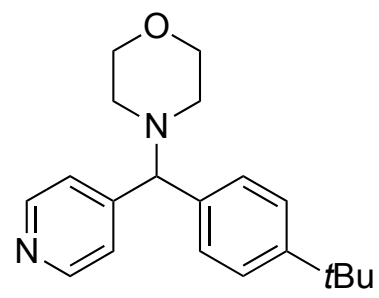

4-((4-(tert-Butyl)phenyl)(pyridin-4-yl)methyl)morpholine (5c). The product was prepared by General Procedure B using 4-(pyridin-4-ylmethyl)morpholine 1f (35.6 mg, $0.20 \mathrm{mmol})$ and 1-bromo-4-(tert-butyl)benzene (51.2 $\mathrm{mg}, 41.6 \mu \mathrm{L}$, $0.24 \mathrm{mmol})$ with $1 \mathrm{~mol} \% \mathrm{Pd}(\mathrm{OAc})_{2} / 1.5 \mathrm{~mol} \%$ NIXANTPHOS. The crude product was purified by flash column chromatography on silica gel (hexanes:EtOAc $=50: 50$ to only EtOAc) to give the product $\mathbf{5 c}$ as a white solid (52.8 mg, 85\% yield), m.p. $=82-84{ }^{\circ} \mathrm{C} .{ }^{1} \mathrm{H}$ NMR $(500$ $\left.\mathrm{MHz}, \mathrm{CDCl}_{3}\right) \delta 8.49(\mathrm{dd}, J=4.5,1.5 \mathrm{~Hz}, 2 \mathrm{H}), 7.38(\mathrm{dd}, J=4.5,1.5 \mathrm{~Hz}, 2 \mathrm{H}), 7.32-7.25(\mathrm{~m}, 4 \mathrm{H}), 4.17$ (s, 1H), $3.71(\mathrm{t}, J=4.5 \mathrm{~Hz}, 4 \mathrm{H}), 2.43-2.31(\mathrm{~m}, 4 \mathrm{H}), 1.27(\mathrm{~s}, 9 \mathrm{H}) ;{ }^{13} \mathrm{C}$ NMR $\left(125 \mathrm{MHz}, \mathrm{CDCl}_{3}\right) \delta$ 151.8, 150.8, 150.2, 137.3, 127.9, 125.8, 123.2, 75.5, 67.2, 52.6, 34.6, 31.4; IR (neat) 3027, 2961, 2906, 
2855, 2808, 1595, 1561, 1510, 1395, 1414, 1364, 1305, 1274, $1116 \mathrm{~cm}^{-1}$; HRMS m/z 311.2121 $\left[(\mathrm{M}+\mathrm{H})^{+}\right.$; calcd for $\mathrm{C}_{20} \mathrm{H}_{27} \mathrm{~N}_{2} \mathrm{O}$ : 311.2123$]$.

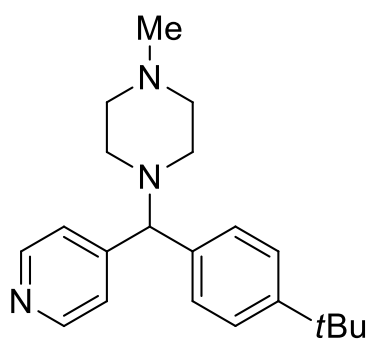

\author{
1-((4-(tert-Butyl)phenyl)(pyridin-4-yl)methyl)-4-methylpiperazine (5d). The \\ product was prepared by General Procedure B using 1-methyl-4-(pyridin-4- \\ ylmethyl)piperazine $\quad \mathbf{1 g} \quad(38.3 \mathrm{mg}, \quad 0.20 \mathrm{mmol})$ and 1-bromo-4-(tert- \\ butyl)benzene (51.2 mg, $41.6 \mu \mathrm{L}, 0.24 \mathrm{mmol})$ with $1 \mathrm{~mol} \% \operatorname{Pd}(\mathrm{OAc})_{2} / 1.5$
} mol\% NIXANTPHOS. The crude product was purified by flash column chromatography on silica gel (hexanes:EtOAc $=50: 50$ to only EtOAc) to give the product $\mathbf{5 d}$ as a yellow oil (52.4 $\mathrm{mg}, 81 \%$ yield). ${ }^{1} \mathrm{H}$ NMR (500 MHz, $\mathrm{CDCl}_{3}$ ) $\delta 8.50$ (app d., $\left.J=6.5 \mathrm{~Hz}, 2 \mathrm{H}\right), 7.36$ (app. d, $\left.J=5.6 \mathrm{~Hz}, 2 \mathrm{H}\right), 7.30$ (d, $J=$ $8.0 \mathrm{~Hz}, 2 \mathrm{H}), 7.25(\mathrm{~d}, J=8.0 \mathrm{~Hz}, 2 \mathrm{H}), 4.24(\mathrm{~s}, 1 \mathrm{H}), 2.90-2.20(\mathrm{~m}, 8 \mathrm{H}), 2.42(\mathrm{~s}, 3 \mathrm{H}), 1.27(\mathrm{~s}, 9 \mathrm{H}) ;{ }^{13} \mathrm{C}$ NMR (125 MHz, $\left.\mathrm{CDCl}_{3}\right) \delta 151.9,150.9,150.3,137.3,127.8,125.9,123.1,74.8,55.2,51.0,45.4,34.7$, 31.5; IR (neat) 3026, 2963, 2797, 1595, 1561, 1509, 1458, 1414, 1292, 1160, 1143, 1113, $1009 \mathrm{~cm}^{-1}$; HRMS m/z $324.2424\left[(\mathrm{M}+\mathrm{H})^{+}\right.$; calcd for $\left.\mathrm{C}_{21} \mathrm{H}_{30} \mathrm{~N}_{3}: 324.2440\right]$.

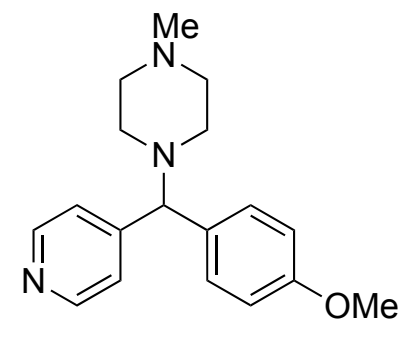

1-((4-Methoxyphenyl)(pyridin-4-yl)methyl)-4-methylpiperazine (5e). The product was prepared by General Procedure B using 1-methyl-4-(pyridin-4ylmethyl)piperazine $1 \mathrm{~g}$ (38.3 $\mathrm{mg}, 0.20 \mathrm{mmol})$ and 4-bromoanisole $(44.9 \mathrm{mg}$, $30.0 \mu \mathrm{L}, 0.24 \mathrm{mmol})$ with $5 \mathrm{~mol} \% \mathrm{Pd}(\mathrm{OAc})_{2} / 7.5 \mathrm{~mol} \%$ NIXANTPHOS. The crude product was purified by flash column chromatography on silica gel $\left(\mathrm{CH}_{2} \mathrm{Cl}_{2}: \mathrm{CH}_{3} \mathrm{OH}=90: 10\right)$ to give the product 5e as a yellow oil (52.3 mg, $88 \%$ yield). ${ }^{1} \mathrm{H}$ NMR $\left(500 \mathrm{MHz}, \mathrm{CDCl}_{3}\right) \delta 8.48(\mathrm{~d}, J=5.5$ $\mathrm{Hz}, 2 \mathrm{H}), 7.34(\mathrm{~d}, J=5.6 \mathrm{~Hz}, 2 \mathrm{H}), 7.25(\mathrm{~d}, J=8.6 \mathrm{~Hz}, 2 \mathrm{H}), 6.81(\mathrm{~d}, J=8.6 \mathrm{~Hz}, 2 \mathrm{H}), 4.17(\mathrm{~s}, 1 \mathrm{H}), 3.75$ (s, 3H), $2.50-2.43(\mathrm{~m}, 8 \mathrm{H}), 2.31(\mathrm{~s}, 3 \mathrm{H}) ;{ }^{13} \mathrm{C} \mathrm{NMR}\left(125 \mathrm{MHz}, \mathrm{CDCl}_{3}\right) \delta$ 159.1, 152.3, 150.1, 132.8, 
129.2, 122.9, 114.2, 74.5, 55.3, 55.2, 51.5, 45.7. IR (neat) 3027, 2961, 2936, 2836, 2797, 2691, 1609, $1596,1561,1510,1458,1413,1376,1301,1293,1249,1176,1160,1144,1105,1034,1008 \mathrm{~cm}^{-1}$ HRMS m/z 298.1924 [(M+H $)^{+}$; calcd for $\left.\mathrm{C}_{18} \mathrm{H}_{24} \mathrm{~N}_{3} \mathrm{O}: 298.1919\right]$.

Alternation: $44.0 \mathrm{mg}, 74 \%$ yield with Pd(OAc) 2 (1 mol \%), NIXANTPHOS $(1.5 \mathrm{~mol} \%)$.

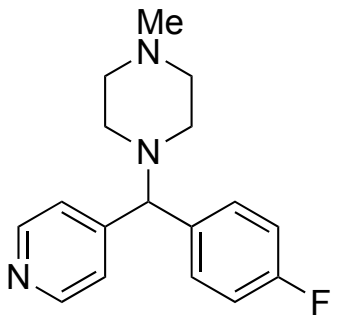

\section{1-((4-Fluorophenyl)(pyridin-4-yl)methyl)-4-methylpiperazine $\quad(5 f) . \quad$ The} product was prepared by General Procedure B using 1-methyl-4-(pyridin-4ylmethyl)piperazine $\mathbf{1 g}$ (38.3 mg, $0.20 \mathrm{mmol})$ and 1-bromo-4-fluorobenzene (42.0 $\mathrm{mg}, 26.4 \mu \mathrm{L}, 0.24 \mathrm{mmol})$ with $5 \mathrm{~mol} \% \operatorname{Pd}(\mathrm{OAc})_{2} / 7.5 \mathrm{~mol} \%$ NIXANTPHOS. The crude product was purified by flash column chromatography on silica gel $\left(\mathrm{CH}_{2} \mathrm{Cl}_{2}: \mathrm{CH}_{3} \mathrm{OH}=90: 10\right)$ to give the product $\mathbf{5 f}$ as a yellow oil $\left(48.5 \mathrm{mg}, 85 \%\right.$ yield). ${ }^{1} \mathrm{H}$ NMR $\left(500 \mathrm{MHz}, \mathrm{CDCl}_{3}\right) \delta 8.49(\mathrm{dd}, J=$ 4.5, 1.5 Hz, 2H), $7.33-7.31(\mathrm{~m}, 4 \mathrm{H}), 6.98(\mathrm{t}, J=8.6 \mathrm{~Hz}, 2 \mathrm{H}), 4.22(\mathrm{~s}, 1 \mathrm{H}), 2.51-2.42(\mathrm{~m}, 8 \mathrm{H}), 2.32(\mathrm{~s}$, 3H) ppm; ${ }^{13} \mathrm{C}$ NMR $\left(125 \mathrm{MHz}, \mathrm{CDCl}_{3}\right) \delta 162.3(\mathrm{~d}, J=245.1 \mathrm{~Hz}), 151.6,150.3,136.5(\mathrm{~d}, J=3.2 \mathrm{~Hz})$, $129.7(\mathrm{~d}, J=7.8 \mathrm{~Hz}), 123.0,115.9(\mathrm{~d}, J=21.4 \mathrm{~Hz}), 74.4,55.2,51.4,45.7 \mathrm{ppm}$; IR (neat) 3070, 2964, $2939,2805,2694,1600,1563,1507,1458,1415,1377,1293,1222,1158,1144,1095,1065,1009 \mathrm{~cm}^{-1}$; HRMS m/z $286.1719\left[(\mathrm{M}+\mathrm{H})^{+}\right.$; calcd for $\mathrm{C}_{17} \mathrm{H}_{21} \mathrm{FN}_{3}$ : 286.1720].

Alternation: $31.4 \mathrm{mg}$, $55 \%$ yield with Pd(OAc) 2 (1 mol \%), NIXANTPHOS $(1.5 \mathrm{~mol} \%)$.

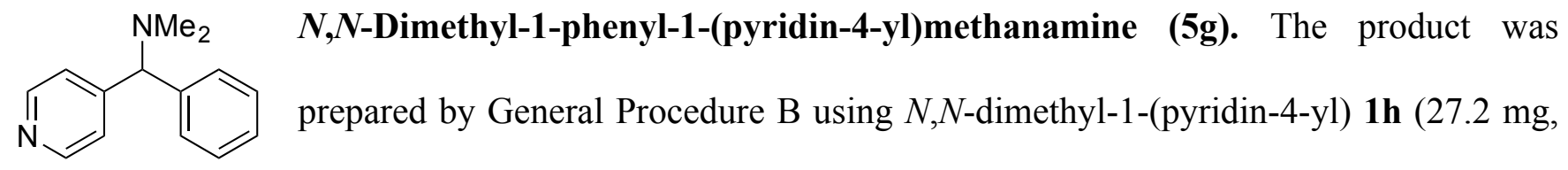
$0.20 \mathrm{mmol})$ and bromobenzene $(37.7 \mathrm{mg}, 25.4 \mu \mathrm{L}, 0.24 \mathrm{mmol})$ with $1 \mathrm{~mol} \% \mathrm{Pd}(\mathrm{OAc})_{2} / 1.5 \mathrm{~mol} \%$ NIXANTPHOS. The crude product was purified by flash column chromatography on silica gel 
(hexanes:EtOAc $=50: 50$ to only EtOAc) to give the product $\mathbf{5 g}$ as a yellow oil $\left(26.7 \mathrm{mg}, 63 \%\right.$ yield). ${ }^{1} \mathrm{H}$ $\operatorname{NMR}\left(500 \mathrm{MHz}, \mathrm{CDCl}_{3}\right) \delta 8.49(\mathrm{dd}, J=4.5,15 \mathrm{~Hz}, 2 \mathrm{H}), 7.39-7.35(\mathrm{~m}, 4 \mathrm{H}), 7.29(\mathrm{t}, J=7.8 \mathrm{~Hz}, 2 \mathrm{H})$, $7.24-7.18(\mathrm{~m}, 1 \mathrm{H}), 4.07(\mathrm{~s}, 1 \mathrm{H}), 2.19(\mathrm{~s}, 6 \mathrm{H}) ;{ }^{13} \mathrm{C} \mathrm{NMR}\left(125 \mathrm{MHz}, \mathrm{CDCl}_{3}\right) \delta 152.5,150.2,141.7$, 128.9, 128.1, 127.7, 123.0, 77.1, 44.6; IR (neat) 3062, 3026, 2987, 2952, 2864, 2820, 2775, 1594, 1561, 1492, 1454, 1411, 1310, 1180, 1152, 1040, $1022 \mathrm{~cm}^{-1}$; HRMS m/z $213.1389\left[(\mathrm{M}+\mathrm{H})^{+}\right.$; calcd for $\left.\mathrm{C}_{14} \mathrm{H}_{17} \mathrm{~N}_{2}: 213.1392\right]$.

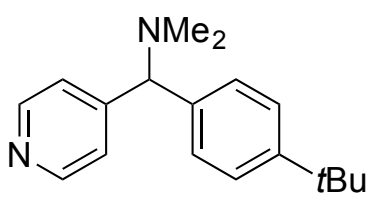

\section{1-(4-(tert-Butyl)phenyl)- $N, N$-dimethyl-1-(pyridin-4-yl)methanamine}

(5h).

The product was prepared by General Procedure B using N,N-dimethyl-1(pyridin-4-yl) $\mathbf{1 h}(27.2 \mathrm{mg}, 0.20 \mathrm{mmol})$ and 1-bromo-4-(tert-butyl)benzene (51.2 $\mathrm{mg}, 41.6 \mu \mathrm{L}, 0.24 \mathrm{mmol})$ with $1 \mathrm{~mol} \% \operatorname{Pd}(\mathrm{OAc})_{2} / 1.5 \mathrm{~mol} \%$ NIXANTPHOS. The crude product was purified by flash column chromatography on silica gel (hexanes:EtOAc $=50: 50$ to only EtOAc) to give the product $5 \mathbf{h}$ as a white solid $\left(36.5 \mathrm{mg}, 68 \%\right.$ yield), m.p. $=70-72{ }^{\circ} \mathrm{C} .{ }^{1} \mathrm{H}$ NMR $(500 \mathrm{MHz}$, $\mathrm{CDCl}_{3}$ ) $\delta 8.49$ (app. d, $J=6.0 \mathrm{~Hz}, 2 \mathrm{H}$ ), 7.37 (app. d, $J=6.0 \mathrm{~Hz}, 2 \mathrm{H}$ ), $7.32-7.26$ (m, 4H), 4.04 (s, 1H), $2.18(\mathrm{~s}, 6 \mathrm{H}), 1.27(\mathrm{~s}, 9 \mathrm{H}) ;{ }^{13} \mathrm{C} \mathrm{NMR}\left(125 \mathrm{MHz}, \mathrm{CDCl}_{3}\right) \delta 152.8,150.6,150.1,138.5,127.7,125.7$, 123.0, 76.8, 44.7, 34.6, 31.5; IR (neat) 3026, 2959, 2904, 2866, 2819, 2774, 1595, 1562, 1509, 1461, 1412, 1394, 1296, 1272, 1152, 1027, $1016 \mathrm{~cm}^{-1}$; HRMS m/z $269.2012\left[(\mathrm{M}+\mathrm{H})^{+}\right.$; calcd for $\mathrm{C}_{18} \mathrm{H}_{25} \mathrm{~N}_{2}$ : 269.2018].

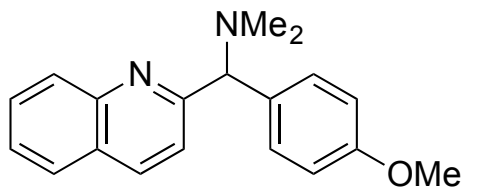

1-(4-Methoxyphenyl)- $N, N$-dimethyl-1-(quinolin-2-yl)methanamine (5i).

The product was prepared by General Procedure B using 1-(isoquinolin-3yl)- $N, N$-dimethylmethanamine 1i (37.3 mg, $0.20 \mathrm{mmol})$ and 4-bromoanisole $(44.9 \mathrm{mg}, 30.0 \mu \mathrm{L}, 0.24$ mmol) with $5 \mathrm{~mol} \% \mathrm{Pd}(\mathrm{OAc})_{2} / 7.5 \mathrm{~mol} \%$ NIXANTPHOS. The crude product was purified by flash 
column chromatography on silica gel (hexanes:EtOAc $=65: 35)$ to give the product $\mathbf{5 i}$ as a yellow oil (30.4 mg, 52\% yield). ${ }^{1} \mathrm{H} \mathrm{NMR}\left(500 \mathrm{MHz}, \mathrm{CDCl}_{3}\right) \delta 8.07(\mathrm{dd}, J=15.3,8.5 \mathrm{~Hz}, 2 \mathrm{H}), 7.70(\mathrm{dd}, J=16.3$, $8.3 \mathrm{~Hz}, 2 \mathrm{H}), 7.65(\mathrm{t}, J=7.7 \mathrm{~Hz}, 1 \mathrm{H}), 7.51(\mathrm{~d}, J=8.7 \mathrm{~Hz}, 2 \mathrm{H}), 7.46(\mathrm{t}, J=7.5 \mathrm{~Hz}, 1 \mathrm{H}), 6.82(\mathrm{~d}, J=8.7$ $\mathrm{Hz}, 2 \mathrm{H}), 4.41(\mathrm{~s}, 1 \mathrm{H}), 3.73(\mathrm{~s}, 3 \mathrm{H}), 2.26(\mathrm{~s}, 6 \mathrm{H}) ;{ }^{13} \mathrm{C} \mathrm{NMR}\left(125 \mathrm{MHz}, \mathrm{CDCl}_{3}\right) \delta 163.5,159.0,147.8$, $136.9,134.0,129.5,129.4,127.6,127.6,126.3,120.2,114.1,79.7,55.4,44.9$. IR (neat) 3060, 2950, 2932, 2863, 2821, 2775, 1608, 1599, 1563, 1510, 1462, 1440, 1426, 1303, 1291, 1248, 1178, 1152 , 1115, $1031 \mathrm{~cm}^{-1}$; HRMS m/z 293.1646 [(M+H) ${ }^{+}$; calcd for $\left.\mathrm{C}_{19} \mathrm{H}_{21} \mathrm{~N}_{2} \mathrm{O}: 293.1654\right]$.

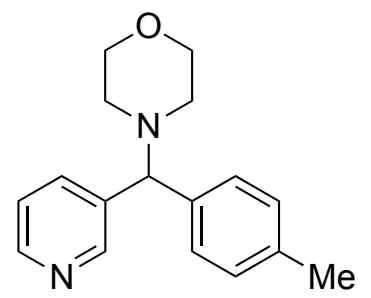

4-(Pyridin-3-yl(p-tolyl)methyl)morpholine (5j). The product was prepared by General Procedure B $\left(\mathrm{Pd}(\mathrm{OAc})_{2} / \mathrm{NIXANTPHOS}\right.$ stock solution was prepared in THF) using 4-(pyridin-3-ylmethyl)morpholine 1j (35.6 mg, $0.20 \mathrm{mmol})$, 4bromotoluene $(41.0 \mathrm{mg}, 29.5 \mu \mathrm{L}, 0.24 \mathrm{mmol})$ and $\mathrm{KN}\left(\mathrm{SiMe}_{3}\right)_{2}(59.8 \mathrm{mg}, 0.30$ mmol) with $5 \mathrm{~mol} \% \mathrm{Pd}(\mathrm{OAc})_{2} / 7.5 \mathrm{~mol} \%$ NIXANTPHOS. The crude product was purified by flash column chromatography on silica gel hexanes:EtOAc $=60: 40)$ to give the product $\mathbf{5} \mathbf{j}$ as a colourless oil (34.9 mg, 65\% yield). ${ }^{1} \mathrm{H}$ NMR $\left(500 \mathrm{MHz}, \mathrm{CDCl}_{3}\right) \delta 8.66(\mathrm{~d}, J=1.3 \mathrm{~Hz}, 1 \mathrm{H}), 8.43(\mathrm{~d}, J=3.6 \mathrm{~Hz}, 1 \mathrm{H})$, $7.74(\mathrm{dt}, J=7.9,1.7 \mathrm{~Hz}, 1 \mathrm{H}), 7.28(\mathrm{~d}, J=8.1 \mathrm{~Hz}, 2 \mathrm{H}), 7.20(\mathrm{dd}, J=7.9,4.8 \mathrm{~Hz}, 1 \mathrm{H}), 7.10(\mathrm{~d}, J=7.8$ $\mathrm{Hz}, 2 \mathrm{H}), 4.22(\mathrm{~s}, 1 \mathrm{H}), 3.71(\mathrm{t}, J=4.7 \mathrm{~Hz}, 4 \mathrm{H}), 2.41-2.33(\mathrm{~m}, 4 \mathrm{H}), 2.29(\mathrm{~s}, 3 \mathrm{H}) ;{ }^{13} \mathrm{C} \mathrm{NMR}(125 \mathrm{MHz}$ $\left.\mathrm{CDCl}_{3}\right) \delta 149.7,148.7,138.3,138.3,137.4,135.5,129.7,128.0,123.8,74.1,67.3,52.7,21.2$. IR (neat) $3028,2958,2920,2854,2808,1661,1606,1586,1576,1511,1453,1426,1307,1281,1252,1179$, 1117, 1070, 1026, $1009 \mathrm{~cm}^{-1}$; HRMS m/z 269.1652 [(M+H) ${ }^{+}$; calcd for $\left.\mathrm{C}_{17} \mathrm{H}_{21} \mathrm{~N}_{2} \mathrm{O}: 269.1654\right]$. 


\section{References}

1 A. R. Katritzky, J. B. Bapat, R. J. Blade, B. P. Leddy, P.-L. Nie, C. A. Ramsden and S. S. Thind, J. Chem. Soc., Perkin Trans. 1, 1979, 418-425.

2 E. B. Sanders, H. V. Secor and J. I. Seeman, J. Org. Chem., 1978, 43, 324-330.

3 G. B. Boursalian, M.-Y. Ngai, K. N. Hojczyk and T. Ritter, J. Am. Chem. Soc., 2013, 135, 13278-13281.

4 S. S. Massoud, A. E. Guilbeau, H. T. Luong, R. Vicente, J. H. Albering, R. C. Fischer and F. A. Mautner, Polyhedron, 2013, 54, 26-33.

$5 \quad$ F. Wang and L. M. Sayre, J. Am. Chem. Soc., 1992, 114, 248-255.

6 Y.-C. Lin, K.-H. Yu, Y.-F. Lin, G.-H. Lee, Y. Wang, S.-T. Liu and J.-T. Chen, Dalton Trans., 2012, 41, 6661-6670.

7 Y. Hu, L. Liang, W.-t. Wei, X. Sun, X.-j. Zhang and M. Yan, Tetrahedron, 2015, 71, 1425-1430.

8 Q. Li, C. W. Liskey and J. F. Hartwig, J. Am. Chem. Soc., 2014, 136, 8755-8765.

9 R. Frauenlob, C. García, G. A. Bradshaw, H. M. Burke and E. Bergin, J. Org. Chem., 2012, 77, 4445-4449. 
${ }^{1} \mathrm{H}$ NMR (500 MHz) spectrum of $\mathbf{1 a}$.

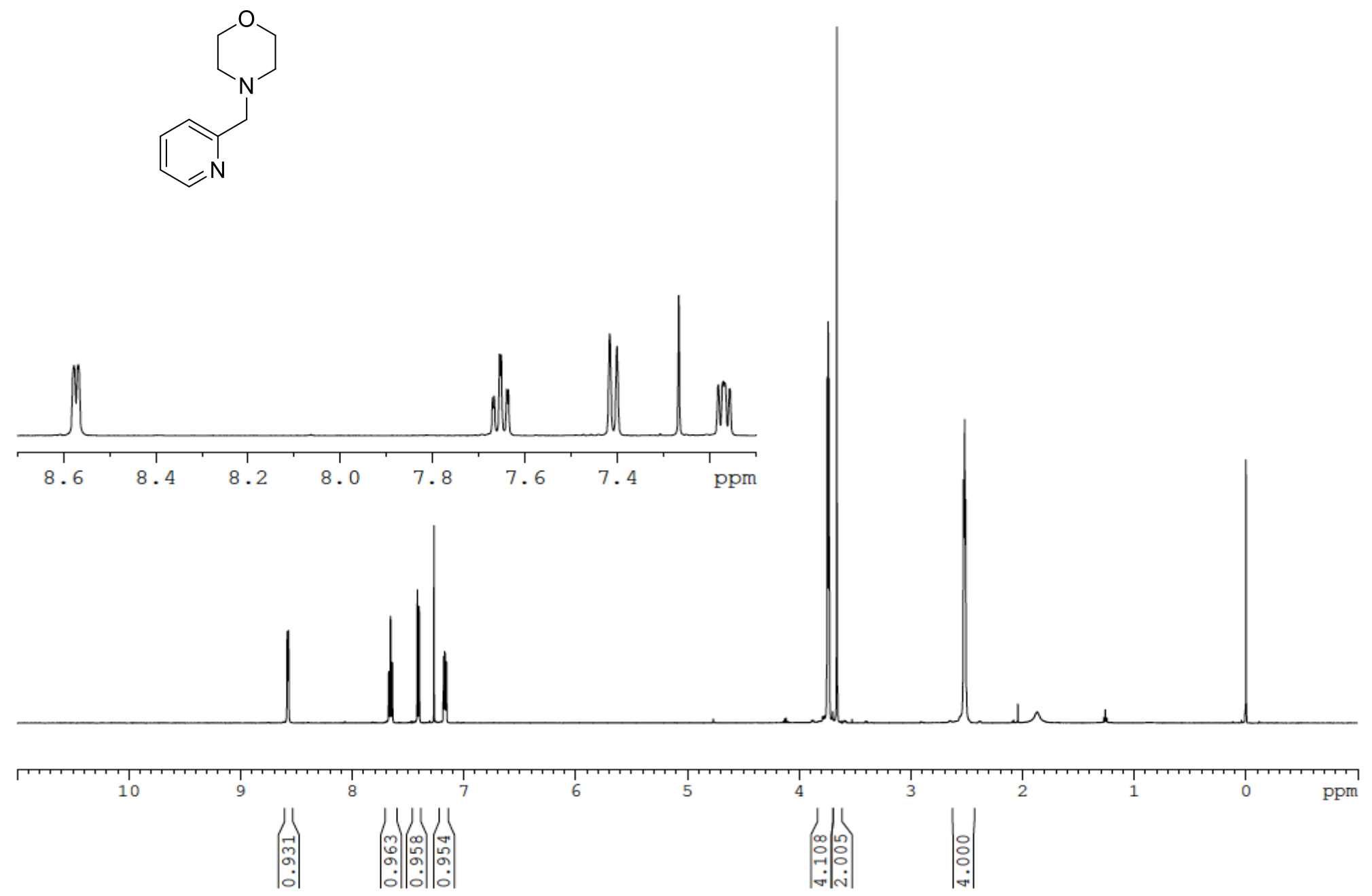


${ }^{13} \mathrm{C}$ NMR (125 MHz) spectrum of $\mathbf{1 a}$.
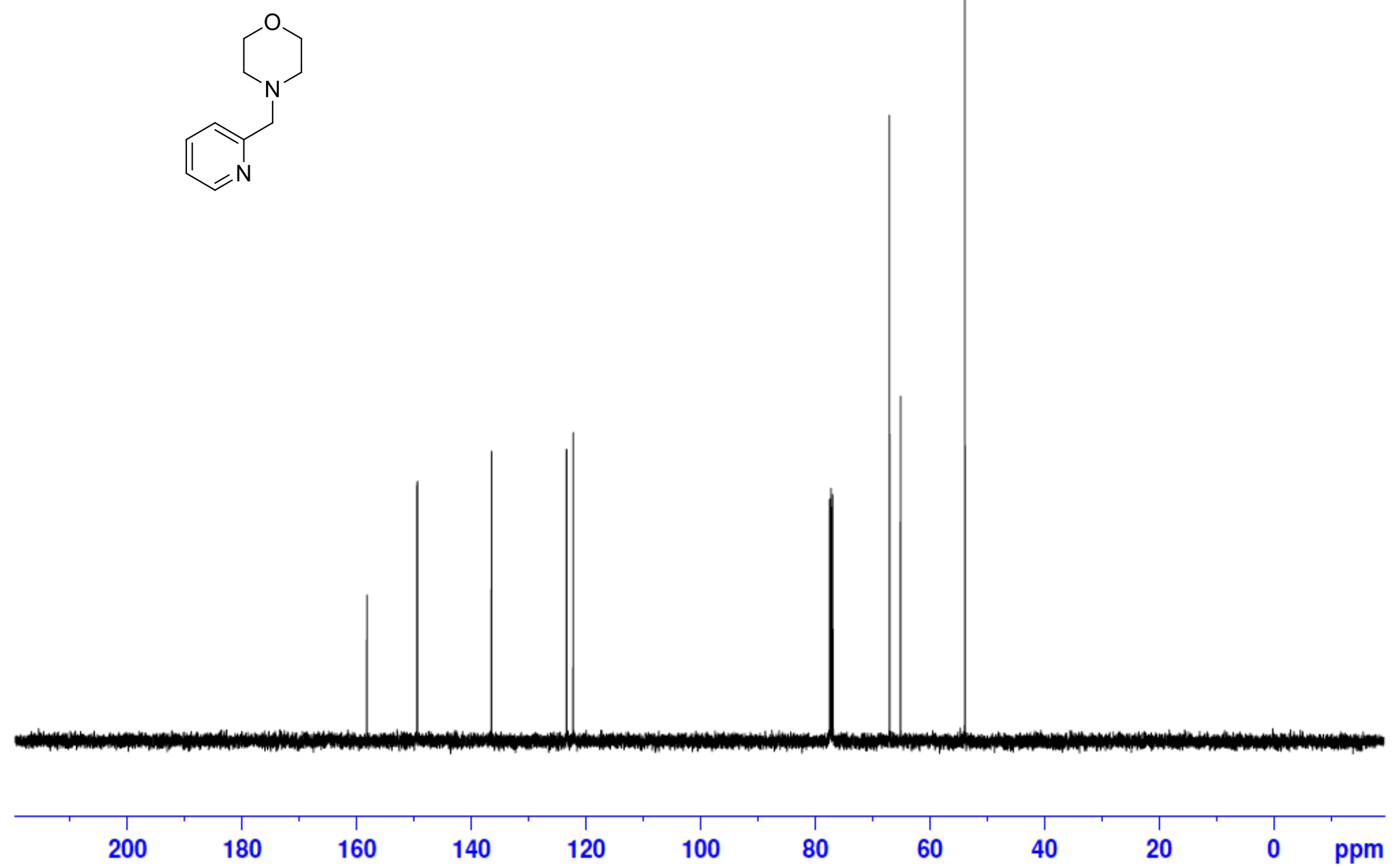
${ }^{1} \mathrm{H}$ NMR (500 MHz) spectrum of $\mathbf{1 b}$.

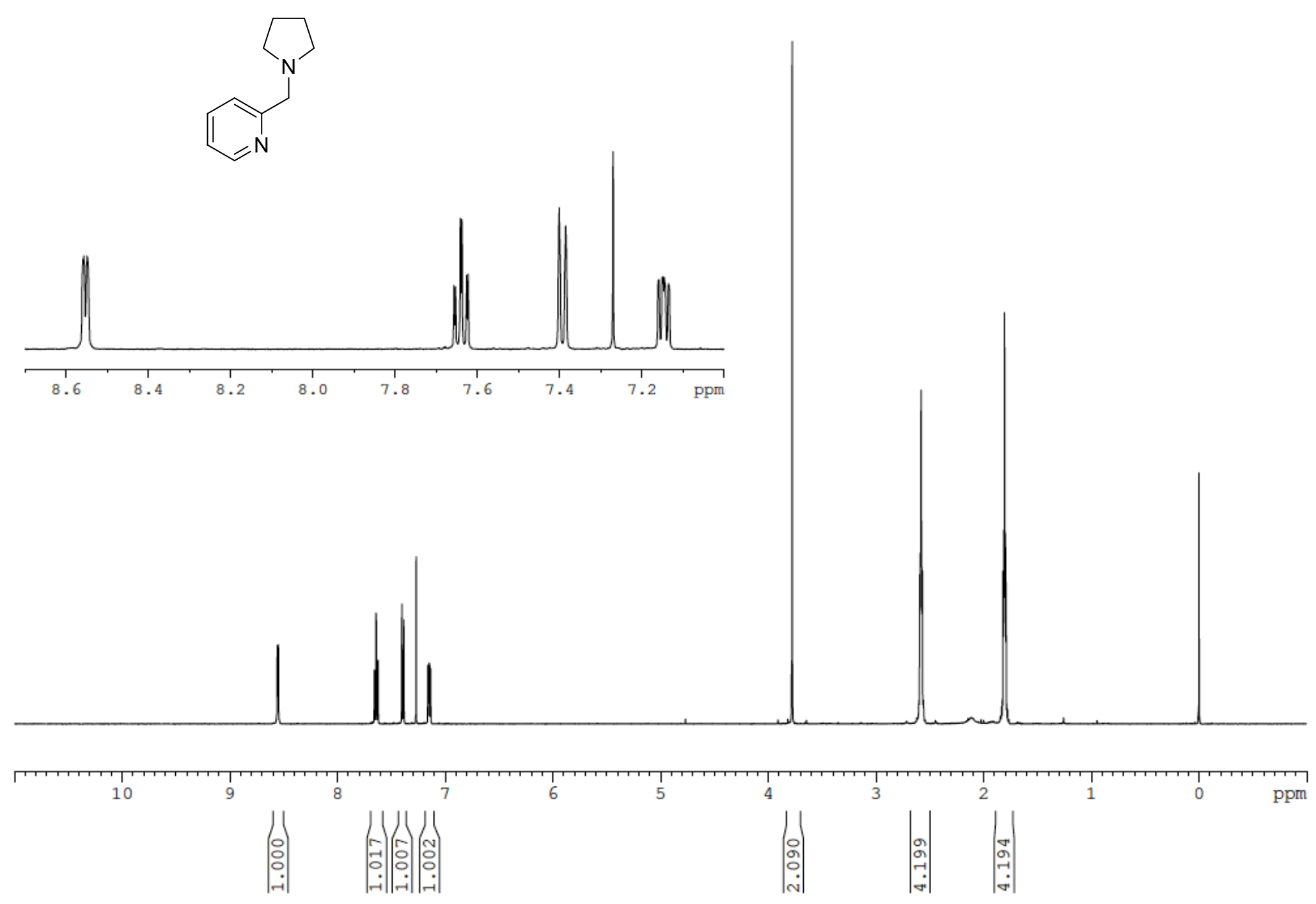

S 33 
${ }^{13} \mathrm{C}$ NMR (125 MHz) spectrum of $\mathbf{1 b}$.
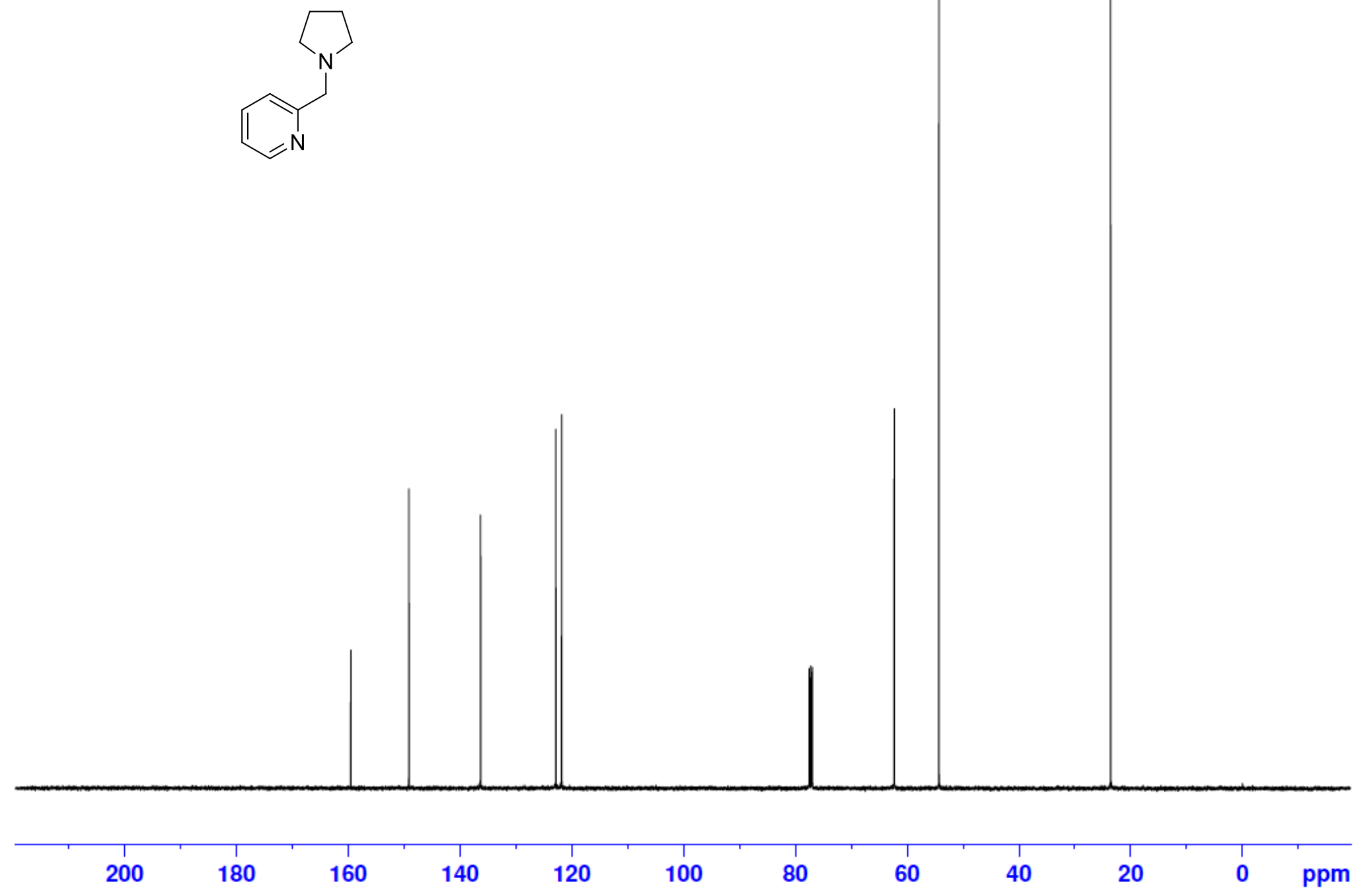
${ }^{1} \mathrm{H}$ NMR (500 MHz) spectrum of $\mathbf{1 c .}$

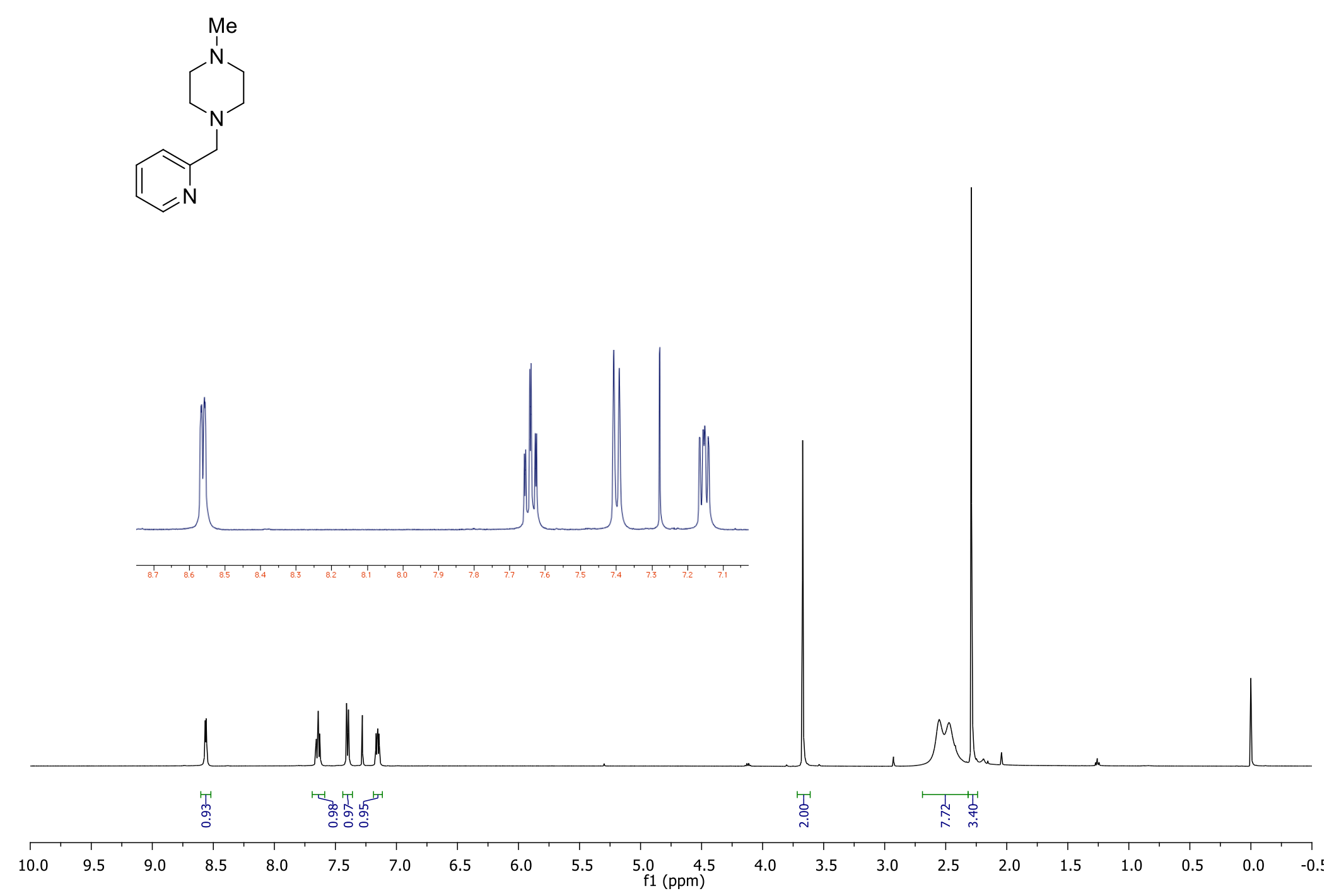


${ }^{13} \mathrm{C}$ NMR (125 MHz) spectrum of 1c.
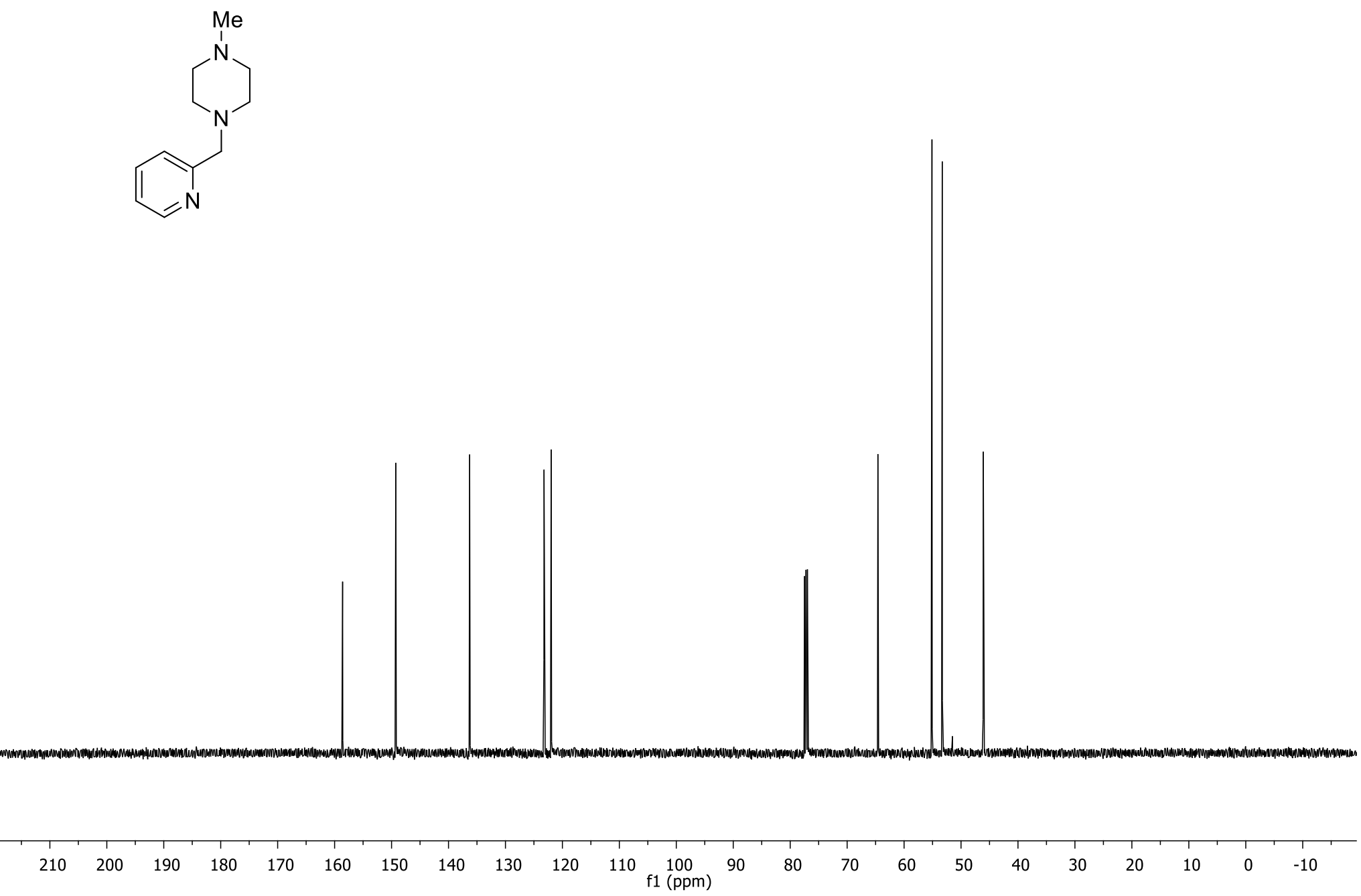
${ }^{1} \mathrm{H}$ NMR (500 MHz) spectrum of $\mathbf{1 d .}$

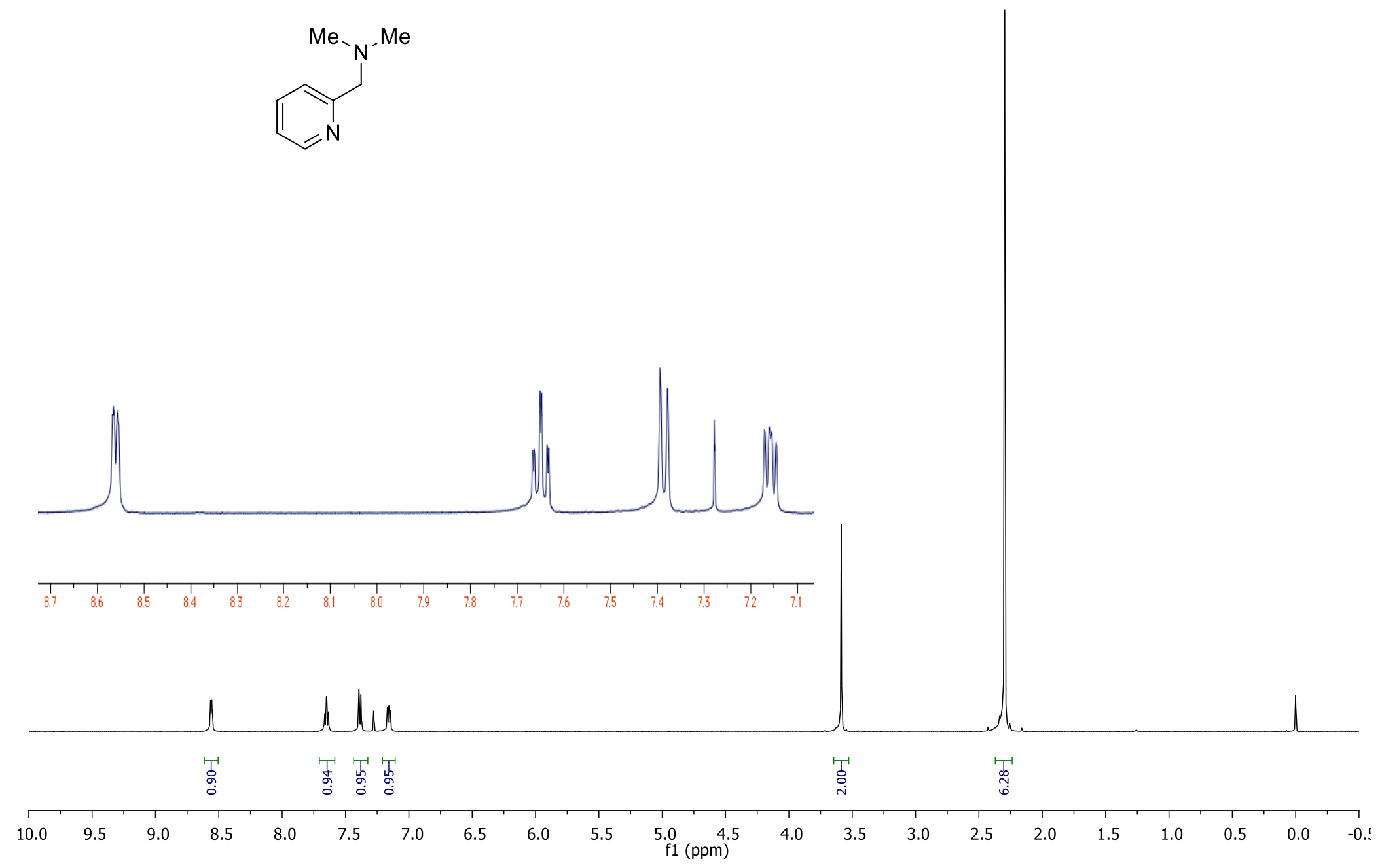


${ }^{13} \mathrm{C}$ NMR (125 MHz) spectrum of $\mathbf{1 d}$.
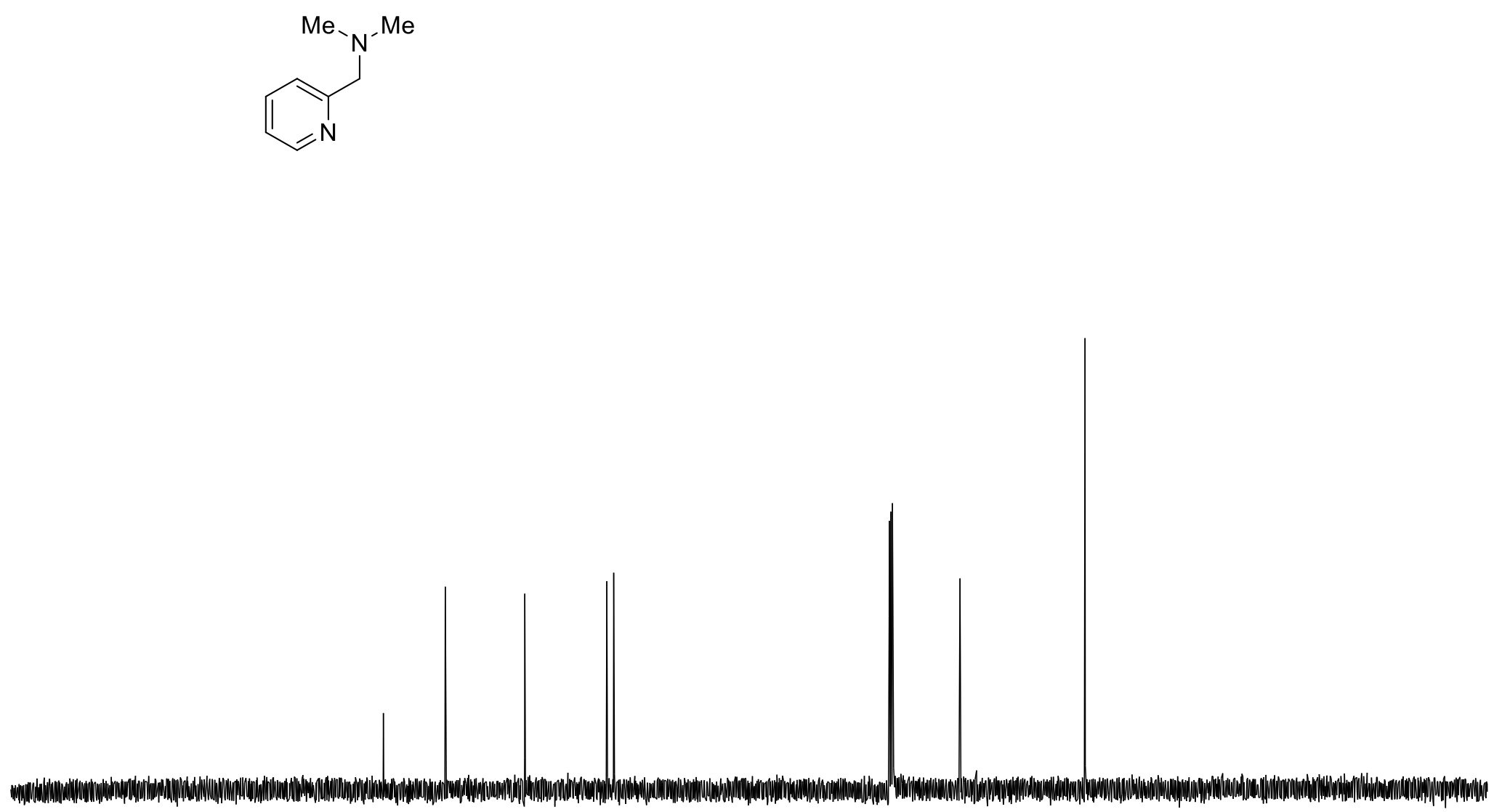
${ }^{1} \mathrm{H}$ NMR (500 MHz) spectrum of $\mathbf{1 e .}$

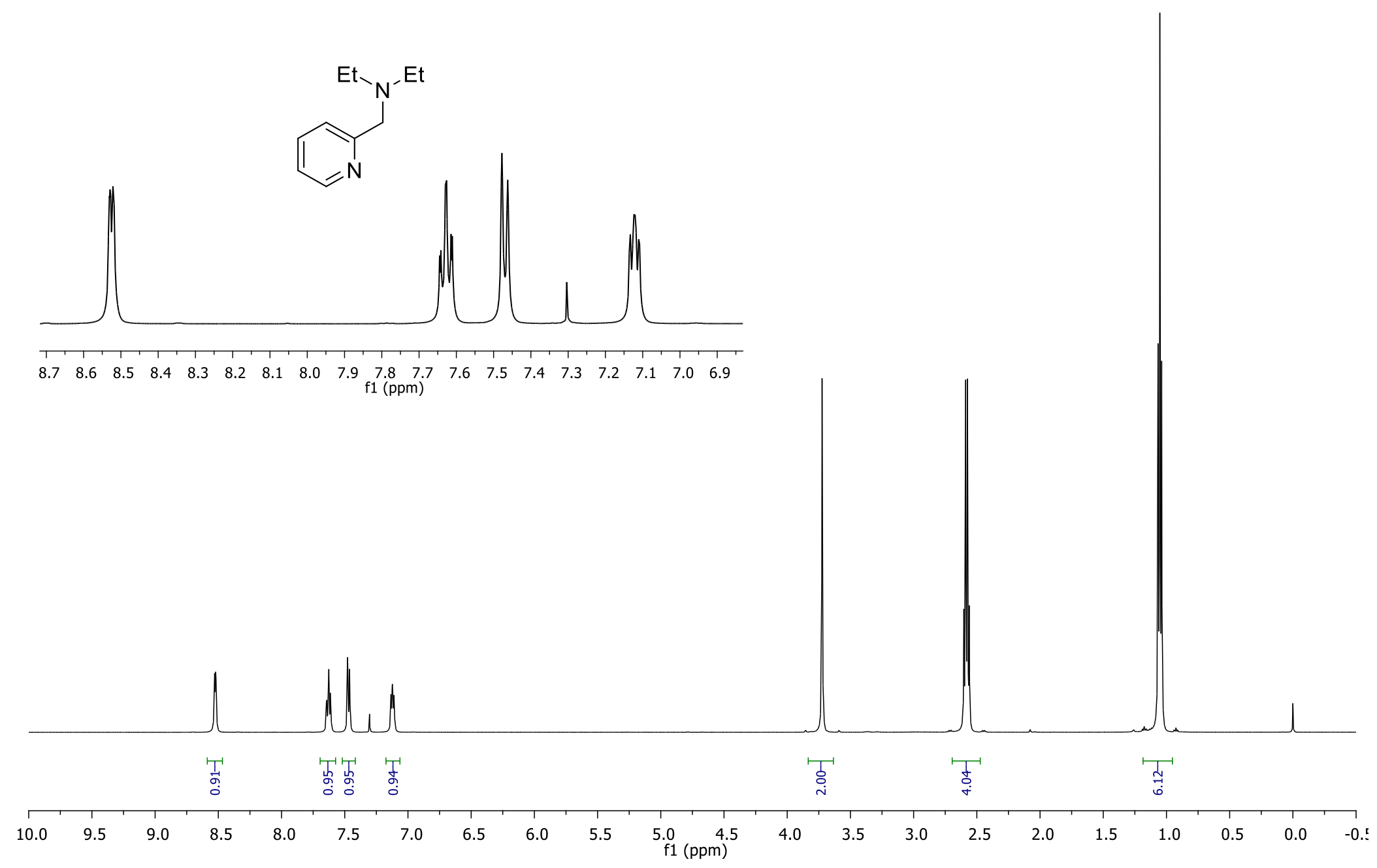


${ }^{13} \mathrm{C}$ NMR (125 MHz) spectrum of $\mathbf{1 e .}$
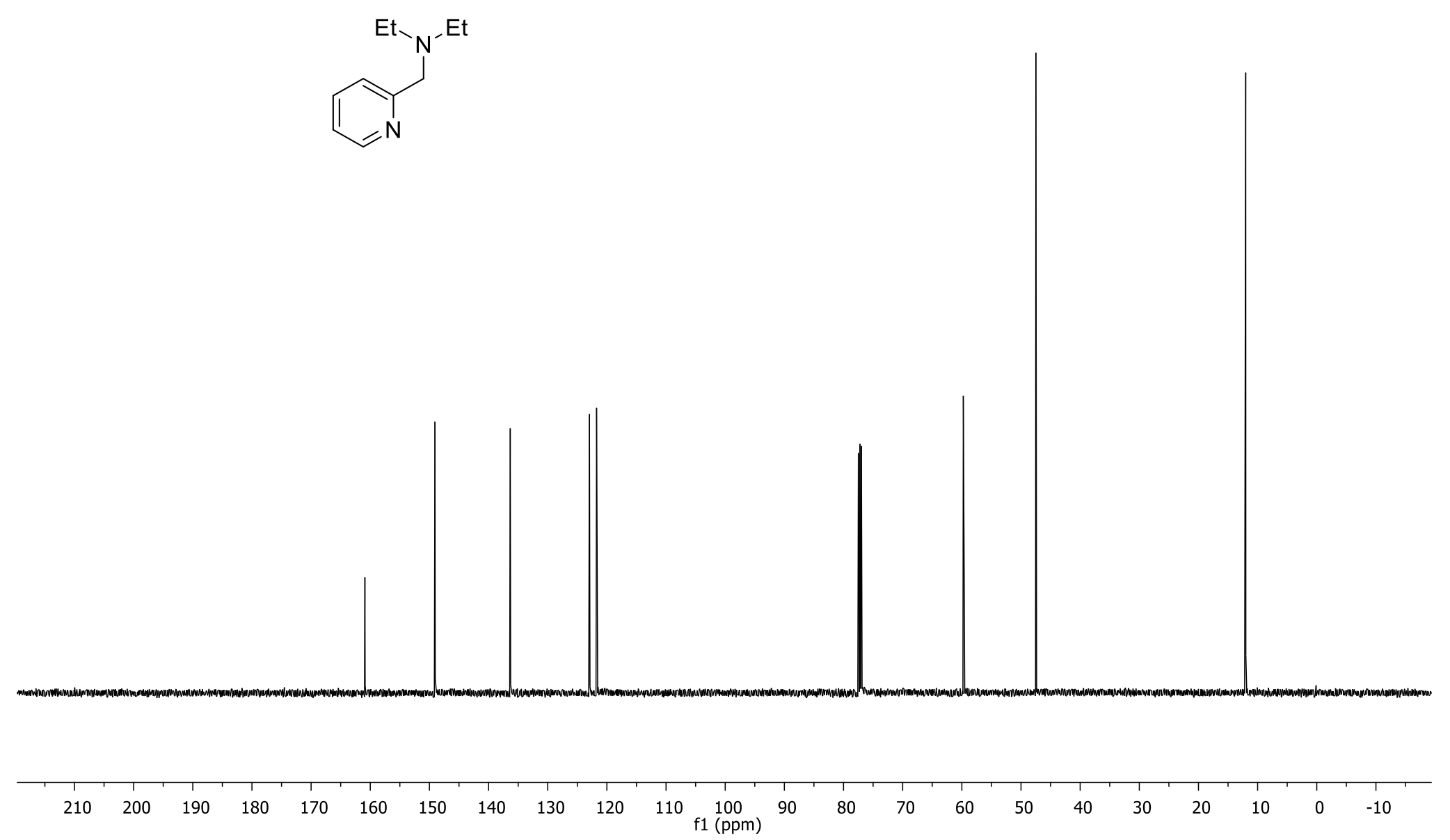

S 40 
${ }^{1} \mathrm{H}$ NMR (500 MHz) spectrum of $\mathbf{1 f .}$

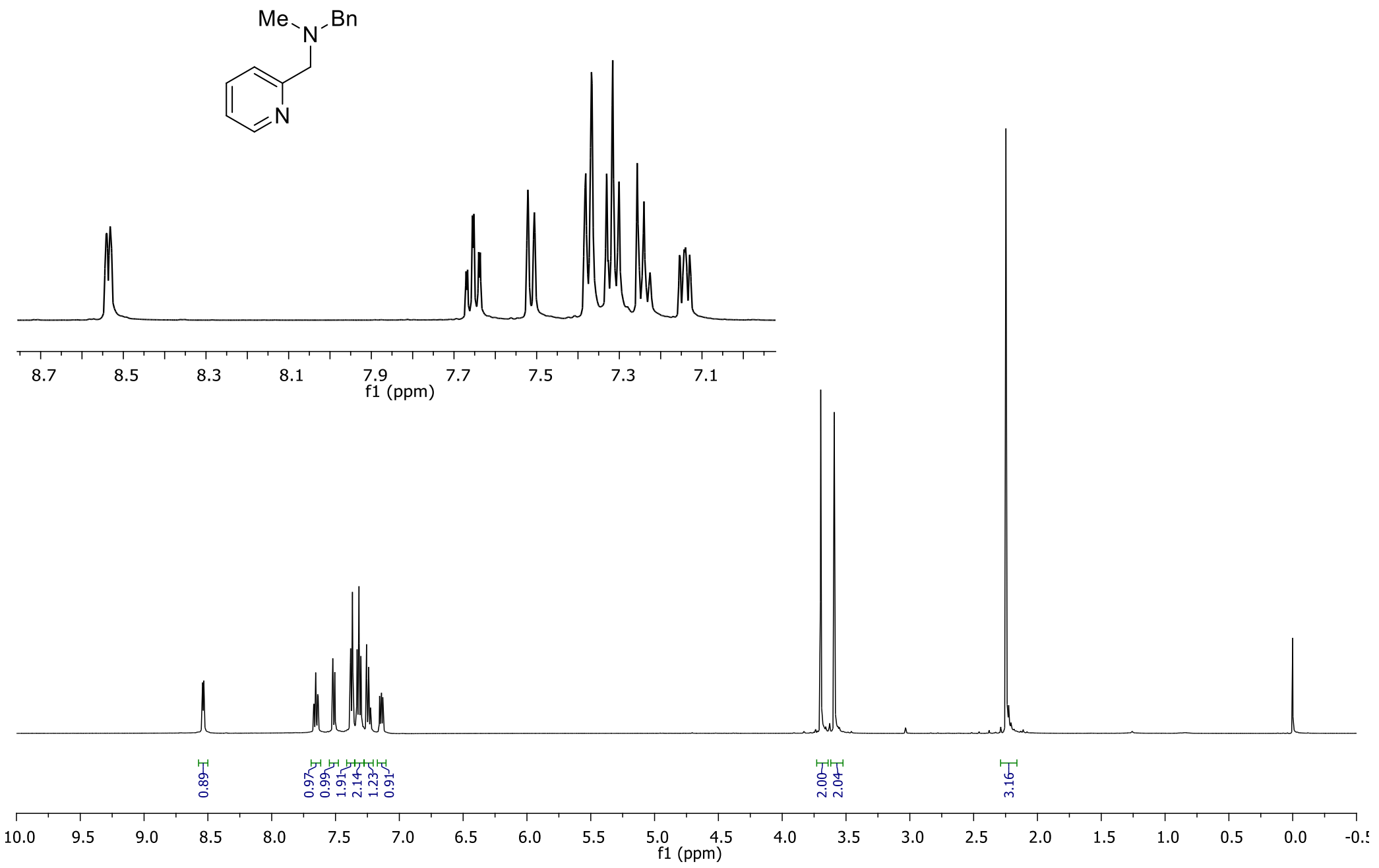


${ }^{13} \mathrm{C}$ NMR (125 MHz) spectrum of $\mathbf{1 f}$.

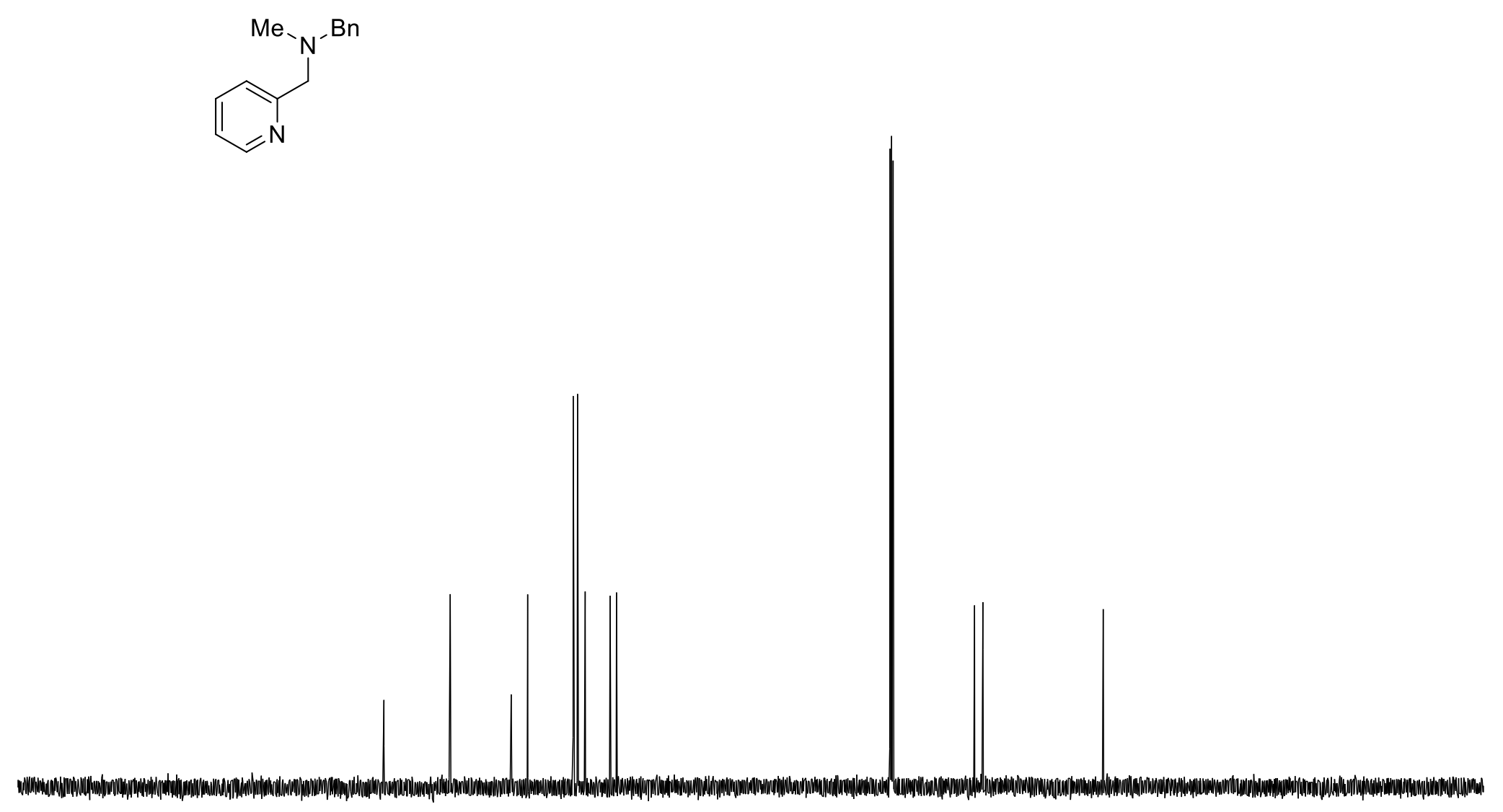


${ }^{1} \mathrm{H}$ NMR (500 MHz) spectrum of $\mathbf{1 g}$.

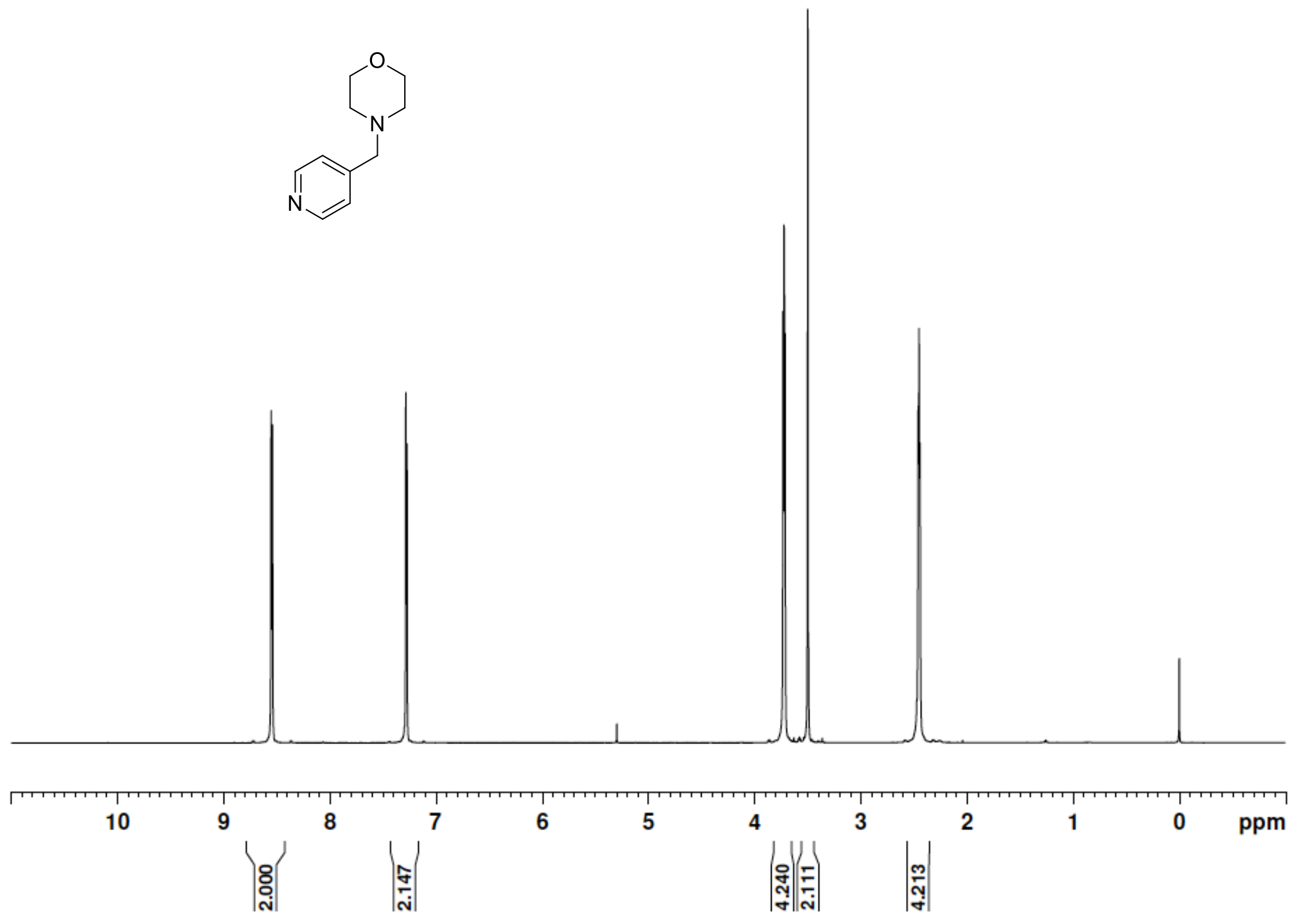


${ }^{13} \mathrm{C}$ NMR (125 MHz) spectrum of $\mathbf{1 g}$.

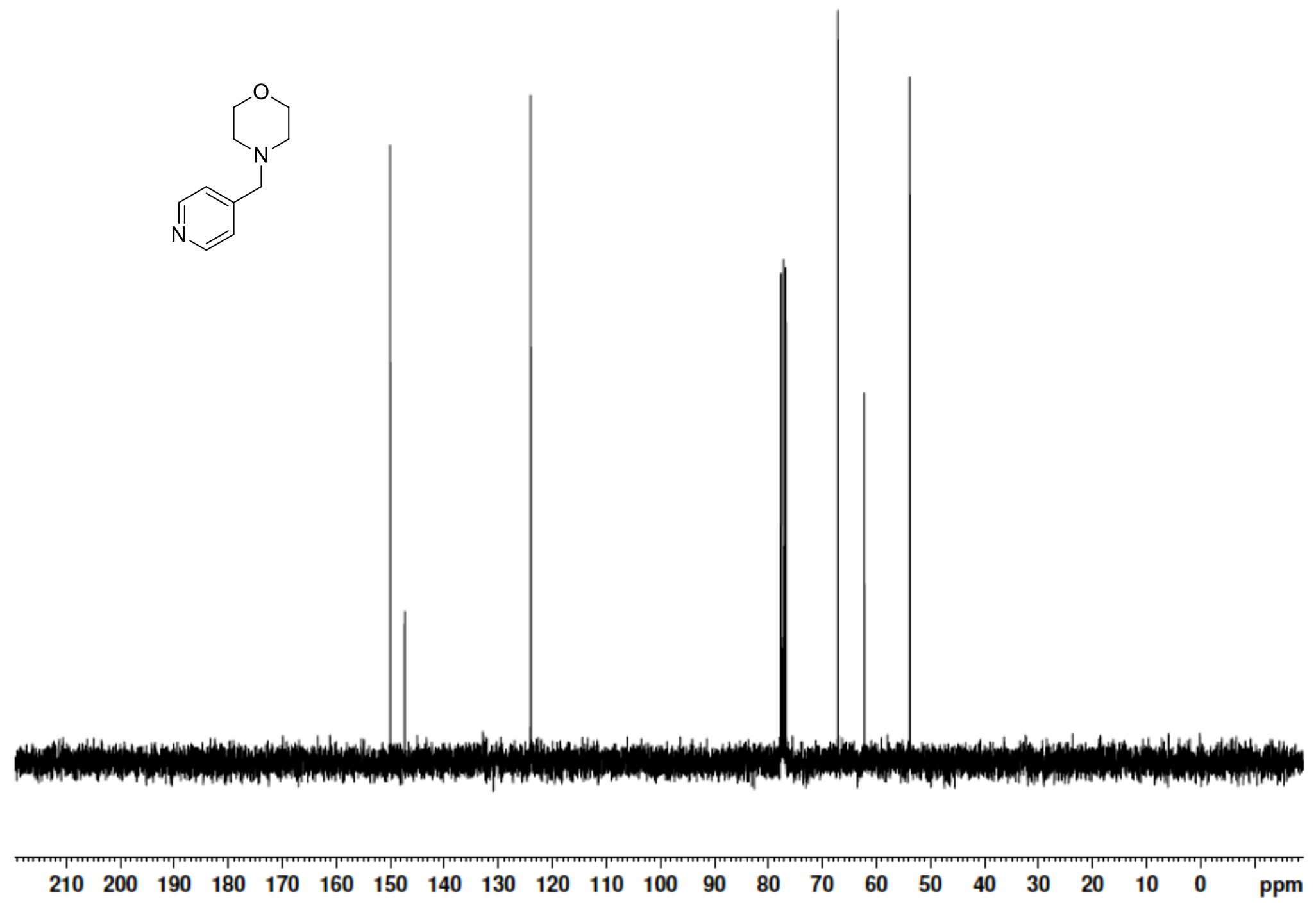


${ }^{1} \mathrm{H}$ NMR (500 MHz) spectrum of $\mathbf{1} \mathbf{h}$.

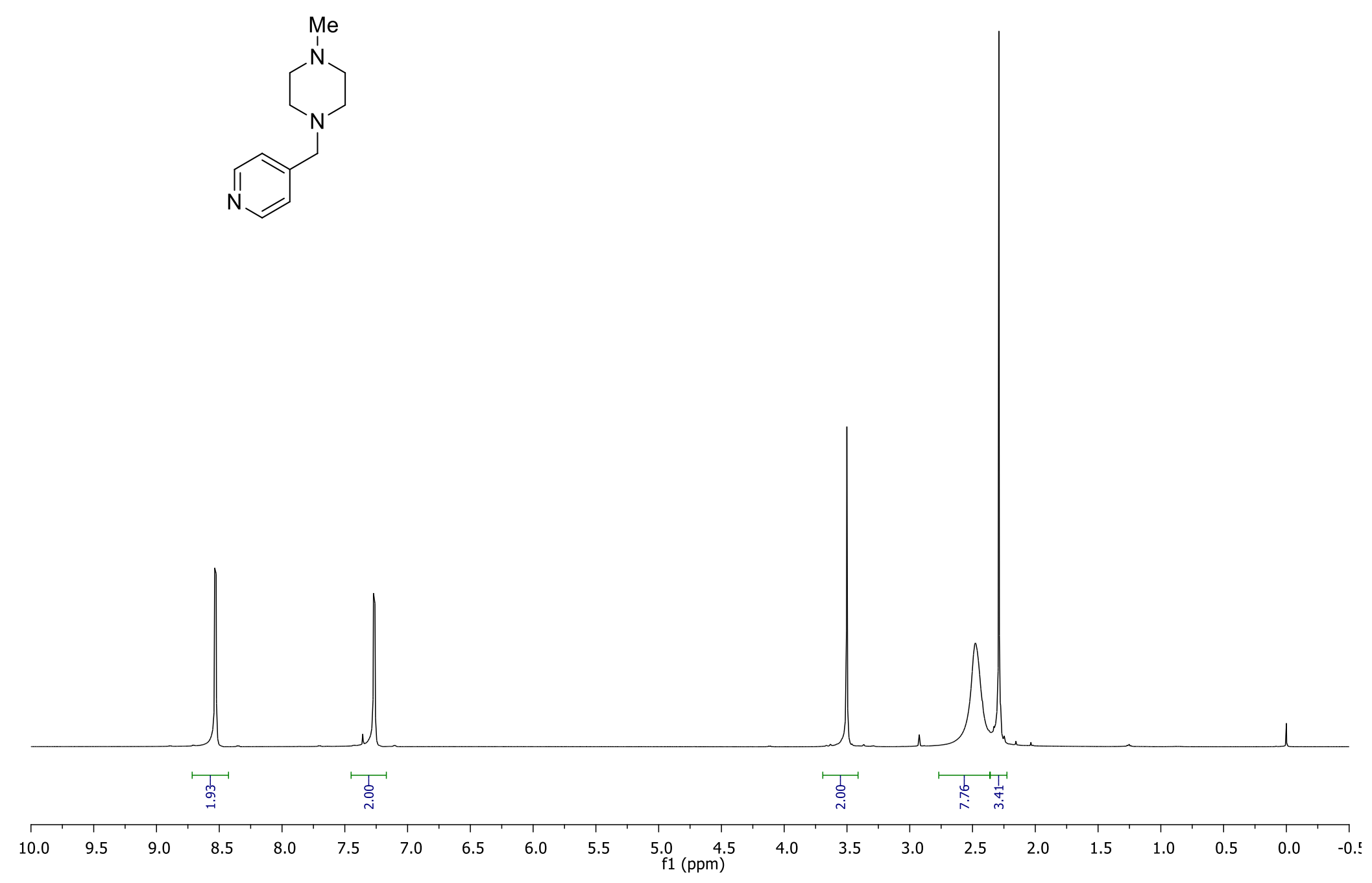


${ }^{13} \mathrm{C}$ NMR (125 MHz) spectrum of $\mathbf{1 h}$.

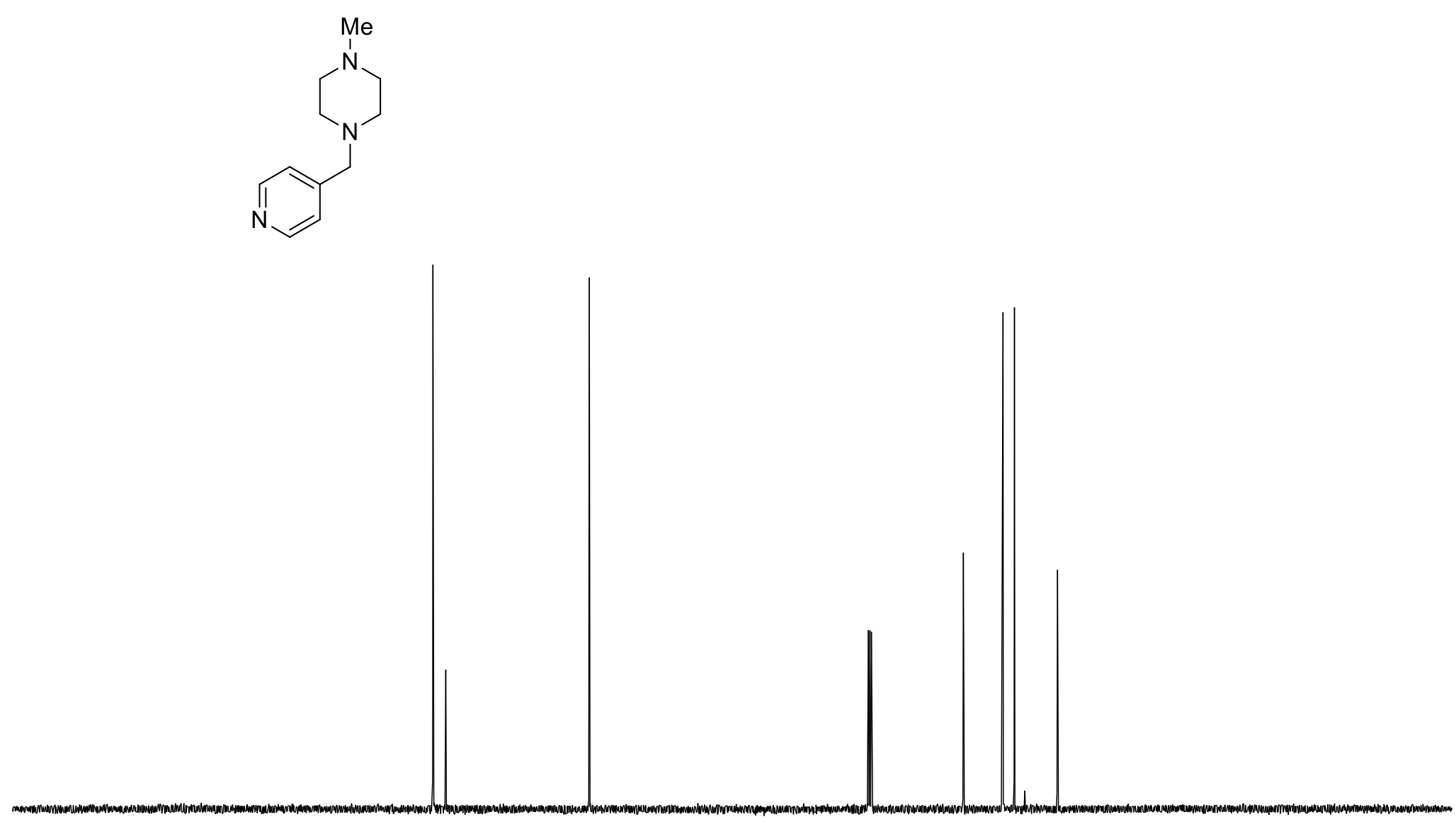


${ }^{1} \mathrm{H}$ NMR (500 MHz) spectrum of $\mathbf{1 i}$.

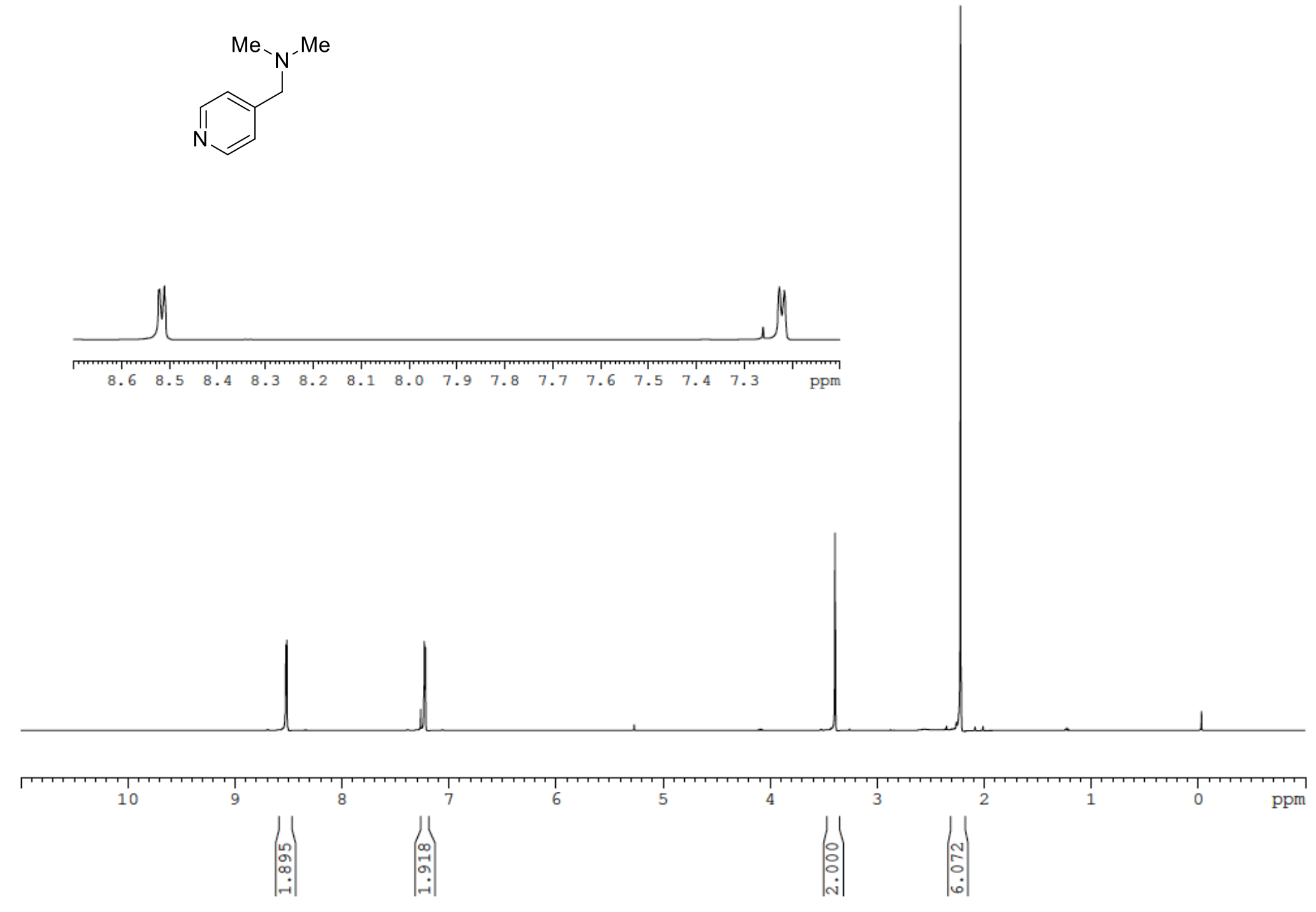


${ }^{13} \mathrm{C}$ NMR (125 MHz) spectrum of $\mathbf{1 i}$.

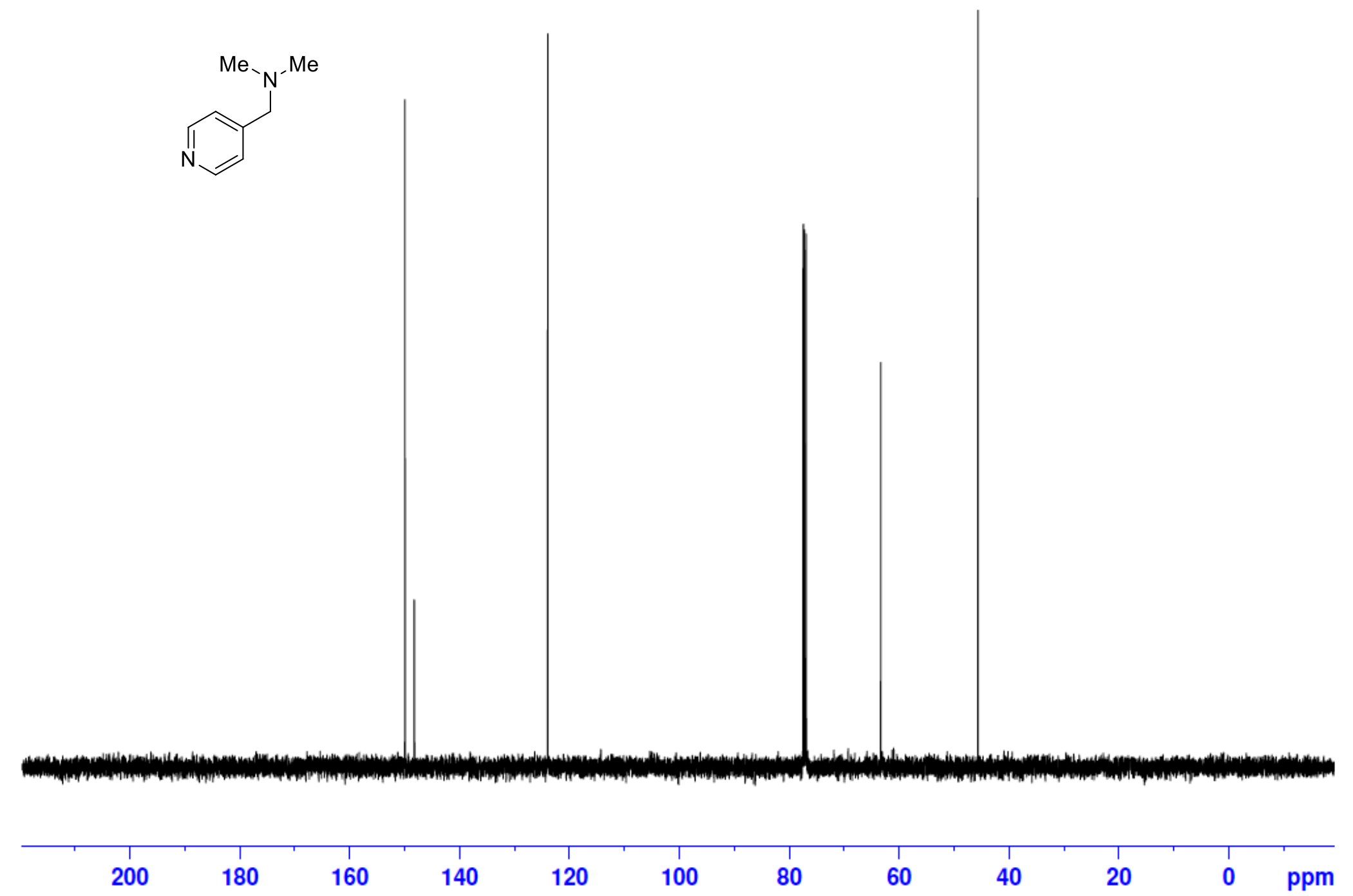

S 48 
${ }^{1} \mathrm{H}$ NMR (500 MHz) spectrum of $\mathbf{1 j}$.
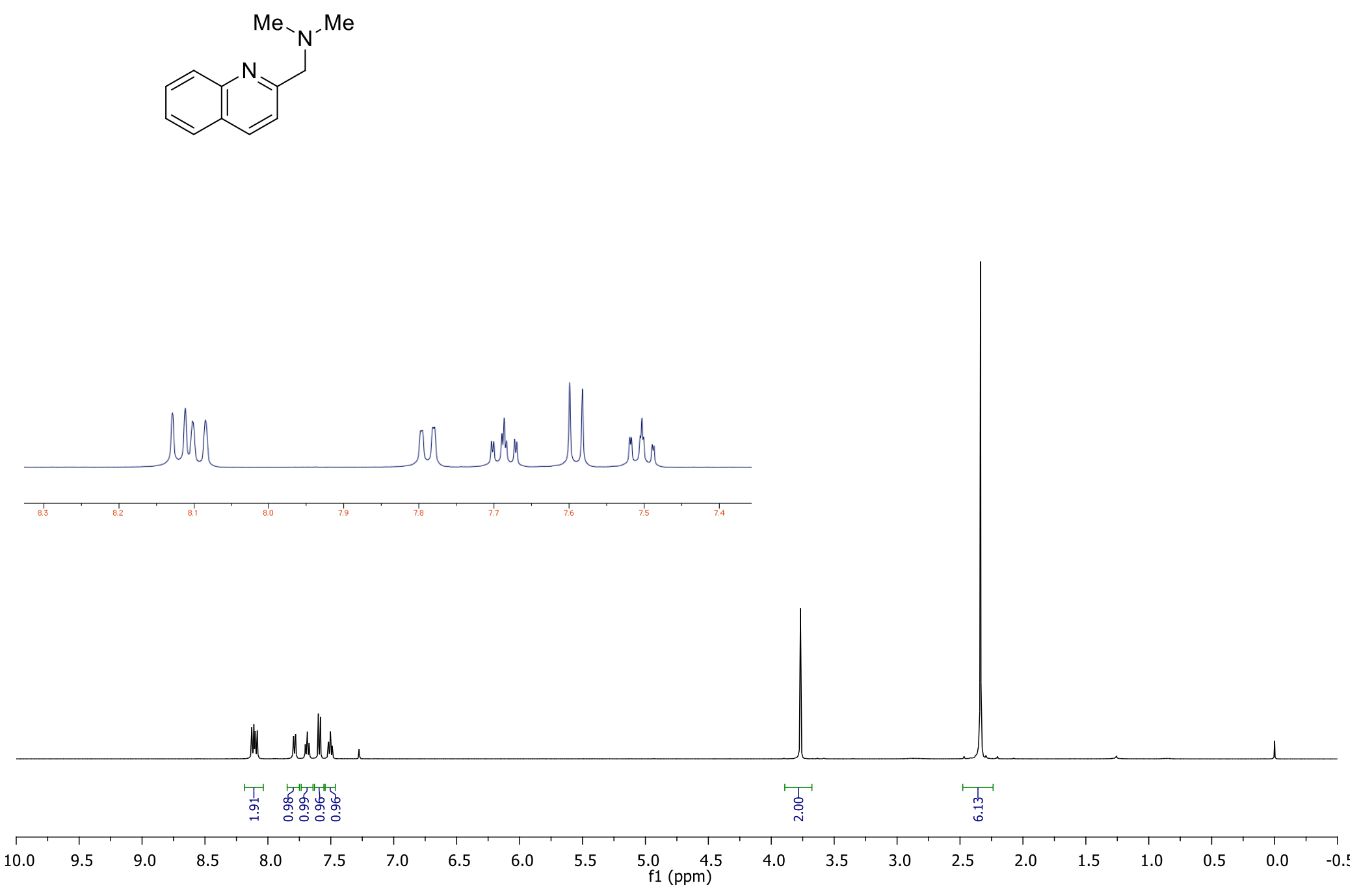
${ }^{13} \mathrm{C}$ NMR (125 MHz) spectrum of $\mathbf{1 j}$.

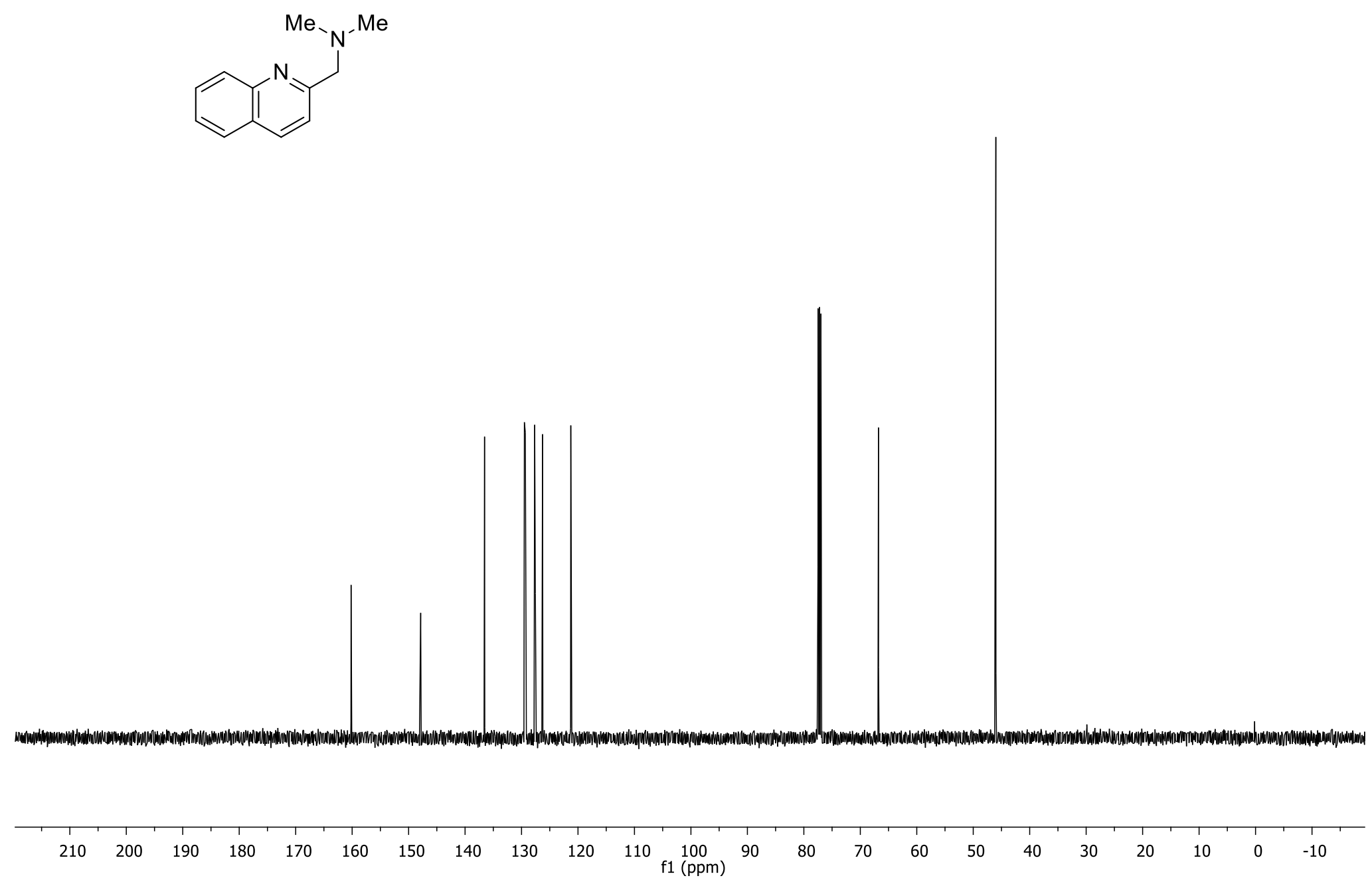


${ }^{1} \mathrm{H}$ NMR (500 MHz) spectrum of $\mathbf{1 k}$.

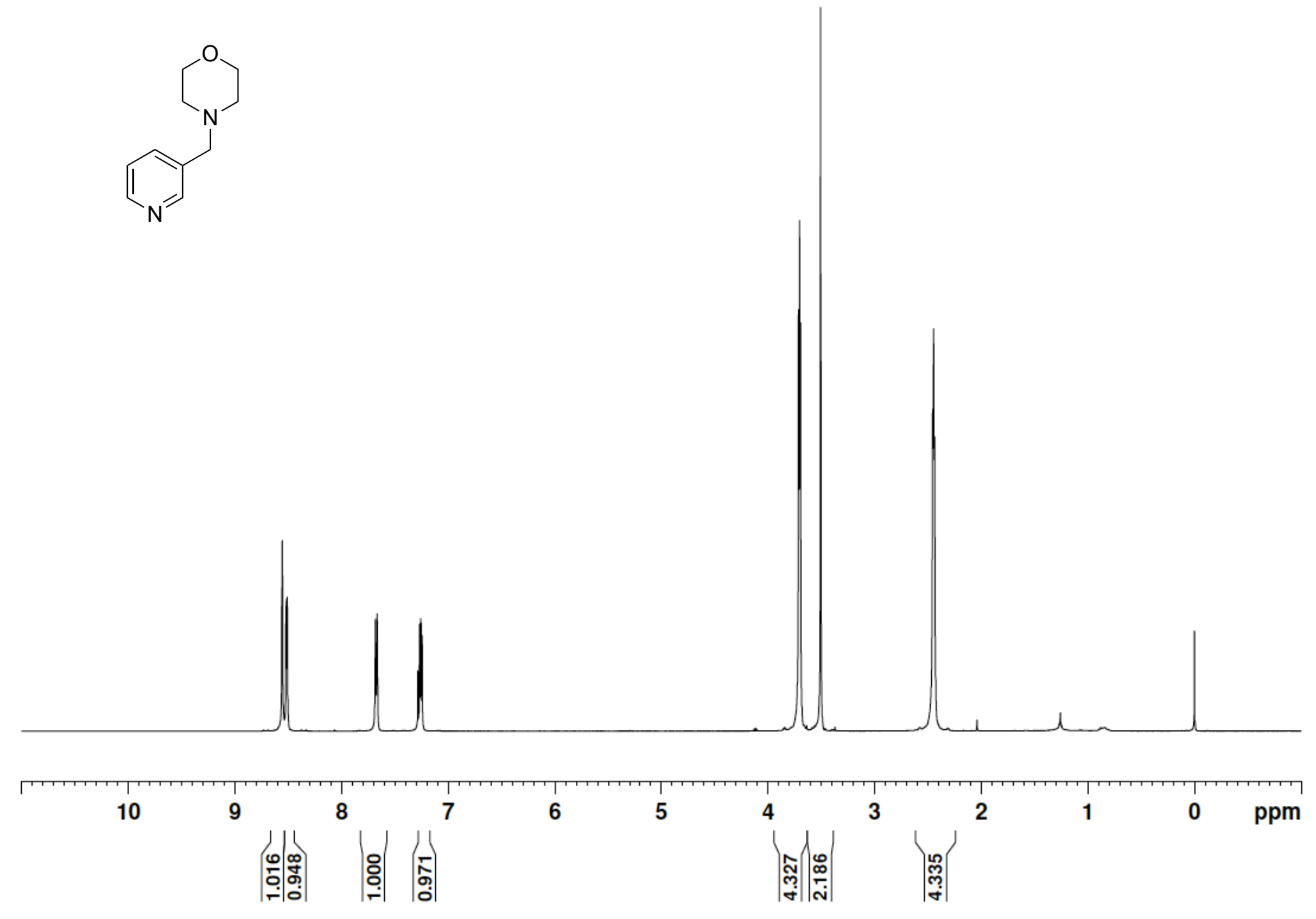


${ }^{13} \mathrm{C}$ NMR (125 MHz) spectrum of $\mathbf{1} \mathbf{k}$.

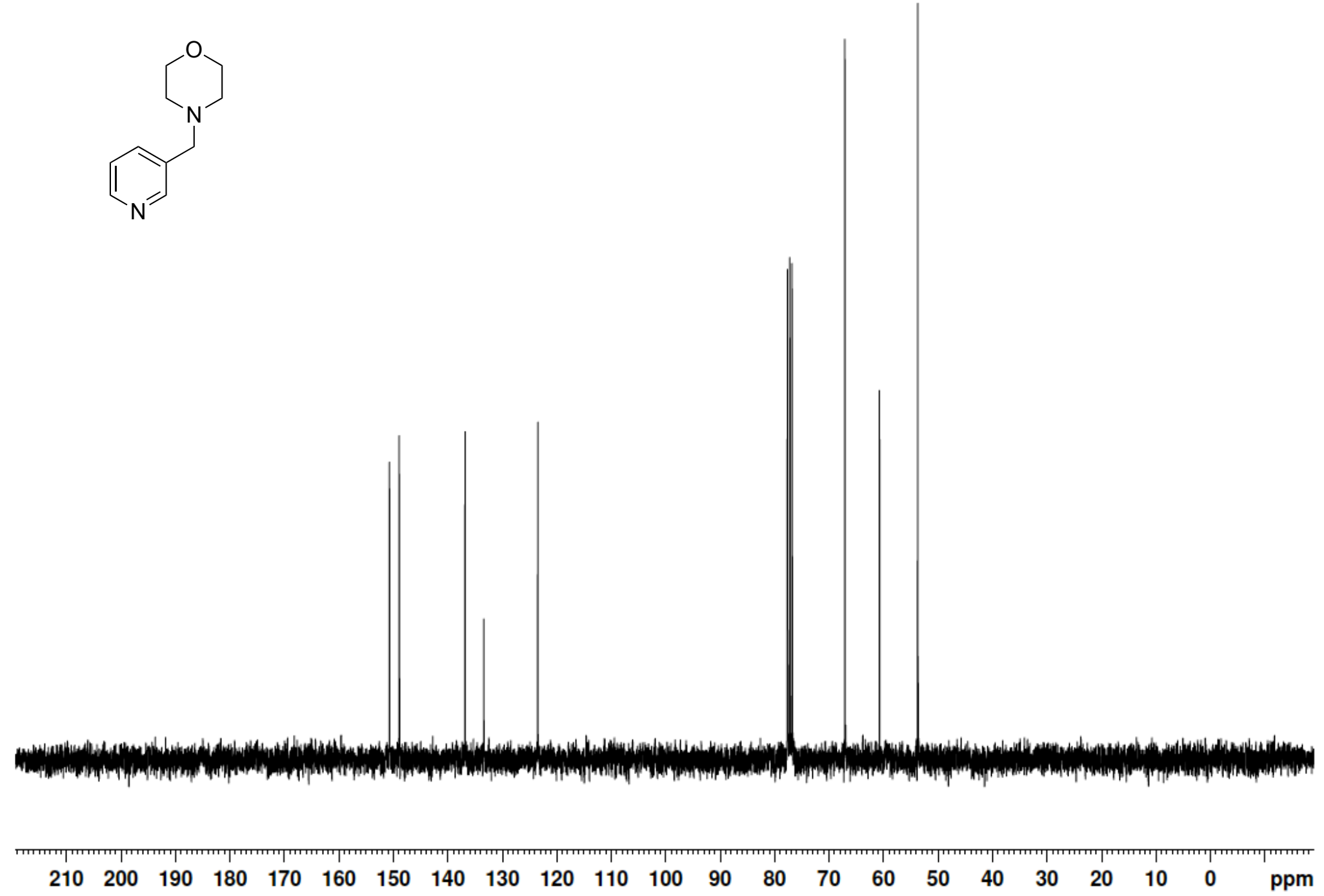


${ }^{1} \mathrm{H}$ NMR (500 MHz) spectrum of $\mathbf{3 a}$.
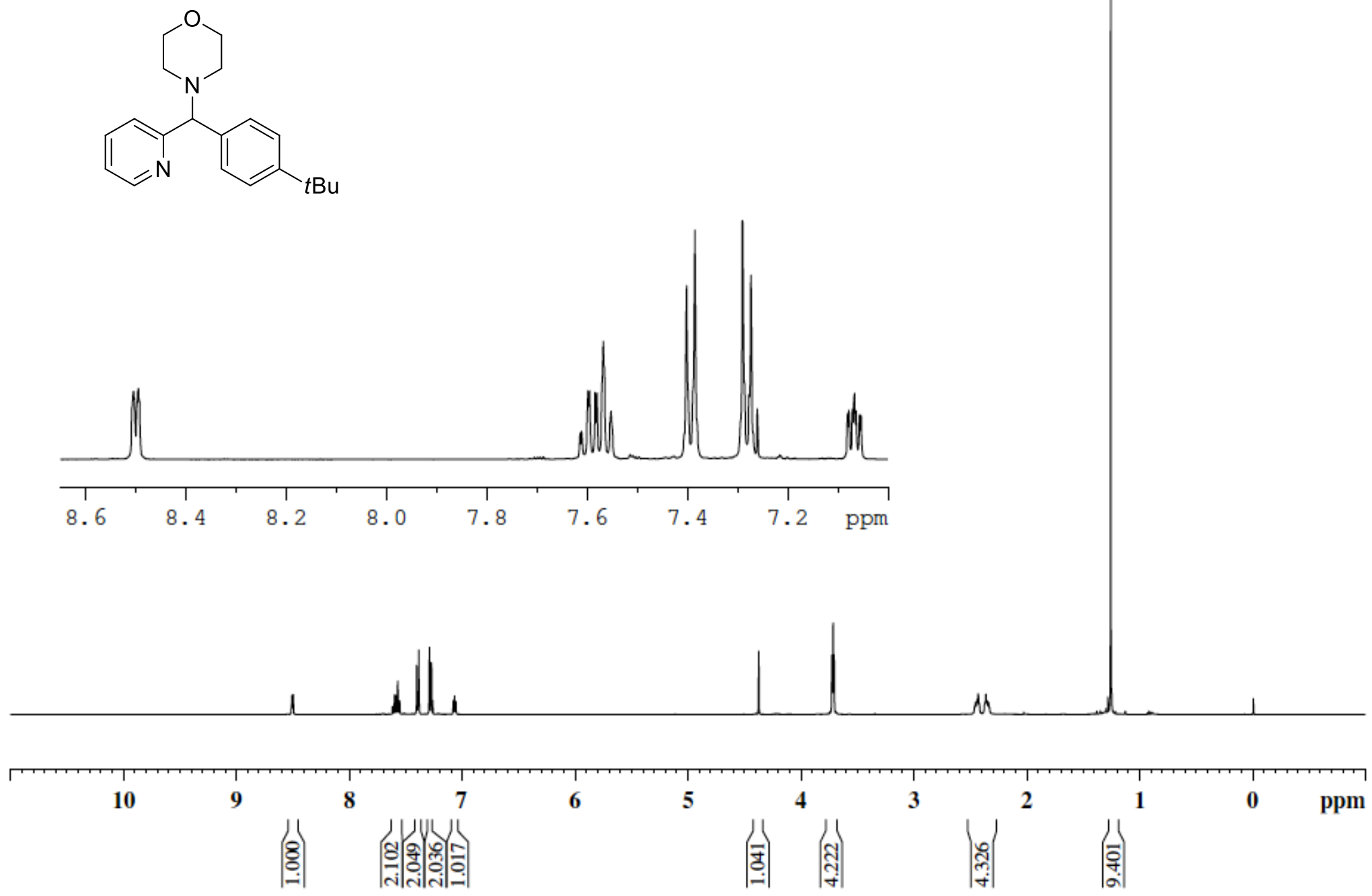

S 53 
${ }^{13} \mathrm{C}$ NMR (125 MHz) spectrum of $\mathbf{3 a}$.

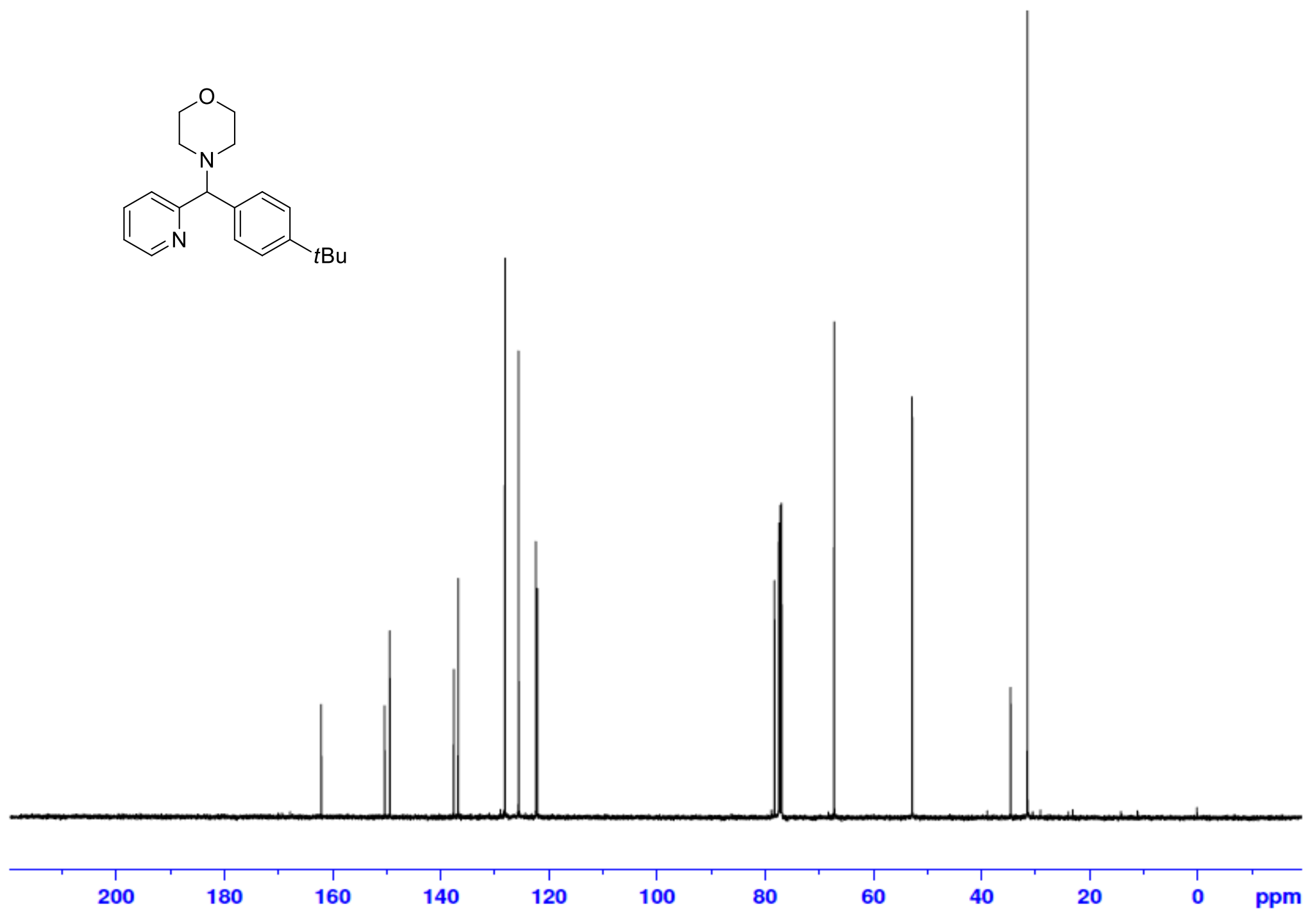


${ }^{1} \mathrm{H}$ NMR (500 MHz) spectrum of $\mathbf{3 b}$.<smiles>c1ccc(C(c2ccccn2)N2CCOCC2)cc1</smiles>
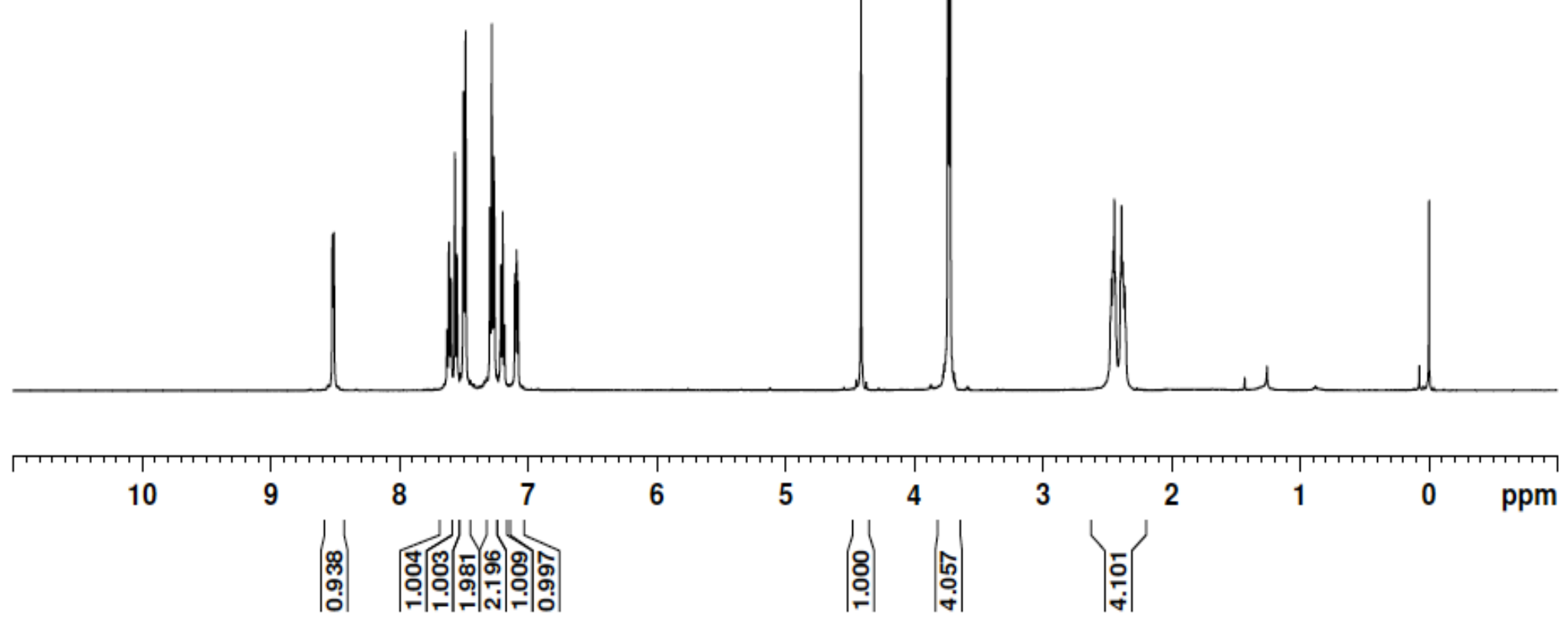

S 55 
${ }^{13} \mathrm{C}$ NMR (125 MHz) spectrum of $\mathbf{3 b}$.
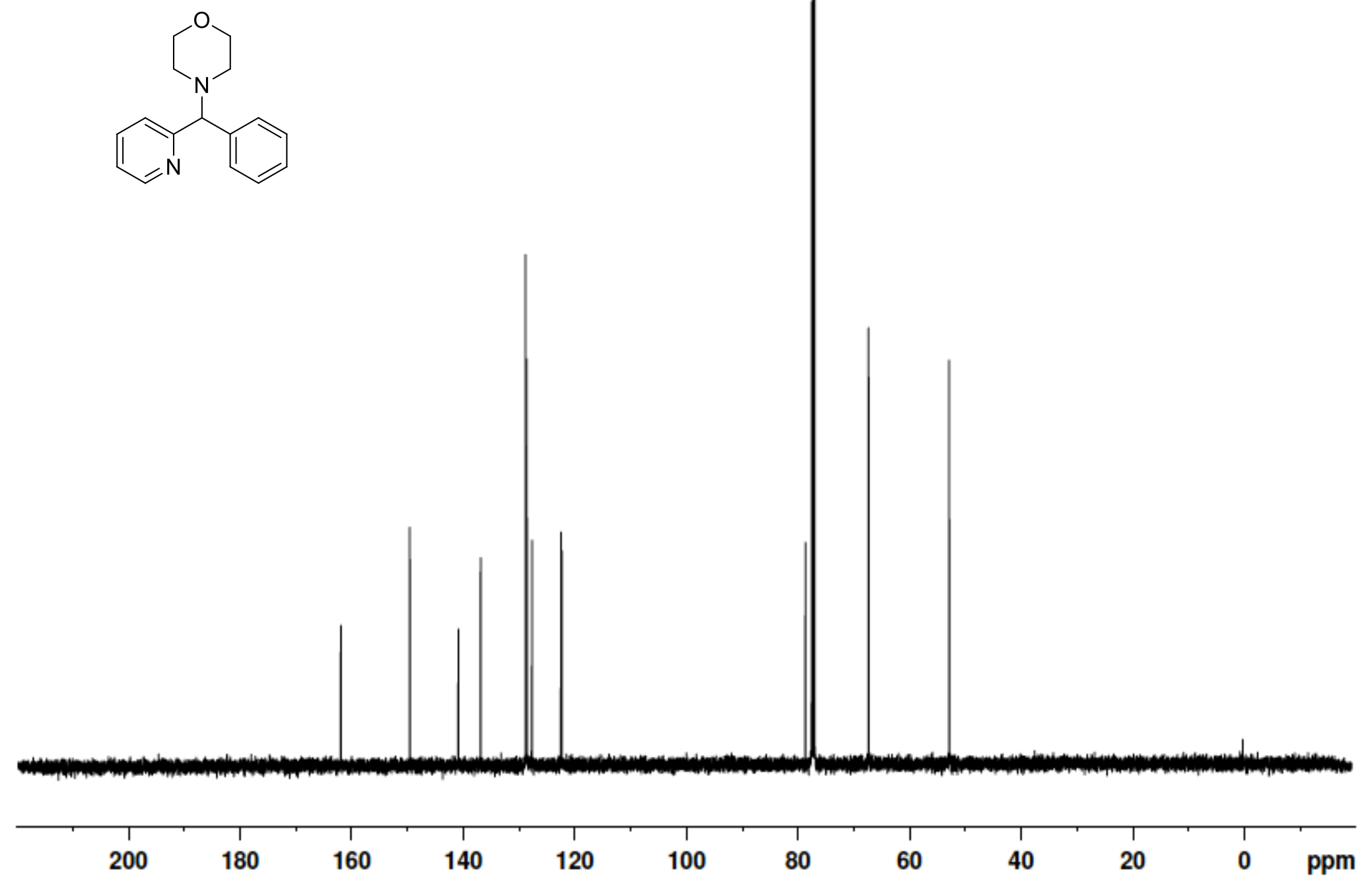
${ }^{1} \mathrm{H}$ NMR (500 MHz) spectrum of $3 \mathbf{c}$.

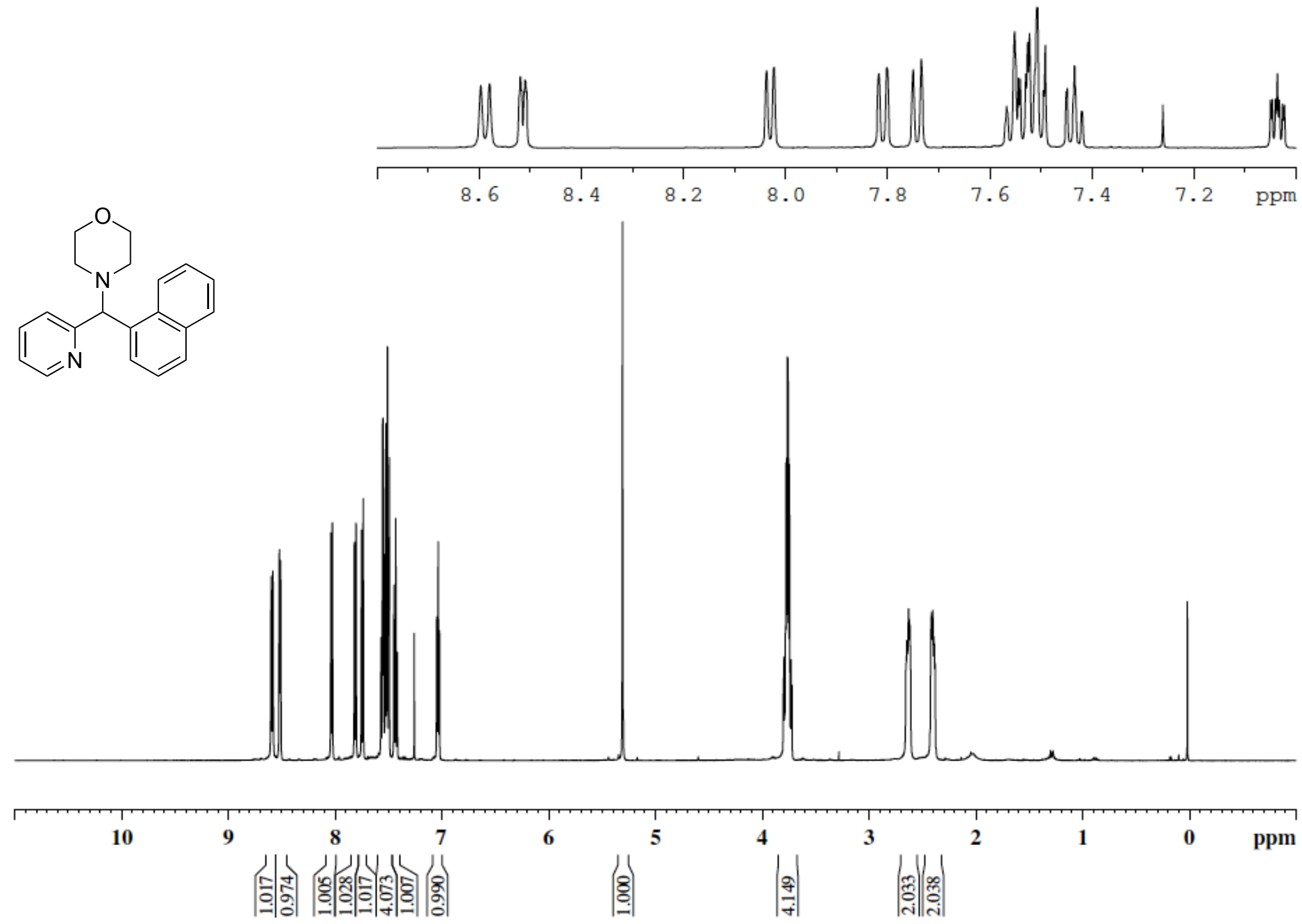


${ }^{13} \mathrm{C}$ NMR (125 MHz) spectrum of $\mathbf{3 c}$.
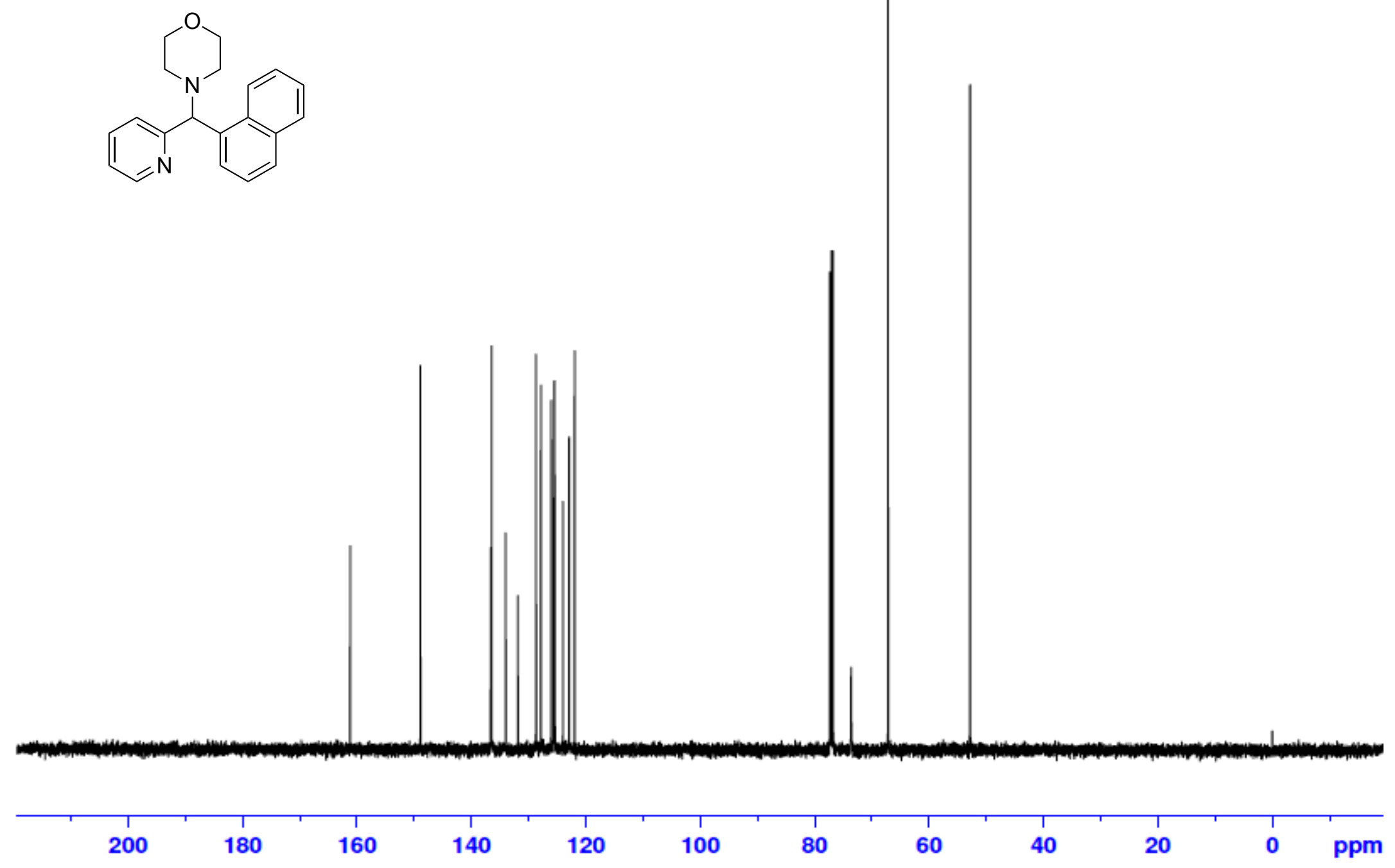
${ }^{1} \mathrm{H}$ NMR (500 MHz) spectrum of 3d.

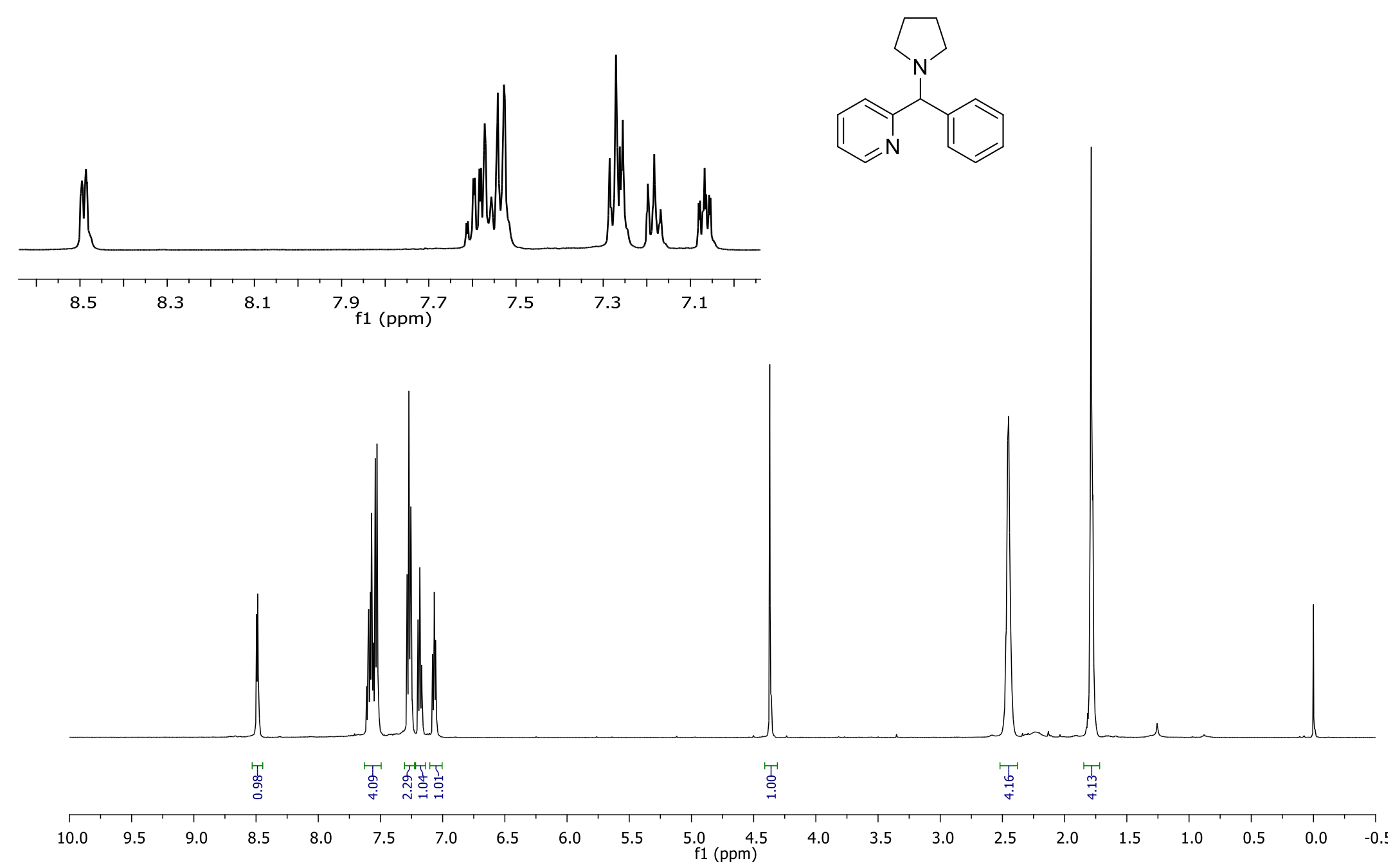


${ }^{13} \mathrm{C}$ NMR (125 MHz) spectrum of $\mathbf{3 d}$.
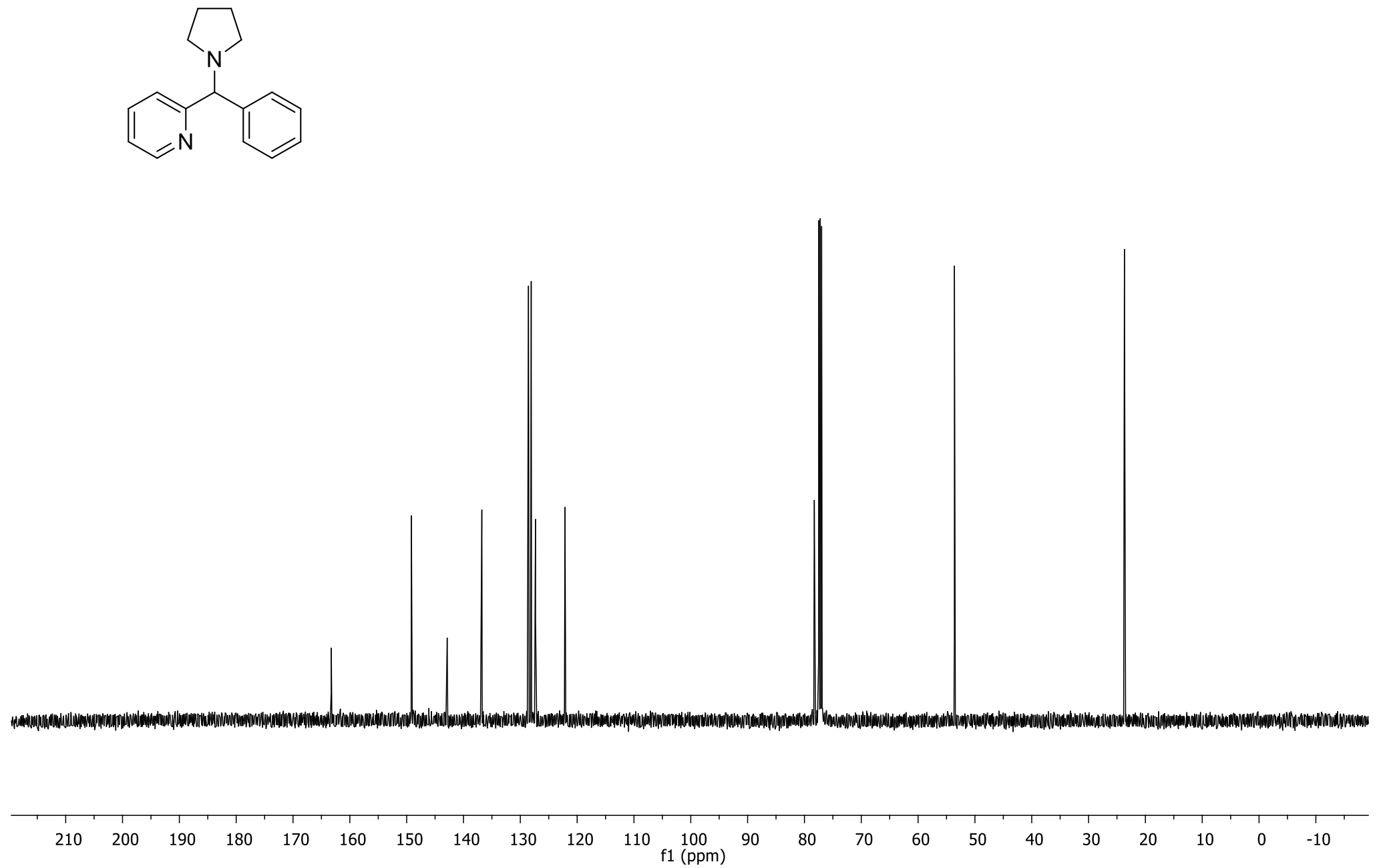

S 60 
${ }^{1} \mathrm{H}$ NMR (300 MHz) spectrum of $3 \mathbf{e}$.
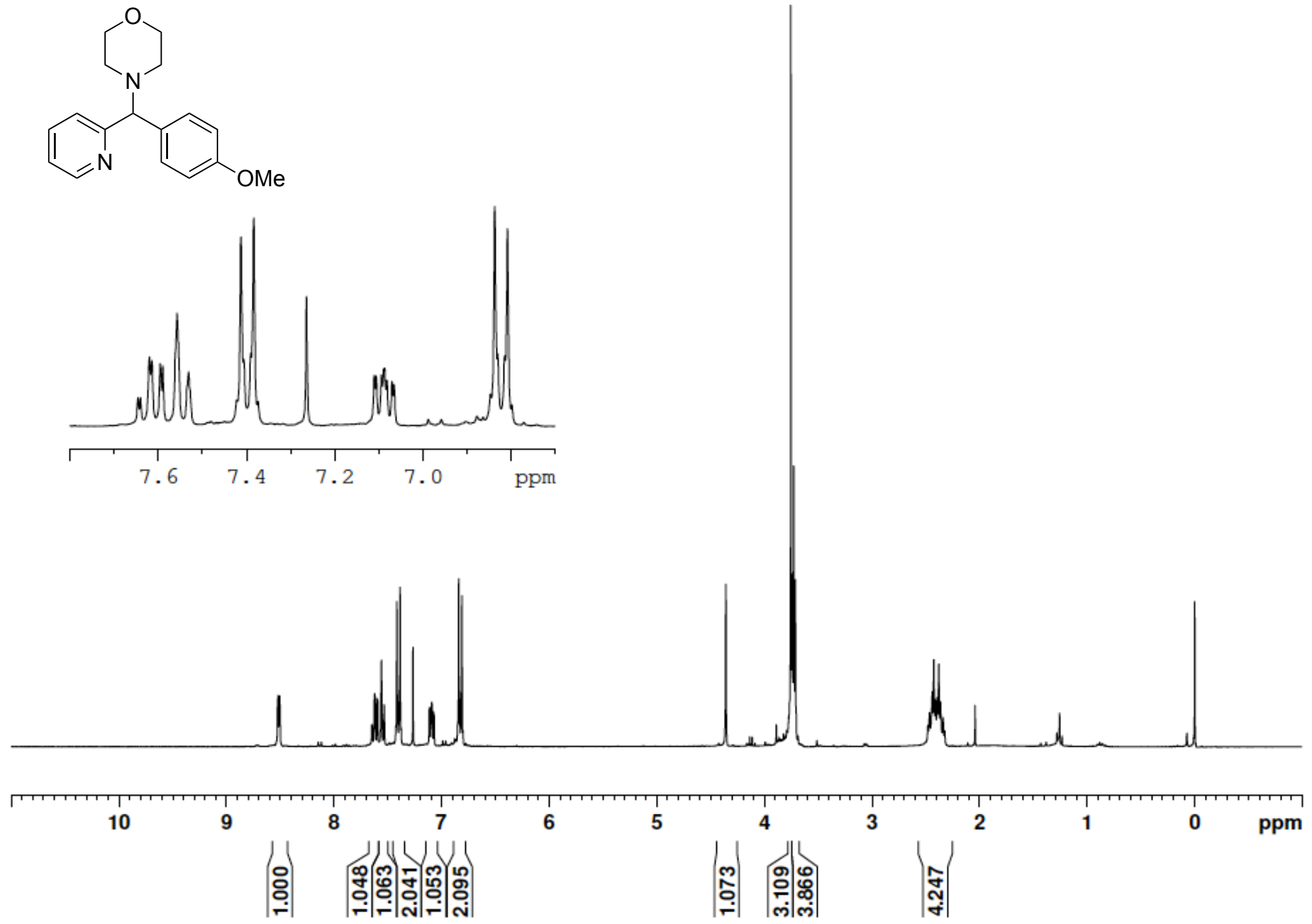
${ }^{13} \mathrm{C}$ NMR (75 MHz) spectrum of $\mathbf{3 e}$.
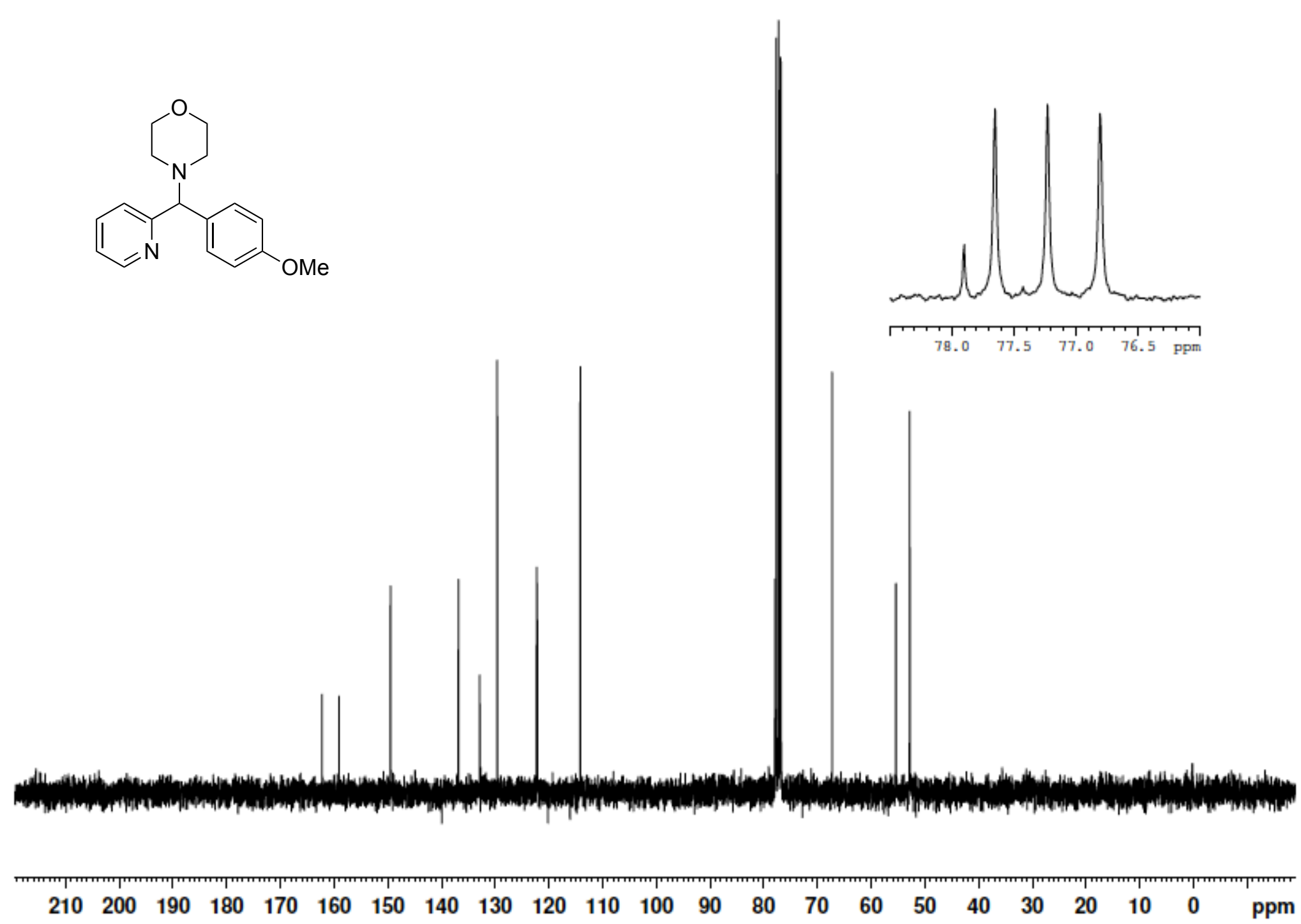
${ }^{1} \mathrm{H}$ NMR (500 MHz) spectrum of $\mathbf{3 f}$.

${ }^{13} \mathrm{C}$ NMR (125 MHz) spectrum of $\mathbf{3 f}$.
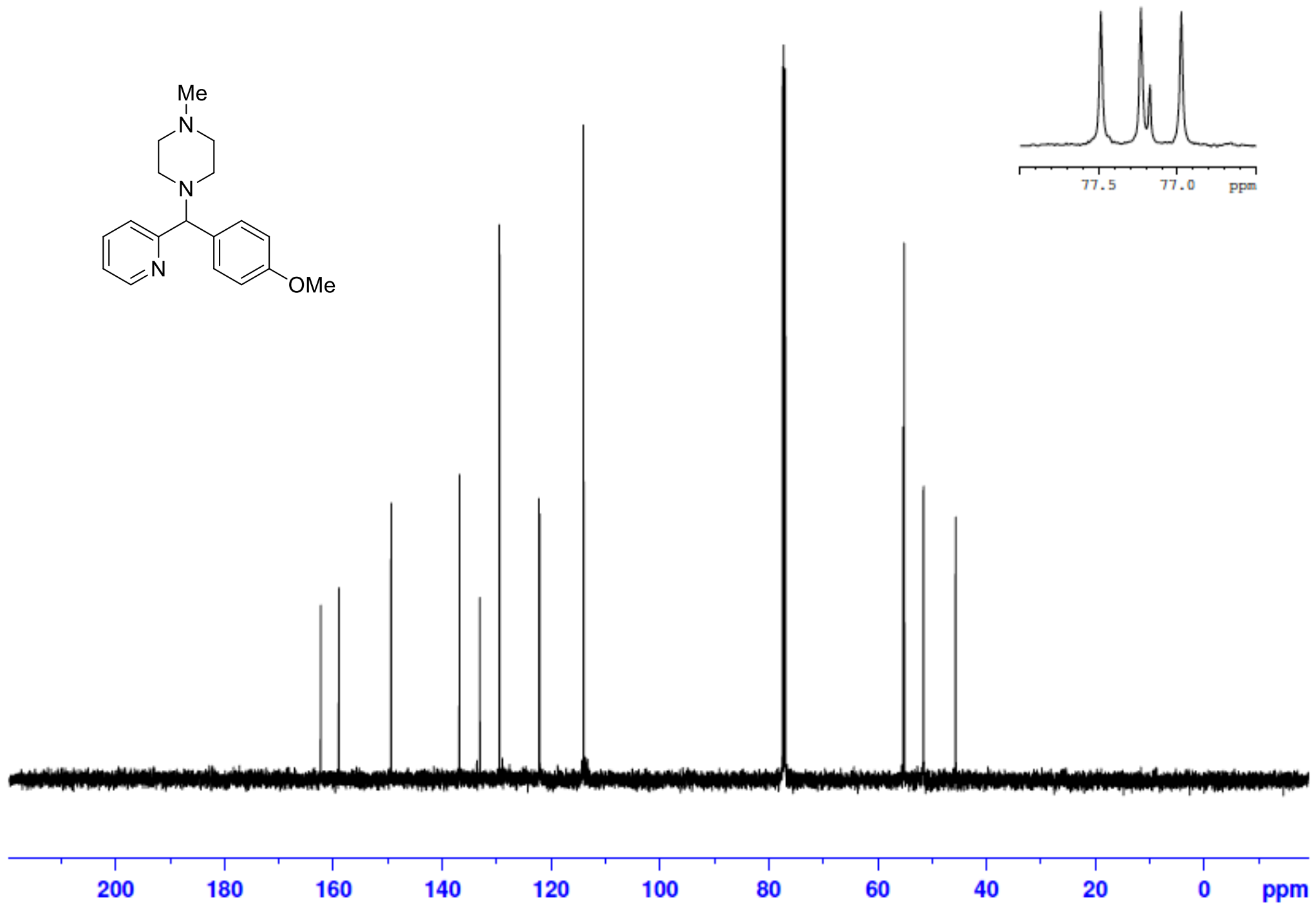

S 64 
${ }^{1} \mathrm{H}$ NMR (500 MHz) spectrum of $\mathbf{3 g}$.
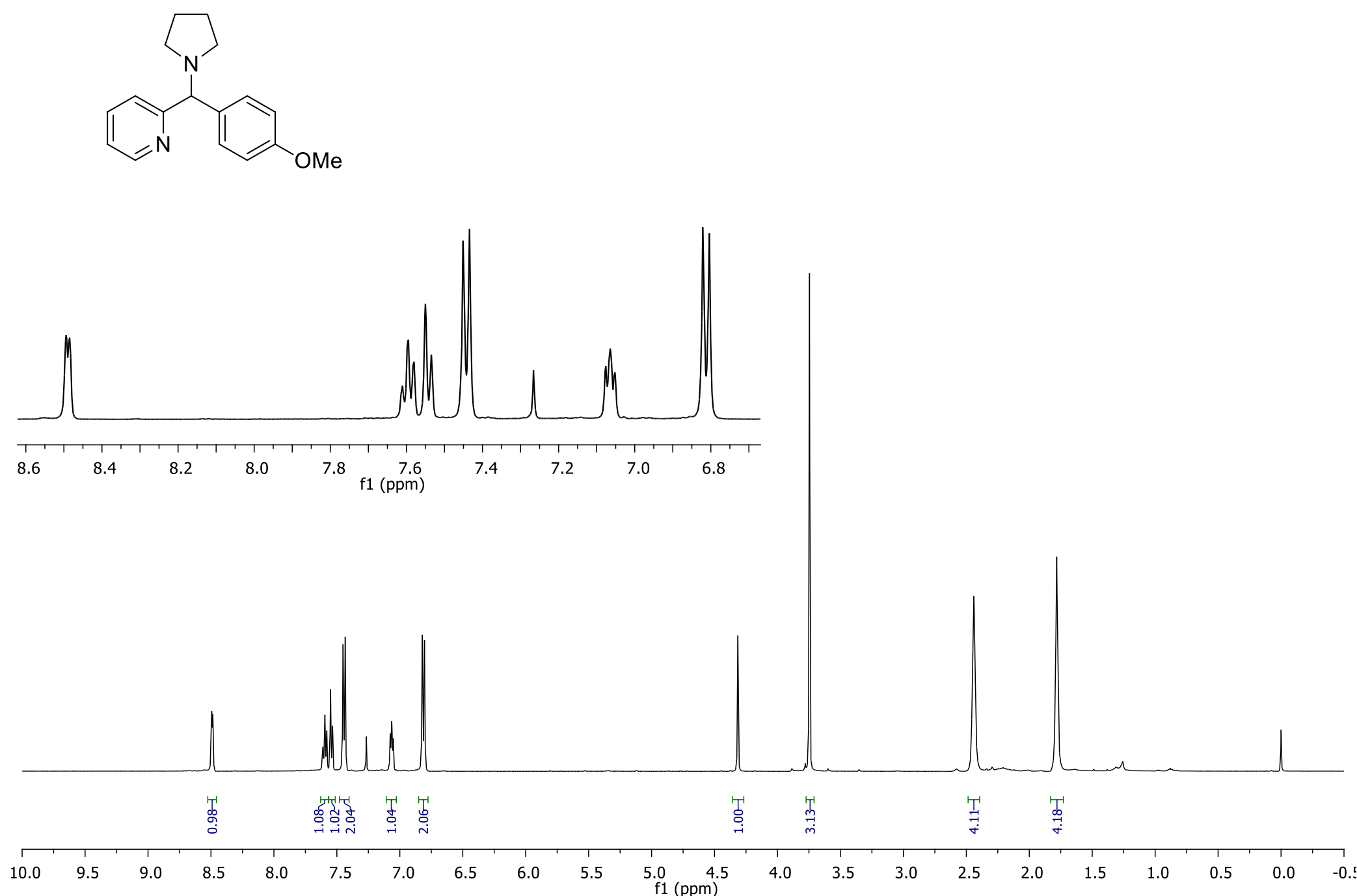
${ }^{13} \mathrm{C}$ NMR (125 MHz) spectrum of $\mathbf{3 g}$.

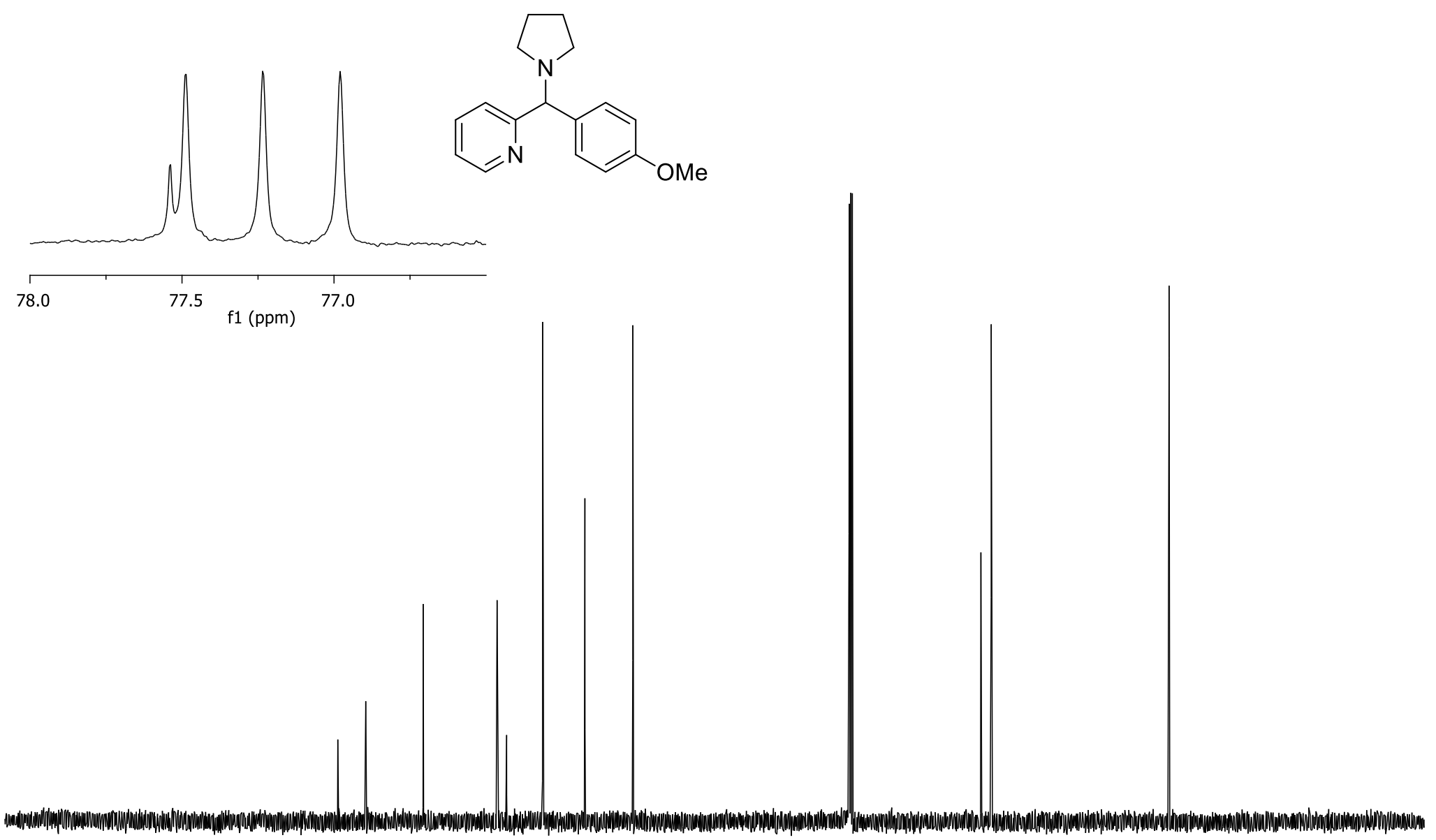

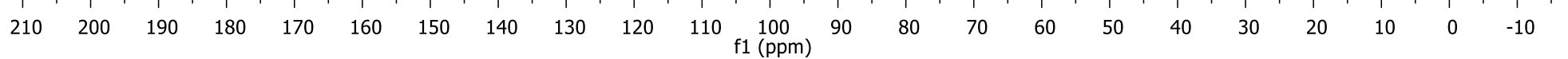


${ }^{1} \mathrm{H}$ NMR (500 MHz) spectrum of $\mathbf{3} \mathbf{h}$.<smiles>CNc1ccc(C(c2ccccn2)N2CCOCC2)cc1</smiles>
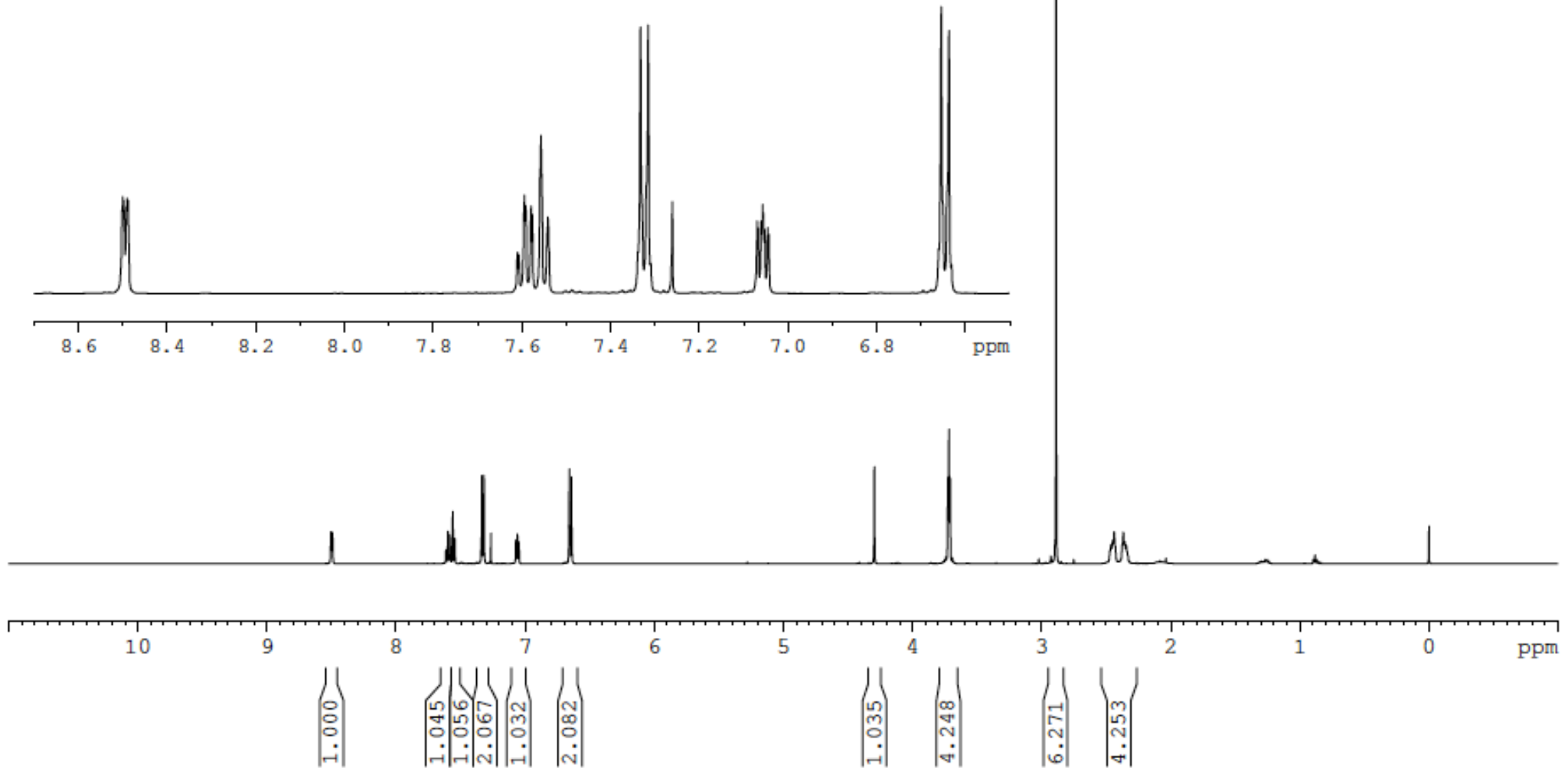

S 67 
${ }^{13} \mathrm{C}$ NMR (125 MHz) spectrum of $\mathbf{3 h}$.

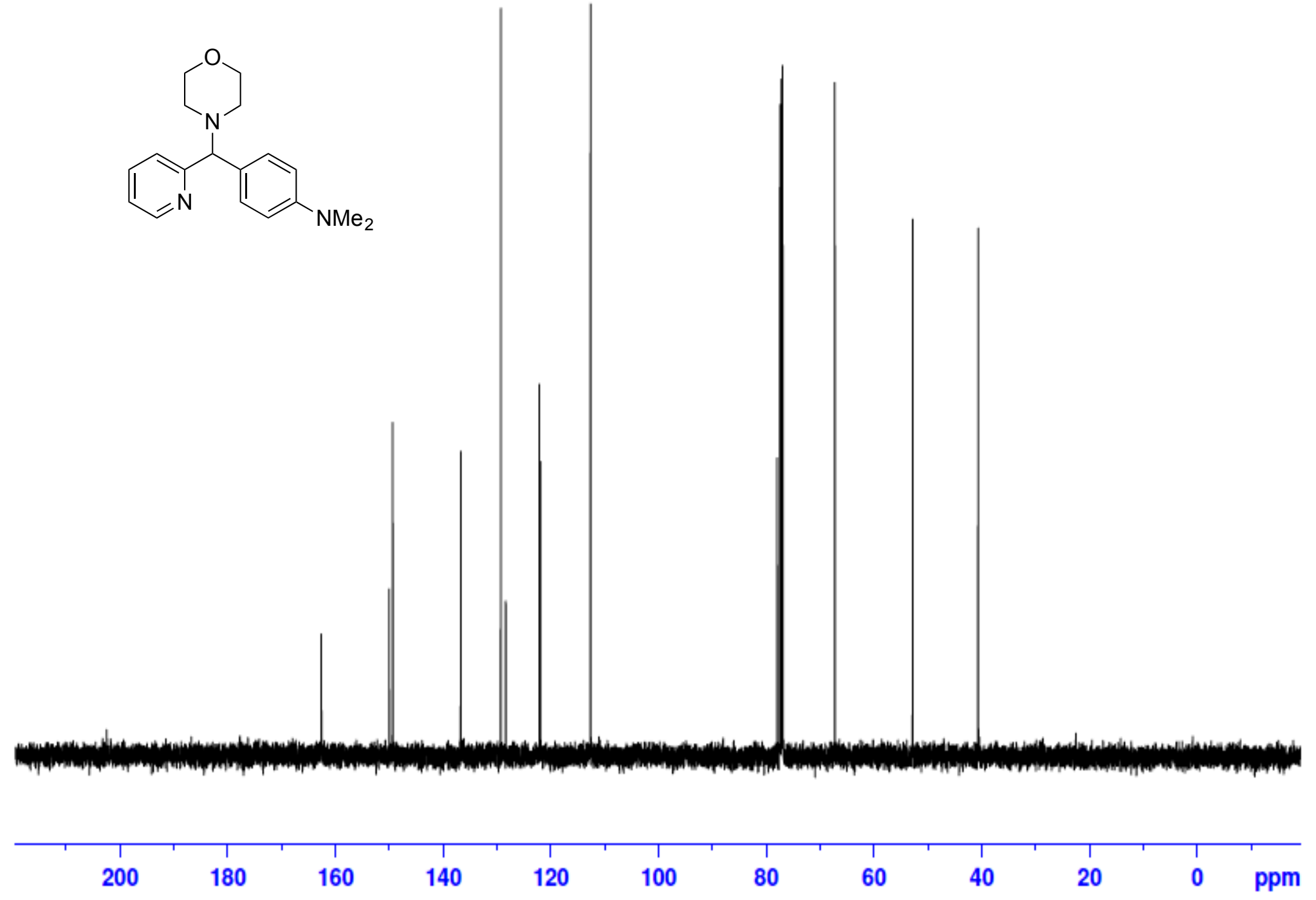

S 68 
${ }^{1} \mathrm{H}$ NMR (500 MHz) spectrum of $3 \mathbf{i}$.

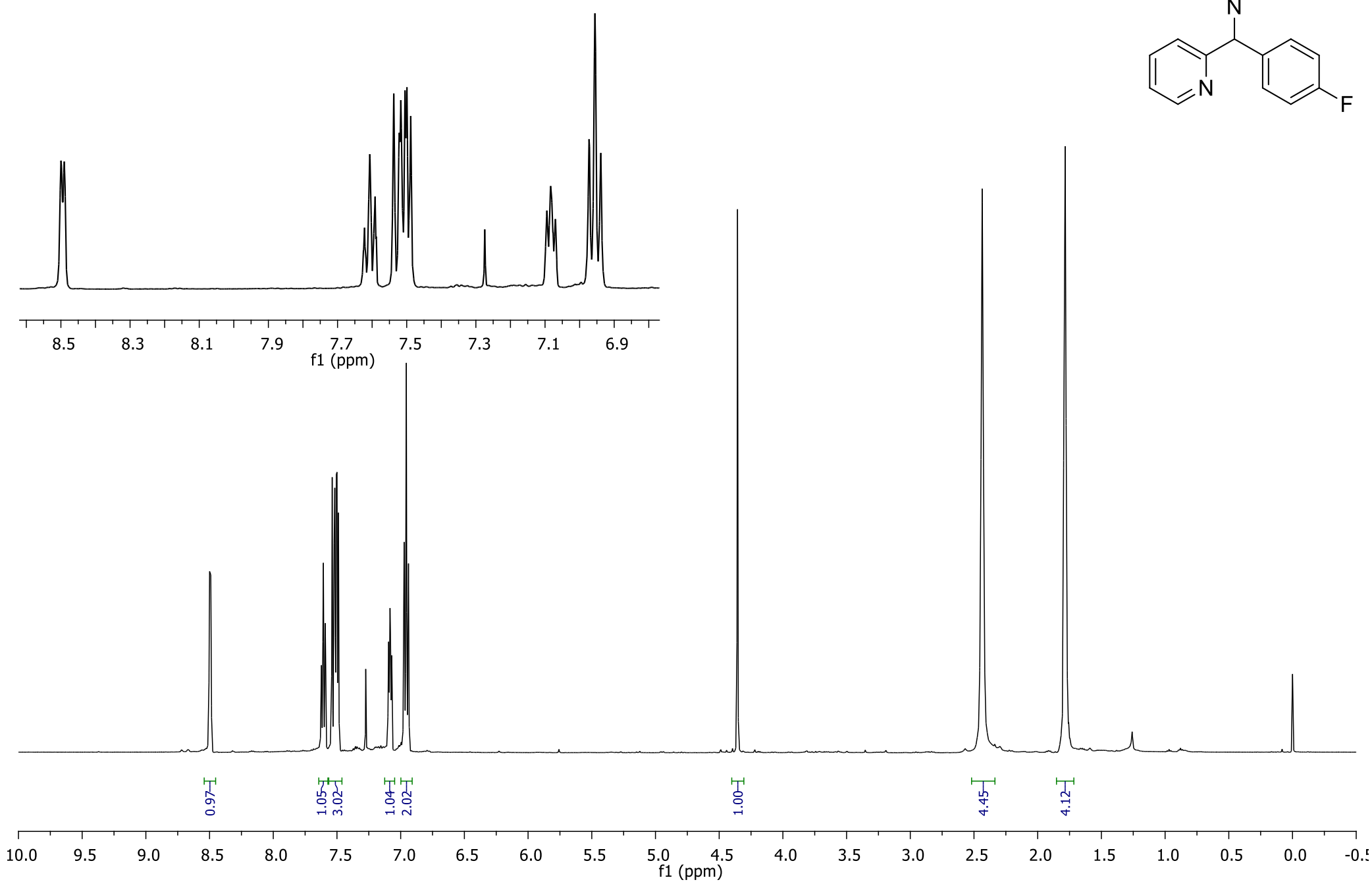

S 69 
${ }^{13} \mathrm{C}$ NMR (125 MHz) spectrum of $\mathbf{3 i}$.
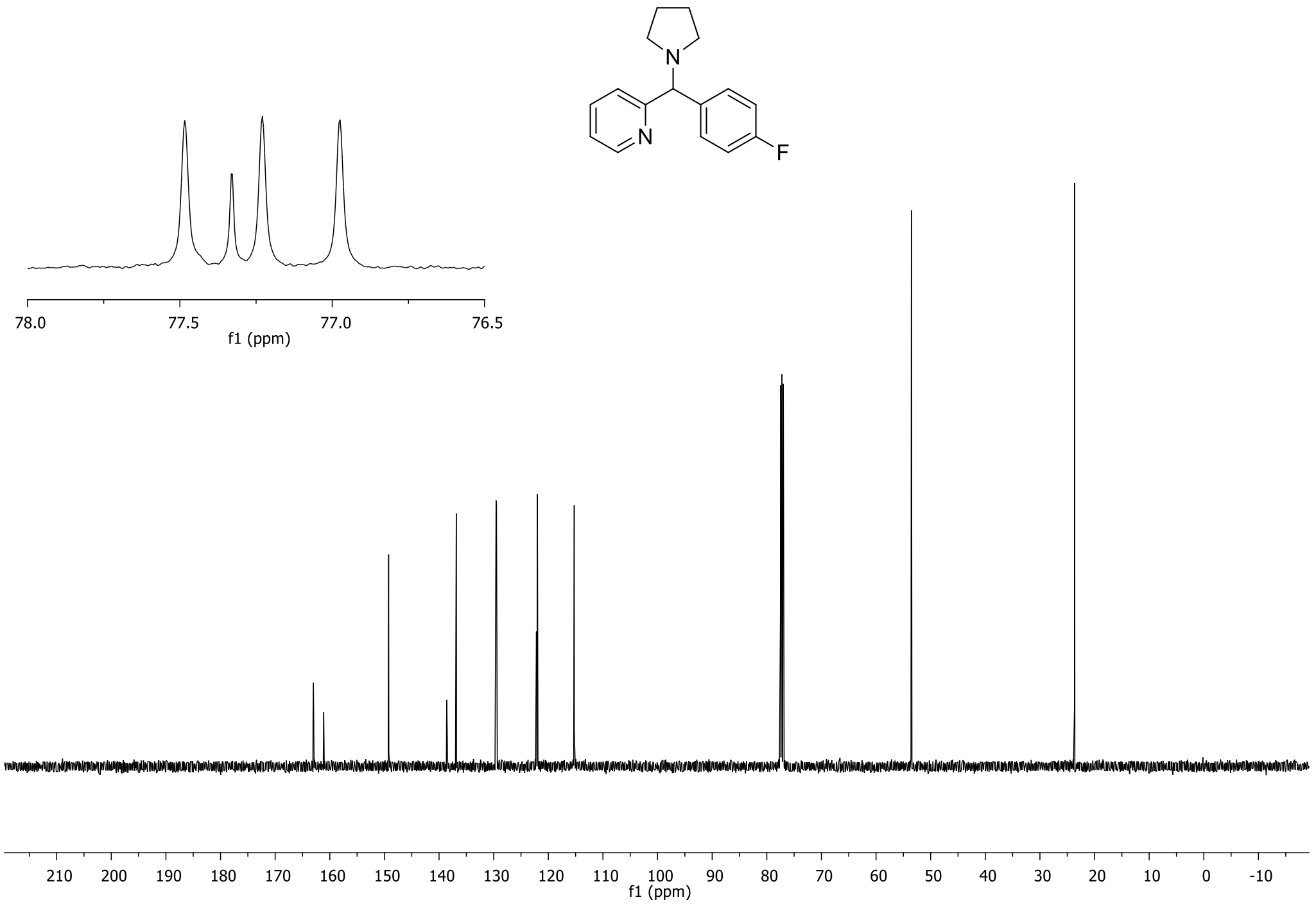

S 70 
${ }^{1} \mathrm{H}$ NMR (500 MHz) spectrum of $\mathbf{3 j}$.
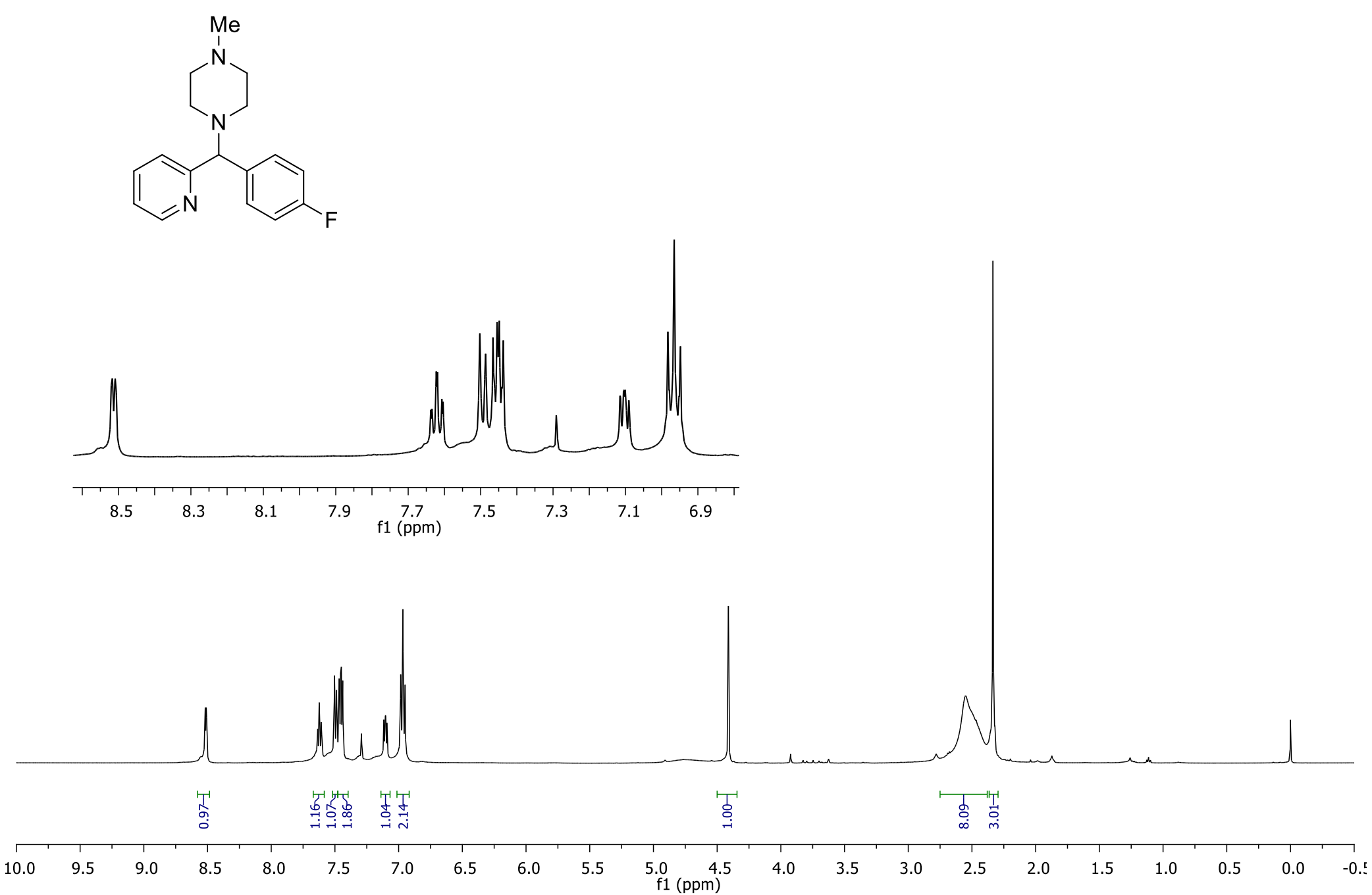
${ }^{13} \mathrm{C}$ NMR (125 MHz) spectrum of $\mathbf{3 j}$.
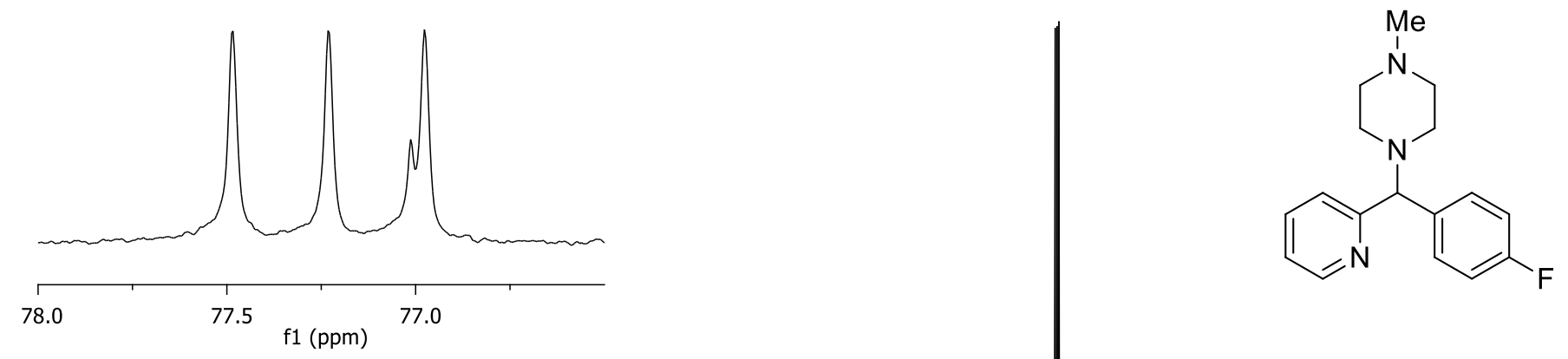

W(IN)

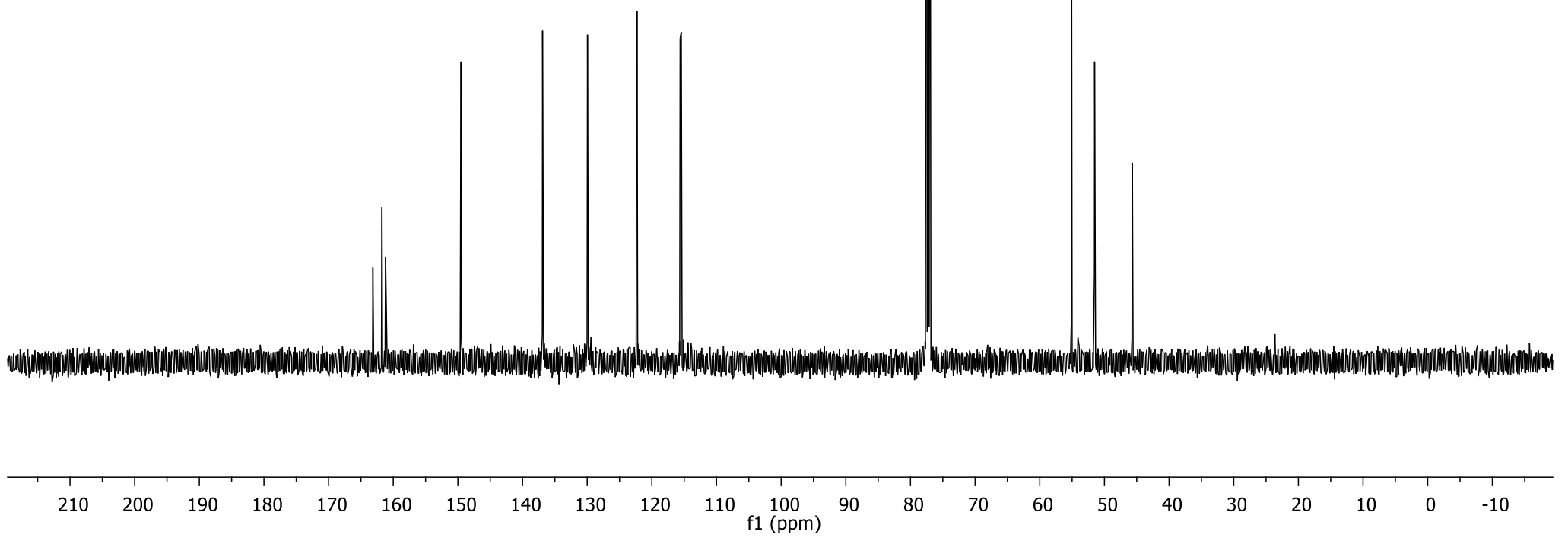


${ }^{1} \mathrm{H}$ NMR (500 MHz) spectrum of $\mathbf{3 k}$.

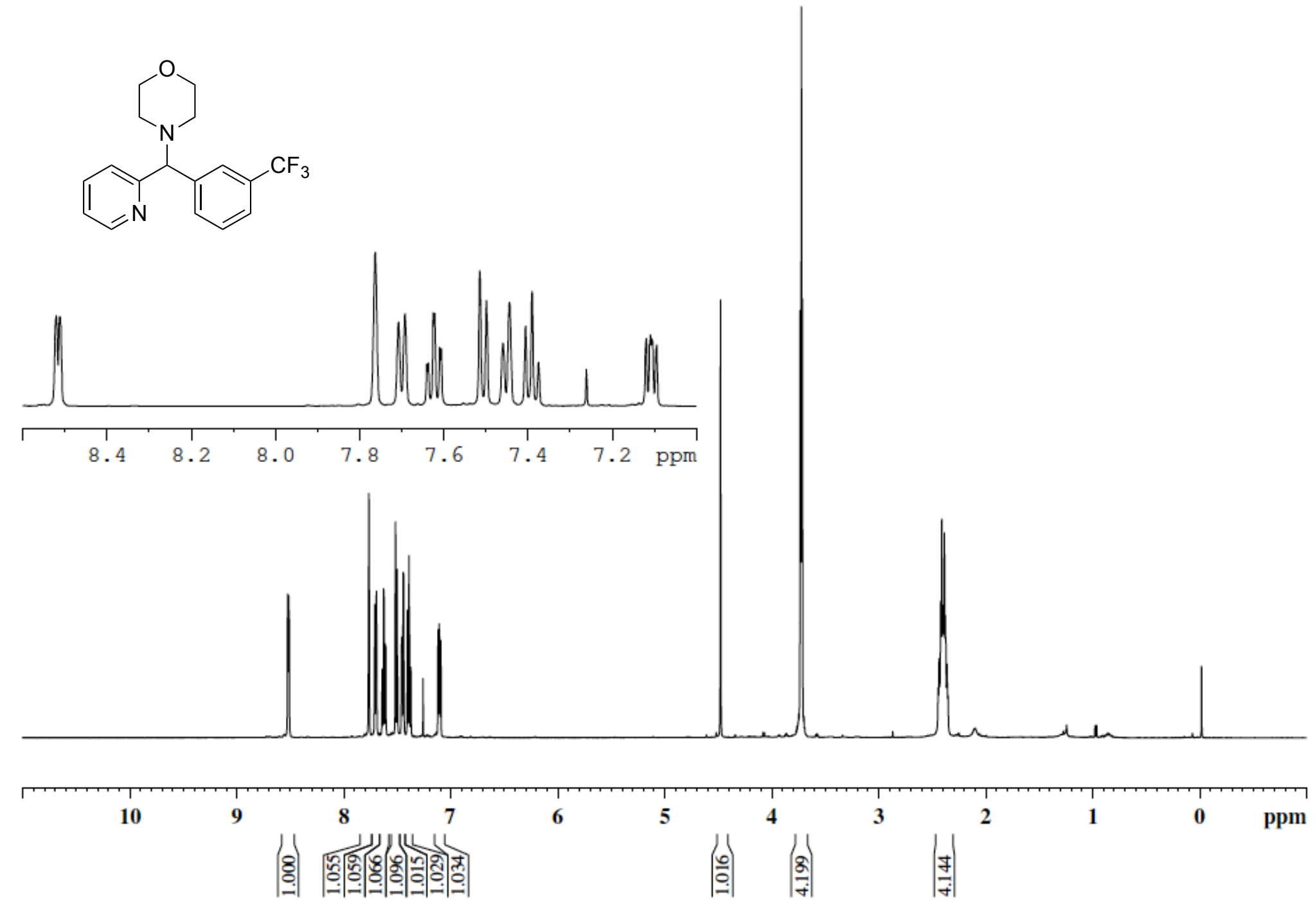


${ }^{13} \mathrm{C}$ NMR (125 MHz) spectrum of $\mathbf{3 k}$.
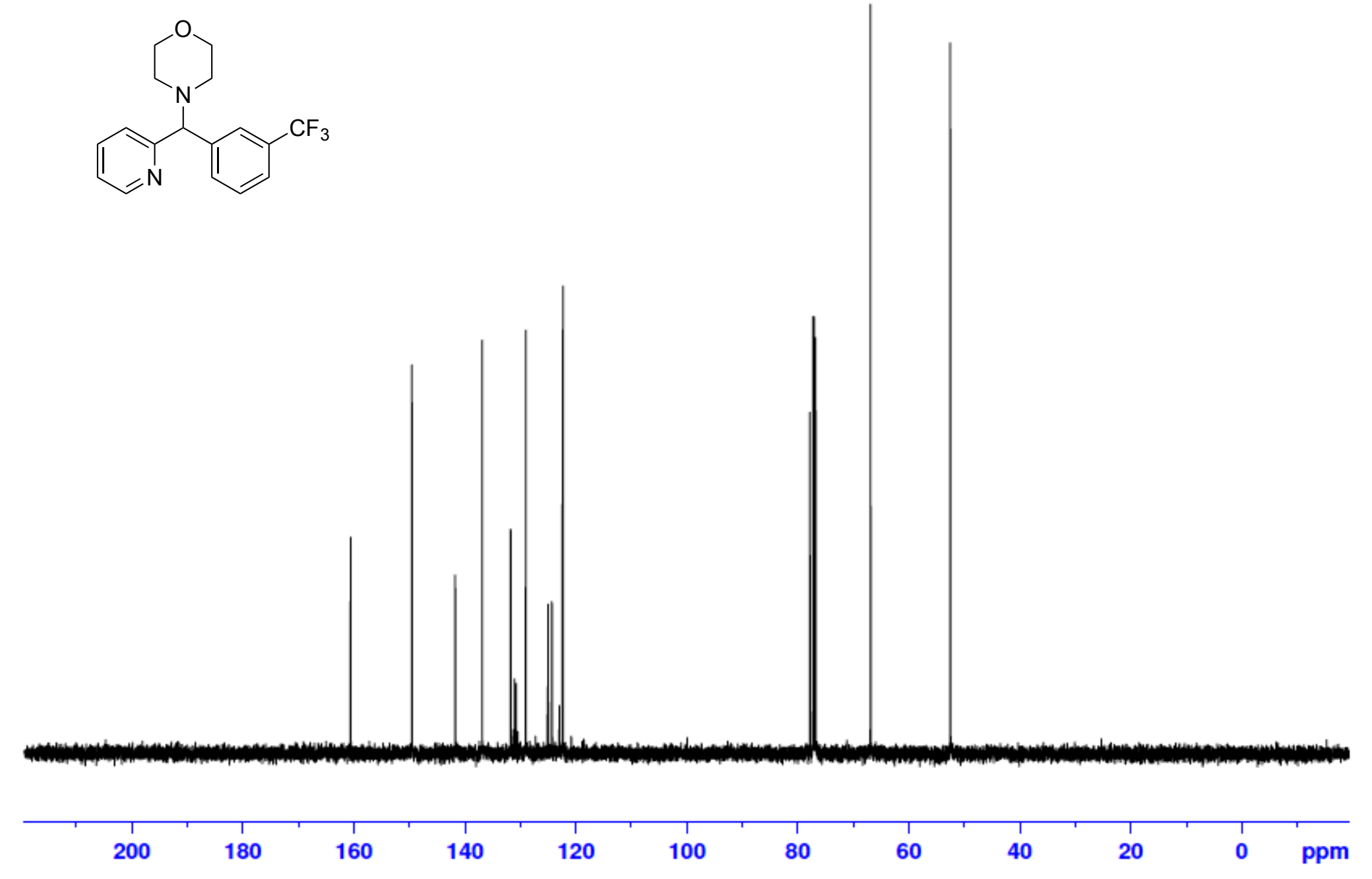
${ }^{1} \mathrm{H}$ NMR (500 MHz) spectrum of $\mathbf{3 1}$.
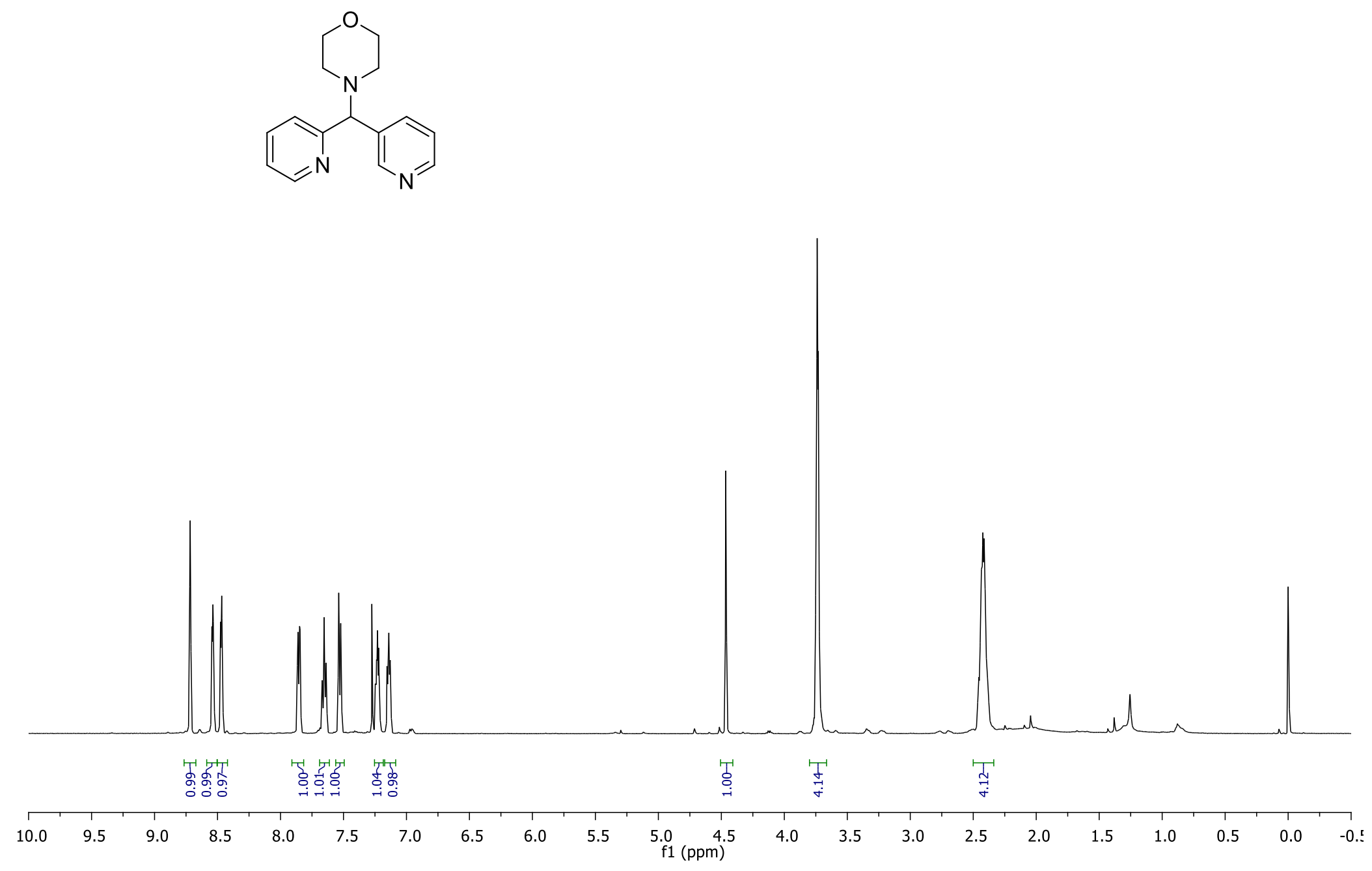
${ }^{13} \mathrm{C}$ NMR (125 MHz) spectrum of $3 \mathbf{3}$.

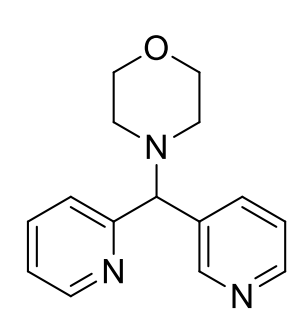

.

$170 \quad 160 \quad 150$

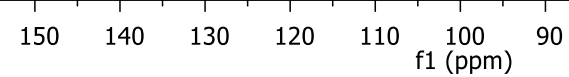

80 
${ }^{1} \mathrm{H}$ NMR (500 MHz) spectrum of $\mathbf{3 m}$.

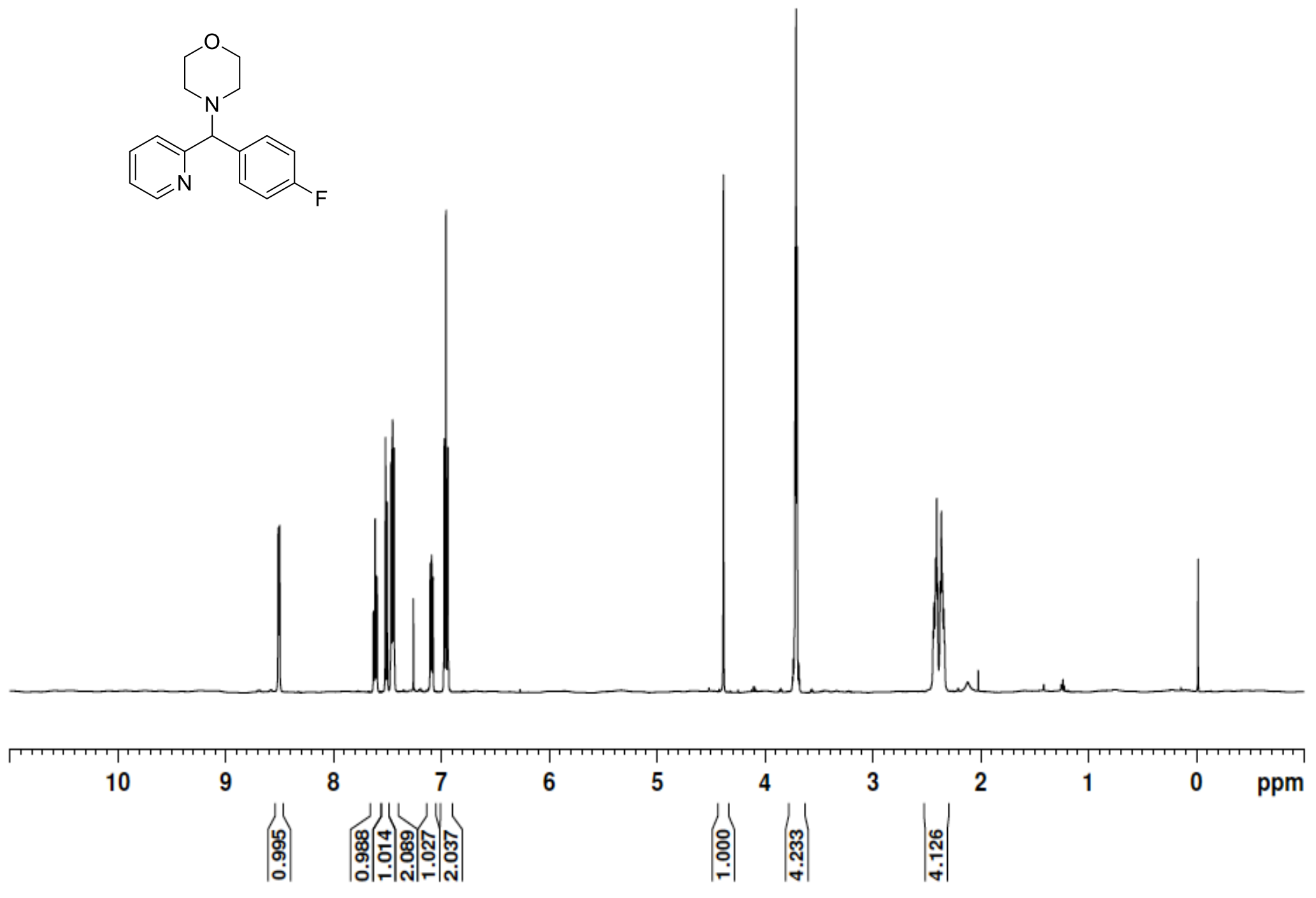


${ }^{13} \mathrm{C}$ NMR (125 MHz) spectrum of $\mathbf{3 m}$.
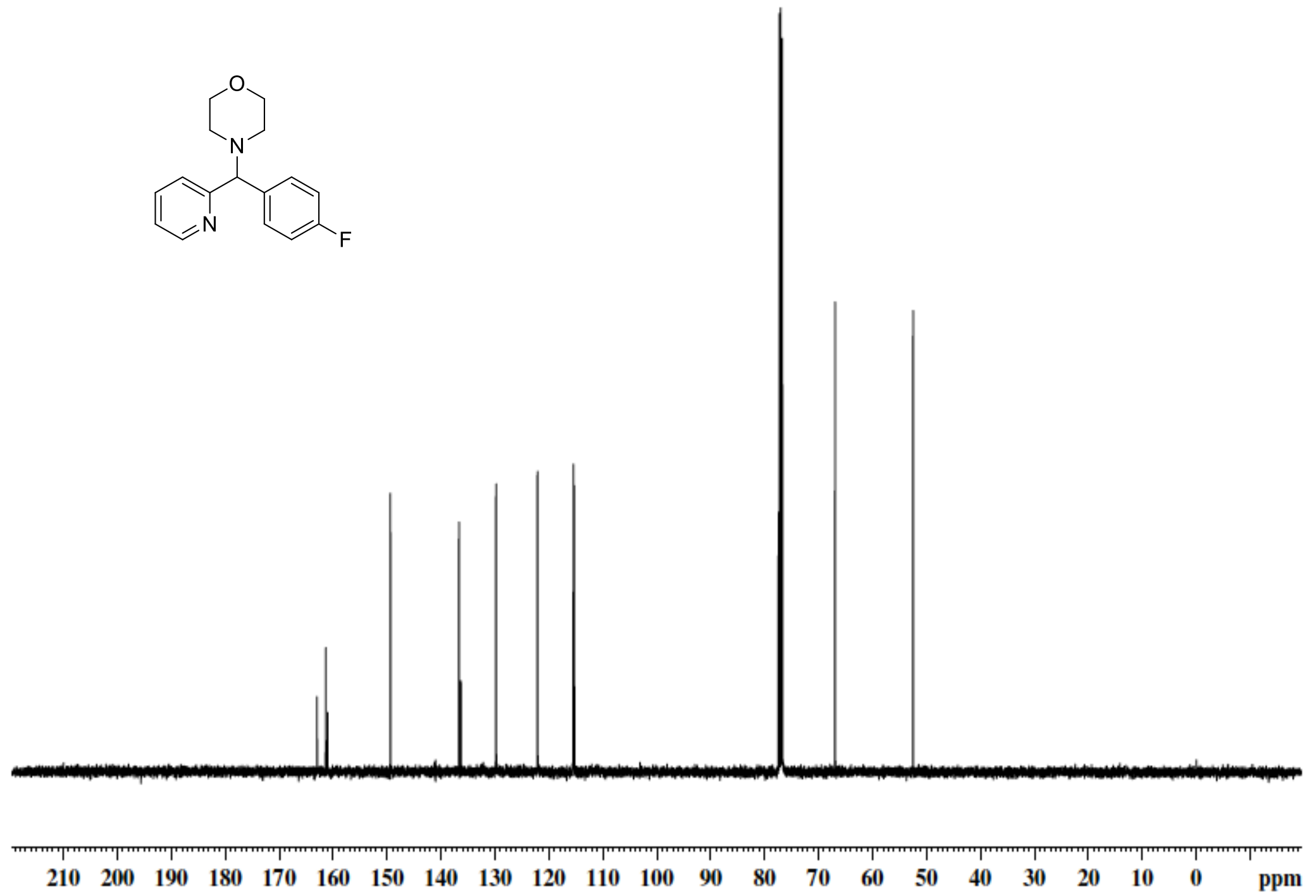
${ }^{1} \mathrm{H}$ NMR (500 MHz) spectrum of $\mathbf{4 a}$.
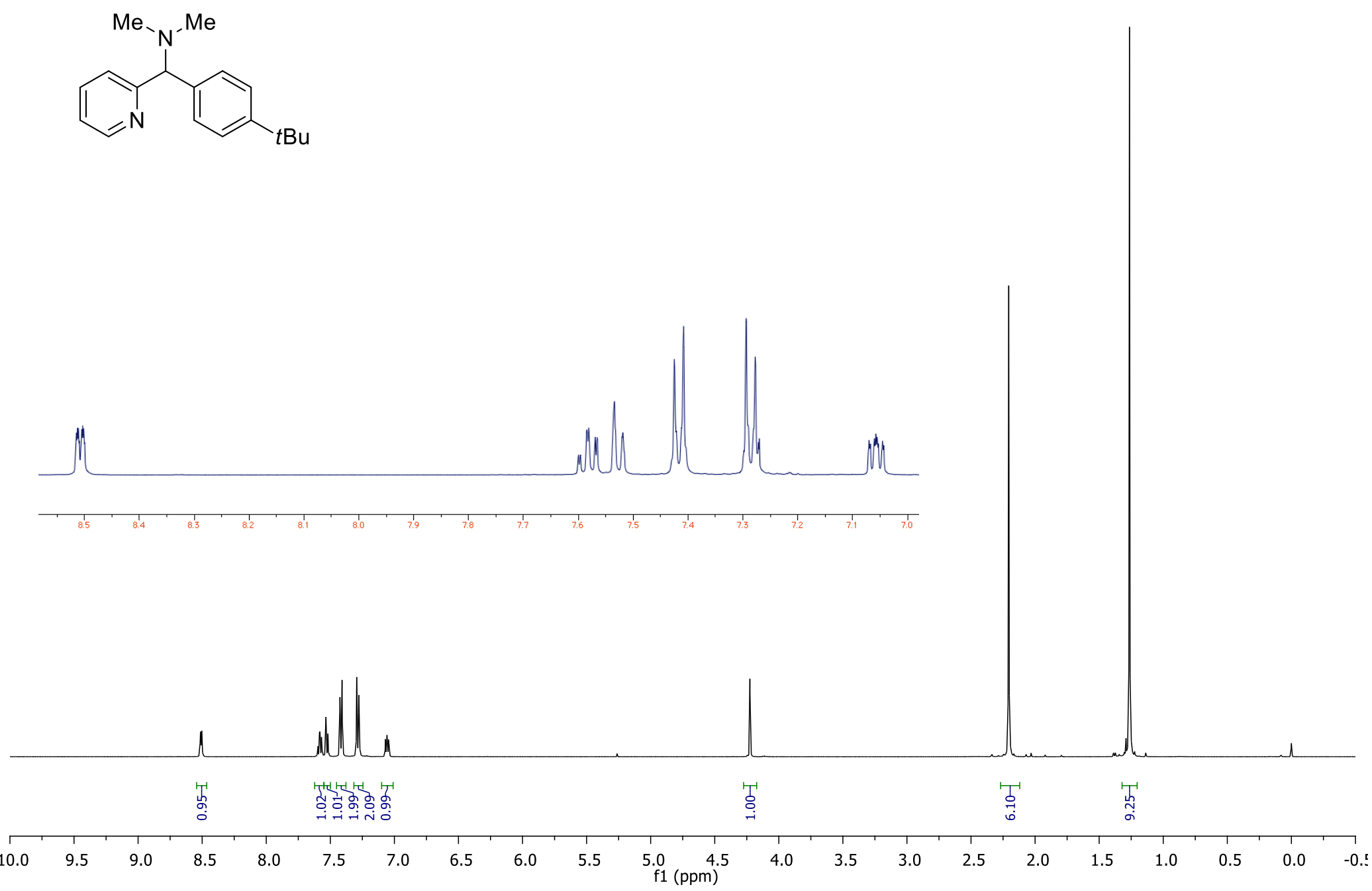
${ }^{13} \mathrm{C}$ NMR (125 MHz) spectrum of $\mathbf{4 a}$.
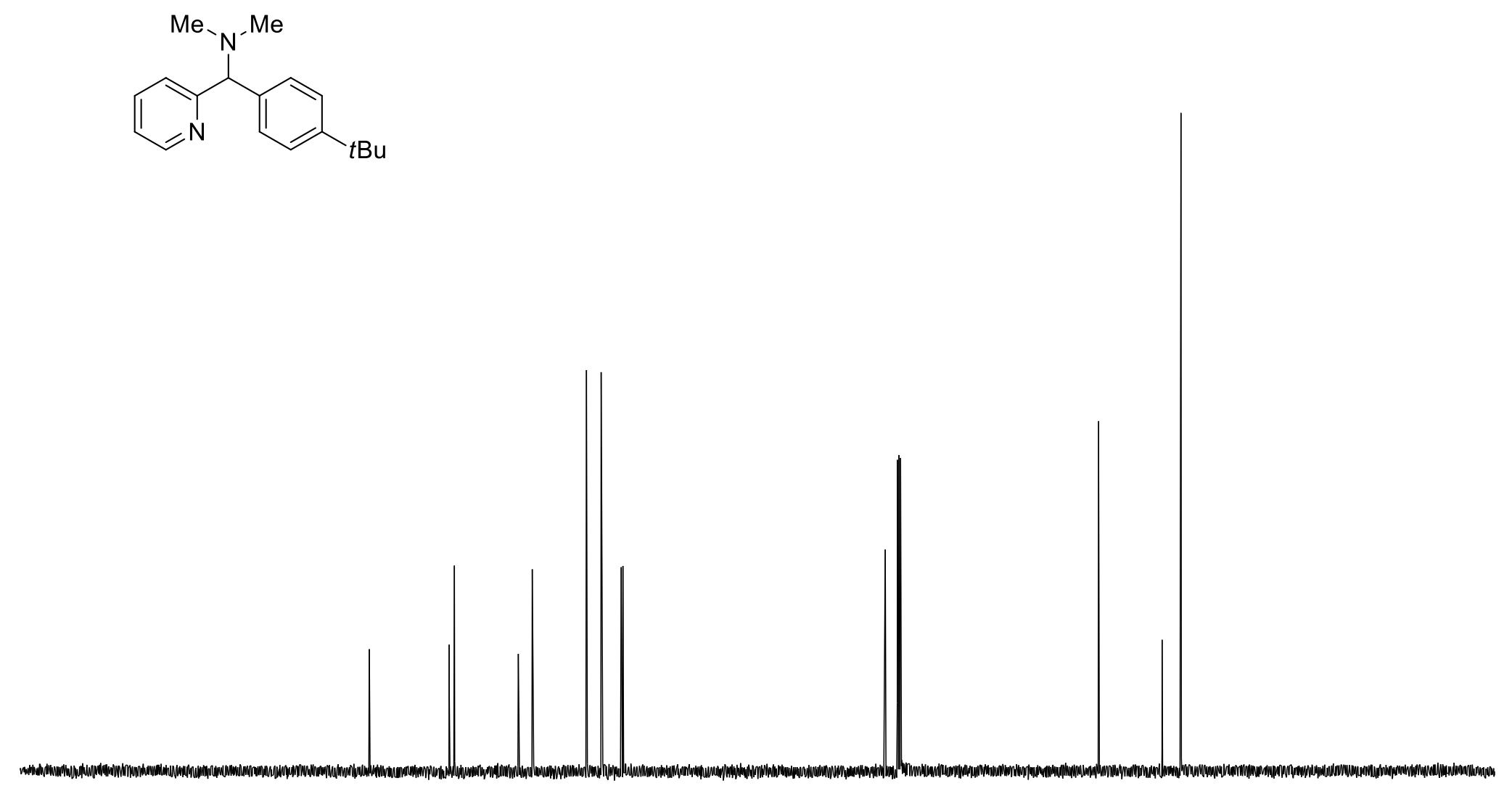

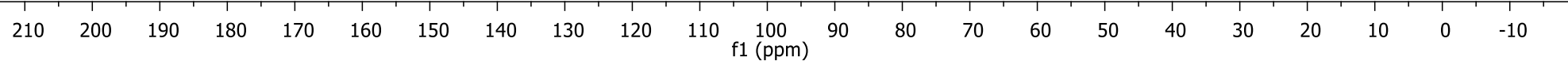


${ }^{1} \mathrm{H}$ NMR (500 MHz) spectrum of $\mathbf{4 b}$.
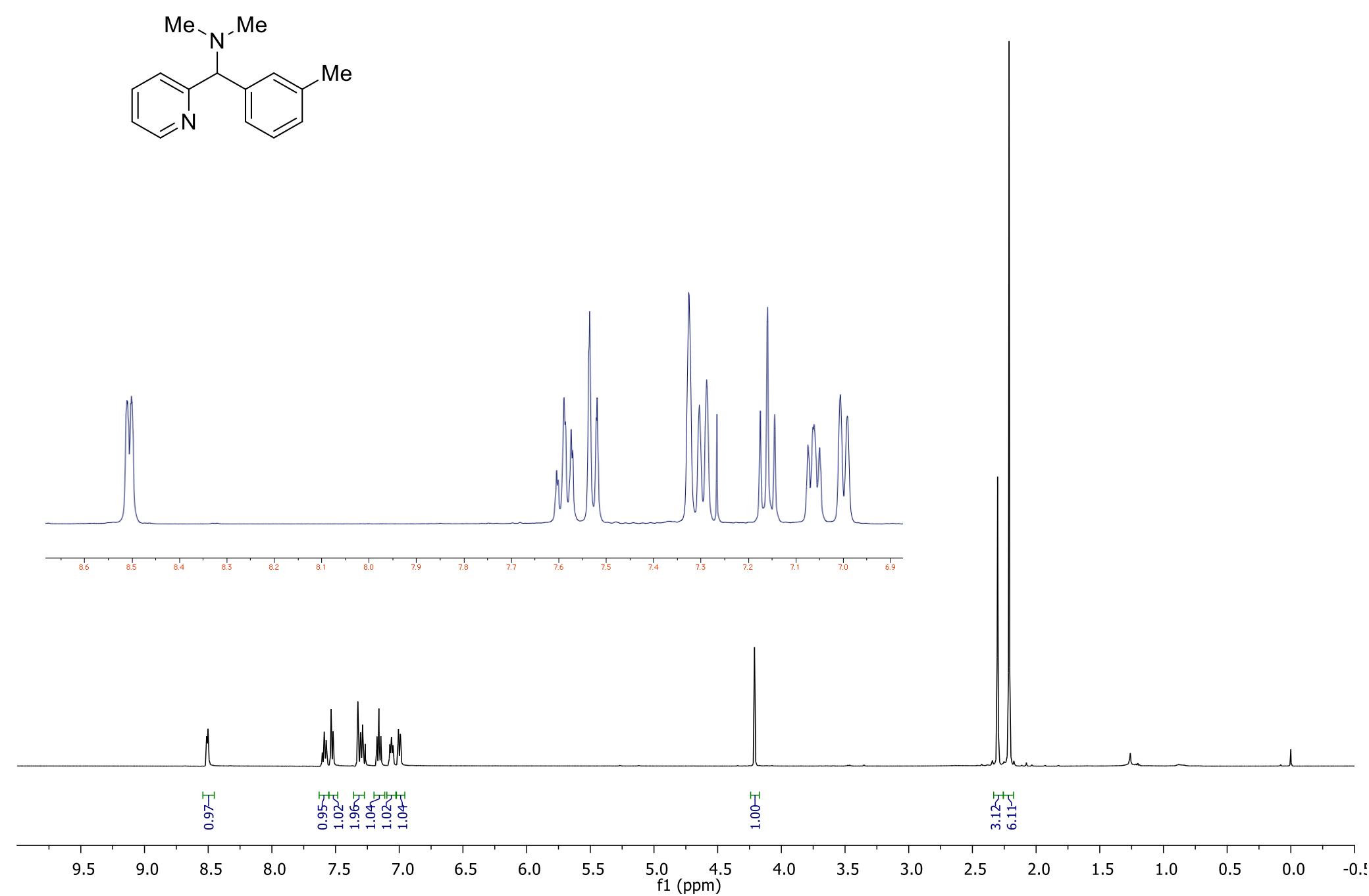
${ }^{13} \mathrm{C}$ NMR (125 MHz) spectrum of $\mathbf{4 b}$.

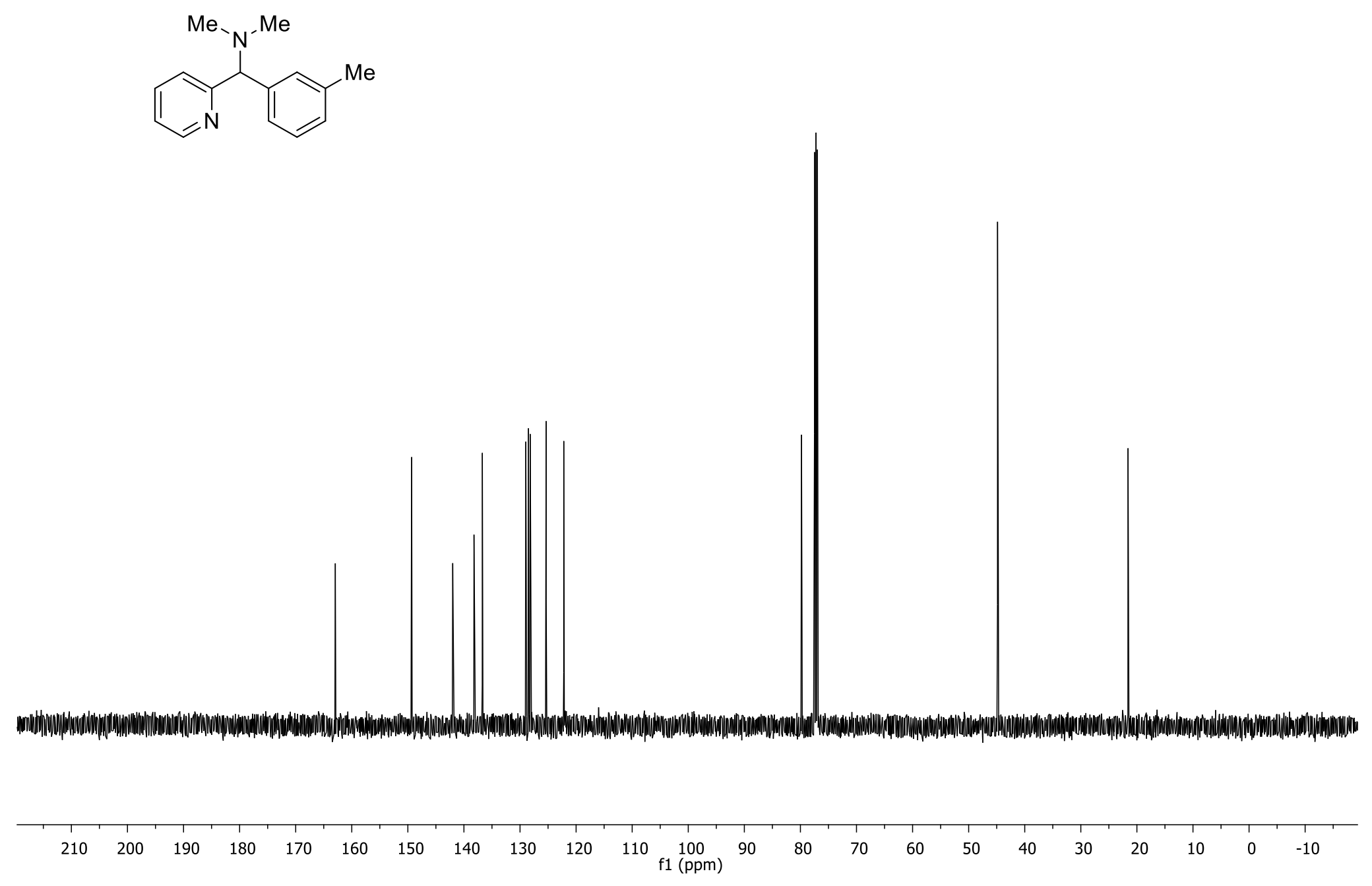


${ }^{1}$ H NMR (500 MHz) spectrum of 4c.
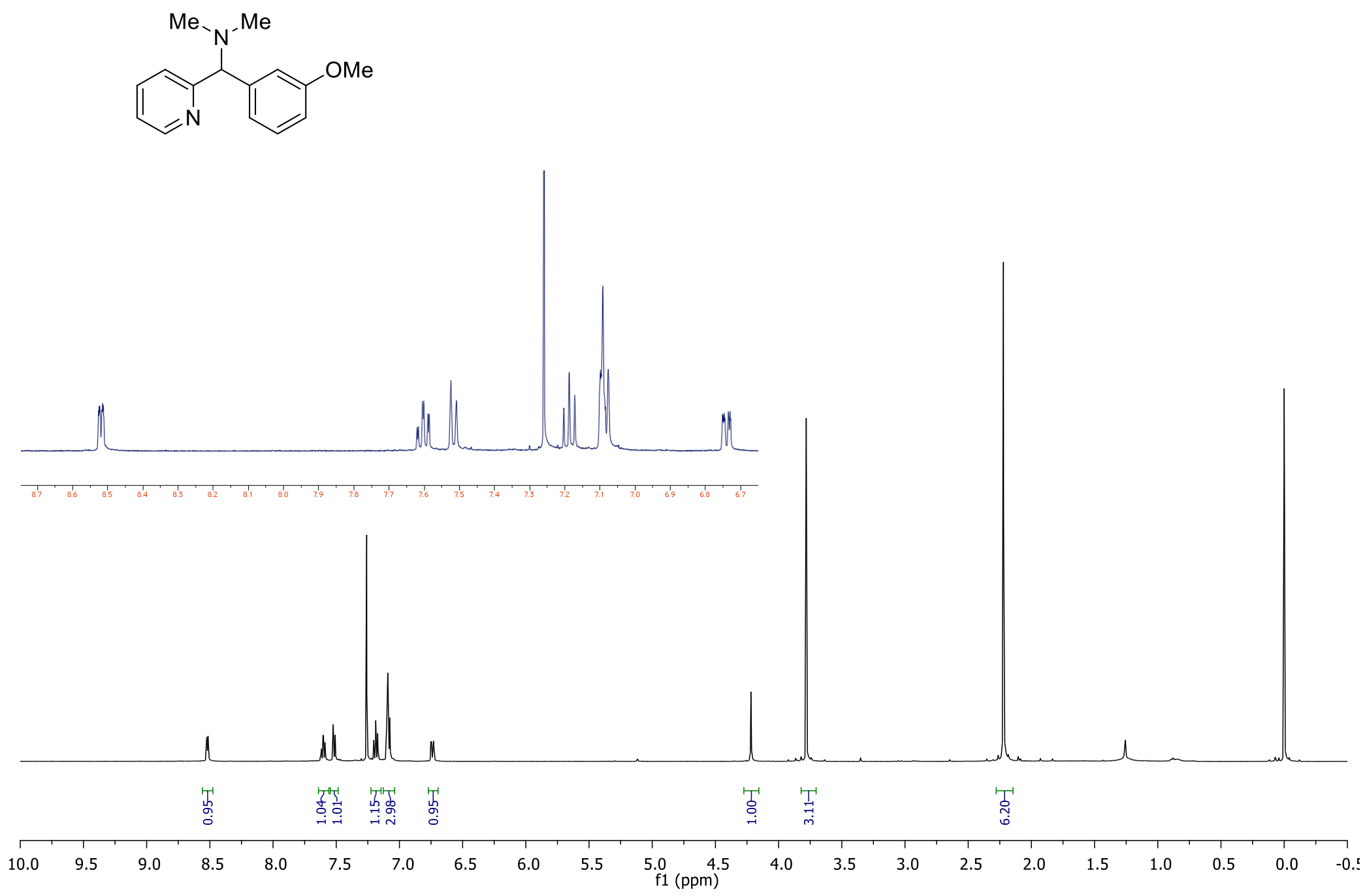
${ }^{13} \mathrm{C}$ NMR (125 MHz) spectrum of $4 \mathbf{c}$.<smiles>COc1cccc(C(c2ccccn2)N(C)C)c1</smiles>

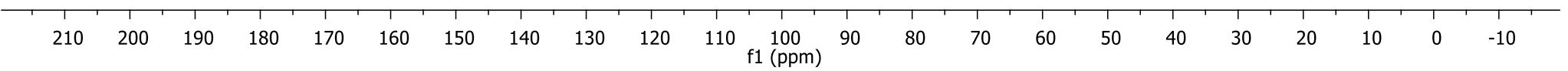

S 84 
${ }^{1} \mathrm{H}$ NMR (500 MHz) spectrum of $\mathbf{4 d}$.
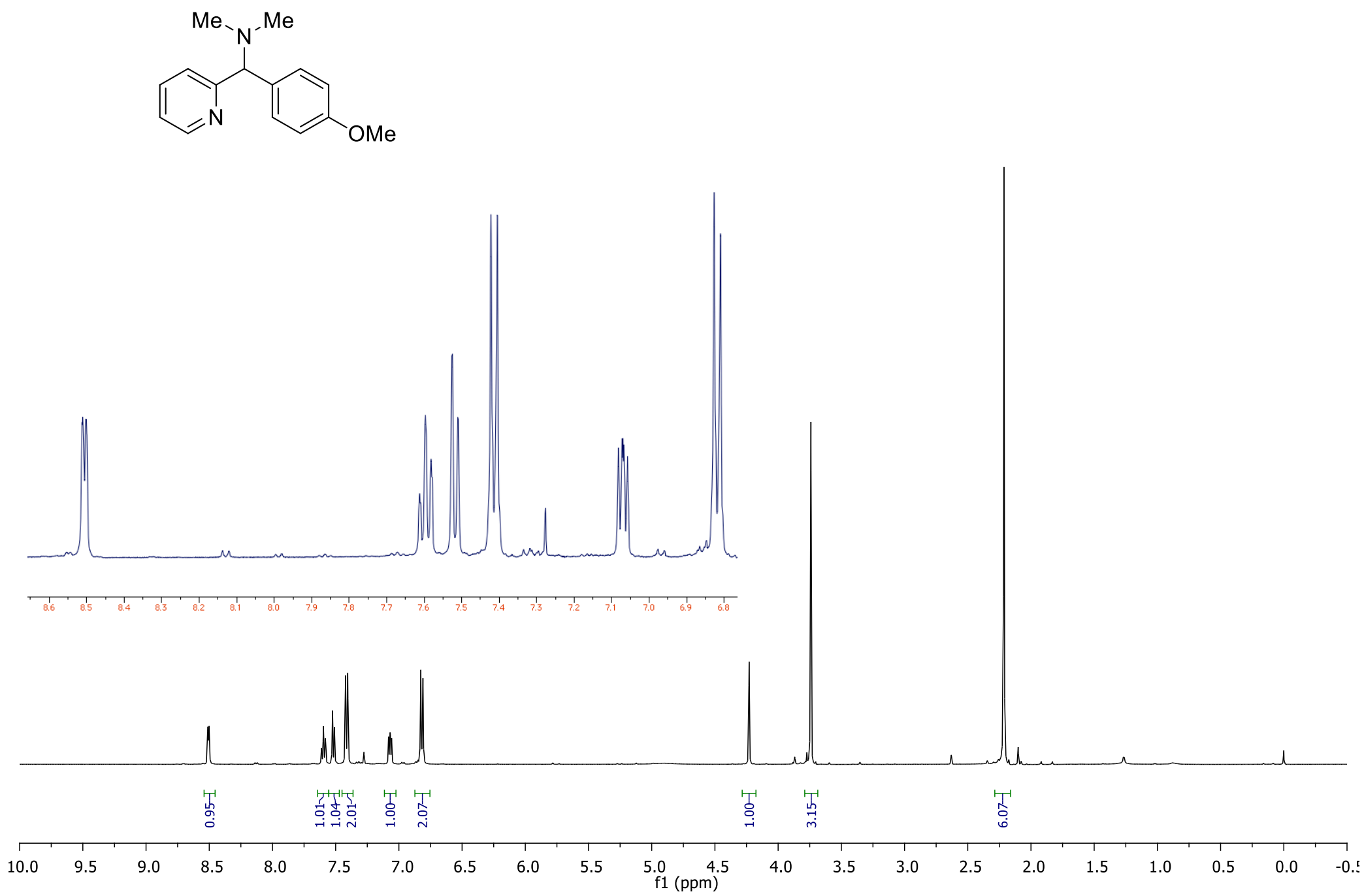
${ }^{13} \mathrm{C}$ NMR (125 MHz) spectrum of $\mathbf{4 d}$.
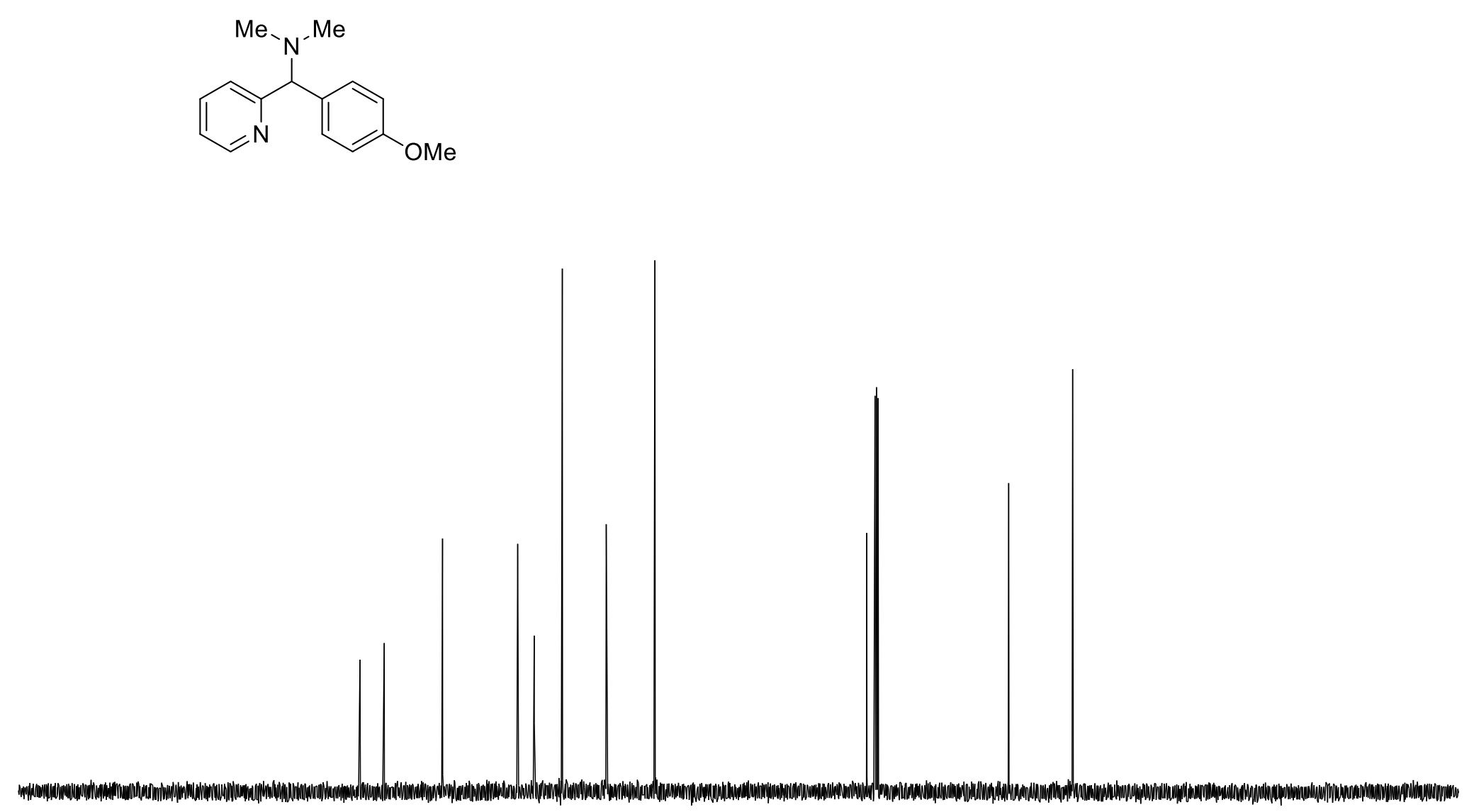

$\begin{array}{lllllllllll}210 & 200 & 190 & 180 & 170 & 160 & 150 & 140 & 130 & 120 & 110 \\ \mathrm{f} 1(\mathrm{ppm}) & 100\end{array}$ 
${ }^{1} \mathrm{H}$ NMR (500 MHz) spectrum of $\mathbf{4 e .}$

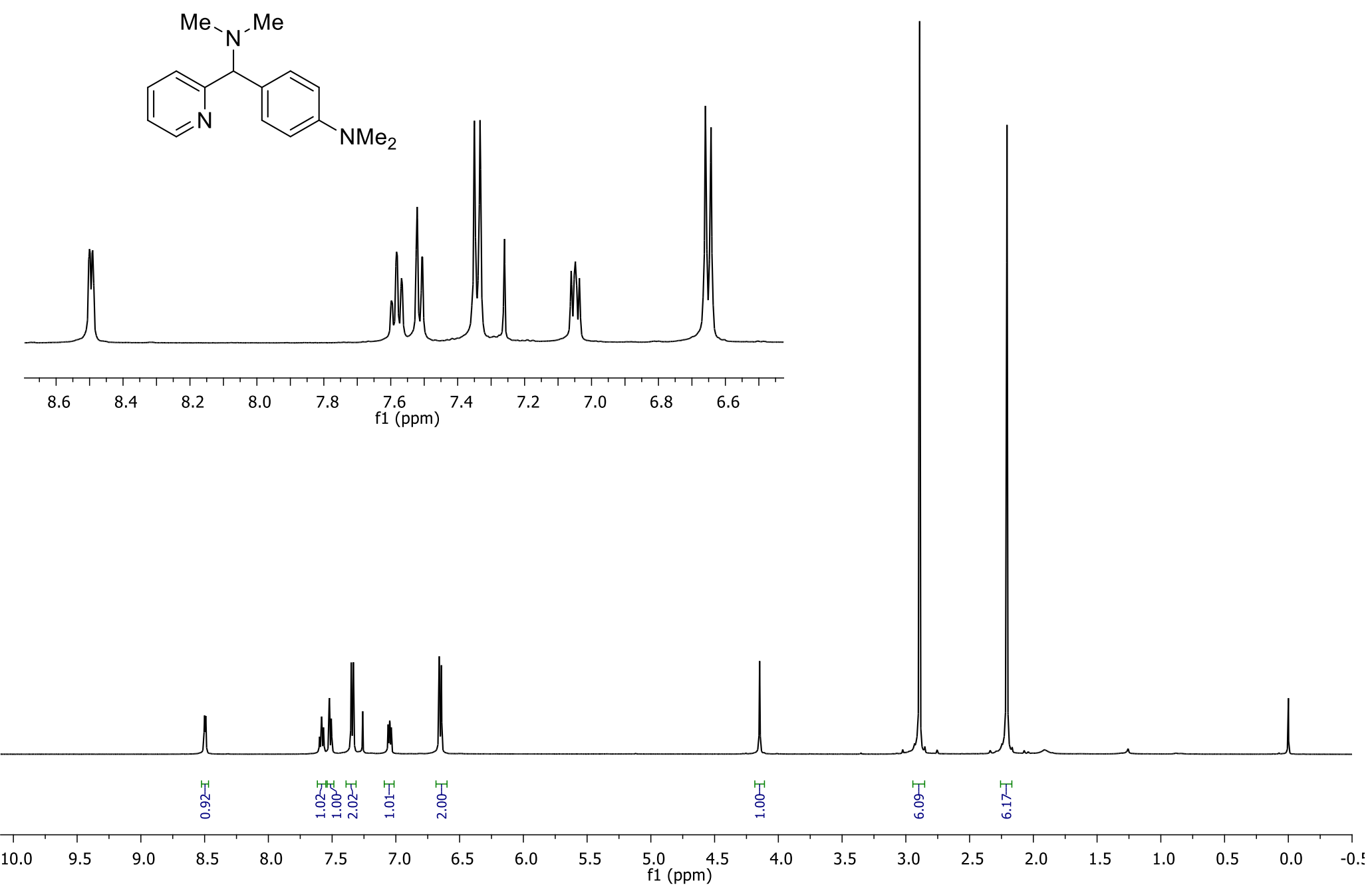


${ }^{13} \mathrm{C}$ NMR (125 MHz) spectrum of $4 \mathbf{e}$.<smiles>CN(C)c1ccc(C(c2ccccn2)N(C)C)cc1</smiles>
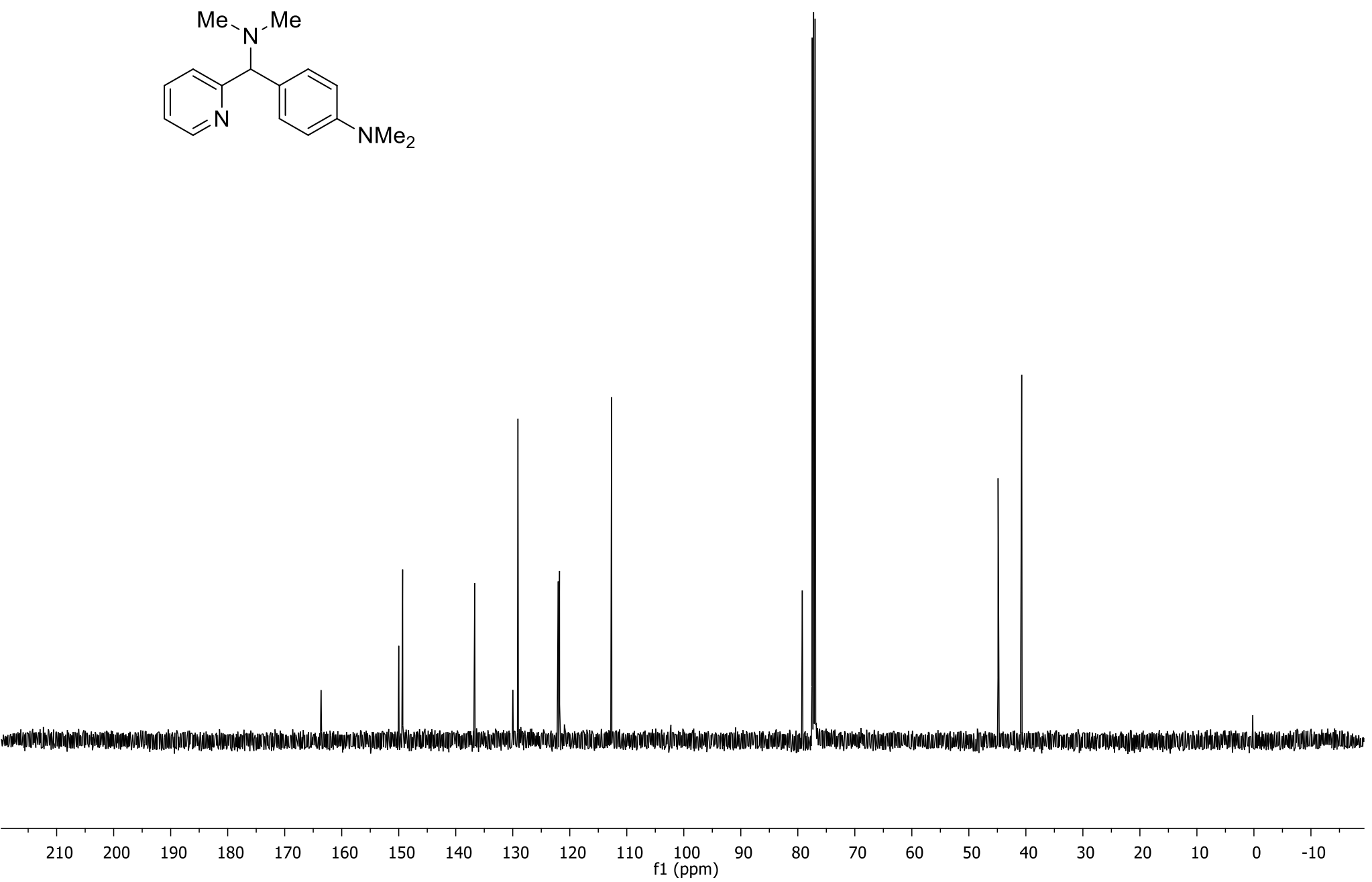

S 88 
${ }^{1} \mathrm{H}$ NMR (500 MHz) spectrum of $\mathbf{4 f}$.
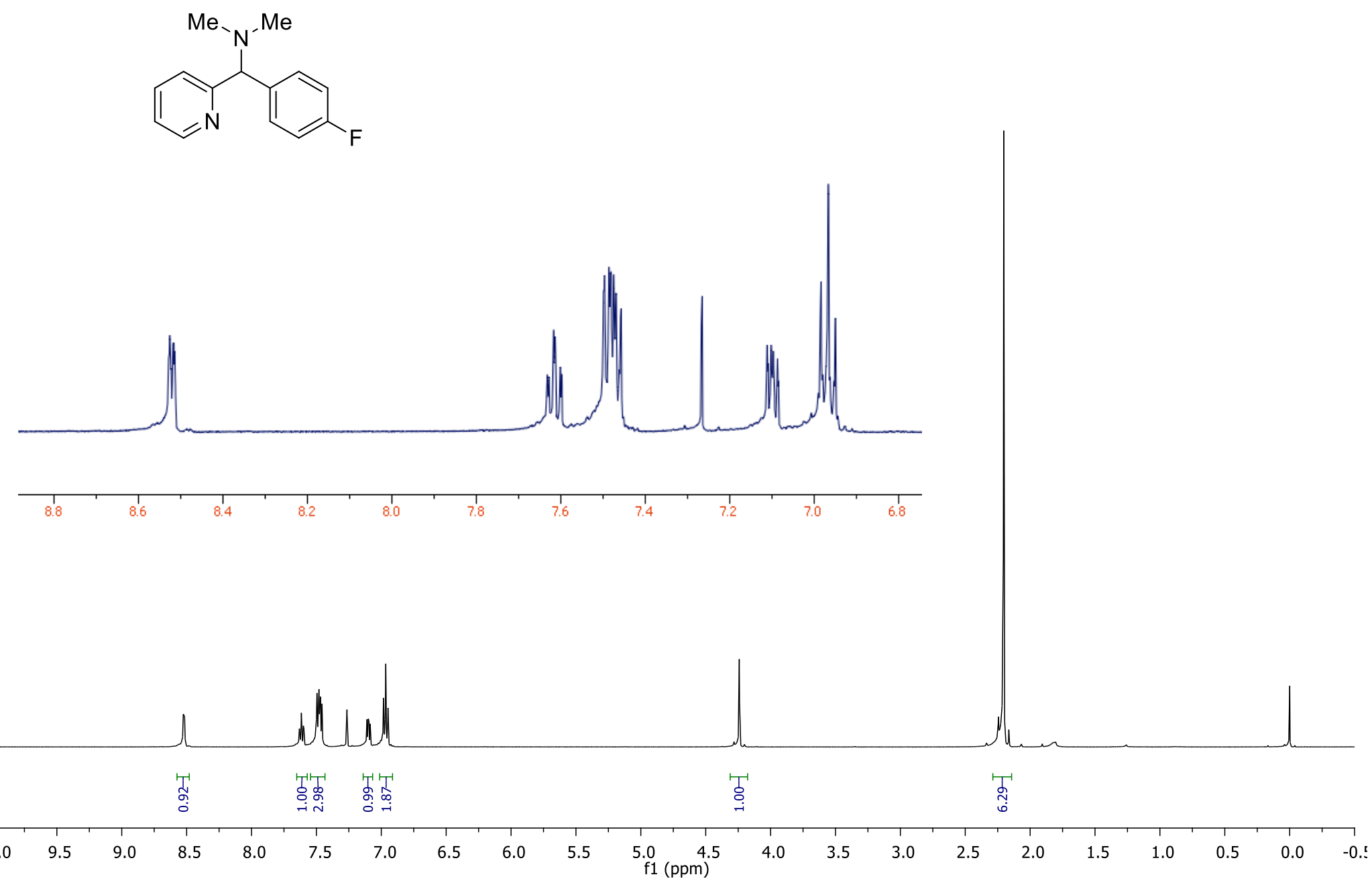
${ }^{13} \mathrm{C}$ NMR (125 MHz) spectrum of $\mathbf{4 f}$.
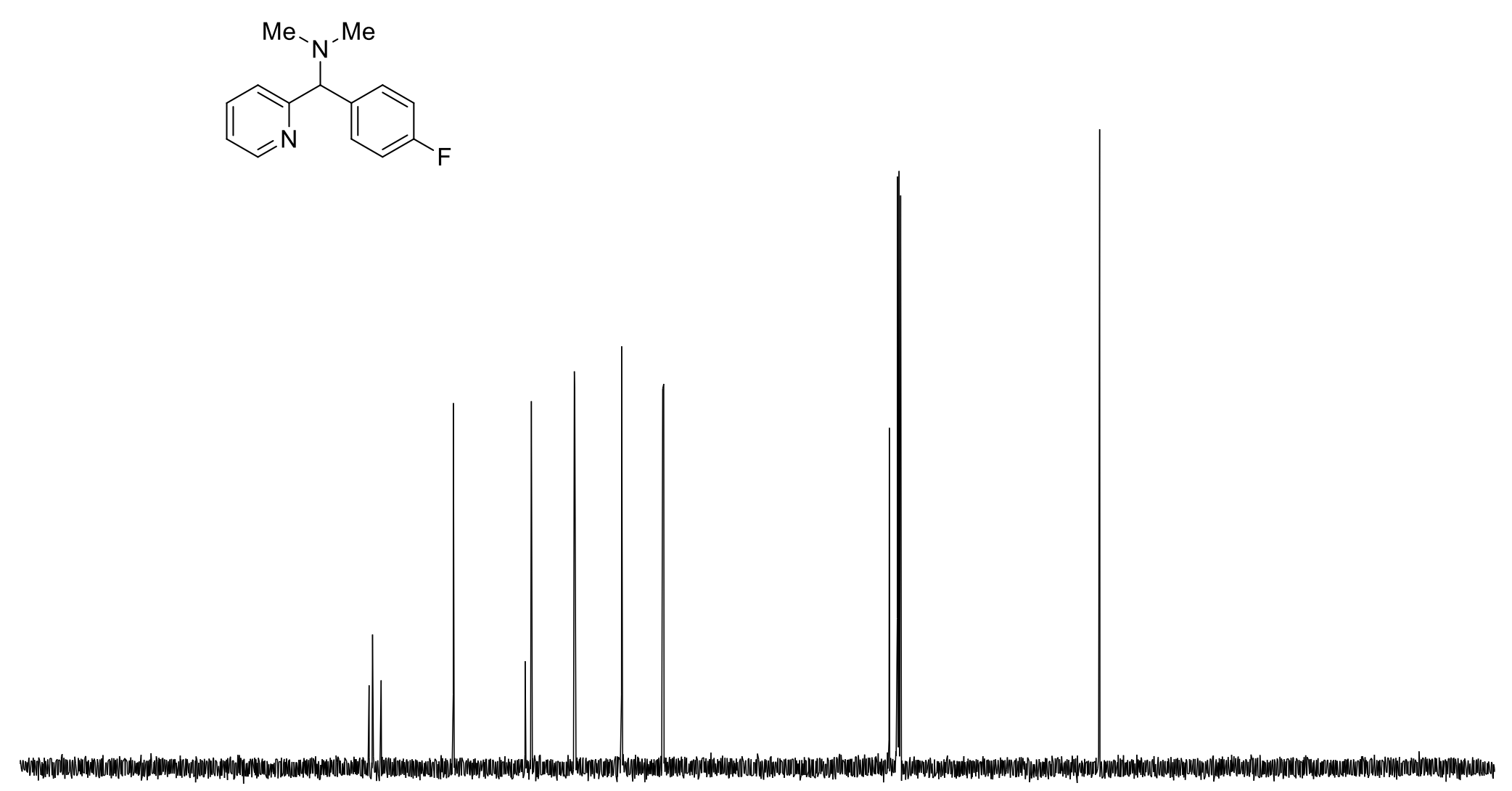

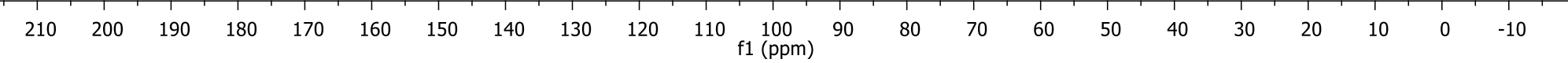




\section{${ }^{1} \mathrm{H}$ NMR (500 MHz) spectrum of $\mathbf{4 g}$.}

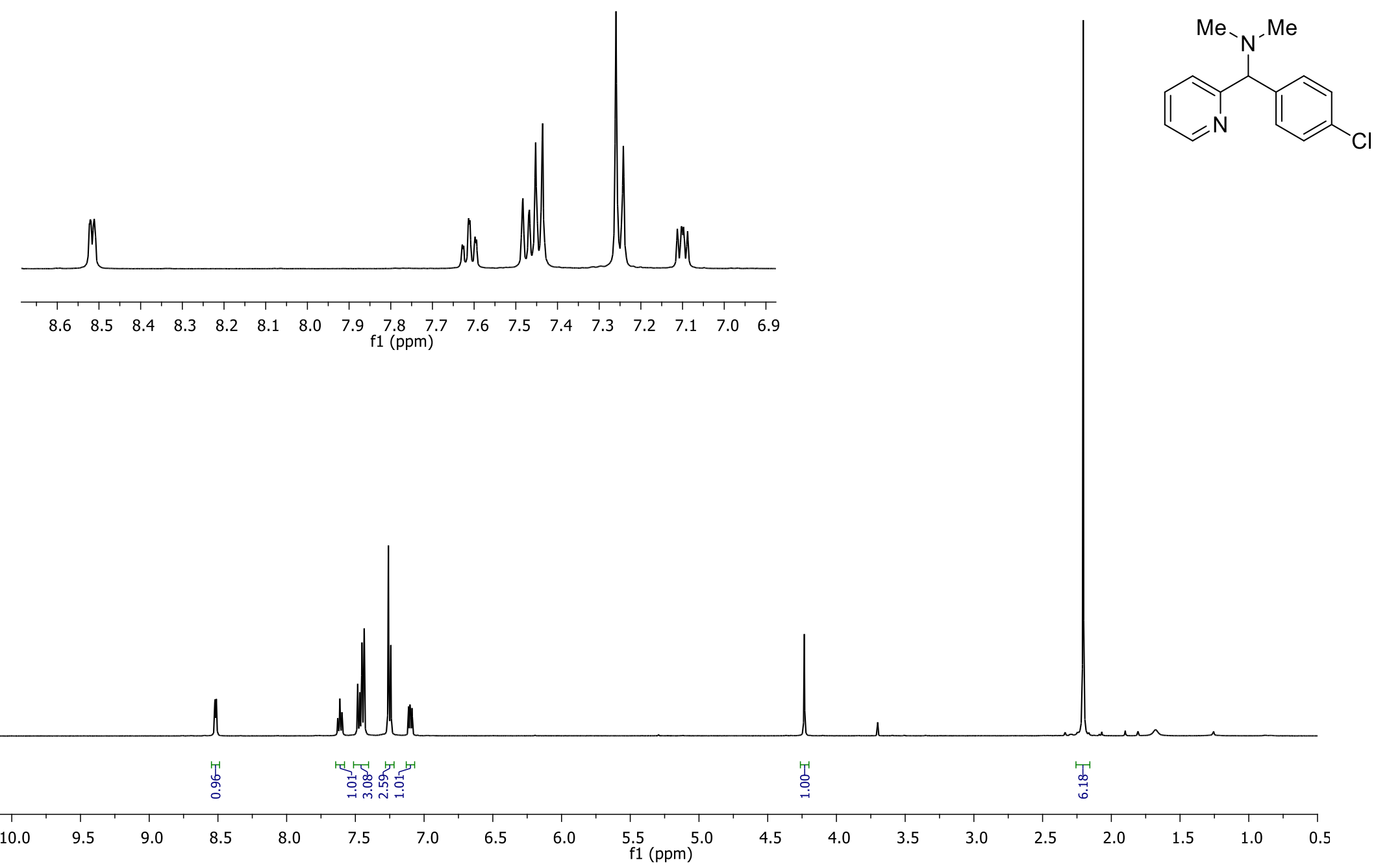


${ }^{13} \mathrm{C}$ NMR (125 MHz) spectrum of $\mathbf{4 g}$.
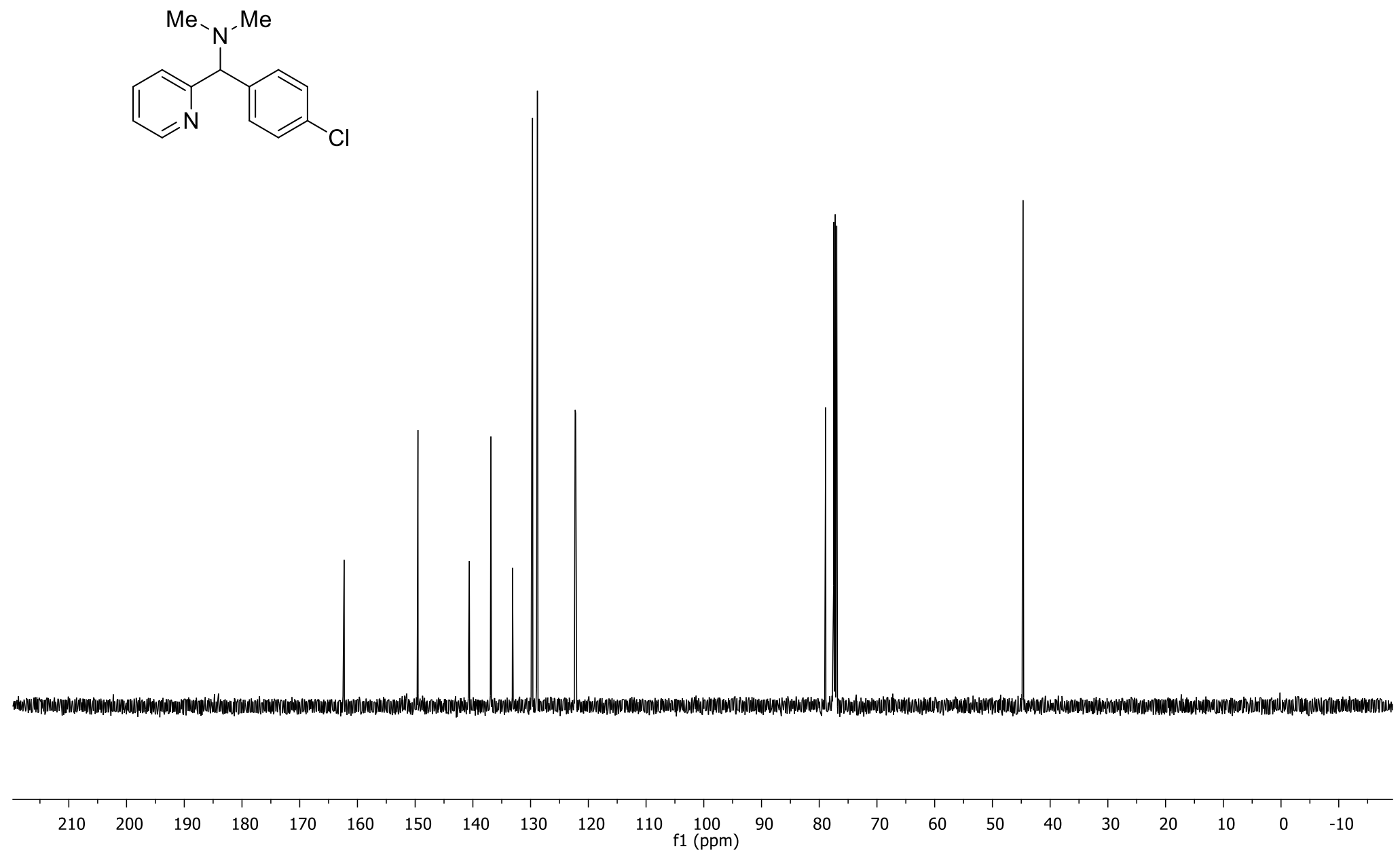

S 92 
${ }^{1} \mathrm{H}$ NMR (500 MHz) spectrum of $\mathbf{4 h}$.
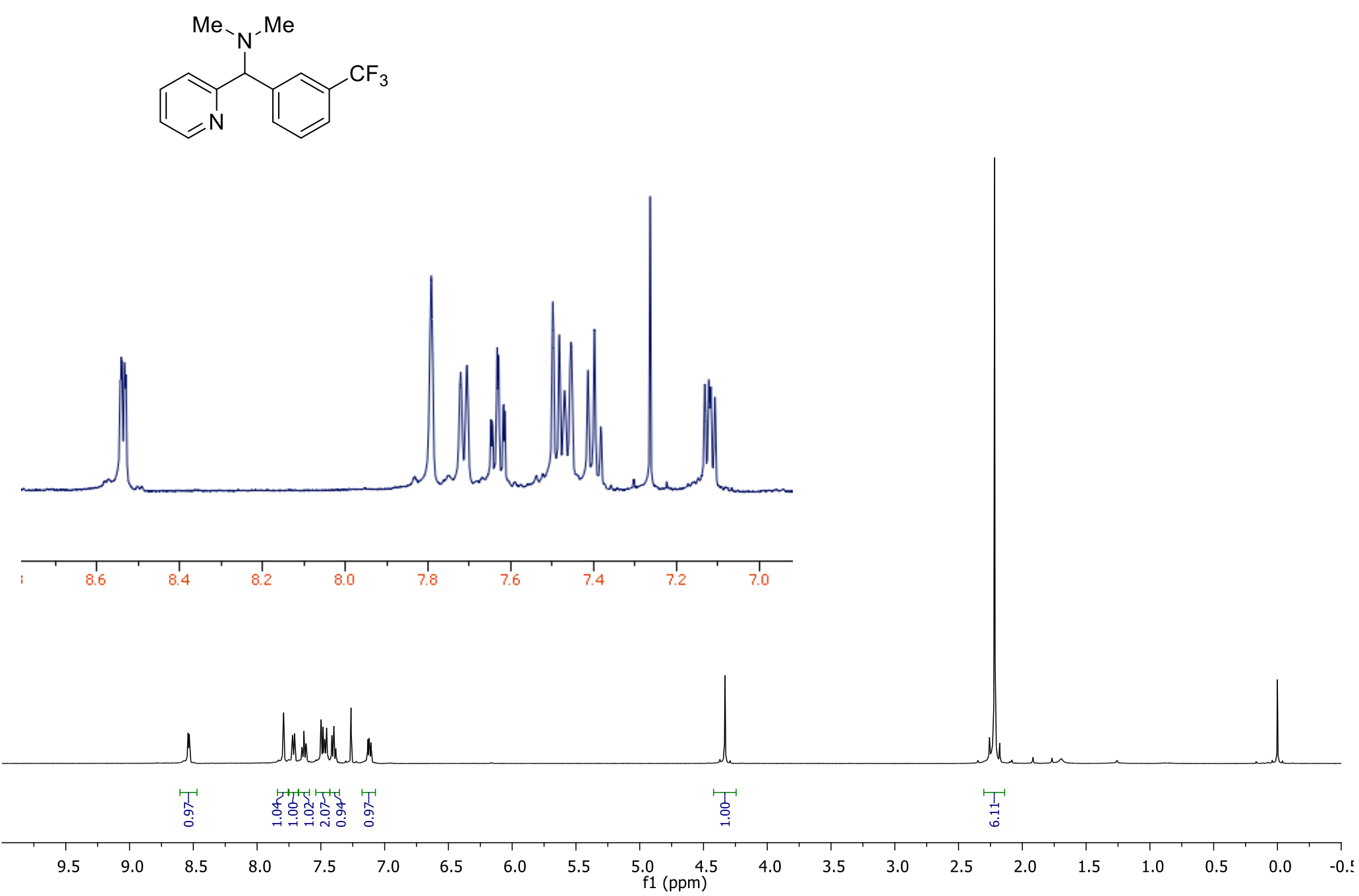
${ }^{13} \mathrm{C}$ NMR (125 MHz) spectrum of $\mathbf{4 h}$.
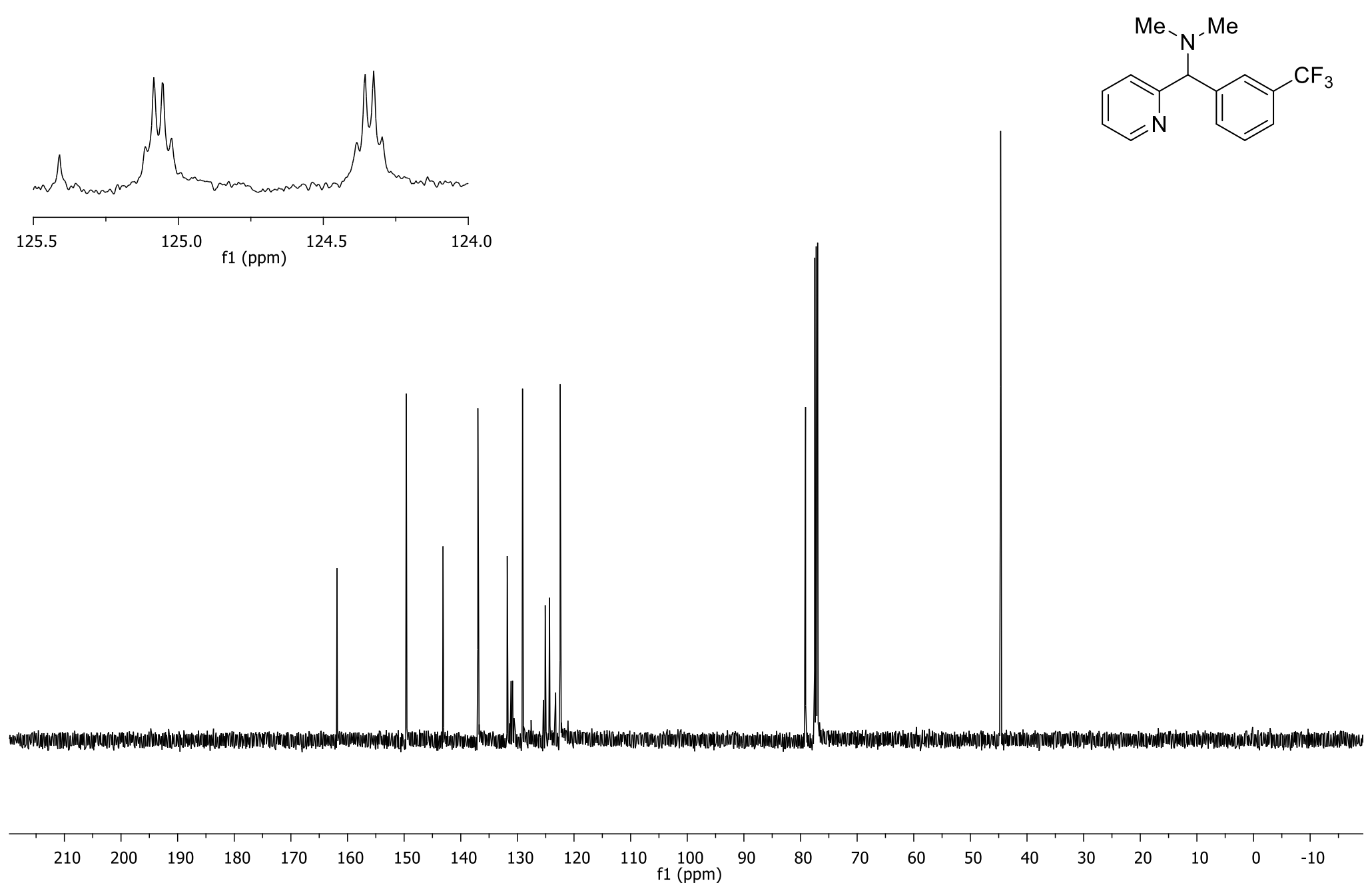
${ }^{1} \mathrm{H}$ NMR (500 MHz) spectrum of $\mathbf{4 i}$.<smiles>CN(C)C(c1ccc(C(F)(F)F)cc1)c1ccccn1</smiles>
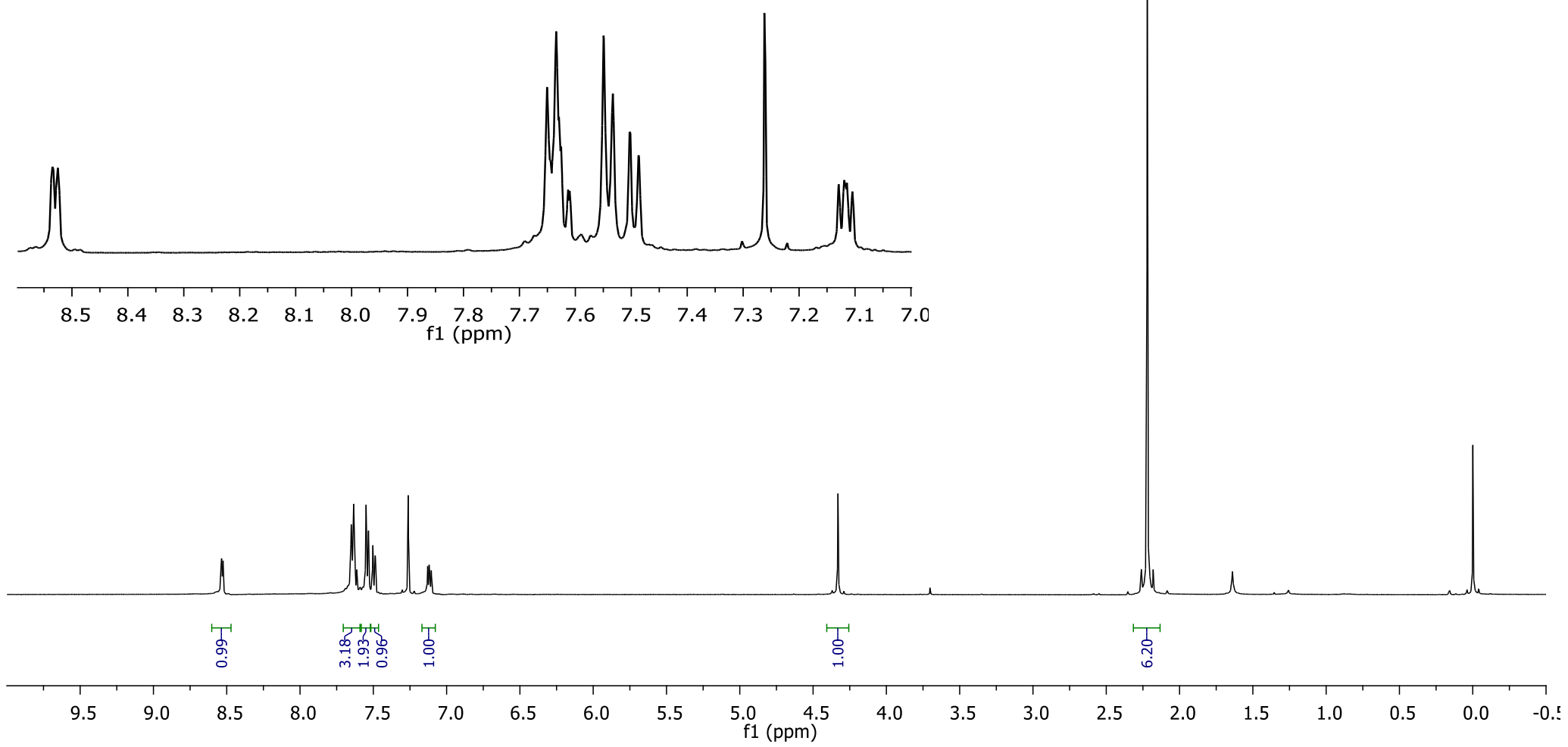

S 95 
${ }^{13} \mathrm{C}$ NMR (125 MHz) spectrum of $\mathbf{4 i}$.

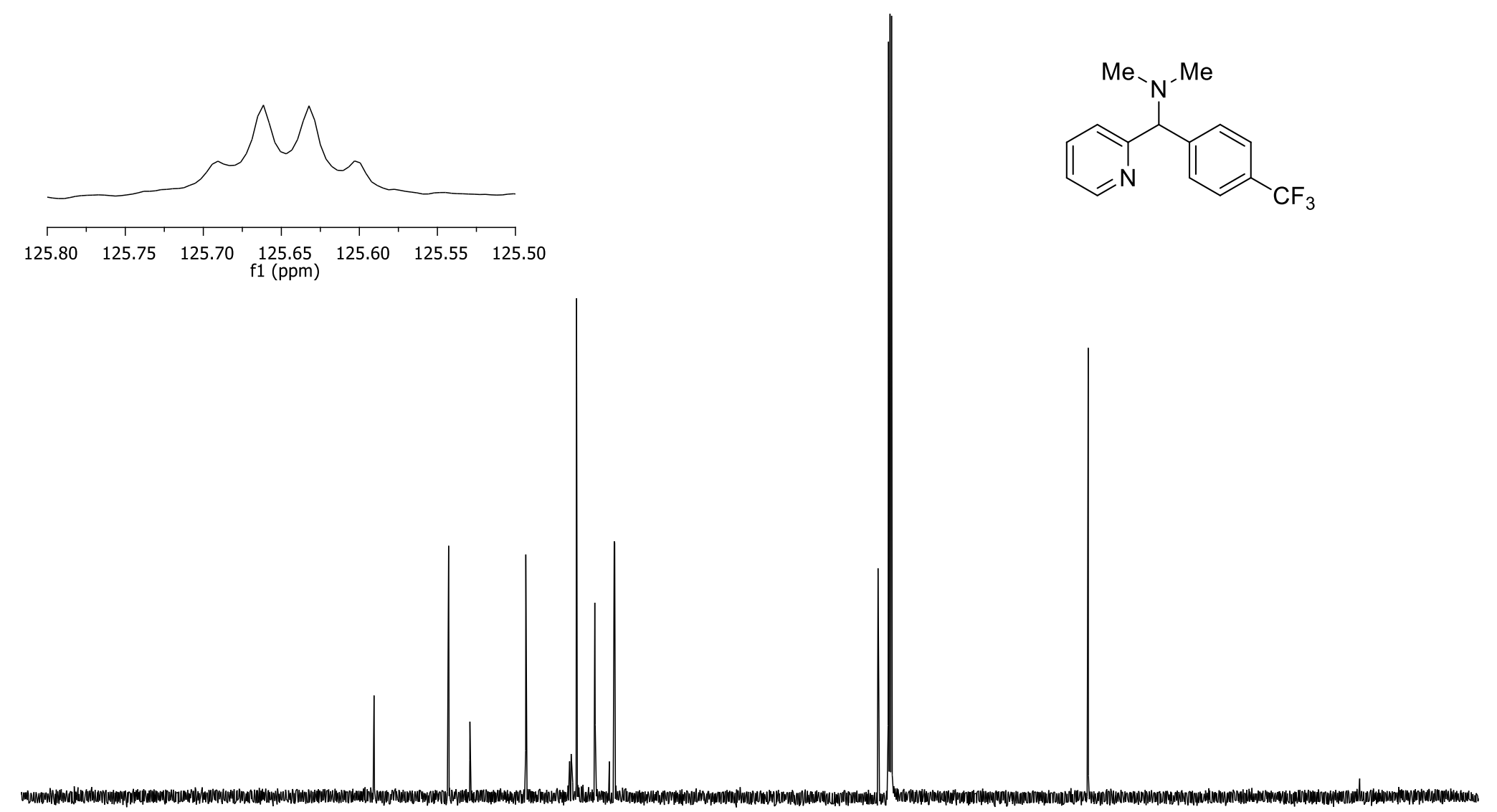

$\begin{array}{llllllllllllllllllllllll}210 & 200 & 190 & 180 & 170 & 160 & 150 & 140 & 130 & 120 & 110 & \begin{array}{c}100 \\ \mathrm{f} 1(\mathrm{ppm})\end{array} & 90 & 80 & 70 & 60 & 50 & 40 & 30 & 20 & 10 & 0 & -10\end{array}$ 
${ }^{1} \mathrm{H}$ NMR (500 MHz) spectrum of $\mathbf{4 j}$.

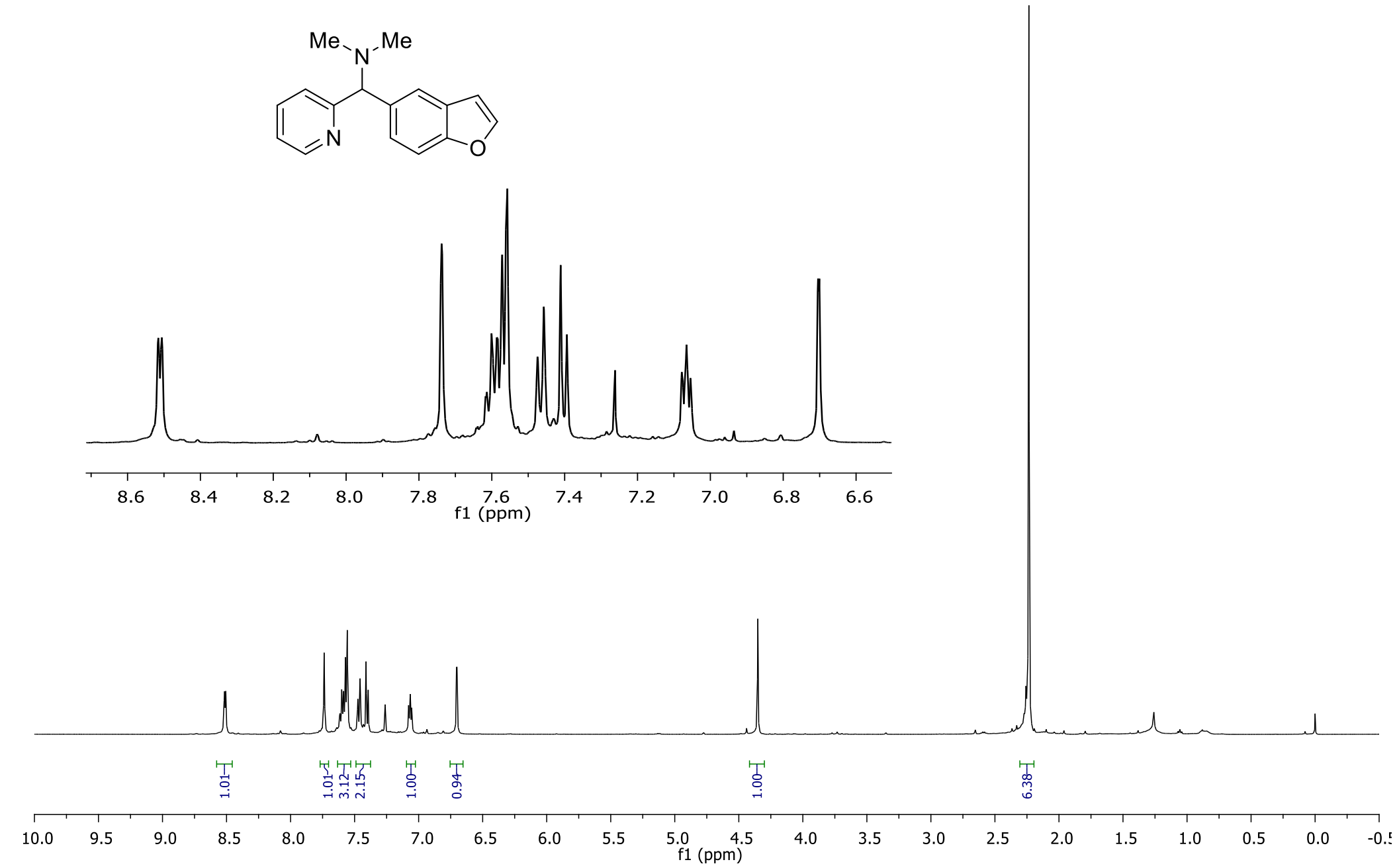


${ }^{13} \mathrm{C}$ NMR (125 MHz) spectrum of $\mathbf{4 j}$.

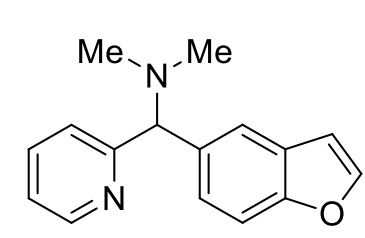

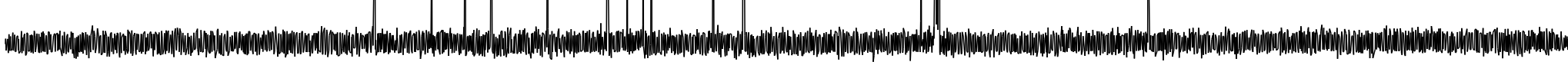

$\begin{array}{llllllllllllllllllllllllll} & 210 & 200 & 190 & 180 & 170 & 160 & 150 & 140 & 130 & 120 & 110 & \underset{f 1}{100}(\mathrm{ppm}) & 90 & 80 & 70 & 60 & 50 & 40 & 30 & 20 & 10 & 0 & -10\end{array}$


${ }^{1} \mathrm{H}$ NMR (500 MHz) spectrum of $\mathbf{4 k}$.

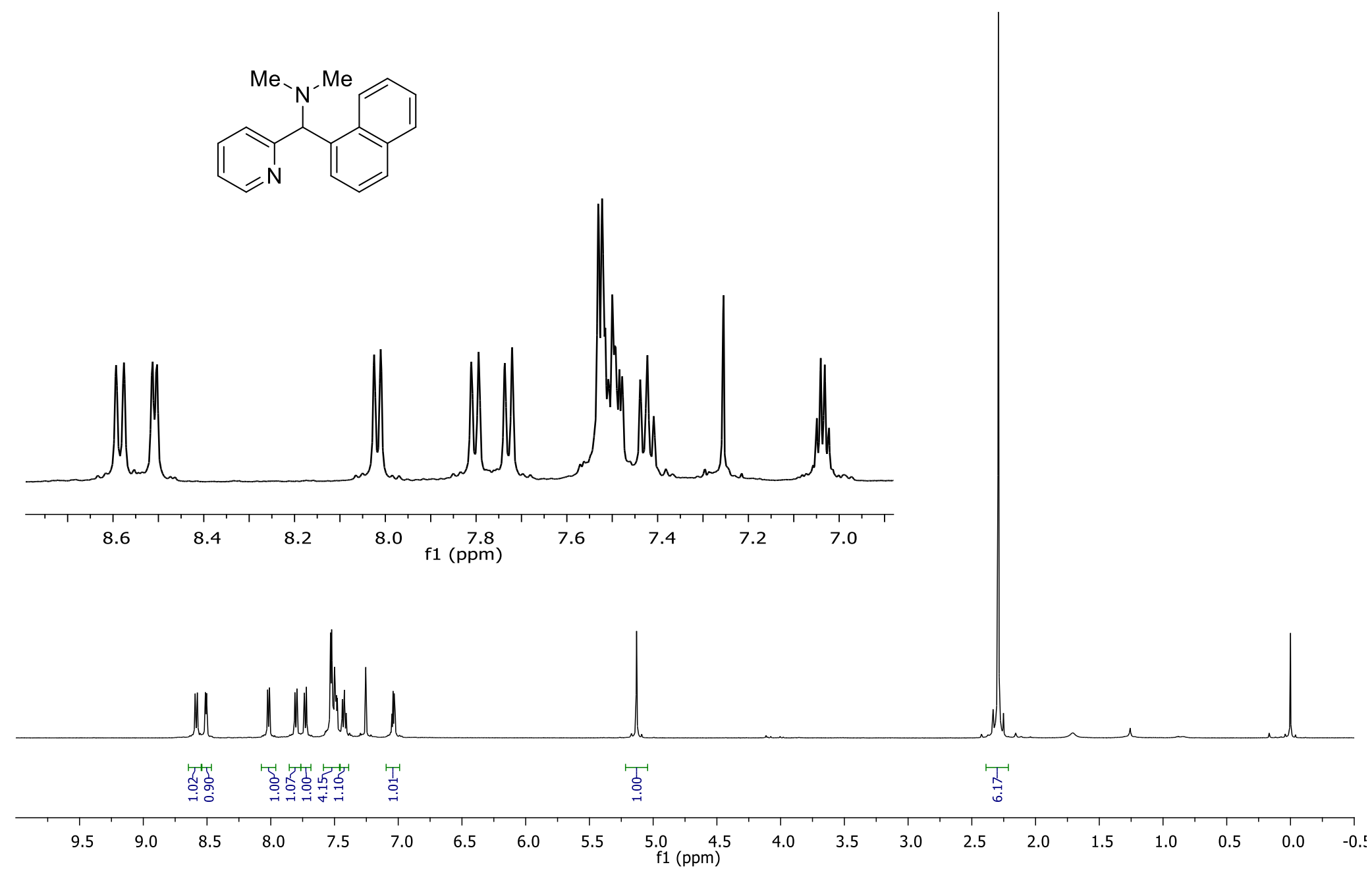


${ }^{13} \mathrm{C}$ NMR (125 MHz) spectrum of $\mathbf{4 k}$.
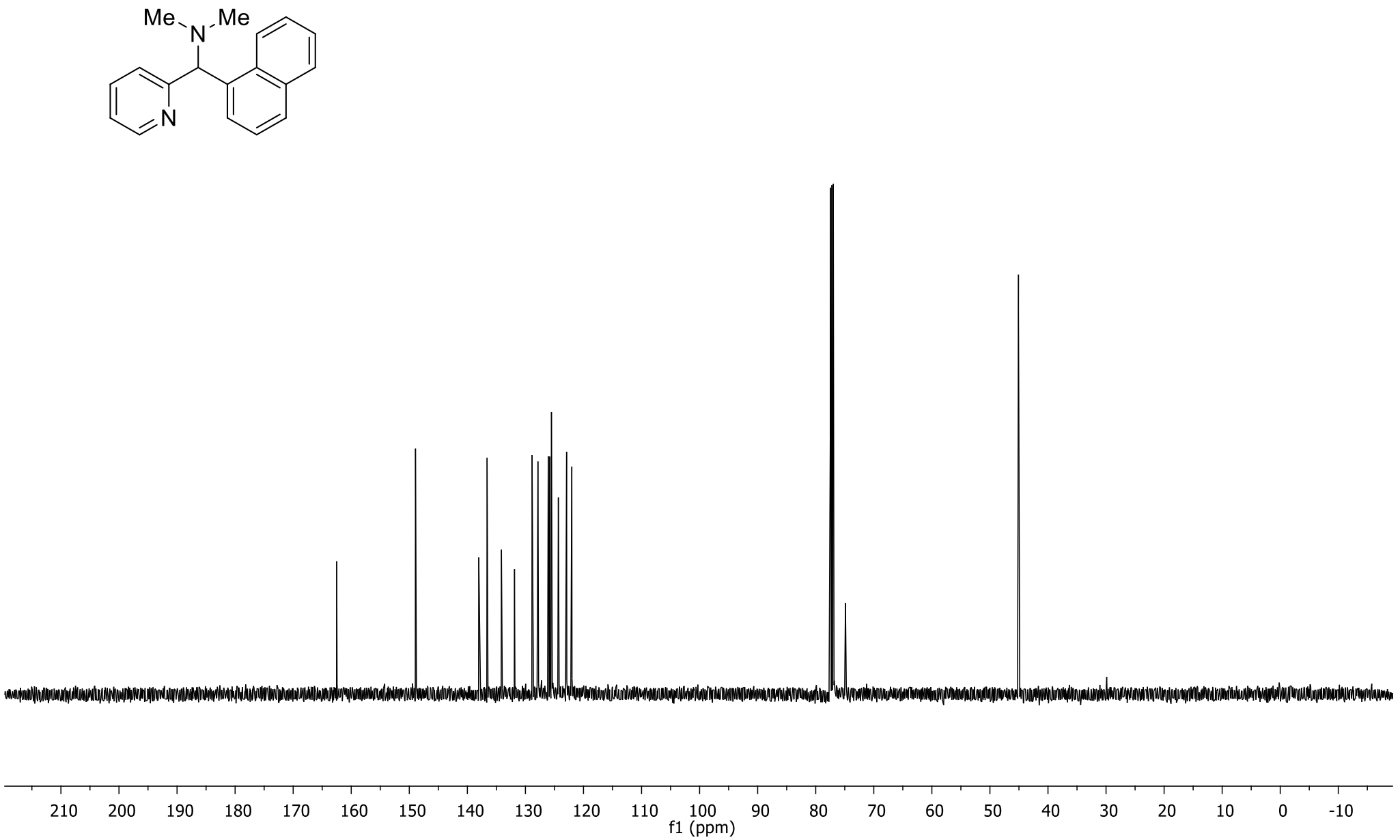

S 100 
${ }^{1} \mathrm{H}$ NMR (500 MHz) spectrum of $\mathbf{4 l}$.
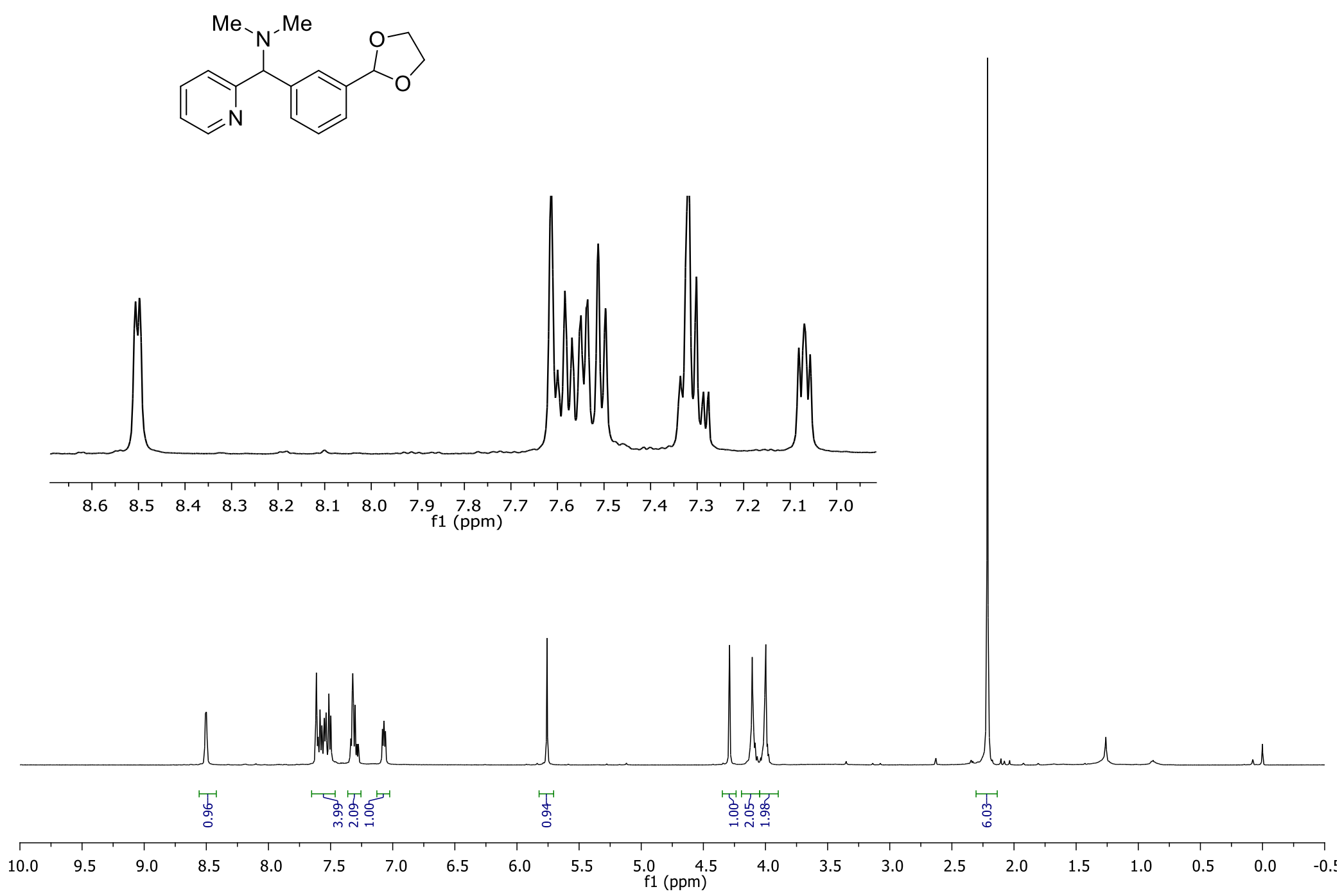


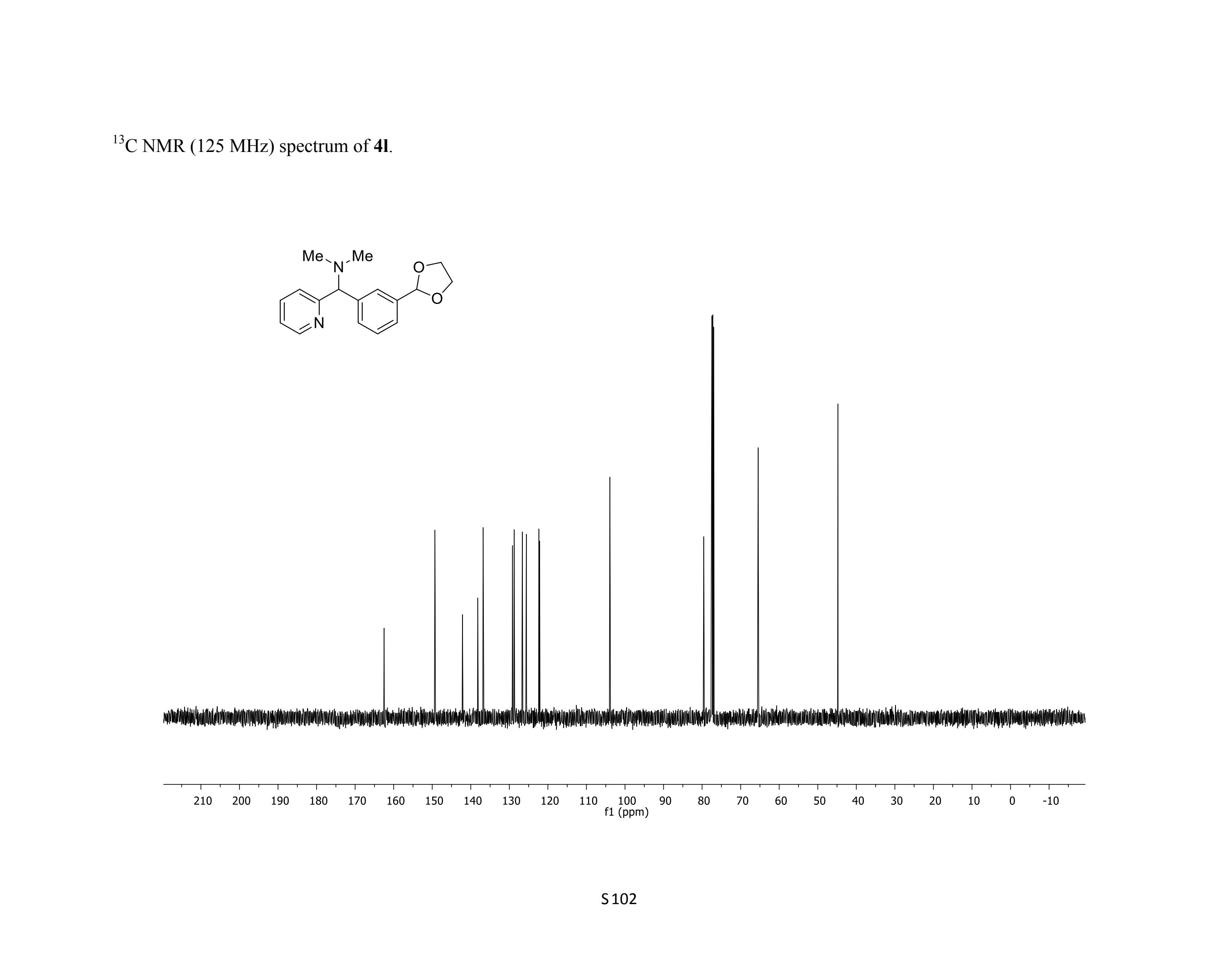


${ }^{1} \mathrm{H}$ NMR (500 MHz) spectrum of $\mathbf{4 m}$.

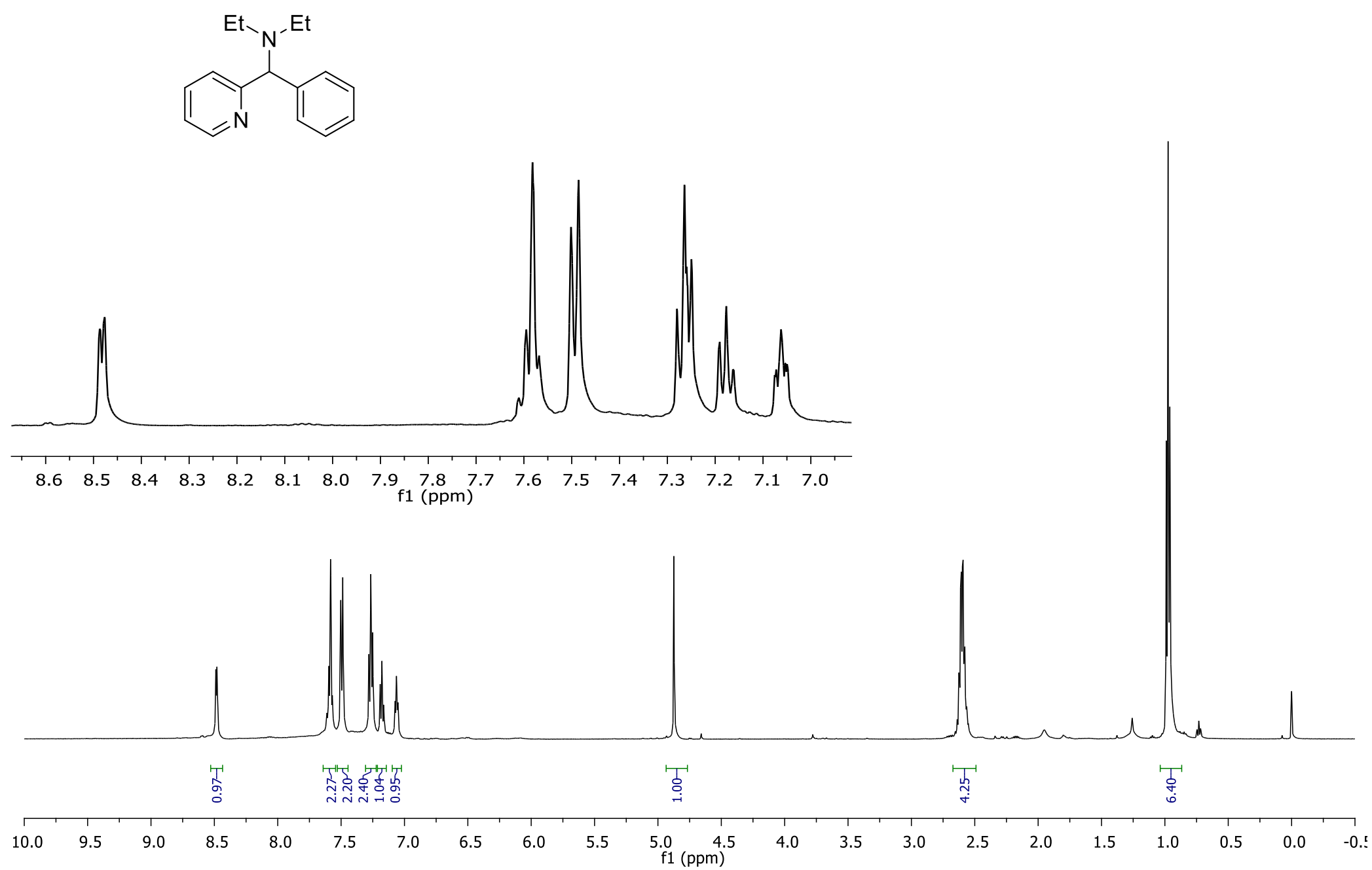




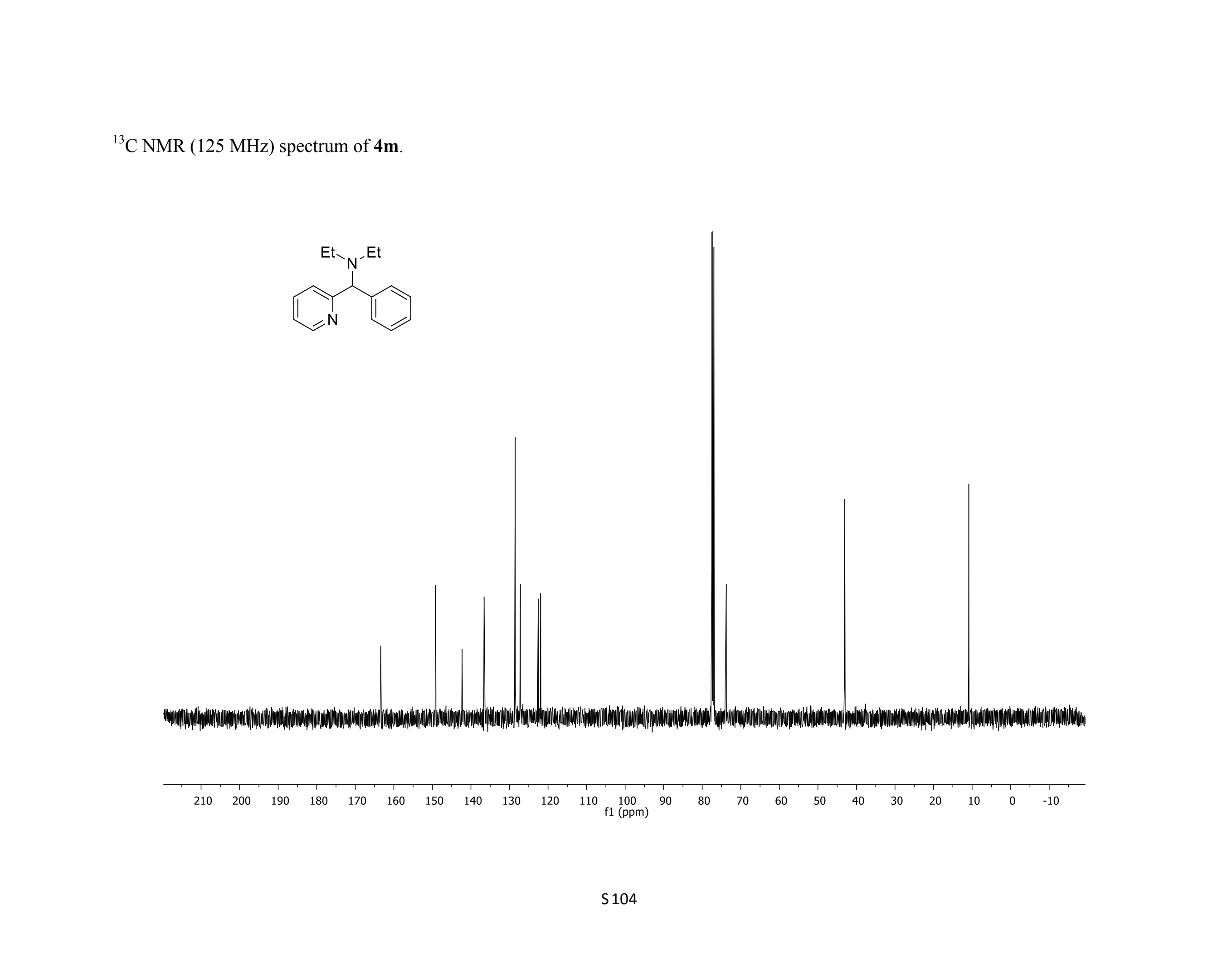


${ }^{1} \mathrm{H}$ NMR (500 MHz) spectrum of $\mathbf{4 n}$.
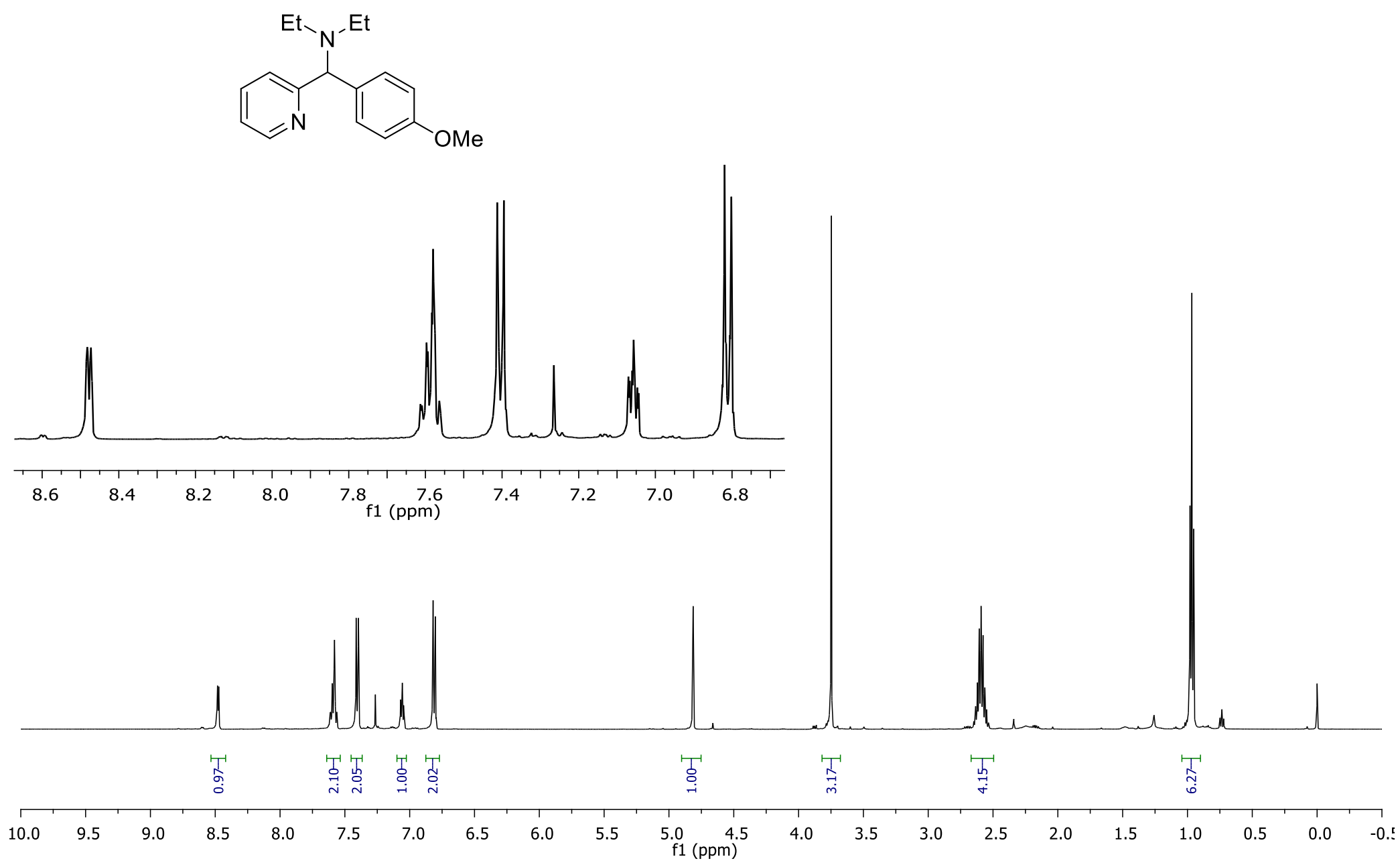
${ }^{13} \mathrm{C}$ NMR (125 MHz) spectrum of $\mathbf{4 n}$.

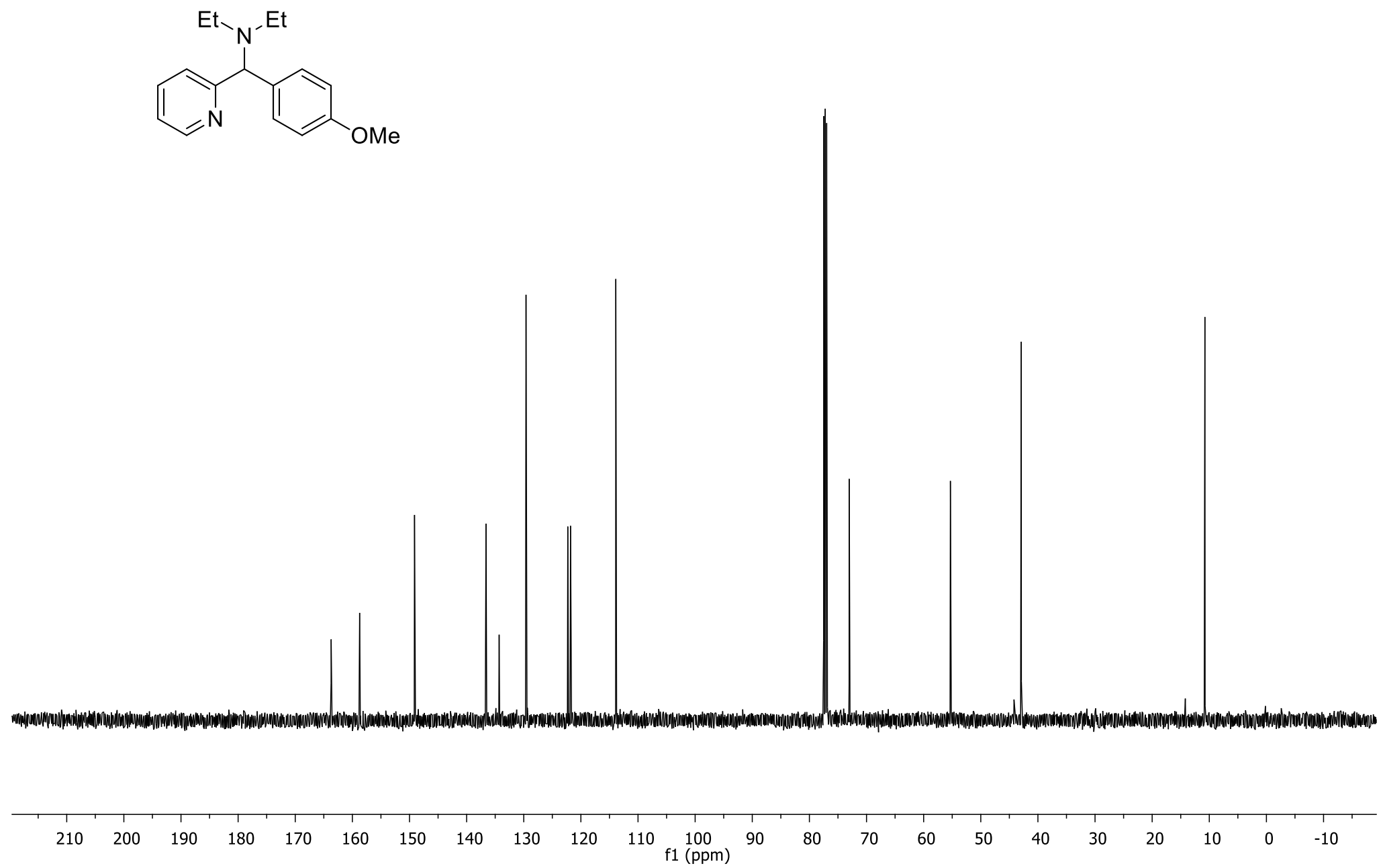

S106 
${ }^{1} \mathrm{H}$ NMR (500 MHz) spectrum of $4 \mathbf{0 .}$

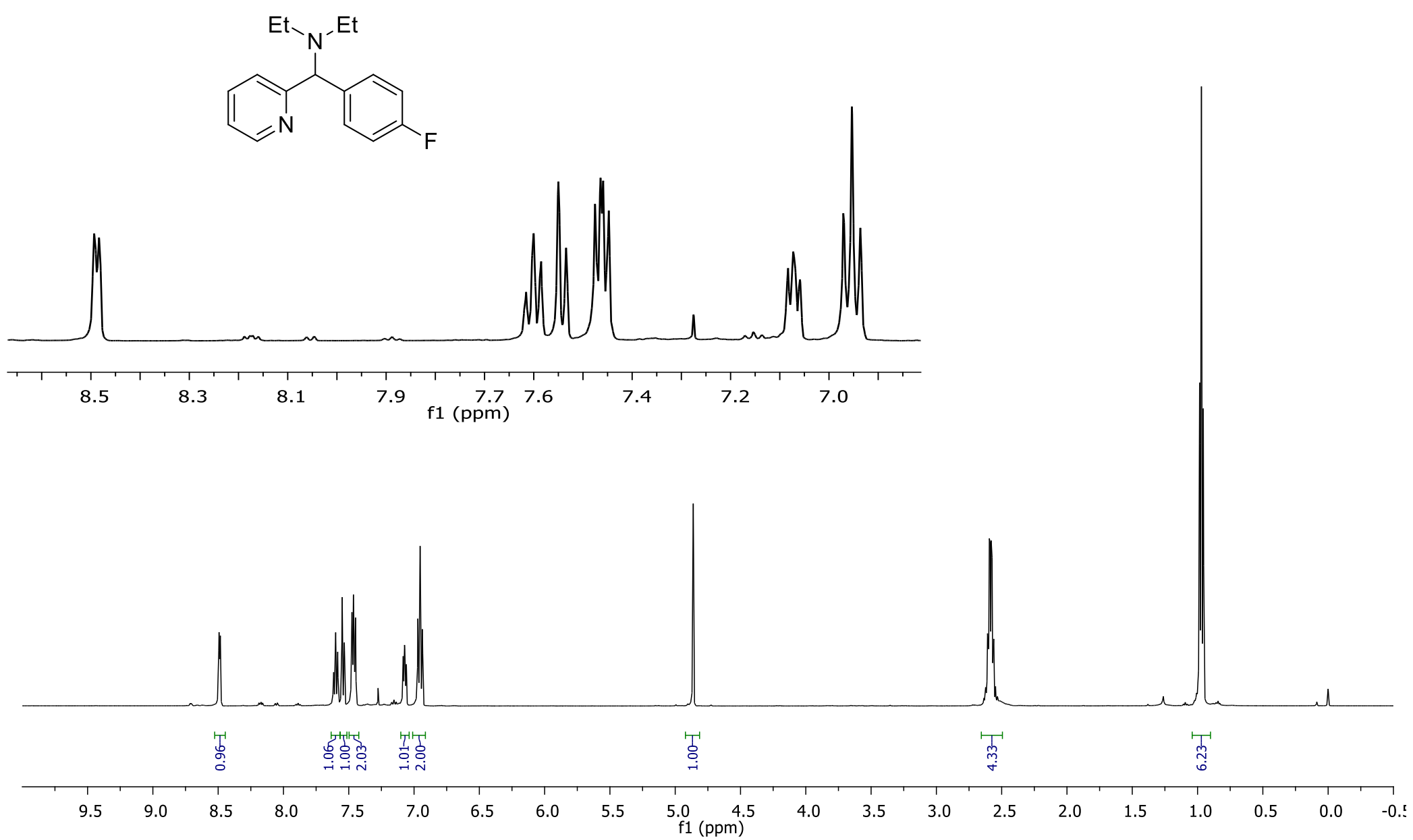


${ }^{13} \mathrm{C}$ NMR (125 MHz) spectrum of $4 \mathbf{o .}$
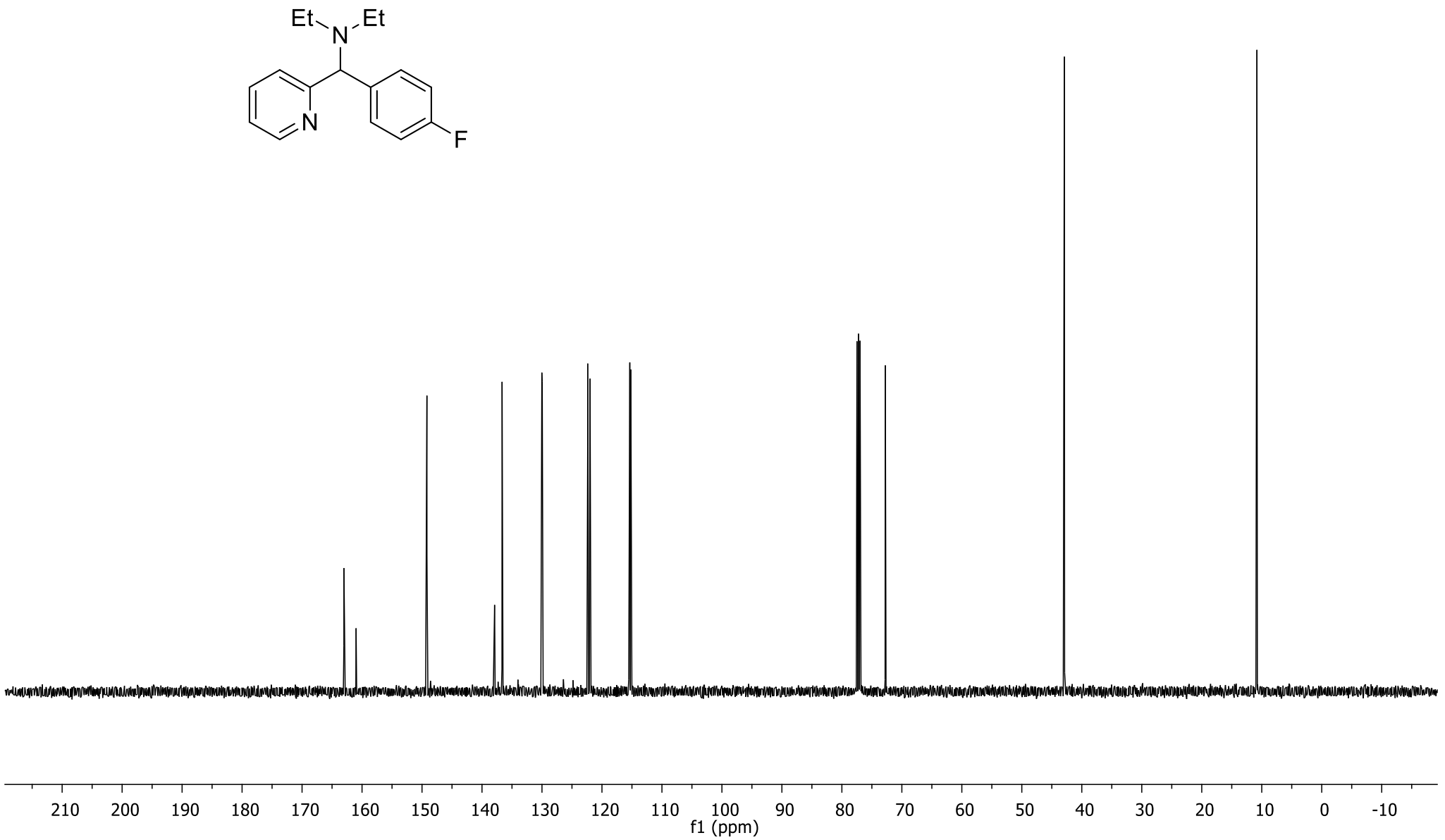

S108 
${ }^{1} \mathrm{H}$ NMR (500 MHz) spectrum of $\mathbf{5 a}$.
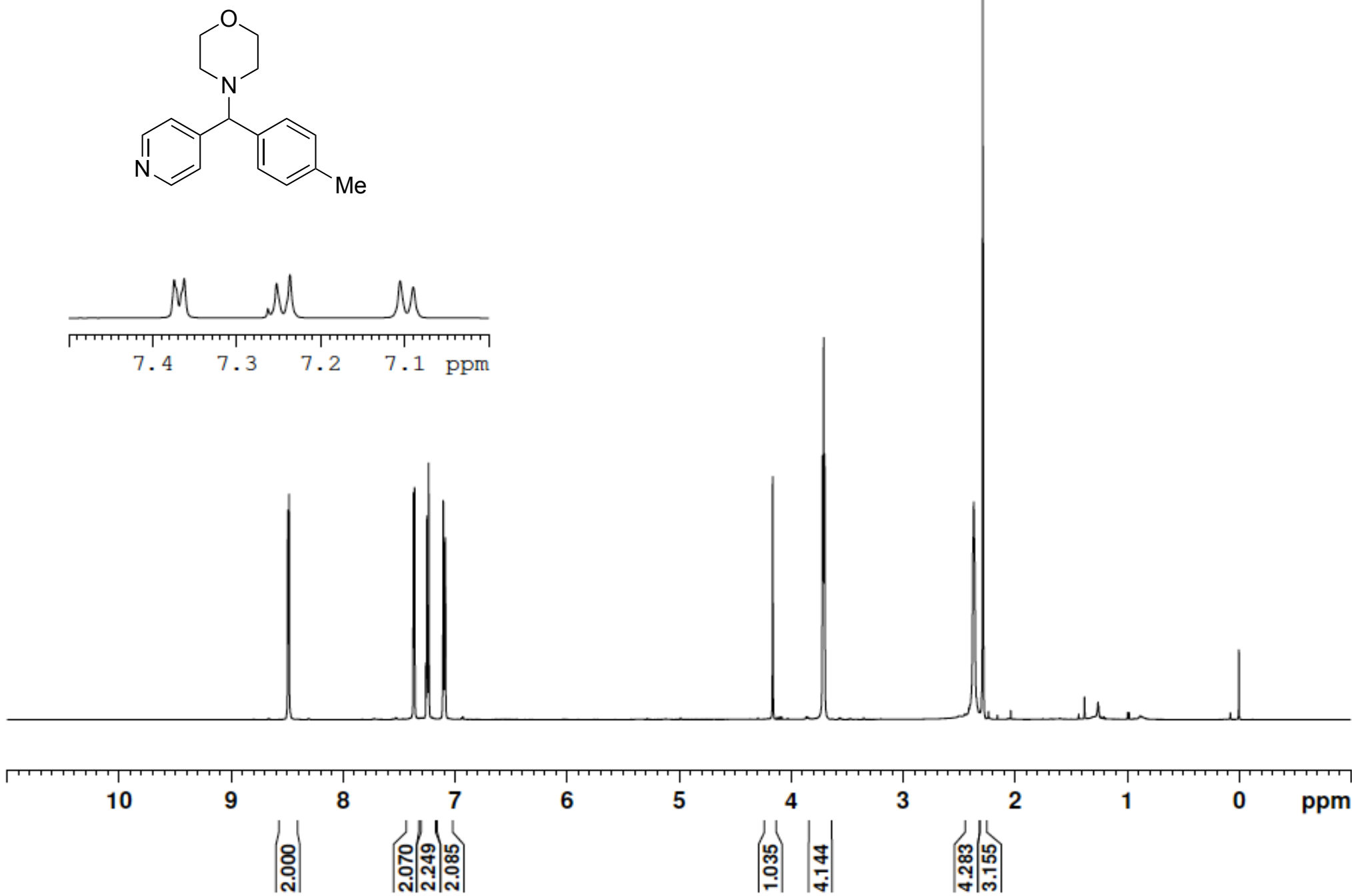
${ }^{13} \mathrm{C}$ NMR (125 MHz) spectrum of $\mathbf{5 a}$.

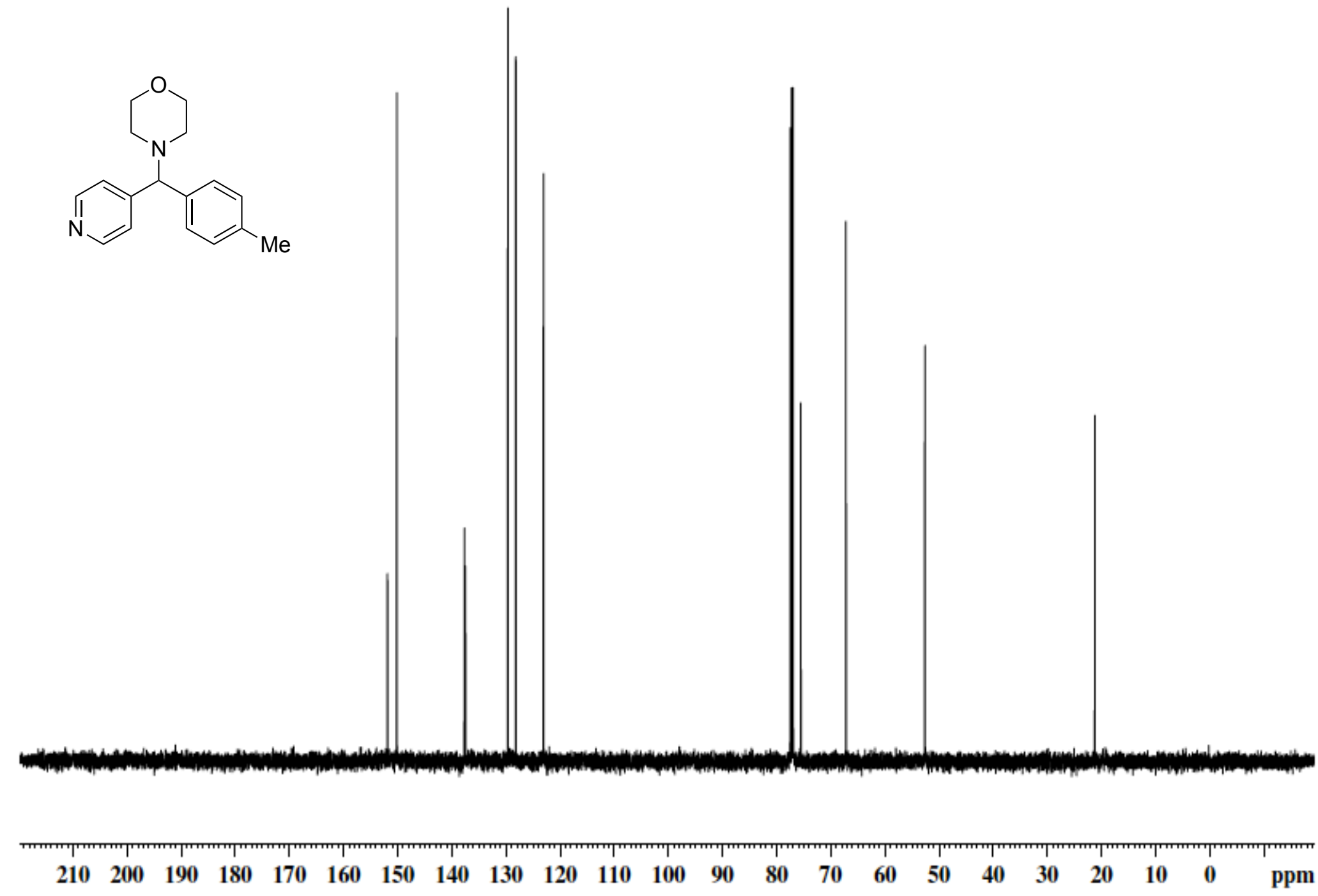


${ }^{1} \mathrm{H}$ NMR (500 MHz) spectrum of $\mathbf{5 b}$.
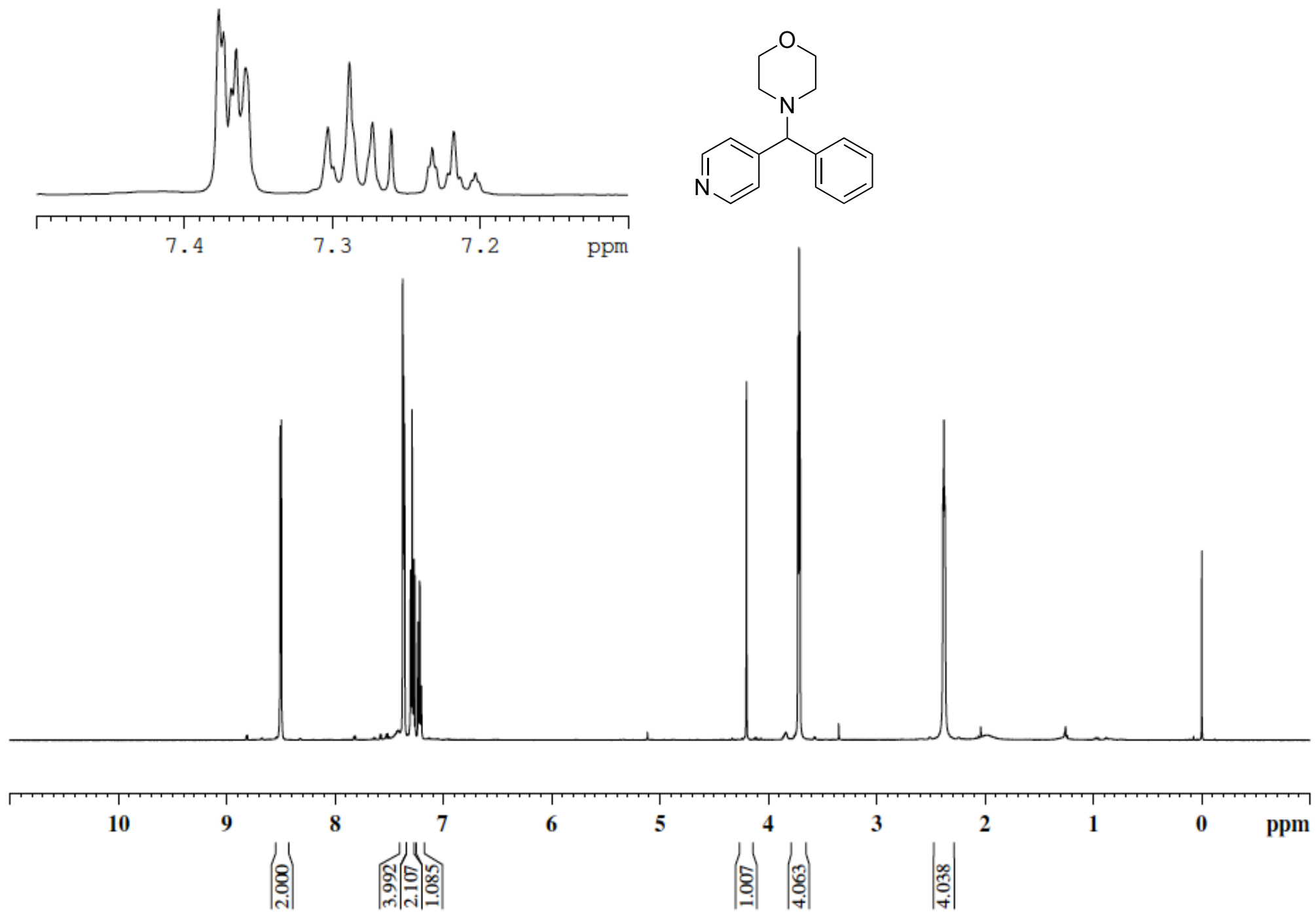
${ }^{13} \mathrm{C}$ NMR (125 MHz) spectrum of $\mathbf{5 b}$.

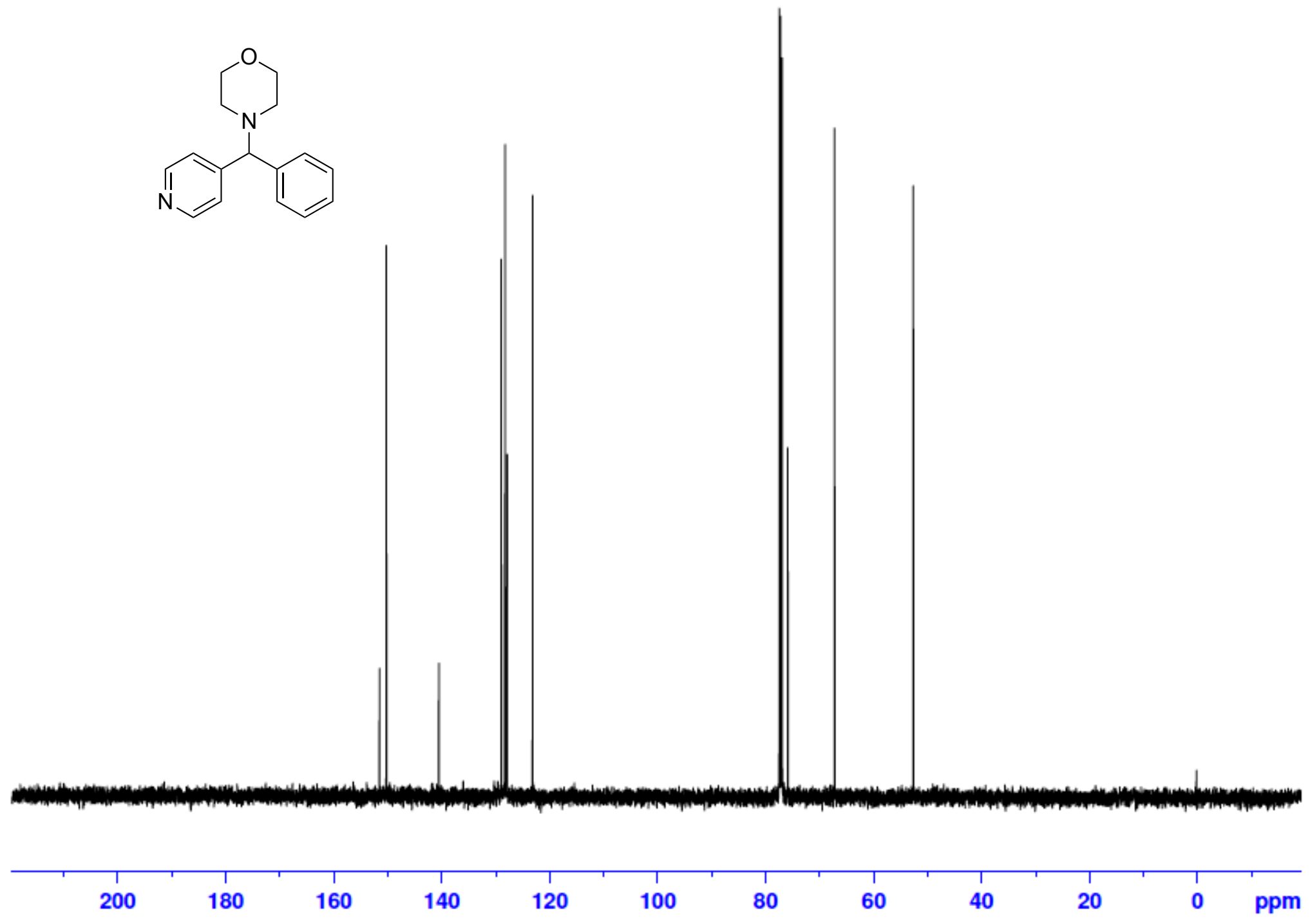


${ }^{1} \mathrm{H}$ NMR (500 MHz) spectrum of 5c.
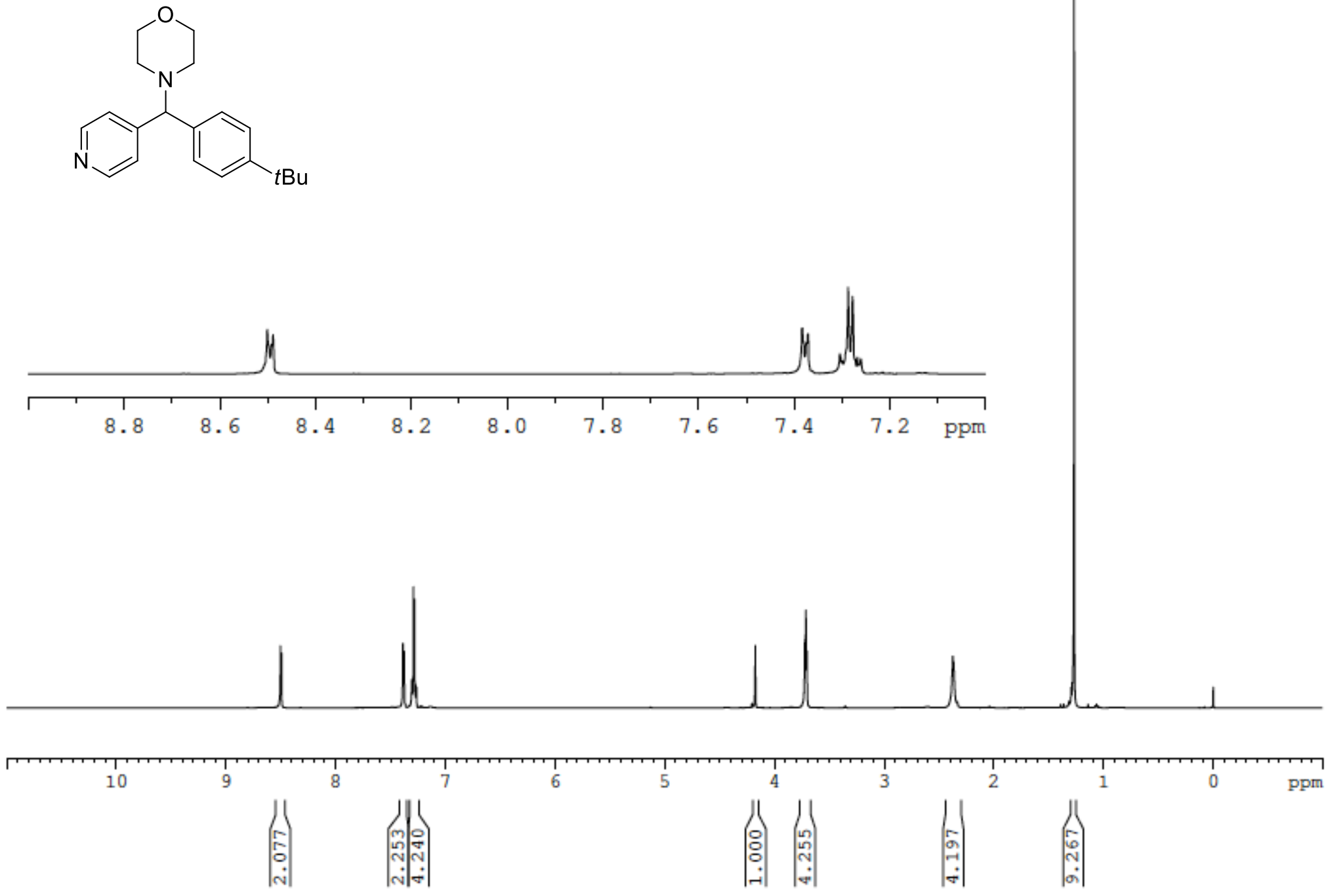
${ }^{13} \mathrm{C}$ NMR (125 MHz) spectrum of $\mathbf{5 c}$.
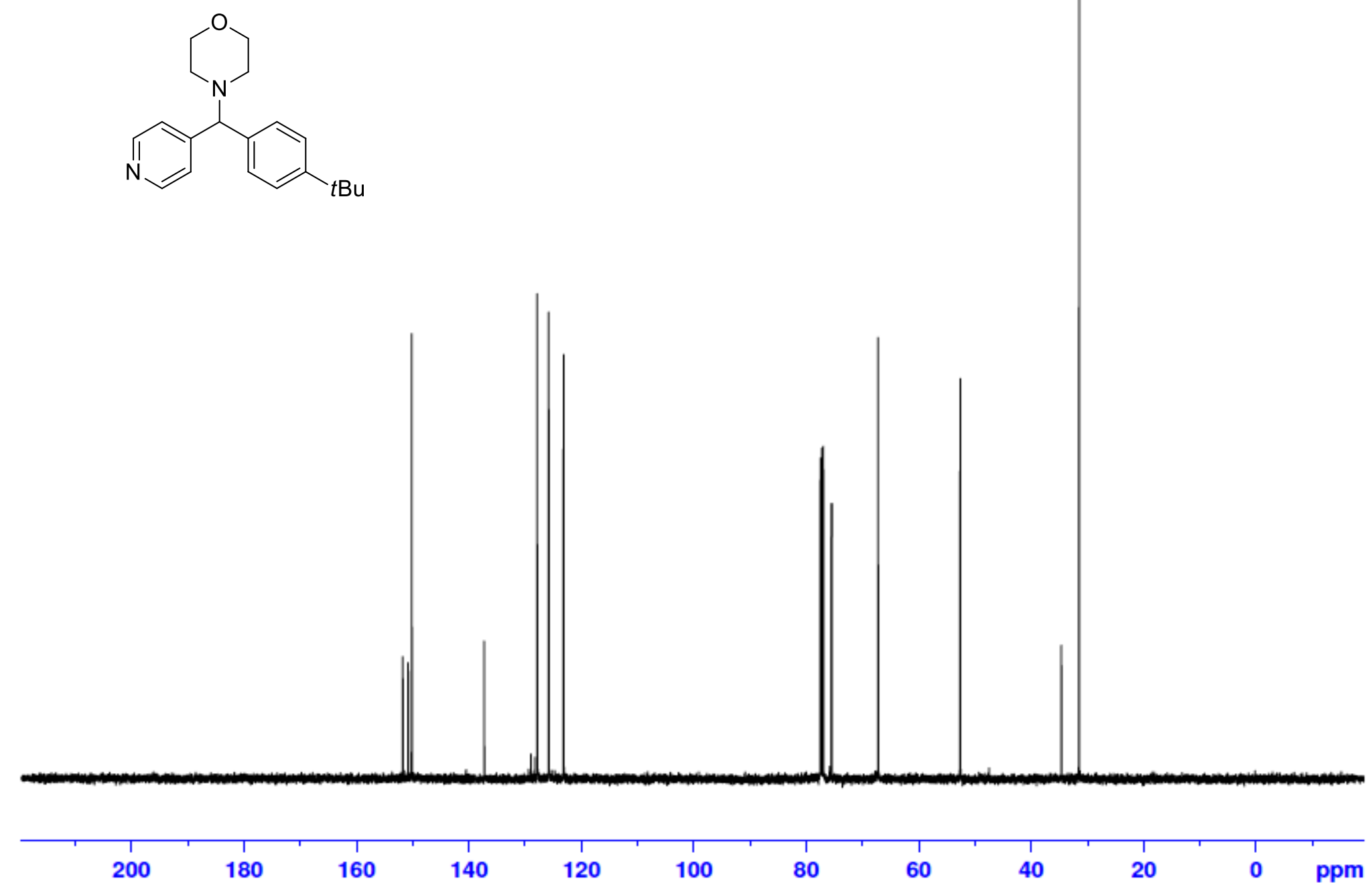
${ }^{1} \mathrm{H}$ NMR (500 MHz) spectrum of $\mathbf{5 d}$.
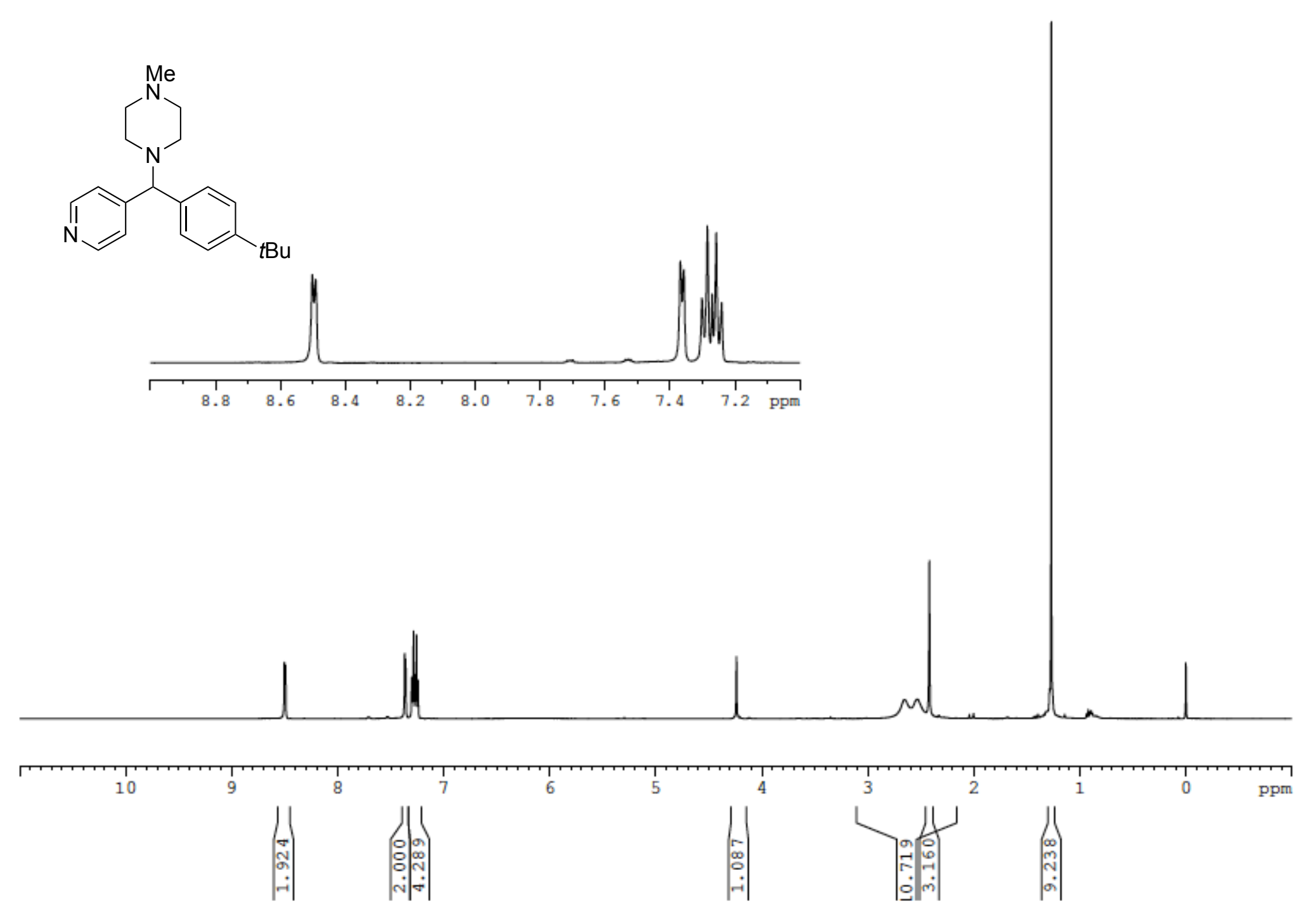
${ }^{13} \mathrm{C}$ NMR (125 MHz) spectrum of $\mathbf{5 d}$.
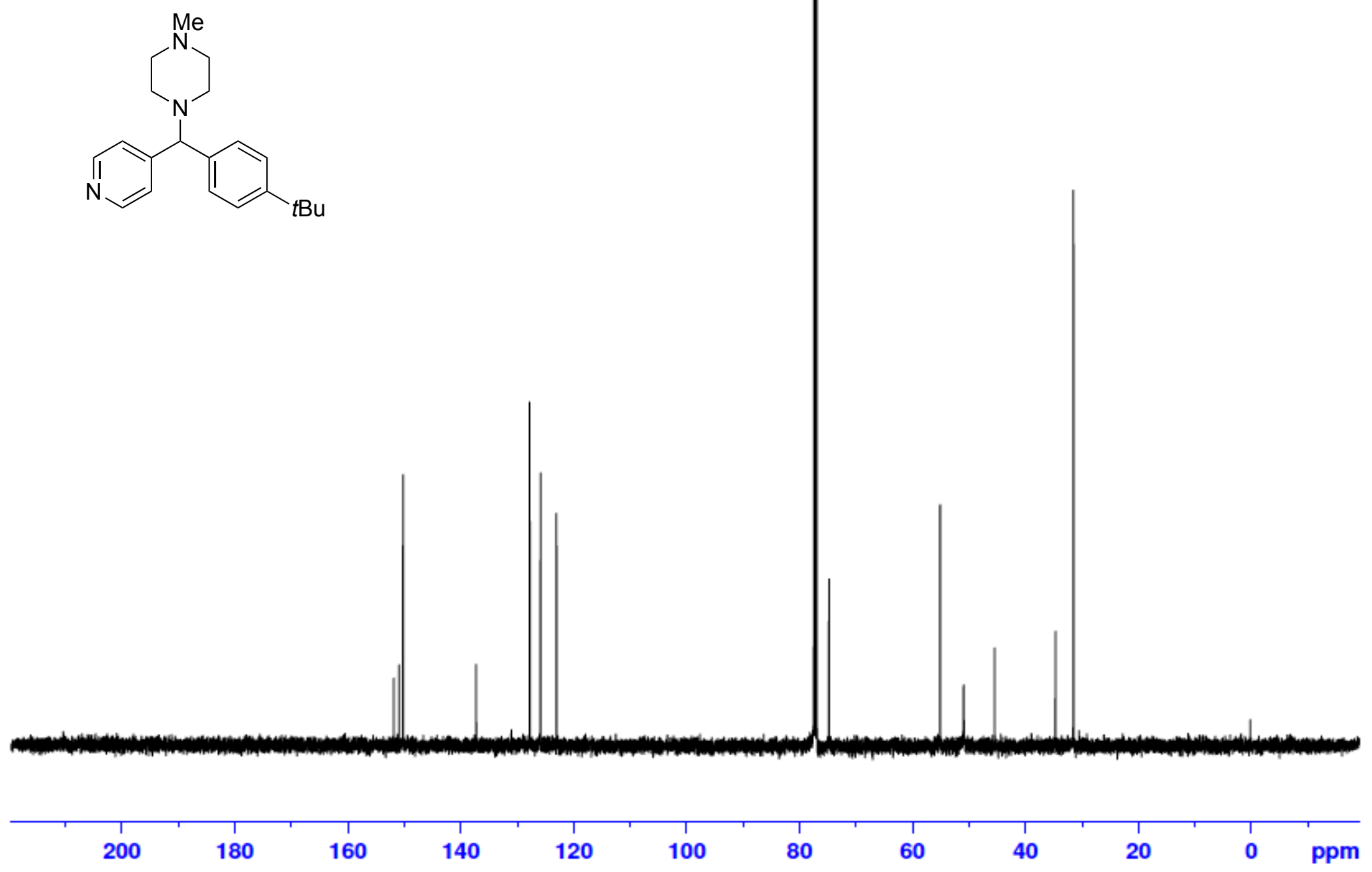
${ }^{1} \mathrm{H}$ NMR (500 MHz) spectrum of $\mathbf{5 e .}$
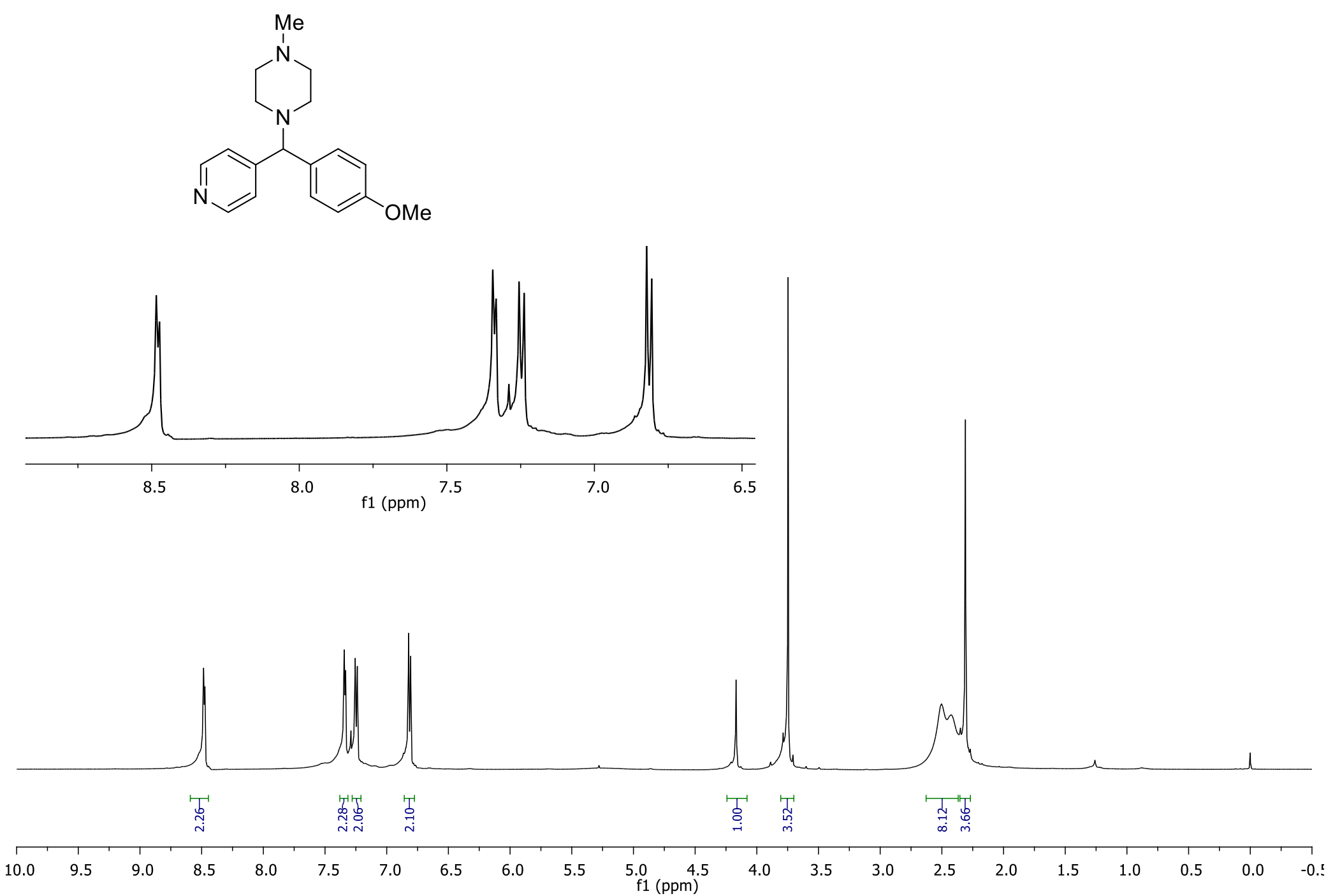
${ }^{13} \mathrm{C}$ NMR (125 MHz) spectrum of $\mathbf{5 e}$.

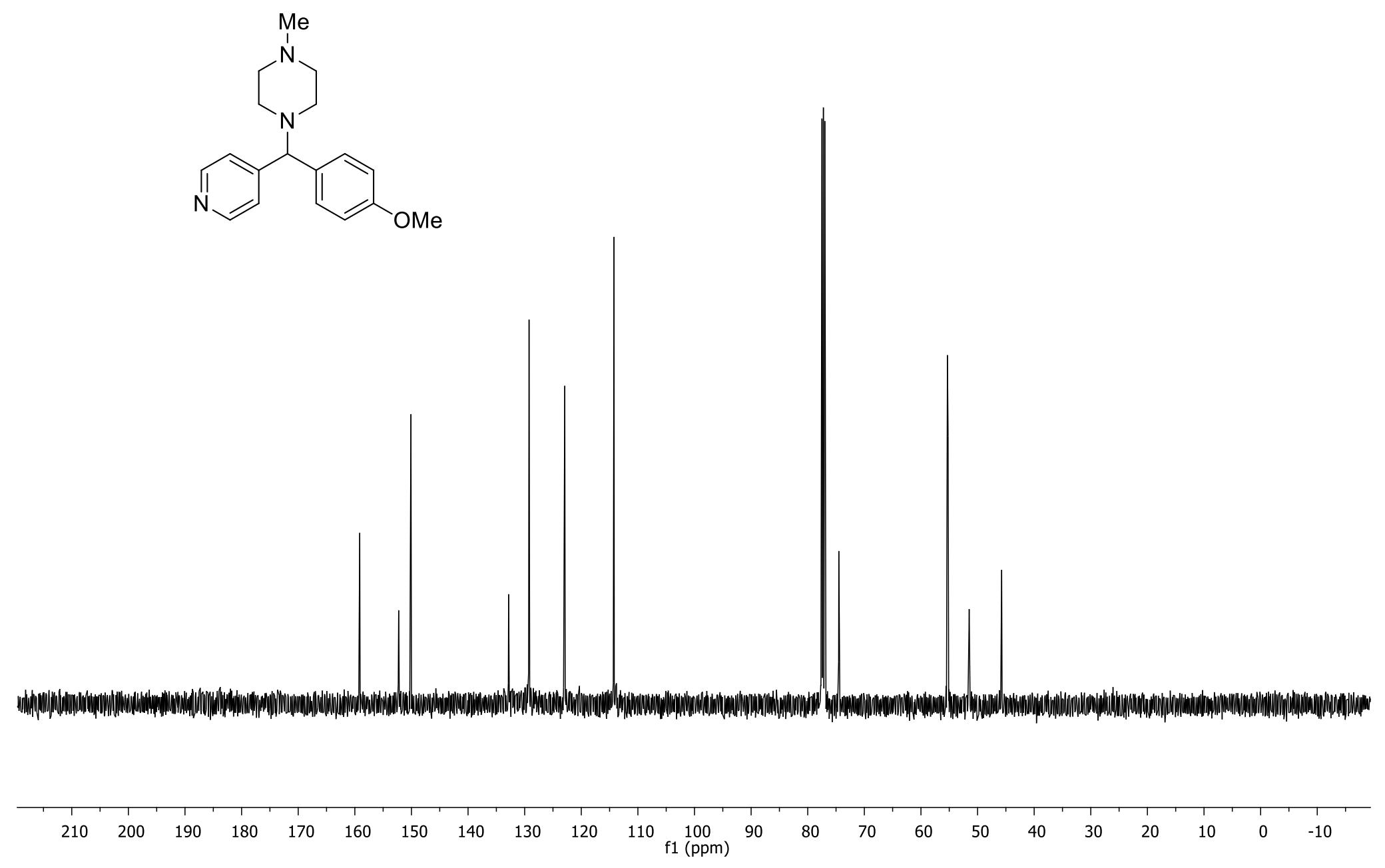


${ }^{1} \mathrm{H}$ NMR (500 MHz) spectrum of $\mathbf{5 f}$.

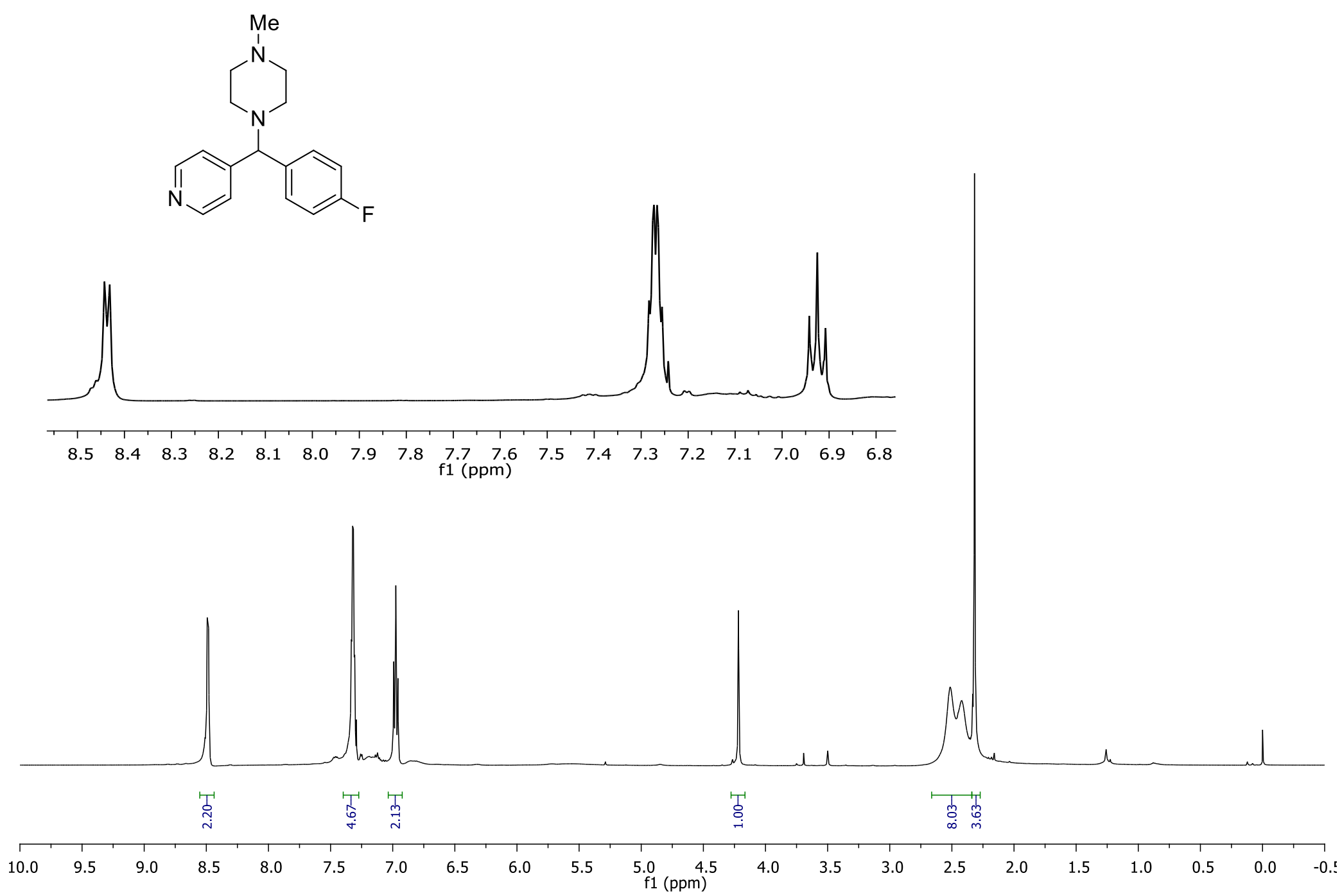




$$
\underline{\underline{I}}
$$


${ }^{1} \mathrm{H}$ NMR (500 MHz) spectrum of $\mathbf{5 g}$.<smiles>CN(C)C(c1ccccc1)c1ccncc1</smiles>
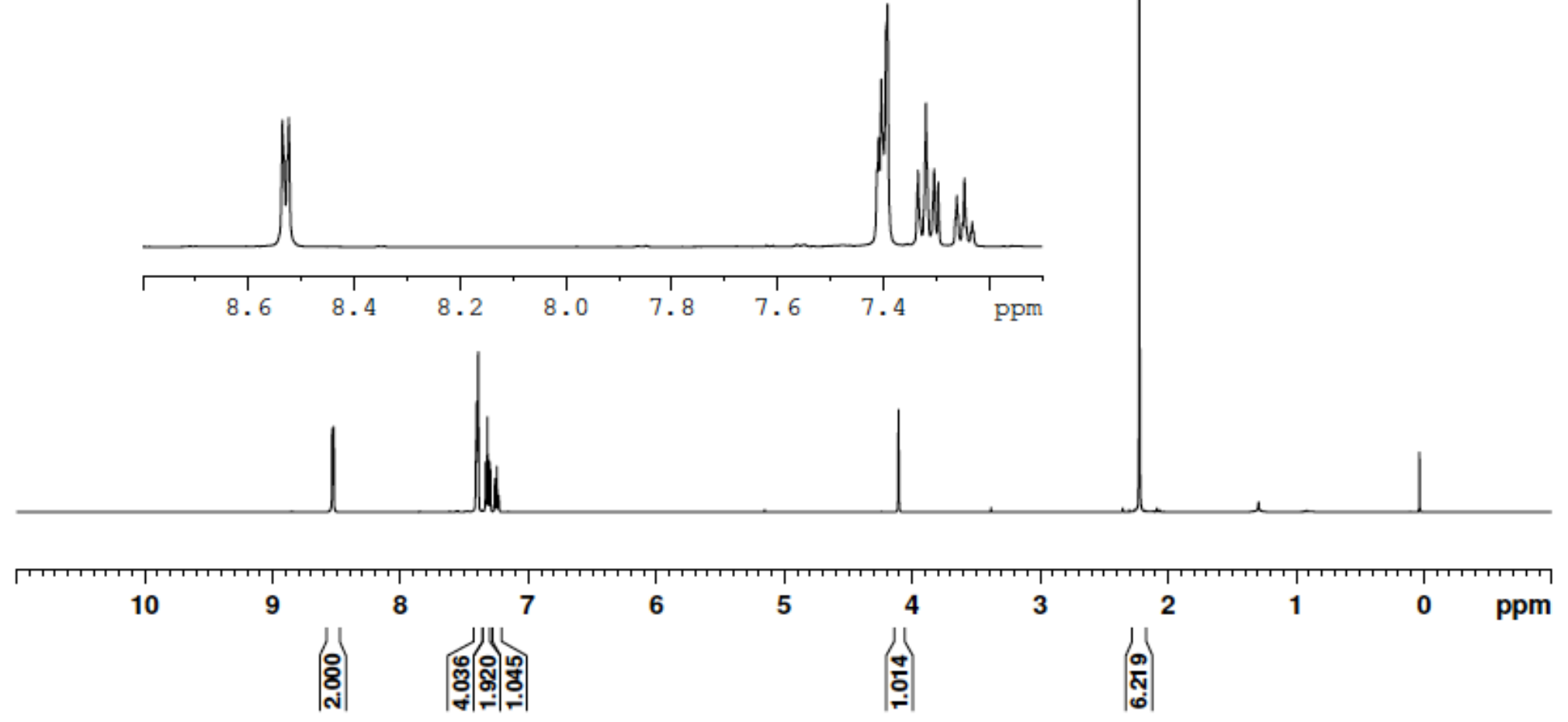
${ }^{13} \mathrm{C}$ NMR (125 MHz) spectrum of $\mathbf{5 g}$.

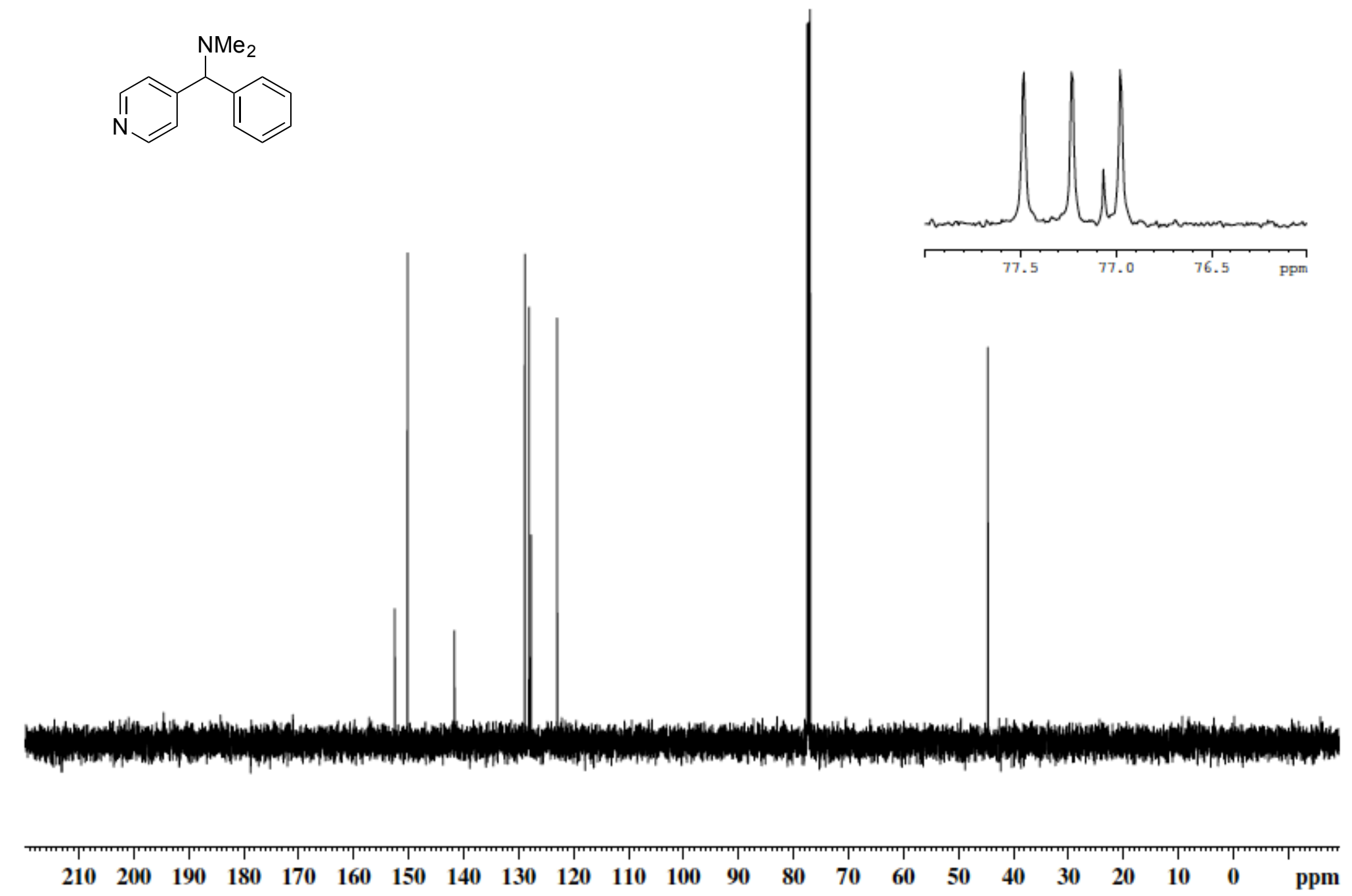


${ }^{1} \mathrm{H}$ NMR (500 MHz) spectrum of $\mathbf{5} \mathbf{h}$.
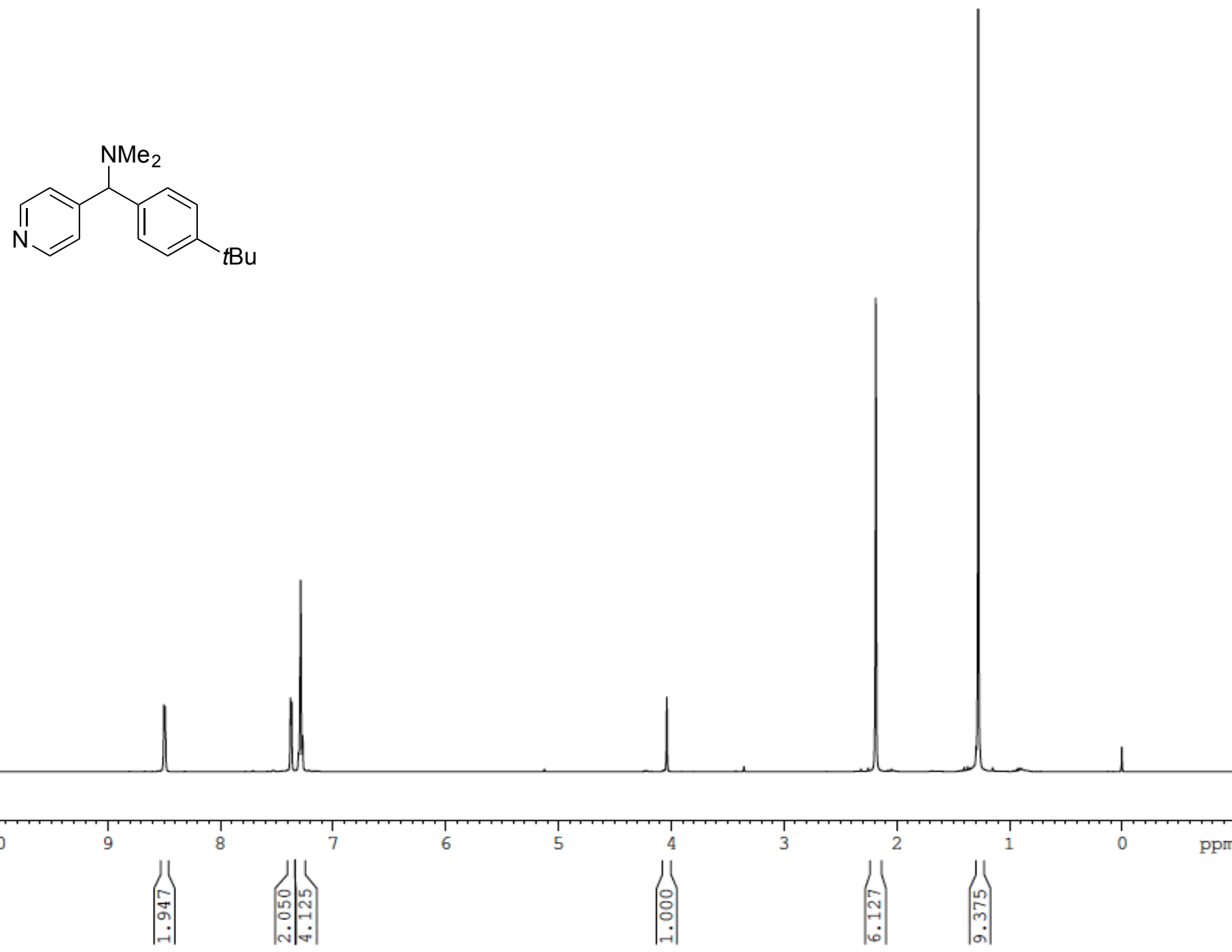
${ }^{13} \mathrm{C}$ NMR (125 MHz) spectrum of $\mathbf{5 h}$.
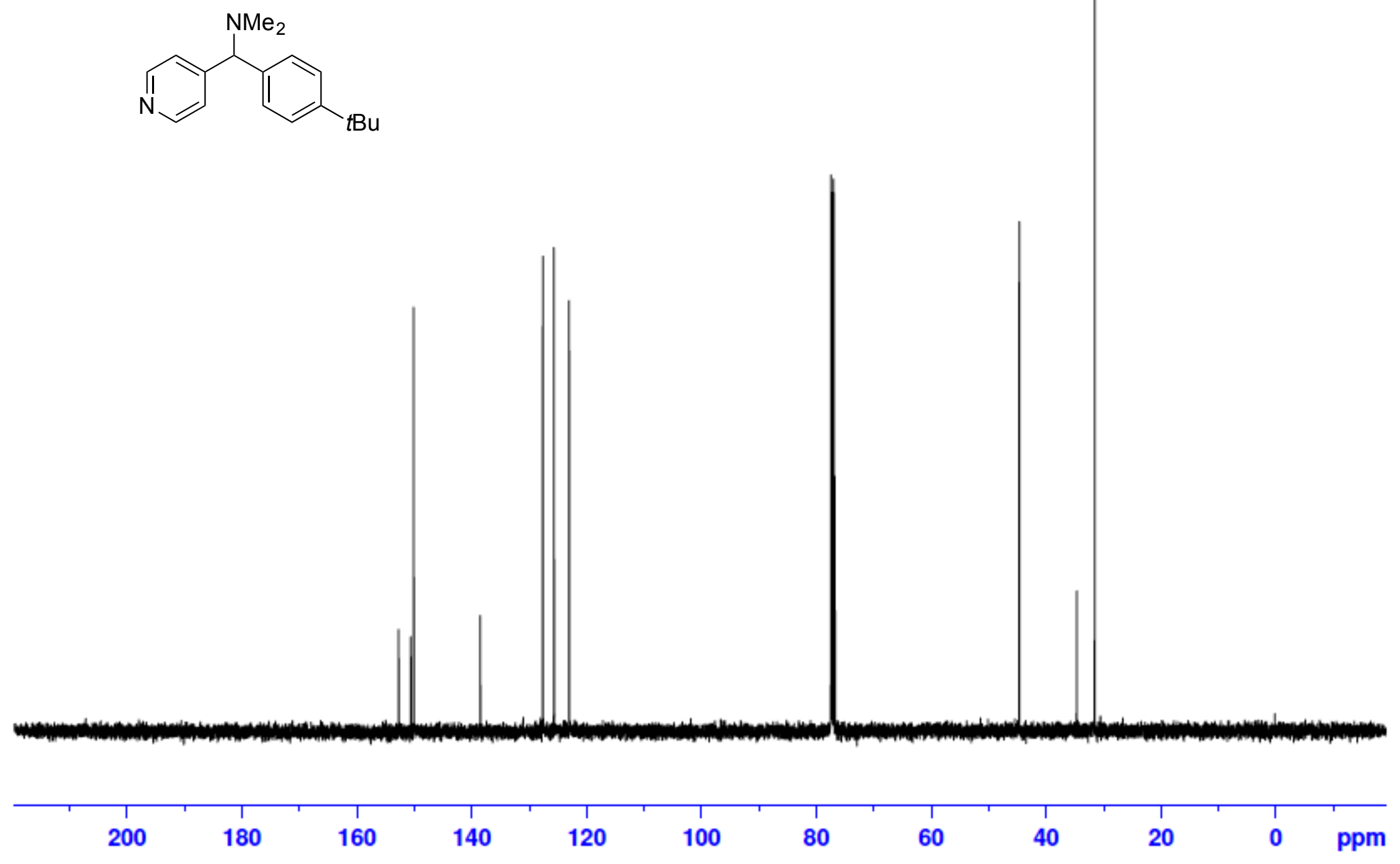
${ }^{1} \mathrm{H}$ NMR (500 MHz) spectrum of $\mathbf{5 i}$.<smiles>COc1ccc(C(c2ccc3ccccc3n2)N(C)C)cc1</smiles>
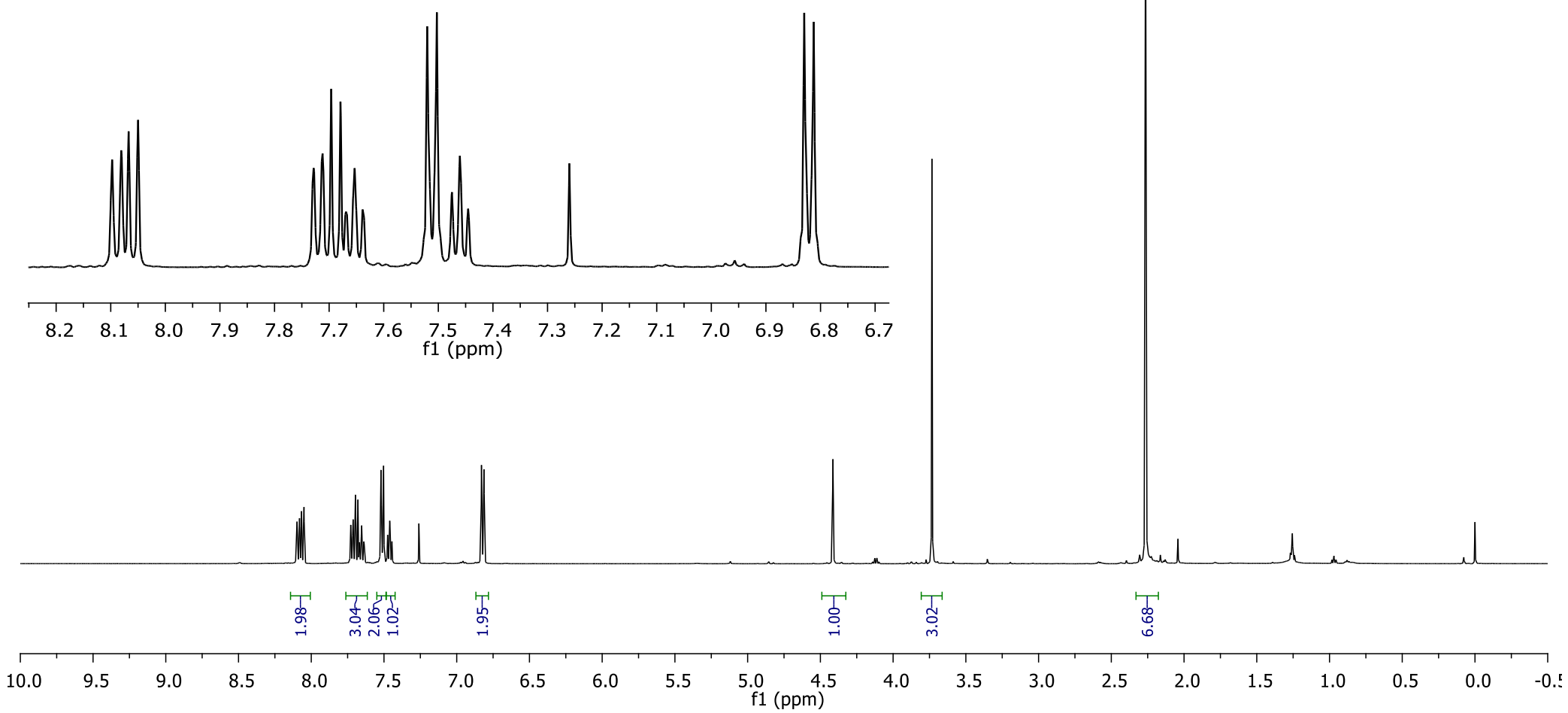

S 125 
${ }^{13} \mathrm{C}$ NMR (125 MHz) spectrum of $\mathbf{5 i}$.

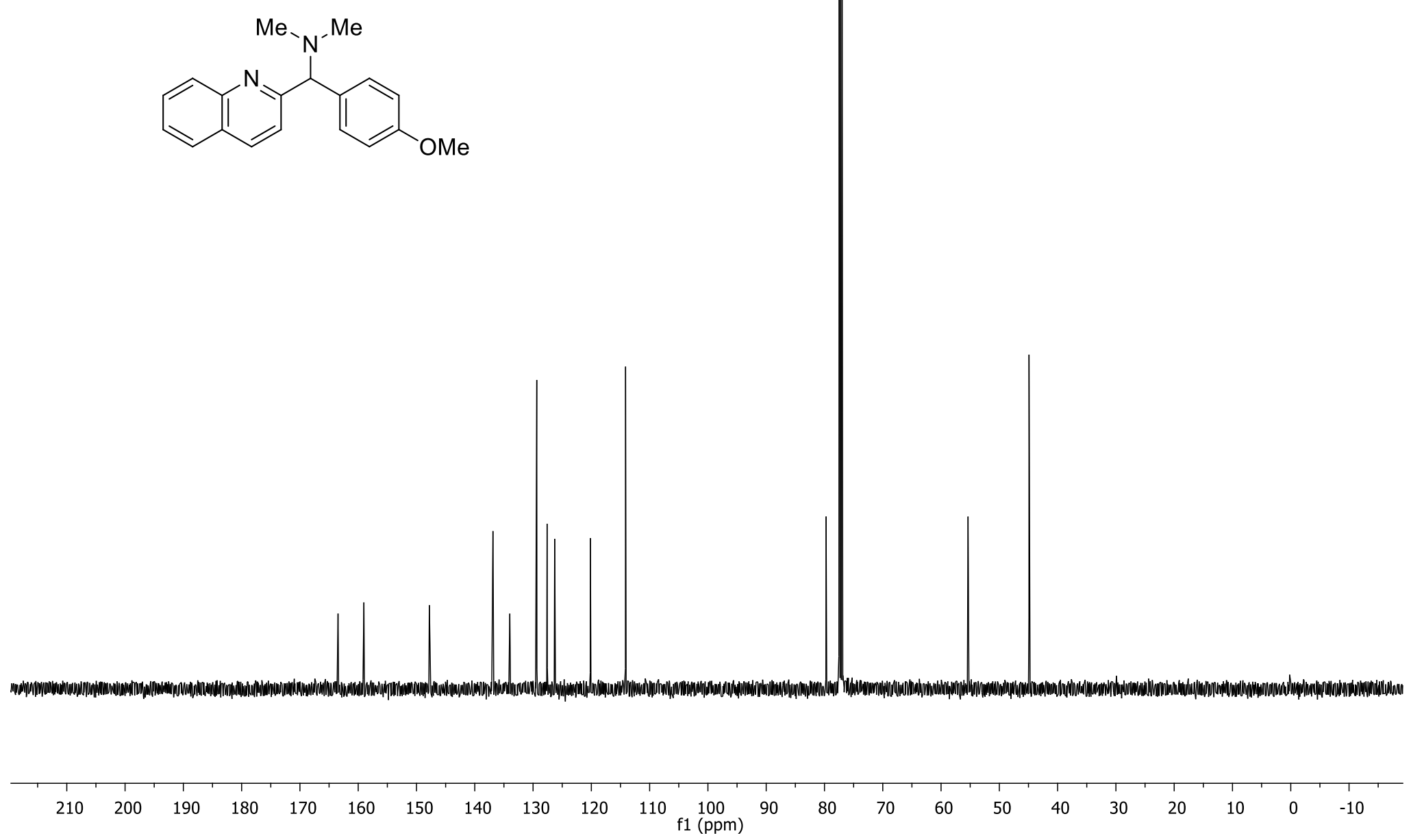

S126 
${ }^{1} \mathrm{H}$ NMR (500 MHz) spectrum of $\mathbf{5 j}$.

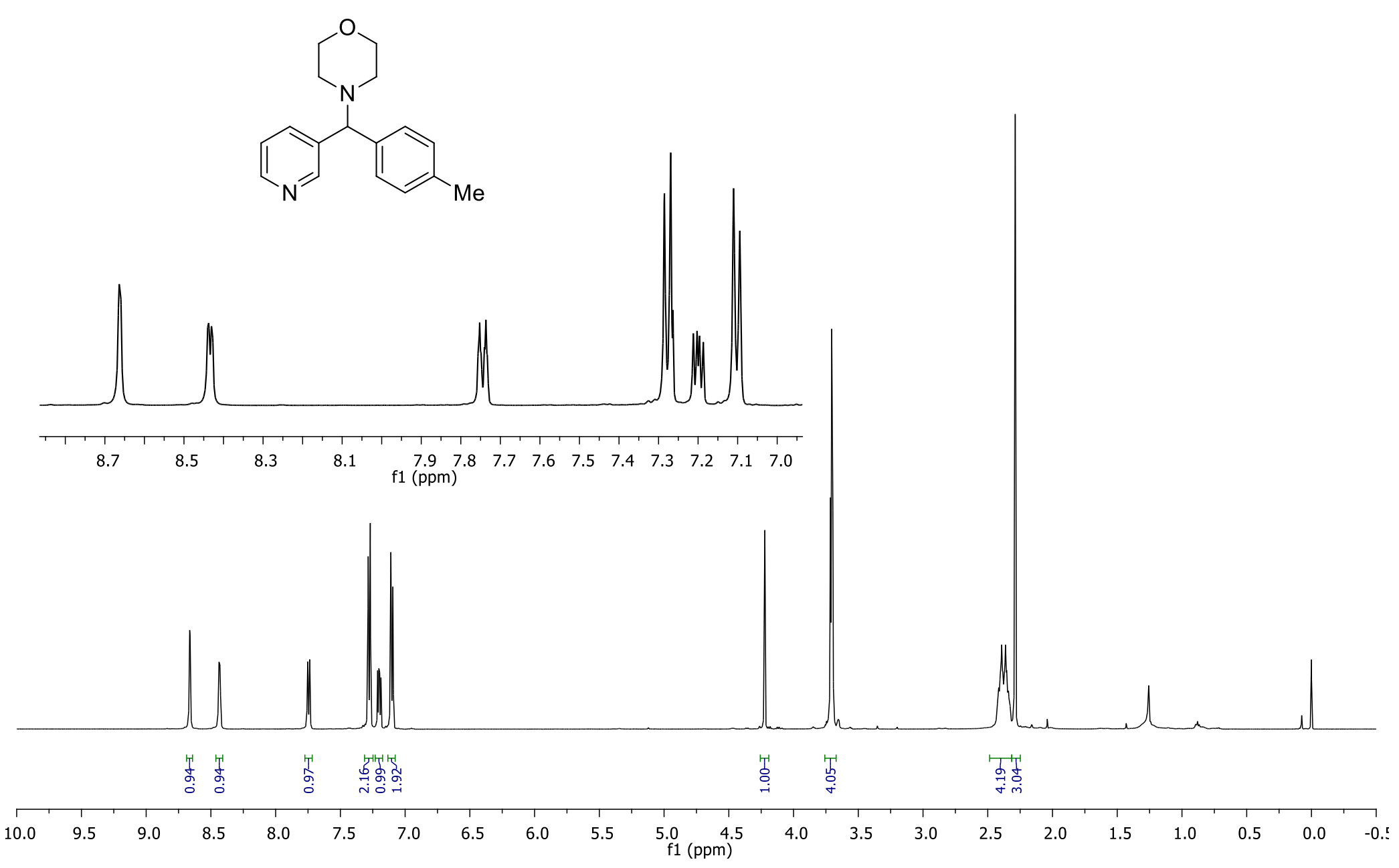


${ }^{13} \mathrm{C}$ NMR (125 MHz) spectrum of $\mathbf{5 j}$.
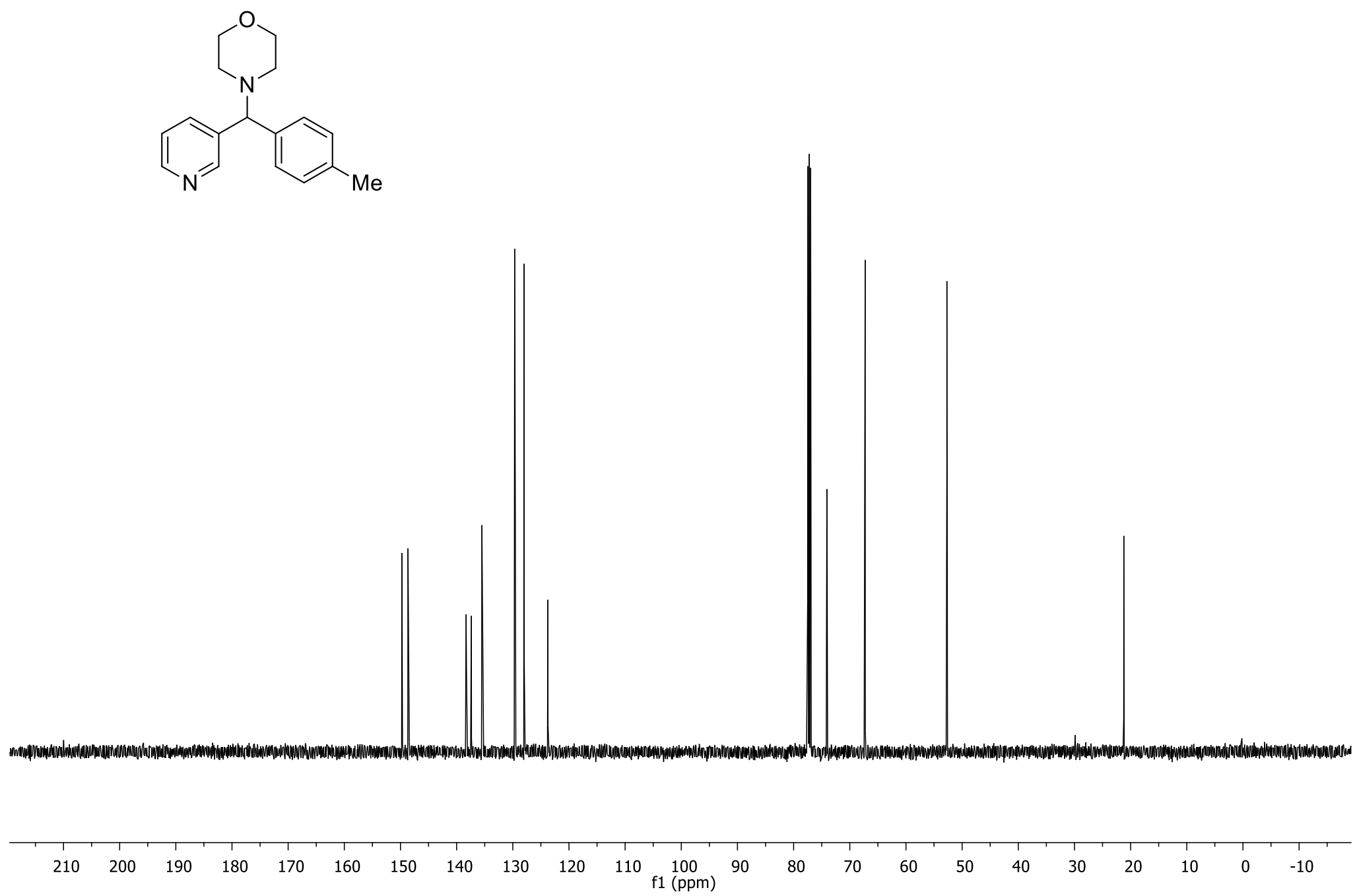

S 128 